\title{
Millimetres do count : methodological and clinical investigations of intra-cranial stereotactic radiotherapy
}

Citation for published version (APA):

Baumert, B. G. (2004). Millimetres do count : methodological and clinical investigations of intra-cranial stereotactic radiotherapy. [Doctoral Thesis, Maastricht University]. Universiteit Maastricht. https://doi.org/10.26481/dis.20041202bb

Document status and date:

Published: 01/01/2004

DOI:

10.26481/dis.20041202bb

Document Version:

Publisher's PDF, also known as Version of record

Please check the document version of this publication:

- A submitted manuscript is the version of the article upon submission and before peer-review. There can be important differences between the submitted version and the official published version of record.

People interested in the research are advised to contact the author for the final version of the publication, or visit the DOI to the publisher's website.

- The final author version and the galley proof are versions of the publication after peer review.

- The final published version features the final layout of the paper including the volume, issue and page numbers.

Link to publication

\footnotetext{
General rights rights.

- You may freely distribute the URL identifying the publication in the public portal. please follow below link for the End User Agreement:

www.umlib.nl/taverne-license

Take down policy

If you believe that this document breaches copyright please contact us at:

repository@maastrichtuniversity.nl

providing details and we will investigate your claim.
}

Copyright and moral rights for the publications made accessible in the public portal are retained by the authors and/or other copyright owners and it is a condition of accessing publications that users recognise and abide by the legal requirements associated with these

- Users may download and print one copy of any publication from the public portal for the purpose of private study or research.

- You may not further distribute the material or use it for any profit-making activity or commercial gain

If the publication is distributed under the terms of Article $25 \mathrm{fa}$ of the Dutch Copyright Act, indicated by the "Taverne" license above, 
MILLIMETRES DO COUNT 
ISBN 9052784345

Copyright $\odot 2004$ Brigitta Baumert, Maastricht

Layout en druk: Datawyse | Universitaire Pers Maastricht 


\section{MILLIMETRES DO COUNT}

Methodological and clinical investigations of intra-cranial stereotactic radiotherapy

\section{ELKE MILLIMETER TELT}

Methodologische en klinische onderzoeken

van intracraniële stereotactische radiotherapie

\section{PROEFSCHRIFT}

ter verkrijging van de graad van doctor

aan de Universiteit Maastricht, op gezag van de Rector Magnificus, Prof. mr. G.P.M.F. Mols, volgens het besluit van het College van Decanen, in het openbaar te verdedigen op donderdag 2 december 2004 om 14.00 uur

door

Brigitta Baumert

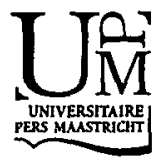


PROMOTOR

Prof. dr. Ph. Lambin

\section{BEOORDELINGSCOMMISSIE}

Prof. dr. E.A.M. Beuls (voorzitter)

Dr. B.J. Heijmen, Erasmus MC Universiteit Rotterdam

Prof. dr. B.J. Slotman, Vrije Universiteit Amsterdam

Prof. dr. J.T. Wilmink

The investigations leading to this thesis have been carried out at the Department of Radiation Oncology, University Hospital Zurich, Switzerland and at the Department of Radiation Oncology (MAASTRO), Academic Hospital Maastricht (azM), Netherlands. Support by the Cancer League, Canton of Zurich, Switzerland and BrainLAB, Munchen, Germany is gratefully acknowledged. Schering Plough, Netherlands and BrainLAB, Germany contributed to the costs of publication of the thesis. 
NICHTS IST

- sagt der Weise.

Du lässt es erstehen.

Es uird mit dem Wind

Deines Atems verwehen

Unmerklich und leise.

Nichts ist. Sagt der Weise.

Mascha Kaleko (1907- 1975)

Für meine Mutter 


\section{Contents}

Chapter 1 GENERAL INTRODUCTION 9

Definition of conformality 10

Basis of conformality 13

Three-dimensional irradiation techniques $\quad 15$

Sparing of normal tissue $\quad 18$

Clinical outcome 19

Objectives of the thesis 20

PART I: ASSESSMENT OF SET-UP ACCURACY

Chapter 2 Repositioning accuracy of fractionated stereotactic irradiation: Assessment of isocentre alignment for different dental fixations by using sequential CT scanning

PART II: METHODOLOGICAL INVESTIGATIONS

Chapter 3 Intensity modulated stereotactic radiotherapy versus stereotactic conformal radiotherapy for the treatment of meningioma predominantly located in the skull base

Chapter 4 A comparison of dose distributions of proton and photon beams in stereotactic conformal radiotherapy of brain lesions 67

Chapter 5 Dose conformation of intensity modulated stereotactic photon beams, proton beams and intensity modulated proton beams for intracranial lesions

\section{PART III: CLINICAL OUTCOME}

Chapter 6 Fractionated stereotactic radiotherapy boost after postoperative radiotherapy in patients with high grade gliomas 
Chapter 7 Early improvements in vision after fractionated stereotactic radiotherapy for primary optic nerve sheath meningioma

Chapter 8 Stereotactically guided conformal radiotherapy for progressive low grade gliomas of childhood

Chapter 9 GENERAL DISCUSSION AND SUMMARY

Introduction 160

Accuracy 161

Evaluation of conformality and different 3D-CRT techniques 168

Clinical Outcome $\quad 162$

Conclusions 165

Future perspectives $\quad 166$

$\begin{array}{ll}\text { SAMENVATTING } & 177\end{array}$

$\begin{array}{ll}\text { Curriculum vitae } & 181\end{array}$

List of publications 182

$\begin{array}{ll}\text { Acknowledgement } & 184\end{array}$ 
CHAPTER 1

General introduction 
Cancer increasingly contributes to morbidity and mortality worldwide. It is the second leading cause of death (with ca. 25\%) in the U.S., where the crude incidence rate is $>300$ new cases per 100.000 inhabitants per year ${ }^{60}$. The highest incidence rates are prostate cancer in men and breast cancer in women. Lung cancer is the second leading cancer type in both men and women. The same numbers also apply for Europe ${ }^{4,16}$. Tumours of the central nervous system (CNS) are less common. The overall incidence of primary brain tumours is around 7 per 100,000 population in the Netherlands and in Europe (Netherlands Cancer Registry 200175). Although brain tumours account for less than $2 \%$ of all primary cancers, they probably represent the greatest variety of tumours of any site in the body ${ }^{36}$. The variety in brain tumours necessitates a close tailoring of the treatment to the disease in order to optimise outcome. Whilst some brain tumours are highly curable (for example meningioma), many are extremely lethal as for example high-grade astrocytoma.

Radiotherapy is one of the main treatment modalities for cancer. About $50 \%$ of patients will receive radiotherapy during the management of their disease. It is, like surgery, a loco-regional treatment modality and therefore has its place in the management of brain tumours. Local control is critical in brain tumours, as it is directly related to survival and there is usually no risk of haematogenic and lymphogenic spread. Pathologically benign tumours such as meningioma can also be lethal because of the enclosed nature of the brain and its lack of tolerance to damage mainly due to increased pressure within the cranium. Nevertheless, a considerable group of patients with benign neoplasms (e.g. intra-cranial germ cell tumours, meningiomas) can be cured. Especially for the latter, there is a need to improve survival and local control rates and to perceive methods of reducing toxicity. This is the aim of conformal radiotherapy.

\section{DEFINITION OF CONFORMALITY}

Over the last decades important advances have been made in radiation therapy. Conformal radiotherapy has evolved from the development of sophisticated treatment planning systems, made possible by the availability of fast computers with a large storage capacity, as well as from technical and mechanical developments in treatment delivery. The term "conformal radiotherapy" covers a wide spectrum of radiotherapy treatment approaches from adding simple blocking devices to a field for shielding normal tissue to the sophisticated techniques employed in intensity modulated radiotherapy (IMRT). In general, conformal radiotherapy is known as three-dimensional conformal radiotherapy (3D-CRT), based on three-dimensional (3D) patient data, treatment planning and treatment delivery. Stereotactic radiotherapy and proton beams with or without intensity modulation represent the more 


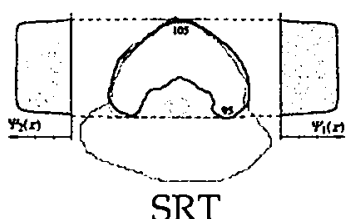

STANDARD RADIATION THERAPY $1930-\quad P_{+} \approx 40-50 \%$

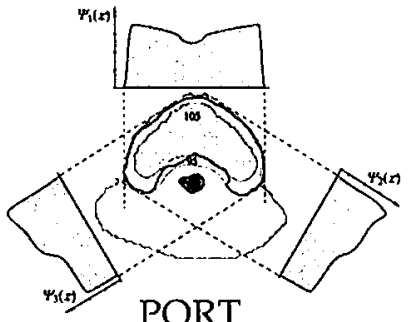

PORT

Physically Optimized RADIATION THERAPY 1985- $\quad P_{+} \approx 55-75 \%$

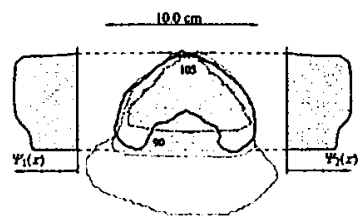

IMRT

INTENSITY MODULATED RADIATION THERAPY 1960- $P_{+} \approx 50-65 \%$

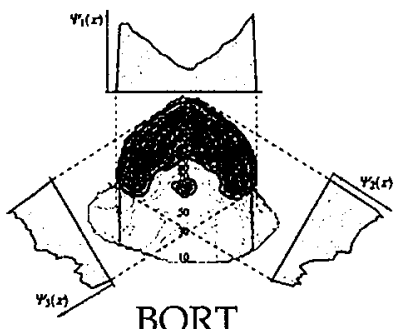

Biologically OPTIMIZED RADIATION THERAPY 1990- $P_{+} \approx 60-85 \%$

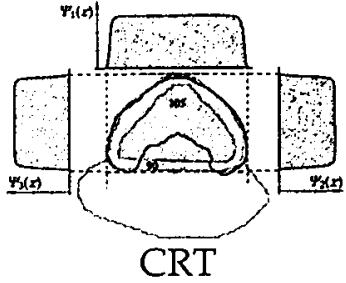

CONFORMAL Radiation THERAPY 1965- $P_{+} \approx 45-60 \%$

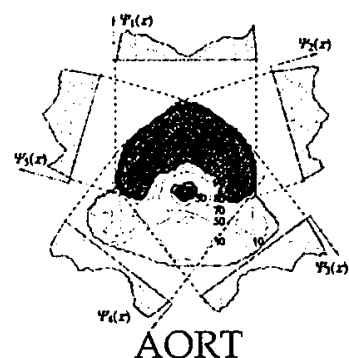

Predictive Assay Based BIOLOGICALLY OPTIMIZED RADIATION THERAPY 2000- $P_{+} \approx 65-90 \%$

Figure 1. Steps in the development of conformal radiation therapy techniques. Approximate year of introduction and the resultant complication-free cure, $\mathrm{P}_{+}$, compared with that of standard radiation therapy, are also indicated. It is seen that the commonly used term conformal therapy does not necessarily imply a very large improvement in $P_{+}$. The lower row of panels in principle are special cases of IMRT and CRT but in general such high degrees of conformity cannot usually be obtained only by absorbers or dynamic collimation.

(Reprinted from International Journal of Radiation Oncology Biology Physics, 49(2), Brahme A.: "Individualizing cancer treatment: biological optimization models in treatment planning and delivery", pp 327-337, Copyright 2001, with permission from Elsevier)

sophisticated conformation techniques of 3D-CRT. Beside the physically optimised treatment techniques using CRT and IMRT, biological optimisation using for example predictive assays of radiation sensitivity are evolving ${ }^{7}$ (Figure 1 ).

For this work, we define the aim of using 3D-CRT as: the use of techniques in which the surface of a reference isodose volume is adjusted to match the surface of the target volume ${ }^{18}$. As a consequence, radiotherapy beams are adjusted (tailored) such that the volume receiving at least $95 \%$ of the prescribed dose conforms closely to the shape of the target while sparing surrounding normal tissue ${ }^{11}$. The level of conformity achieved in a treatment set-up can be quantified by a conformity index (Figure 2). It can be reasonably assumed that a high level of dose conformity will improve the efficacy of treatment by decreasing normal tissue toxicity and thus allowing a tumour dose escalation and therefore increased tumour control. For example, if conventional radiotherapy 

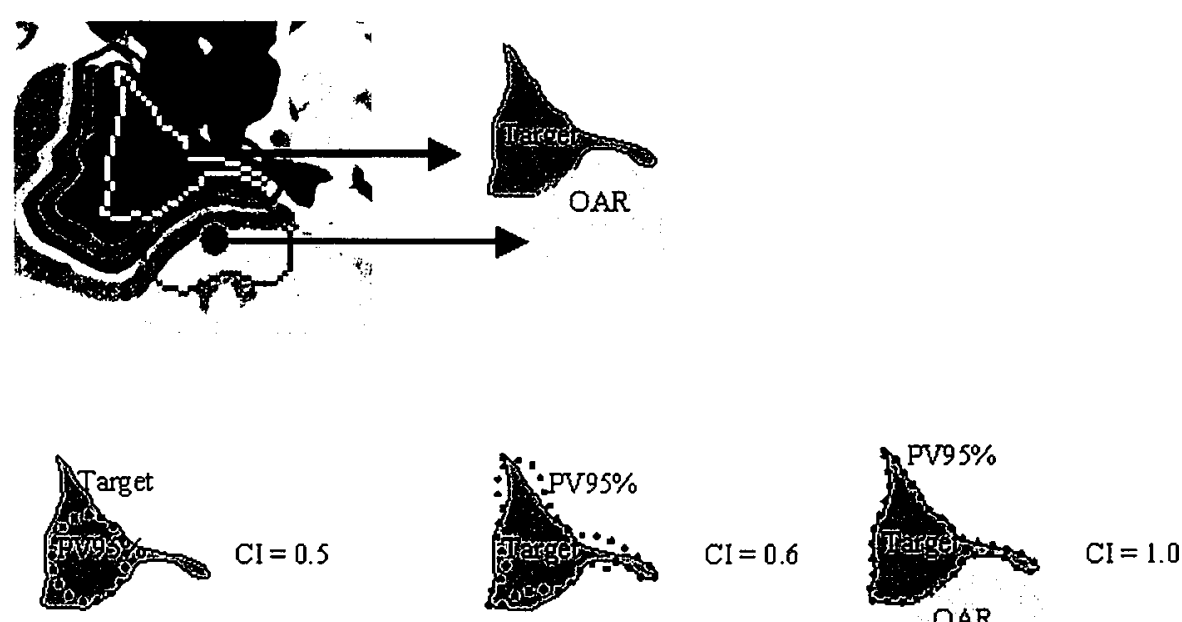

OAR

Figure 2. Definition of conformity index of the $95 \%$ isodose (based on: Paddick I, $J$ Neurosurg $2000^{53}$ ). Schematic drawings of different isodose conformalities with its conformity index based on a skull base meningioma with an organ-at-risk (OAR) nearby (here the brainstem). A conformity index is a measure of how accurately the prescription isodose volume (the volume of the $95 \%$ ) conforms around the target volume. A value of 1.0 would be an ideal conformation of the $95 \%$ isodose (PV95\% = prescription isodose volume of the $95 \%$ isodose volume) around the target volume where also the OAR would be best spared.

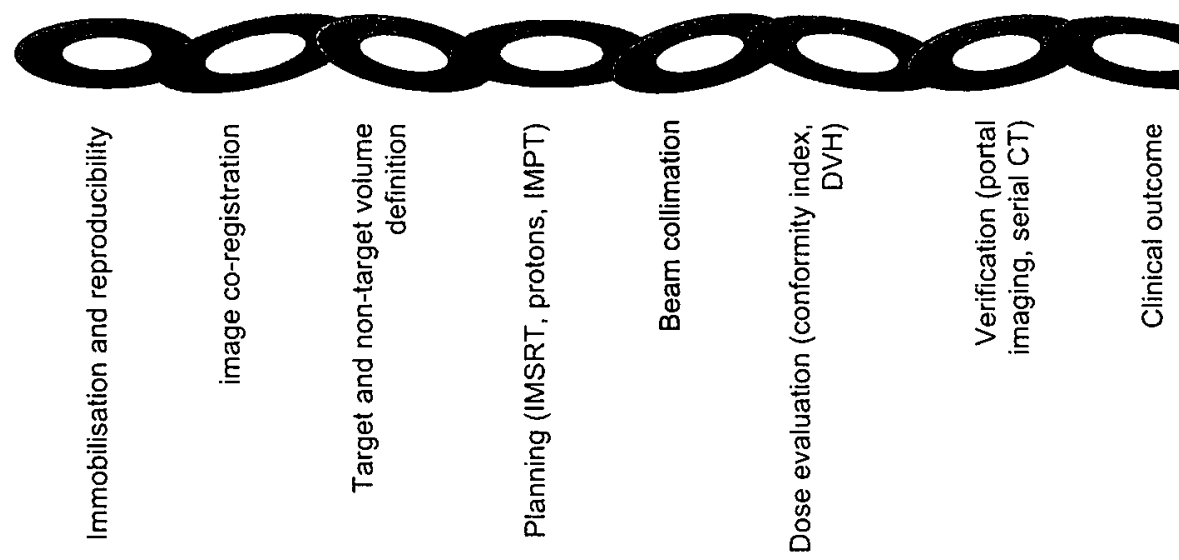

Precision 3D Planning

Figure 3. Chain of conformal radiotherapy processes (based on Webb St, 19937"). 
planning (based on manually transferred tumour volume data from diagnostic computer tomography (CT) images) is compared with CT-based fully 3D planning with the use of beam's-eye-views (BEV) in patients with a primary brain tumour (astrocytoma), this results in a 30\% reduction in the volume of normal brain tissue treated to a high-dose level ( $>95 \%$ isodose line $)^{70}$. Furthermore, a $50 \%$ reduction of normal brain irradiated is observed ${ }^{70}$.

\section{BASIS OF CONFORMALITY}

\section{Accuracy: Reproducibility}

There are many steps leading to the delivery of 3D-CRT. The main ones are data acquisition, 3D treatment planning and optimisation, 3D dose delivery, and treatment verification $932,38,59$. Treatment preparation and delivery of 3D-CRT can be compared with the construction of a chain ${ }^{79}$ : all links must be sound and properly constructed because the overall strength of the chain is limited by that of its weakest link. Figure 3 summarizes the main links of 3D-CRT chain and its relation to this thesis. Stereotactic radiotherapy is a highly accurate form of 3D-CRT and therefore the same conditions apply.

Tools like BEV, dose-volume histograms (DVH), 3D dose calculations and computer-driven multi-leaf collimators (MLC) are the basis of 3D-CRT. The first step, data acquisition, incorporates patient immobilization, imaging and image co-registration. The accuracy of patient and treatment unit (re)positioning defines the tumour to target volume margin (safety margin) to be added for $3 \mathrm{D}$ planning.

Accuracy is the mainstay in the treatment of brain tumours using stereotactic conformal radiotherapy (SCRT). Accuracy is necessary in order to reduce safety margins around the tumour, which in turn reduces the volume of normal tissue receiving a high dose of radiation and thus the incidence and gravity of side effects. SCRT combines the precision of the stereotactically guided tumour localisation to the radiobiological advantages of fractionation. This implies the necessity for a highly accurate repeated alignment of the planned isocentre with the isocentre of the linear accelerator. For fractionated SCR T a non-invasive immobilization system is used. A relocatable frame or mask system is often combined with a dental fixation in order to increase reproducibility and the accuracy of daily set-up. Published data indicate a set-up accuracy of relocatable systems of $\leq 2 \mathrm{~mm}^{1,21,24} 27,33,34,39,50,61,62,81$. 


\section{Accuracy: Target volume definition}

The tighter the margin, the smaller the volume to be irradiated. This, however, postulates a highly accurate definition of the tumour itself. The exact target definition is crucial in order to reduce the risk of a geographical miss. There is some concern that this could have an adverse effect on efficacy of treatment, defined as local tumour control.

Within the brain, exact tumour definition (GTV) and clinical target volume (CTV) delineation seem to be more difficult than for many other organs due to insufficient knowledge on possible infiltration outside of the visible tumour boundaries ${ }^{44}$. Recently, S. Bentzen ${ }^{3}$ proposed a new concept of a "real target volume" (RTV). This would be defined as the tissue volume that would require irradiation to control loco-regional disease and it could be larger or smaller than the CTV and one may not necessarily be a subset of the other ${ }^{3}$. This speculative concept is based on upcoming definitions of tumour spread by for example pathologic assays or molecular profiling. Also, new imaging techniques such as functional imaging with magnetic resonance spectroscopy (MRS) or positron emission tomography (PET) seem to provide a solution. For example, low-grade glioma are best imaged on T2 weighted MR images. However, despite using advanced imaging techniques, target volume delineation in low-grade glioma remains difficult. Metabolic imaging with MRS measuring metabolic tumour activity reveals that cellular infiltration of low-grade glioma tumour cells is mainly restricted to the T2 weighted hyper-intensity area on MRI ${ }^{57}$. Similar observations are reported by Murphy et al. ${ }^{51}$ who monitored treatment response of low-grade glioma with proton MRS. The use of co-registered MRS to the planning CT could therefore reduce the CTV.

Another functional imaging method is PET, where the choice of the best tracer is of importance. Amino acid tracers as 11-C-methionine for PET are more sensitive in differentiating between tumour and normal brain than $18-$ FDG $^{29,72}$, because their background uptake in normal brain tissue is low, thus providing good contrast for the tumour itself. These tracers may therefore be useful in treatment planning: first studies have shown a possible reduction of radiotherapy treatment volumes by the use of PET or possibly a better definition of boost volumes ${ }^{71,26}$. Additionally, if histological measurements are compared with their imaging counterpart an over- or underestimation of the imaging procedure may be observed, where each imaging technique (PET, CT or MRI) shows a different deviation from the surgical specimen. So far this has been shown for pharyngo-laryngeal squamous cell carcinomas only ${ }^{12}$. There is still a lack of validation of imaging by pathology for other cancers. 


\section{Accuracy: Image co-registration}

Another issue is image fusion: not only accuracy of the fusion process itself has to be accounted for when defining the tumour, but also different information of different imaging techniques. Khoo et al. ${ }^{35}$ have shown, that MRI based target volumes of skull base meningiomas are principally larger than the equivalent $\mathrm{CT}$ based volumes. Although the average vector difference was small, the differences in individual borders could be large and CT or MR volumes can vary appreciably, because they provide complementary information ${ }^{35}$.

\section{THREE-DIMENSIONAL IRRADIATION TECHNIQUES}

The aim of three-dimensional conformal radiotherapy (3D-CRT) is to decrease the volume of normal tissue irradiated for the same target volume. This can be achieved by the use of non-coplanar beams shaped by customized shielding blocks, circular cones or multi-leaf collimators or more recently, by intensity modulated beams.

\section{Stereotactic radiotherapy}

The concept of stereotactically guided three-dimensional definition of an intra-cranial lesion as a $3 \mathrm{D}$ target calculation in the brain was first published by Horsley and Clarke in $1908^{30}$. They developed a frame, which was fixed to the skull and which was based on a Cartesian co-ordinate system. This served as immovable reference for localisation of intra-cranial targets. Positional information is transferred from images onto the 3D coordinate system of the stereotactic frame (Figure 4). This system has been adapted for irradiation of intra-cranial lesions and first used by the Leksell Gammaknife ${ }^{\circledR}$ in $1968^{43}$. Using this stereotactically-guided radiotherapy technique, a steep dose gradient between target and normal tissue can be achieved. Stereotactic irradiation can be given as a single high dose fraction, in which case it is termed stereotactic radiosurgery (SRS), because of the analogy to a surgical act of removing the tumour. Stereotactic irradiation can also be given in a fractionated schedule, when it is termed SCRT. Linear accelerator based SRS or SCRT uses sets of multiple intersecting non-coplanar arcs wit circular collimators, which are ideally suited to spherical or ellipsoidal targets.

Over the past years the use of non-coplanar, static fields shaped by micro-multileaf collimators ( $\mathrm{mMLC}$ ) or conformal lead blocking has been introduced. This results in a better dose conformation, particularly for irregularly shaped lesions $s^{6,10,23,28 \text {, }}$ $40,41,47,65$. The use of circular collimator with arc rotation techniques is limited, as, for better conformation, multiple isocentres have to be used, which is quite time con- 


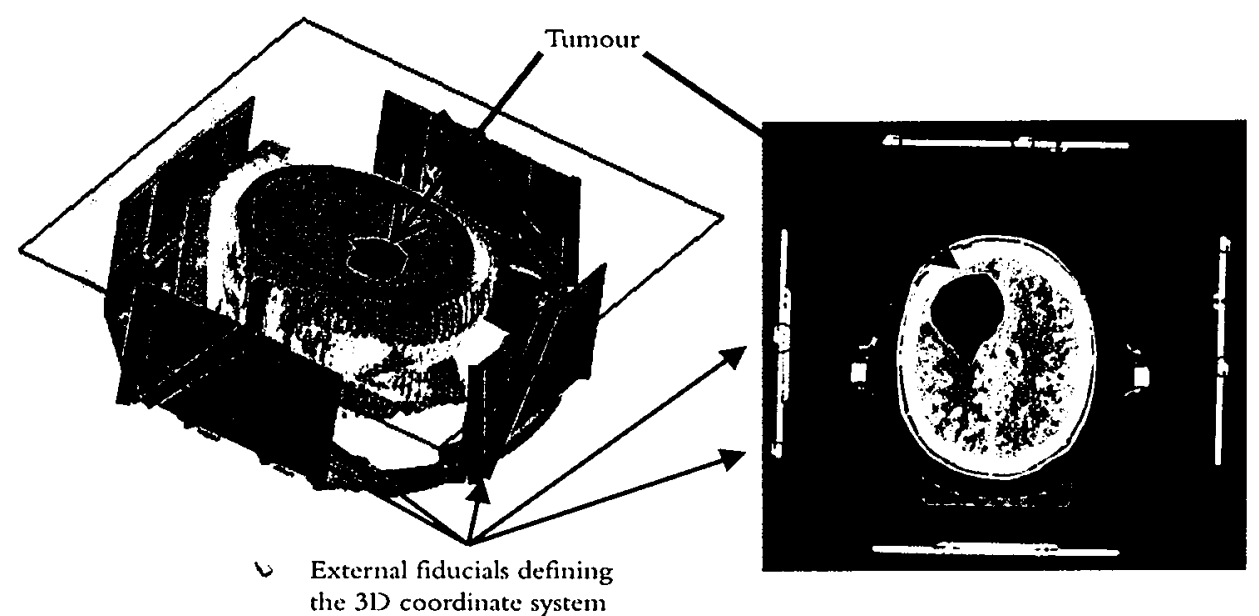

Figure 4. Threc-dimensional reconstruction of a stereotactic planning CT scan with a cranial view of a stereotactic localizer in situ. External fiducials are used to define a stereotactic space within which a point can be localized with the stereotactic co-ordinate system.

suming and often results in a significant dose inhomogeneity within the target volume.

\section{Proton therapy}

High-energy protons are used in radiation therapy because of their specific energy deposition characteristics. As they traverse matter, protons lose their energy mainly through collisions with electrons, which leads to ionisation of the atoms along their paths. Unlike photons where an exponential absorption of energy takes place, the rate of energy loss per unit length of matter traversed by a proton beam is low at first but increases as the protons slow down. Most of the energy is deposited near the end of the range in a region called the Bragg peak (Figure 5). The localisation of dose in the Bragg peak provides an ideal characteristic for dose conformation to target volumes $37,67,80$. The depth of the Bragg peak in a given material is dependent on the beam's energy. The beam's energy can be modulated and thus the Bragg peak can be spread between the distal and proximal ends of a tumour forming a so-called "Spread Out Bragg Peak (SOBP)". In one special treatment delivery technique known as the spot scanning technique as used at the Paul-Scherrer-Institute (PSI) Switzerland, the proton beam can be shaped to deliver a $3 \mathrm{D}$ conformal dose distribution ${ }^{46}$. The advantage of this system is that proton beams can be made to conform to complex target shapes whilst preserving good dose homogeneity within the treatment volume. Apart from excellent conformity afforded by protons, the sharp dose fall off beyond 
the Bragg peak reduces the integral dose to normal tissue for any given treatment modality as there is no exit dose. Proton beams have been used in radiotherapy and radiosurgery for a number of years where these beam characteristics have been put to good use, especially in paediatric tumours, skull base tumours and uveal melanoma $15,48,49,68,74$.

\section{Intensity modulated radiation therapy}

The addition of modulation to the beam intensity is the most recent development in $3 \mathrm{D}-\mathrm{CR} \mathrm{T}$. Intensity modulated radiotherapy uses $3 \mathrm{D}$ treatment planning techniques to optimise delivery of radiation to irregularly shaped volumes ${ }^{73}$. This is reached by a variation or modulation of the beam intensity across the treatment field, which is achieved by dividing one beam into multiple beamlets. The intensity of each beamlet is modified depending on whether tumour or critical normal structures are present in the beam pathway. This is different from traditional 3D-CRT where the beams are shaped to a projection of the PTV by the use of beam's-eye-views from chosen directions (forward planning). For intensity modulated radiotherapy the shaping and intensity variations are determined by an optimisation algorithm, which seeks the best solution to a set of pre-defined dose constraints (inverse planning). Intensity modulation techniques are now available for both photon stereotactic treatment techniques (IMSRT) and proton radiotherapy (IMPT).

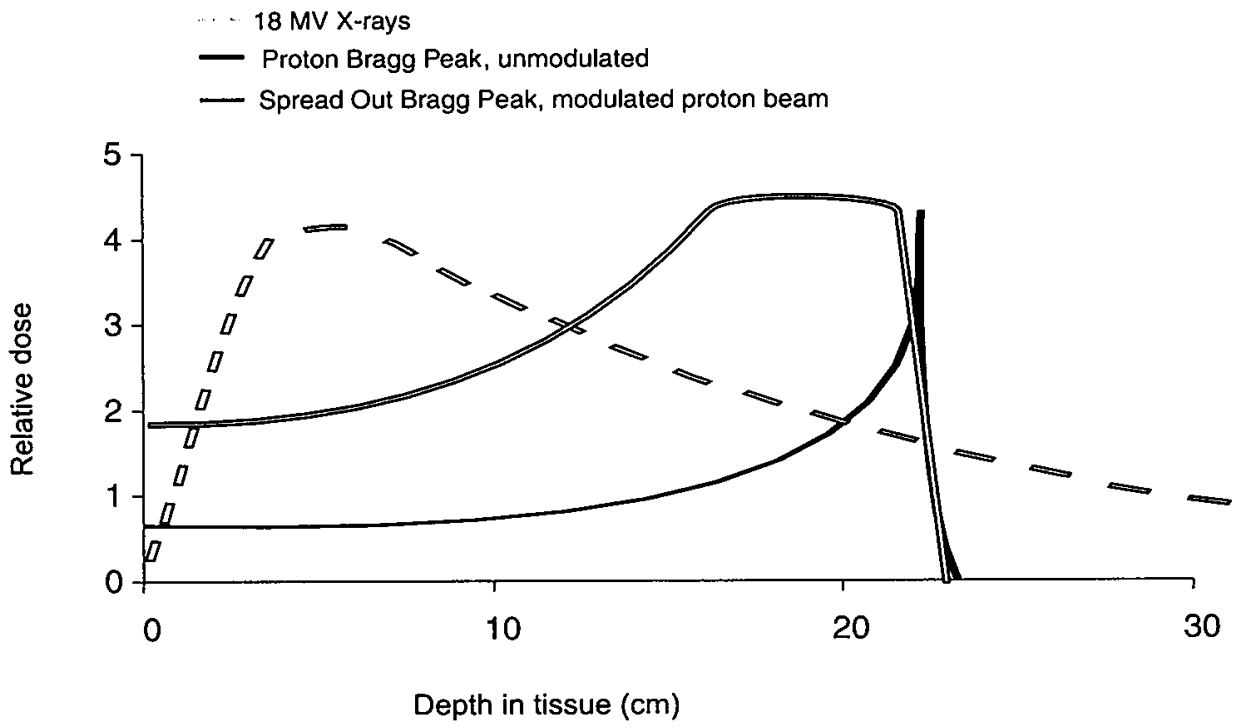

Figure 5. Comparison of depth dose distributions for different photon beams with that for a proton beam. Proton beams have no exit dose, whereas the dose deposited by a photon beam decreases exponentially with the depth in tissue. Curves are not drawn to scale. 
Classical clinical indications for proton therapy are tumours in close proximity to critical structures, especially those partially enclosed by the tumour itself, and tumours needing a high dose of irradiation for local control, for example lesions in the base of skull. With intensity modulation now clinically available, the same indications apply for radiotherapy with photons ${ }^{56}$. IMRT has been shown to have the ability to conform the dose to concavities and to avoid critical organs to an extent, which is not achievable with conventional means ${ }^{2,73}$.

\section{SPARING OF NORMAL TISSUE}

Normal tissues are tissues not suspected of tumour involvement. It is a fact that a reduction of dose to a defined volume of a critical organ or normal tissue will result in a lower risk of toxicity and morbidity. Three-dimensional CRT aims to reduce the dose delivered to organs-at-risk (OAR) to a minimum possible. The relationship between tolerance and volume of normal tissue irradiated is tissue and dose dependent: Cranial nerves are best spared by fractionation. This is best known for optic nerves and the chiasm. If the daily fraction size is kept to $\leq 1.9$ Gy the risk of optic nerve injury is low ( $<2 \%$ at 10 years), whereas the risk of damage is significantly increased with fraction sizes of $\geq 2 \mathrm{~Gy}^{22,55}$. Threshold values for late toxicity expressed as endocrinopathy were recently published for the pituitary gland and the hypothalamus. A higher dose than 50 Gy to the pituitary gland and the hypothalamus has been described to increase the risk of the incidence of an endocrinopathy $(75 \%$ at 5 years for patients receiving $\geq 50$ Gy compared to $33 \%$ receiving $<50 \mathrm{~Gy})^{54}$.

Cancer induced by radiation is assumed to be a function of dose. Zuccarello et al. have reported on low doses inducing meningioma after irradiation of the tinea capitus ${ }^{82}$. A significantly higher number of secondary malignancies in volumes receiving $<6 \mathrm{~Gy}$ has been reported by Doerr et al. ${ }^{13}$. A 23 -fold increase in risk of radiation-induced brain tumours in children with leukaemia treated with cranial radiotherapy has been reported ${ }^{52}$. Induced cancers may arise not only in low dose regions, but also in high dose volumes. Several cases of carcinogenesis following radiosurgery have been reported $^{45}$. Hence another aim of CRT is the sparing of normal tissue by decreasing the integral dose.

\section{CLINICAL OUTCOME}

The question of clinical impact of higher conformality needs to be addressed in clinical studies. The main indications for using highly conformal treatments are the 
reduction of dose to normal tissue and organs-at-risk and the possibility of a dose escalation. A reduction of dose to normal tissue implies a reduction of early and late toxicity on the one hand, but on the other hand, a reduction of safety margins (and thus the irradiated volume) may carry the risk of a geographical miss and reduced local control. The main curative indications for SCRT are benign and paediatric brain tumours both carrying an excellent long-term prognosis. A dose escalation may have its place for indications where local tumour control is not satisfactory or poor, as for malignant glioma. Radiosurgery has its classical place in the treatment of brain metastases.

Dose escalation in the treatment of malignant glioma

High-grade gliomas are the most common of all CNS tumours. Primary treatment options include surgical resection and radiotherapy. The effectiveness of radiotherapy in terms of survival benefit has been demonstrated in prospective randomised studies, $56-78$. There is evidence of a dose response relationship for doses below 60 $\mathrm{Gy}^{5}$. Local control of high-grade glioma may be achieved by increasing the dose to the tumour without increasing the dose to normal brain tissue as tumours almost always recur locally. Consequently, 3D-CRT in the form of radiosurgery ${ }^{66}$ or SCR $T^{64}$, protons ${ }^{17}$ or an integrated intensity modulated boost ${ }^{69}$ were proposed for dose escalation to doses $>70 \mathrm{~Gy}$. Many of these studies showed a promising median survival of $20-27$ months, but with an eventual increased risk of necrosis ${ }^{17,63}$. Feasibility studies have shown this risk to be lower with fractionated SCRT in contrast to radiosurgery ${ }^{42}$.

\section{Optic nerve sheath meningioma}

Optic nerve sheath meningiomas (ONSM) represent $1-2 \%$ of all meningiomas and $35 \%$ of all optic nerve tumours. They can originate in the orbit (primary OSNM) or grow into the orbit from an intra-cranial lesion (secondary ONSM). Most cases are unilateral, $5 \%$ are bilateral. Eighty per cent of them occur in females. Characteristically, their natural history is that of a slow indolent pattern of growth resulting in gradual progressive and unremitting loss of vision. Proptosis, disturbance of eye motility, progressive loss of vision and lid oedema are the leading symptoms ${ }^{8,14}$. ONSM are not associated with mortality or primary neurological morbidity other than visual loss. Treatment decisions should therefore take these issues into account in choosing the best treatment option of observation to surgery or radiotherapy. 


\section{Low-grade glioma in children}

Primary central nervous system (CNS) tumours are the most common solid tumours of childhood. Most are of glial origin with approximately $80 \%$ of low grade (WHO classification grade I and II). Pilocytic astrocytomas (WHO grade I) in children are rare and predominantly located in the cerebellum, optic pathways, hypothalamic area or in the third ventricular region ${ }^{20}$. They carry an excellent long-term prognosis in paediatric patients ${ }^{19,58}$. Currently, complete surgical excision is the treatment of choice. However, some low-grade glioma (LGG) are not amenable to a complete surgical resection at presentation without unacceptable morbidity. In these cases, a surveillance policy is usually recommended and treatment initiated at the time of progression. For inoperable and progressive paediatric LGG localised radiotherapy is at present perceived as the gold standard in the management of these patients. Radiotherapy was shown to be an effective treatment modality with long-term tumour control rates of $80-90 \%{ }^{31}$. Late sequelae of radiotherapy as for example neurocognitive deficits and the development of second malignant neoplasms are of concern in treating children with a long life expectancy. Apart from its technical advantages, SCRT also has the potential to reduce late sequelae. A reduction in the volume of normal brain tissue irradiated to high doses has been shown to reduce long-term neurocognitive sequelae ${ }^{25}$.

\section{OBJECTIVES OF THE THESIS}

This thesis deals with the technical, physical and clinical aspects of full three-dimensional cranial stereotactic radiotherapy and its implementation in the clinic. It considers the different treatment techniques and investigates the application of SCRT in the treatment of specific tumours and its clinical outcome.

\section{Specific aims}

The goal of any highly conformal irradiation technique such as SCRT is a high degree of dose conformation to the target volume. The higher the degree of conformation, the greater the set-up accuracy required to reduce the amount of normal tissue irradiated. Part I of this thesis deals with the quantification of the accuracy and reproducibility of patient's repositioning for SCRT (Chapter 2).

Despite the existence and use of different conformal 3D treatment techniques and complex approaches to radiotherapy treatment planning, it is unclear if one approach is superior to another, or if certain circumstances would favour one approach rather than another. A $3 \mathrm{D}$ conformal treatment technique may be superior to conventional 
radiation therapy in the irradiation of brain tumours, because many lesions are irregularly shaped and situated closely to critical structures. Since IMRT has become clinically available it seems logical to use it in the treatment of brain tumours or to combine it with stereotactic conformal radiotherapy. Thus, Part II of this thesis deals with the question of optimisation of treatment plans using comparative studies looking into dose distributions of different treatment and planning techniques of SCRT, IMSRT, proton beams and IMPT. This was done in order to investigate whether or not there is a benefit in using proton beams and intensity modulation for tumours with an irregular shape and concavities, which often harbour OAR such as the brain stem or the pituitary (Chapters $3-5$ ).

Part III of this thesis deals with the implementation of stereotactic radiotherapy in the clinic looking at its clinical outcome for different tumour entities. Questions investigated here are whether an escalation of dose by means of a fractionated stereotactic boost after conventional radiotherapy is safe and can improve local control. This is assessed with respect to survival, local control and toxicity in the initial management of a selected patient group with malignant glioma (Chapter 6).

In a further investigation it was assessed whether stereotactic radiotherapy can improve or maintain vision in a defined group of patients harbouring primary optic nerve sheath meningioma. The endpoints were visual function and symptom and tumour control (Chapter 7).

The questions were investigated whether it is safe to reduce the safety margin added for treatment with respect to local tumour control and whether the reduction of irradiated normal brain tissue to a higher dose can reduce late side effects in children harbouring inoperable or recurrent low grade glioma (Chapter 8).

\section{REFERENCES}

1. Alheit $\mathrm{H}$, Dornfeld $\mathrm{S}$, Dawel $\mathrm{M}$, $c$ t al. Patient position reproducibility in fractionated stereotactically guided conformal radiotherapy using the BrainLab mask system. Strahlenther Onkol 2001;177:264-268.

2. Benedict $\mathrm{SH}$, Cardinale $\mathrm{RM}, \mathrm{Wu} \mathrm{Q}$, et al. Intensity-modulated stereotactic radiosurgery using dynamic micro-multileaf collimation. Int R Radiat Oncol Biol Phys 2001;50(1):751-758.

3. Bentzen SM. High-tech in radiation oncology: should there be a ceiling? Int J Radiat Oncol Biol Phys 2004;58(2):320-330.

4. Berrino F, Capocaccia R, Estève J, ct al. Survival of Cancer Patients in Europe: The EUROCARE-2 Study. LARC Scientific Publications No. 151. Lyon, International Agency for Research on Cancer, 1999. 
5. Bleehen NM and Stenning SP. A Medical Cancer Research Council trial of two radiotherapy doses in the treatment of grades 3 and 4 astrocytoma. The Medical Research Council Brain Tumour Working Party. Br J Cancer 1991;64:769-774.

6. Bourland JD and McCollough KP. Static field conformal stereotactic radiosurgery: Physical techniques. Int J Radiat Oncol Biol Phys 1993;28:471-479.

7. Brahme A. Individualizing cancer treatment: biological optimization models in treatment planning and delivery. Int J Radiat Oncol Biol Phys 2001;49(2):327-337.

8. Cantore WA. Neural orbital tumors. Cur Opin Ophthalmol. 2000 Oct;11(5):367-571.

9. Cardinale RM and Karvanagh, BD. Conformal radiotherapy: what is it and why does it matter? Surgical Oncology Clinics of North America 2000;9(3):415-434.

10. Cardinale $\mathrm{RM}$, Benedict $\mathrm{SH}, \mathrm{Wu} \mathrm{Q}$, et al. A comparison of three stereotactic radiotherapy techniques; arcs vs. non-coplanar fixed fields vs. intensity modulation. Int J Radiat Oncol Biol Pliys 1998;42(2),431-436.

11. Dahl O, Kardamakis D, Lind B and Rosenwald JC. Current status of conformal radiotherapy. Acta Oncologica 1996;35(Suppl 8):41-57.

12. Daisne JF, Dupres TH, Weynand B, ef al. Tumor Volume in Pharyngolaryngeal Squamous Cell Carcinoma: Comparison at CT, MR Imaging, and FDG PET and Validation with Surgical Specimen. Radiology. 2004 Aug 18 (Epub ahead of print)

13. Doerr $W$ and Hermann $T h$. Second primary tumors after radiotherapy for malignancies. Strallenther Onkol 2001;178:357-62.

14. Dutton JJ. Optic nerve sheath meningiomas. Sur, Ophthalmol 1992;37:167-183.

15. Egger E, Schalenbourg A, Zografos L, ct al. Maximizing local tumor control and survival after proton beam radiotherapy of uveal melanoma. Int $J$ Radiat Oncol Biol Phys 2001;51:138-147.

16. European Network of Cancer Registries. EUROCIM version 5.0, Lyon 2002

17. Fitzek MM, Thornton AF, Rabinov JD, et al. Accelerated fractionated proton/photon irradiation to 90 cobalt gray equivalent for glioblastoma multiforme: results of a phase II prospective trial. J Ncurosurg 1999;91:251-260.

18. Fuks $\mathrm{Z}$ and Horwich A. Clinical and technical aspect of conformal therapy. Radiother Oncol 1993;29:219.

19. Gajjar A, Sanford RA, Heideman R, ct al: Low-grade astrocytoma: A decade of experience at St. Jude Children's research hospital. J Clin Oncol 1997;15(8):2792-2799.

20. Giannini $C$ and Scheithauer BW. Classification and grading of low-grade astrocytic tumors in children. Brain Pathology 1997;7:785-798.

21. Gill SS, Thomas GT, Warrington AP and Brada M. Relocatable frame for stereotactic external beam radiotherapy. Int J Radiat Oncol Biol Phys 1991;20:599-603.

22. Goldsmith BJ, Rosenthal SA, Wara WM, et al. Optic neuropathy after irradiation of meningioma. Radiology 1992;185:71-76.

23. Graham JD, Nahum AE and Brada M. A comparison of techniques for stereotactic radiotherapy by linear accelerator based on 3-dimensional dose distributions. Radiother Oncol $1991 ; 22(1): 29-35$. 
24. Graham JD, Warrington AP, Gill SS and Brada M. A non-invasive, relocatable stereotactic frame for fractionated radiotherapy and multiple imaging. Radiother Oncol 1991;21:60-62.

25. Gregor A, Cull A, Traynor E, et al. Neuropsychometric evaluation of long-term survivors of adult brain tumours: Relationship with tumour and treatment parameters. Radiother Oncol 1996;41:55-59

26. Gross MW, Weber WA, Feldmann HJ, Bartenstein P, Schwaiger M and Molls $M$. The value of F-18-fluorodeoxyglucose PET for the 3-D radiation treatment planning of malignant gliomas. Int J Radiat Oncol Biol Phys 1998;41:989-995.

27. Hamilton RJ, Kuchnir FT, Pelizzari CA, Sweeney PJ and Rubin SJ. Repositioning accuracy of a non-invasive head fixation system for stereotactic radiotherapy. Med Phys 1996; 23:1909-1917.

28. Hamilton RJ, Kuchnir FT, Sweeney P, et al. Comparison of static conformal field with multiple non-coplanar arc techniques for stereotactic radiosurgery or stereotactic radiotherapy. Int J Radiat Oncol Biol Phys 1995;33(5):1221-1228.

29. Herholz K, Holzer T, Bauer B, et al. 11C-methionine PET for differential diagnosis of low-grade gliomas. Neurology 1998;50(5):1316-1322.

30. Horsley $\mathrm{V}$ and Clarke $\mathrm{RM}$. The structure and function of the cerebellum examined by a new method. Brain 1908;31:45-124.

31. Horwich A and Bloom HJ. Optic gliomas: radiation therapy and prognosis. Int J Radiat Oncol Biol Phys 1985;11:1067-1079.

32. Horwitz EM and Hanks GE. Three-dimensional conformal radiation therapy: what are the costs and benefits? Surgical Oncology Clinics of North America 2000;9(3):455-467.

33. Kalapurakal JA, Ilahi Z, Kepka A, ct al. Repositioning accuracy with the Laitinen frame for fractionated stereotactic radiation therapy in adult and pediatric brain tumours: preliminary report. Radiology 2001;218:157-161.

34. Karger CP, Jäkel O, Debus J, Kuhn S and Hartmann GH. Three-dimensional accuracy and interfractional reproducibility of patient fixation and positioning using a stereotactic head mask system. Int J Radiat Oncol Biol Phys 2001;49:1493-1504.

35. Khoo VS, Adam EJ, Saran F, Bedford JL, Perks JR, Warrington AP and Brada M. A comparison of clinical target volumes determined by $\mathrm{CT}$ and $\mathrm{MRI}$ for the radiotherapy planning of base skull meningiomas. Int J Radiat Oncol Biol Phys 2000;46(5):1309-1317.

36. Kleihues P, Cavenee WK (eds). Tumours of the nervous system. Pathology \& Genetics. In: World Health Organization Classification of Tumours. IARC Press, Lyon, 2000.

37. Koehler $\mathrm{AM}$, Preston $\mathrm{AB}$ and Preston WM. Protons in radiation therapy. Radiology 1972;104:191-195.

38. Kolitsi Z, Dahl O, Van Loon R, et al. Quality assurance in conformal radiotherapy: DYNARAD consensus report on practice guidelines. Radiother Oncol 1997;45(3):217-223.

39. Kooy HM, Dunbar SF, Tarbell NJ, et al. Adaptation and verification of the relocatable Gill-Thomas-Cosman frame in stereotactic radiotherapy. Int J Radiat Oncol Biol Phys 1994; 30:685-691.

40. Kubo HD, Pappas CT and Wilder RB. A comparison of arc-based and static mini-multileaf collimator-based radiosurgery treatment plans. Radiother Oncol 1997;45(1):89-93. 
41. Laing RW, Bentley R, Nahum AE, et al. Stereotactic radiotherapy of irregular targets: a comparison between static conformal beams and non-coplanar arcs. Radiother Oncol $1993 ; 28(3): 241-246$.

42. Laing RW, Warrington AP, Graham J, Britton J, Hines F and Brada M. Efficacy and toxicity of fractionated stereotactic radiotherapy in the treatment of recurrent gliomas (phase I/II study). Radiother Oncol 1993;27:22-29.

43. Leksell DG. Stereotactic radiosurgery. Present status and future trends. Neurol Res $1987 ;(2): 60-68$.

44. Leunens G, Menten J, Weltens C, Verstraete J and van der Schueren E. Quality assessment of medical decision making in radiation oncology: Variability in target volume delineation for brain tumours. Radiother Oncol 1993;29:169-175.

45. Loeffler JS, Niemierko A and Chapman PH. Second tumors after radiosurgery: Tip of an iceberg or a bump in the road? Ncurosurgery 2003;52(6):1436-1442.

46. Lomax AJ, Pedroni E, Schaffner B, et al. 3D treatment planning for conformal proton therapy by spot scanning. In: K Faulkner, B Carey, A Crellin, RM, eds. Quantitative Imaging in Oncology. Harrison. London: British Institute of Radiology;1996. pp 67-71.

47. Marks LB, Sherouse GW, Ewton JR, et al. Conformal radiation therapy with fixed shaped coplanar or non coplanar radiation beam bouquets: A possible alternative to radiosurgery. Int J Radiat Oncol Biol P/lys 1995;33:1209-1219.

48. Miralbell R, Lomax AJ, Borfeld T, Rousaud $M$ and Carrie M. Potential role of proton therapy in the treatment of pediatric medulloblastoma/primitive neuro-ectodermal tumors: spinal theca irradiation. Int J Radiat Oncol Biol Phys 1997;38:805-811.

49. Miralbell R, Lomax AJ, Borfeld T, Rousaud $M$ and Carrie M. Potential role of proton therapy in the treatment of pediatric medulloblastoma/primitive neuro-ectodermal tumors:reduction of the supratentorial target volume. Int J Radiat Oncol Biol Phys 1997;38:477-484.

50. Miranpuri AS, Tome WA, Paliwal BR, et al. Assessment of patient-independent intrinsic error for a non-invasive frame for fractionated stereotactic radiotherapy. Int $J$ Cancer 2001;20:320-325.

51. Murphy PS, Viviers L, Abson C, et al. Monitoring temozolomide treatment of low-grade glioma with proton magnetic resonance spectroscopy. British J Cancer 2004;90:781-786.

52. Neglia J, Meadows A, Robinson L, et al. Second malignant neoplasms after acute lymphoblastic leukemia in childhood. N Engl J Med 1991;325:1330-1336.

53. Paddick I. A simple scoring ratio to index the conformity of radiosurgical treatment plans. $J$ Neurosurg 2000; (Suppl 3) 93:219-222.

54. Pai HH, Thornton A, Katznelson L, ct al. Hypothalamic/pituitary function following high-dose conformal radiotherapy to the base of skull: demonstration of a dose-effect relationship using dose-volume histogram analysis. Int J Radiat Oncol Biol Phys 2001;49(4): 1079-1092.

55. Parsons JT, Bova FJ, Mendenhall WM, ct al. Response of the normal eye to high dose radiotherapy. Oncolog $\gamma 1996 ; 10: 837-848$. 
56. Pirzkall A, Debus J, Haering $P$, ct al. Intensity modulated radiotherapy (IMRT) for recurrent, residual, or untreated skull-base meningiomas: preliminary clinical experience. Int J Radiat Oncol Biol Phys 2003;55(2):362-372.

57. Pirzkall A, Nelson SJ, McKnight TR, et al. Metabolic imaging of low-grade gliomas with three-dimensional magnetic resonance spectroscopy. Inter J Radiat Oncol Biol Phys 2002;53(5):1254-1264.

58. Pollack IF, Claassen D, Al-Shboul Q, et al. Low-grade gliomas of the cerebral hemispheres in children: an analysis of 71 cases. J Neurosurg 1995;82:536-547.

59. Purdy JA. Three-dimensional physics and treatment planning, in Principles and Practice of Radiation Oncology, C. A. Perez \& L. W. Brady (eds), Lippincott-Raven Publishers, Philadelphia, 1997. pp. 343-369.

60. Ries LAG, Eisner MP, Kosary CL, Hankey BF, Miller BA, Clegg L, Mariotto A, Feuer EJ, Edwards BK (eds). SEER Cancer Statistics Revieu, 1975-2001, National Cancer Institute. Bethesda, MD, http://seer.cancer.gov/csr/1975_2001/, 2004.

61. Rosenthal ST, Gall KP, Jackson M and Thornton AF. A precision cranial immobilization system for conformal stereotactic fractionated radiation therapy. Int J Radiat Oncol Biol Phys 1995;33:1239-1245.

62. Salter BJ, Fuss M, Vollmer DG, et al. The TALON removable head frame system for stereotactic radiosurgery/radiotherapy: measurement of the repositioning accuracy. Int $J$ Radiat Oncol Biol Phys 2001;51: 555-562.

63. Shenouda G, Souhami L, Podgosark EB, et al. Radiosurgery and accelerated radiotherapy for patients with glioblastoma. Can J Neurol Sci 1997;24:110-115.

64. Shepherd SF, Laing RW, Cosgrove VP, et al. Hypofractionated stereotactic radiotherapy in the management of recurrent glioma. Int J Radiat Oncol Biol Phys 1997;37:393-398.

65. Shiu AS, Kooy HM, Ewton JR, et al. Comparison of miniature multileaf collimation (MMLC) with circular collimation for stereotactic treatment. Int J Radiat Oncol Biol Phys 1997;37(3):679-688.

66. Shrieve DC, Alexander 3rd E, Black PM, et al. Treatment of patients with primary glioblastoma multiforme with standard postoperative radiotherapy and radiosurgical boost: prognostic factors and long-term outcome. J Ncurosurg 1999;90:72-77.

67. Suit HD and Goitein M. Rationale for use of charged particle and/or fast neutron beams in radiation therapy. I $n$ Radiation Biology in Cancer Research, Meyn RE, Withers HR (eds). New York: Raven Press, 1980, pp 547-565.

68. Suit $\mathrm{HD}$ and Urie M. Proton Beams in Radiation Therapy. Journal Natl Cancer Inst 1992;84:155-164.

69. Thilmann C, Zabel A, Grosser KH, Hoess A, Wannenmacher M and Debus J. Intensity-modulated radiotherapy with an integrated boost to the macroscopic tumour volume in the treatment of high-grade gliomas. Int J Cancer 2002;96:341-349.

70. Thornton Jr. AF, Hegarty TJ, Ten Haken Rk, ct al. Three-dimensional treatment planning of astrocytomas: A dosimetric study of cerebral irradiation. Int J Radiat Oncol Biol Phys 1991;20:1309-1315. 
71. Tralins KS, Douglas JG, Stelzer KJ, et al. Volumetric analysis of 18F-FDG PET in glioblastoma multiforme: prognostic information and possible role in the definition of target volumes in radiation dose escalation. J Nud Med 2002;43(12):1667-1673.

72. Tsuyuguchi N, Sunada I, Iwai Y, et al. Methionine positron emission tomography of recurrent metastatic brain tumor and radiation necrosis after stereotactic radiosurgery: is a differential diagnosis possible? J Neurosurg 2003;98(5):1056-1064.

73. Verhey LJ. Comparison of Three-Dimensional Conformal radiation therapy and intensity-modulated radiation therapy systems. Seminars Radiat Oncol 1999, 9:78-98.

74. Vernimmen FJ, Harris JK, Wilson J, et al. Stereotactic proton beam therapy of skull base meningiomas. Int J Radiat Oncol Biol Phys 2001;49(1):99-105.

75. Visser O, Coebergh JWW, Schouten LJ, Dijck JAAM van (eds). Incidence of Cancer in the Netherlands 1997. Utrecht: Vereniging van Integrale Kankercentra, 2001.

76. Walker MD, Green SB, Byar DP, et al. Randomized comparisons of radiotherapy and nitrosoureas for the treatment of malignant glioma after surgery. N Eng J Med 1980;303: 1323-1329.

77. Walker MD, Strike TA and Sheline GE. An analysis of dose-effect relationship in the radiotherapy of malignant gliomas. Int J Radiat Oncol Biol Phys 1979;5:1725-1731.

78. Walker MD, Strike TA and Sheline GE. Evaluation of BCNU and/or radiotherapy in the treatment of anaplastic gliomas. A cooperative clinical trial.J Neurosurg 1978;49:333-343.

79. Webb St: Preface. In: Webb ST (eds). The Physics of Three-Dimensional Radiation Therapy. Conformal Radiotherapy, Radiosurgery and Treatment Planning. Bristol and Philadelphia: Institute of Physics Publishing; 1993 .pp. ix-xi.

80. Webb St: Chapter 4: The physics of proton radiotherapy. In: Webb St (eds). The physics of three-dimensional radiation therapy. Bristol and Philadelphia: Institute of Physics Publishing; 1993 .pp.172-212.

81. Wong VY, Tung Sy, Leung TW and Ho KH. CT verification of isocentre relocatability using stereotactic mask fixation system. Clin Oncol ( $R$ Coll Radiol) 2003;15:280-287.

82. Zuccarello $M$, Sawaya $R$ and deCourten-Meyers $G$. Glioblastoma occurring after radiation therapy for meningiomas: Case report and review of literature. Neurosurgery 1986;19: 114-119. 


\section{PART I}

\section{Assessment of accuracy}


CHAPTER 2

Repositioning accuracy of fractionated stereotactic irradiation: Assessment of isocentre alignment for different dental fixations by using sequential CT scanning

Brigitta G. Baumert, Peter Egli, Stephan Studer, Cary Dehing and J. Bernard Davis

Radiotherapy and Oncology 2004; in press. 


\section{Abstract}

Background and purpose: To quantify the accuracy and reproducibility of patient repositioning in fractionated stereotactic conformal radiotherapy (SCRT) using dental fixations in conjunction with a stereotactic head mask.

Patients and Methods: One hundred and fourteen verification CT scans were performed on 57 patients in order to check set-up alignment. The first scan was done immediately after the first treatment. Twelve patients were checked for alignment accuracy with weekly CT scans over a period of $3-6$ weeks, all others had $1-2$ scans. Two different dental fixations were used in combination with a non-invasive mask system: an upper jaw support (35 patients) and a customised bite-block (17 patients). Five patients were treated with no additional fixation. Co-registration to the planning CT was used to assess alignment of the isocentre to the reference markers. Additionally, the intra-operator variability of image co-registration was assessed.

Results: There was a significant improvement of the overall alignment in using the bite block instead of the upper jaw support $(p<0.001)$. The mean deviation was for the bite block $2.2 \pm 1.1 \mathrm{~mm}$ ( $1 \mathrm{SD}$ ), for the upper jaw support $3.3 \pm 1.8 \mathrm{~mm}$ and $3.7 \pm$ $2.8 \mathrm{~mm}$ for the mask alone. Overall isocentre deviations independent of the method of fixation were $2.8 \mathrm{~mm}$ (1.7 mm, 1SD). Displacements in CC direction were significantly less for the bite-block compared to the upper jaw support $(p=0.03)$. The addition of an upper jaw support significantly reduced lateral rotations compared to the mask system alone $(p=0.03)$. The intra-operator variability of image co-registration was $1.59 \pm 0.49 \mathrm{~mm}(1 \mathrm{SD})$.

Conclusion: The reproducibility of patient positioning using a relocatable head mask system combined with a bite block is within the reported range for similar devices and is preferable to a simple upper jaw support. In order to further reduce the margin for the planning target volume an intra-oral dental fixation is recommended.

Key words: sequential CT scans, stereotactic radiotherapy, immobilization, patient repositioning, dental tray, bite-block, mouth plate, relocatable frame 


\section{INTRODUCTION}

In the use of stereotactic conformal radiotherapy (SCRT) accuracy is necessary in order to reduce safety margins around the tumour. This in turn reduces the volume of normal tissue receiving a high dose of radiation. SCRT combines the precision of the stereotactically guided tumour localisation and the radiobiological advantages of fractionation. This implies the necessity for a highly accurate repeated alignment of the planned isocentre with the isocentre of the linear accelerator.

A simulator can be used to simulate each field or for checking the position of the isocentre $1,12,15$. However, small inaccuracies in the alignment of the crosshairs and beam defining wires in the simulator head will be magnified on the simulator image. Thus, even if the simulator isocentre accuracy is very good $(<2 \mathrm{~mm}$ diameter) small inaccuracies in the beam-defining wires may make it difficult to assess the correct position of the field with respect to the anatomy. In contrast, the tertiary beam collimation (e.g. micro-multileaf collimators) used in SCRT is much closer to the patient. This can be at approximately $30 \mathrm{~cm}$ from the isocentre and is thus less sensitive to small inaccuracies in alignment.

One way of checking the correct position of the isocentre in the patient is to repeat the planning computer tomography (CT) scan at regular intervals after the first set-up and assess the alignment of the isocentre marks on the mask with the planned isocentre. This technique has been reported on by several groups ${ }^{10,14,18,22,23}$. The observed mean set-up accuracy varied between $0.3-2.3 \mathrm{~mm}$ depending on the type of fixation used.

For this study a commercially available mask was used with the addition of two fixations. A simple upper jaw support and a customised bite-block, were assessed after antero-posterior and rotational head movements were observed when using the standard mask. The present work reports on the use of check CT scans to assess accuracy of alignment of such a mask in conjunction with each of the two additional fixations.

\section{MATERIAL AND METHODS}

\section{Overview}

Fractionated SCR T has been used for the treatment of malignant and non-malignant lesions of the brain at the University Hospital Zurich since 1994. With the acquisition of a dedicated CT scanner for Radiation Oncology in April 2000, it was possible to check repositioning accuracy by serial CT scanning. A simulation is not included 
into the stereotactic treatment protocol as the precision of stereotaxy is considered to exceed the positional accuracy obtained by conventional simulation. Instead, serial CT scanning is used to verify the accuracy of treatment set-up. In total 57 patients were assessed this way. The number of CT scans for each patient differed according to the fractionation schedule. The first patients were checked by weekly scans in order to assess the accuracy of the system as a whole. For a hypofractionation schedule with 4-6 fractions, one check CT scan was done after the first fraction only. For a conventional fractionation schedule, e.g. 30 fractions, CT scans were done three times during treatment, immediately after the first fraction, after one-third and after two-thirds of the prescribed dose. The correction protocol was as follows: if the alignment accuracy was within $2 \mathrm{~mm}$, no adjustment was made. If a misalignment of 2-4 $\mathrm{mm}$ was detected, the co-ordinates of the planned isocentre were adjusted by half the measured values. If the misalignment was $>4 \mathrm{~mm}$, the planning was repeated with a recalculation of isodoses and check $C T$ scans were done at shorter intervals.

\section{Head fixation}

Patients were treated in an open stereotactic ring in conjunction with a full immobilisation thermoplastic mask (BrainLAB ${ }^{\circledR}$, Heimstetten, Germany). Two different dental fixations were used in combination with the mask system: a commercially available upper jaw support from BrainLAB and a customised bite-block (Figure 1a). Five patients were treated with no additional fixation before the upper jaw support and the bite-block were available. For a treatment duration of several weeks a bite-block was added to the mask system, for a hypo-fractionated schedule an upper jaw support (Figure 1b) was chosen in view of the high costs and preparation time of the bite block. For edentulous patients the upper jaw support was used, as a customized bite-block is not suitable for this patient group. The custom made bite block is an intra-oral device prepared by a maxillofacial prosthodontist of the Cranio-Maxillofacial Surgery Clinic, University Hospital Zurich and is fabricated in polymethylmetacrylate resin (PMMA resin) (Figure 1c). In order to achieve an accurate fitting, a dental impression of the upper and lower arches together with a bite registration is mandatory during a dental visit. The upper jaw support consists of a rectangular piece of polyethelene-covered metal rod fixed to the stereotactic ring and supporting the middle part of the upper dentition (Figure 1b).

\section{Planning and verification $C T$ scanning}

A CT scan of a slice thickness of $3 \mathrm{~mm}$ was taken in the treatment position using the stereotactic localizer. For very small tumours $2 \mathrm{~mm}$ slices were used. The data were transferred to the dedicated stereotactic treatment planning system (TPS) for treatment planning. The calculated position of the isocentre is transferred from the TPS to 

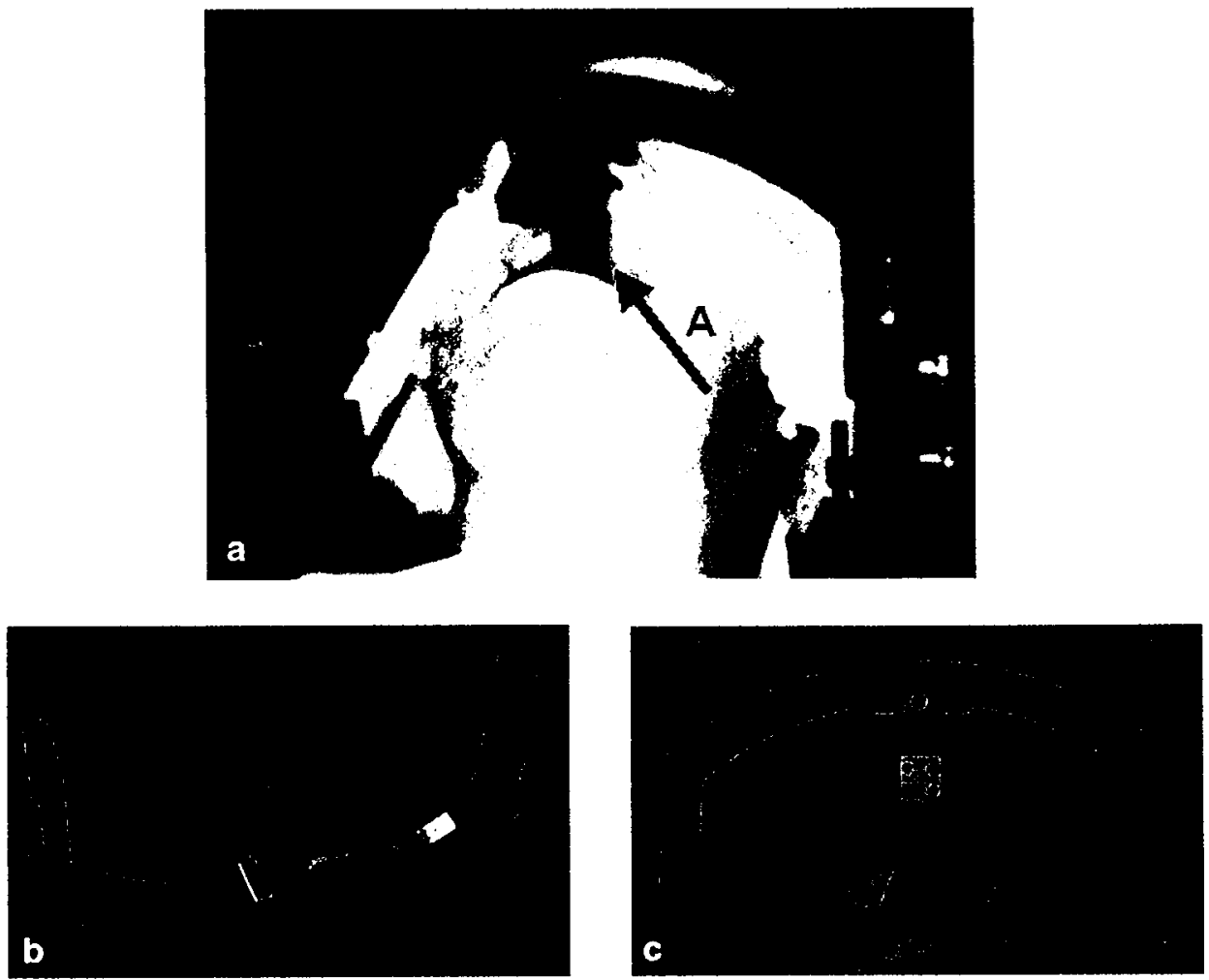

Figure 1. Head fixation system with a thermoplastic mask secured laterally to a frame, which is open anteriorly (1a). The upper jaw support or bite-block (A) is fixed to a detachable part of the frame (B). This part is removed for patient access. Figure $1 \mathrm{~b}$ ) shows the detached part of the frame with an upper jaw support, Figure 1c) the bite-block attached to the same frame.

the target localizer box, which is used to define the isocentre for each treatment session. During the first treatment, the position of the lateral and ceiling lasers defining the position of the isocentre are carefully marked on the immobilising mask with a fine pen. Immediately after the first fraction the patient is transferred to the scanner room and a first check CT scan is done in the treatment position to verify the position of the isocentre. The co-ordinates of the isocentre are obtained by using fine copper cross wires or later small metal beads ( $2 \mathrm{~mm}$ diameter) fixed on the mask at the exact position of the treatment room laser marks. The CT scan is repeated using exactly the same protocol as used for the planning CT scan. Data are transferred to the TPS for co-registration with the planning CT scan. 


\section{Intra-operator variability and image fusion}

The BrainLAB software (BrainSCAN V3.53 and V4.03) offers the possibility of semi-automatic image co-registration (fusion) of several data sets. As the version available at the beginning of the study was not very reliable, data sets from the first 32 patients were fused manually. Anatomical landmarks were used for reference. This operation is to some extent operator dependent. For this reason, the intra-operator variability was assessed by one operator fusing the same two data sets of the check CT and planning CT on six consecutive days without prior knowledge of the results of the previous fusion. CT data sets of the last 25 patient were fused using the BrainSCAN automatic fusion software.

\section{Measurement of isocentre position and definition of displacements}

Assessment of the isocentre alignment was done by superimposing the check CT scans on the planning CT scan. The centre of each cross-wire or bead was marked in a zoomed image and the co-ordinates of each point used to calculate the position of the point of congruence of the three markers, which corresponds to the position of the treatment isocentre. Displacements were assessed by calculating coordinates of the isocentre of the planning and check scans. Deviations were assessed in the $x, y$ and $z$ axes as medio-lateral (ML), antero-posterior (AP) and cranio-caudal (CC) deviations, respectively for translational displacements. Correspondingly, rotational displacements are defined as lateral, antero-posterior and cranio-caudal rotations of the $x, y$ and $z$ axes, respectively. The following formulae based on simple geometrical transitions were used:

where the vector $r 4=\left(r_{4}, r 4_{y}, r_{z}\right)$ corresponds to the position of the check scan isocentre and where the vector $r 1, r 2, r 3$ correspond to the positions of the markers on the left, right and ventral (anterior) position on the mask.

$$
\begin{aligned}
& r 4_{i}=r 1_{i}+\frac{\left[\left(r 2_{x}-r 1_{x}\right) \cdot\left(r 3_{x}-r 1_{x}\right)+\left(r 2_{x}-r 1_{x}\right)\left(r 2_{x}-r 1_{x}\right) \cdot\left(r 2_{x}-r 1_{x}\right)\left(r 2_{x}-r 1_{x}\right)\left(r 2_{x}-r 1_{x}\right)\right]}{\left(r 2_{x}-r 1_{x}\right)^{2}+\left(r 3_{x}-r 1_{x}\right)^{2}+\left(r 3_{x}-r 1_{x}\right)^{2}} \\
& i=\{x, y, z\}
\end{aligned}
$$

The formula for calculating $\alpha$ the angle of rotation (example for the cranio-caudal rotation) is:

$$
\alpha=\arctan \left[\frac{r_{1_{x}} r_{2_{x}}}{\left|r_{y}\right|+\left|r 2_{y}\right|}\right] \cdot \frac{360^{\circ}}{2 \cdot \pi}
$$

The overall translational displacement between the planned isocentre and the position of the treatment isocentre was calculated with a three-dimensional vector (3D 
vector) using the formula $\sqrt{x^{2}+y^{2}+z^{2}}$ where $x, y$ and $z$ represent the deviation in each direction.

Data were analysed directional dependent, i.e. taking into account negativity and positivity of each displacement. Positive $x$ values indicated a lateral movement or a rotation to the right, positive $y$ values represented an anterior movement or an anterior rotation, and positive $z$ values represented a cranial movement or a rotation.

\section{Statistics}

Data were analysed with StatView for Windows from Abacus Concepts, Inc., Version 4.57. Differences between groups were tested using the Kruskal-Wallis-Test and the Mann Whitney $U$ Test depending on the number of variables tested. A $\mathrm{p}$-value of $<0.05$ is considered significant at the $<95 \%$ confidence interval. The analysis was based on the hypothesis that all fixation combinations were equally good. In order to define significant differences between the mode of fusion (manual versus automatic image fusion) and method of fixation (e.g. upper jaw support versus bite-block) simultaneously the ANOVA test was used.

\section{RESULTS}

\section{General}

One hundred and fourteen scans of 57 patients were assessed. Of these, 35 patients (57 scans) were treated with the mask and an upper jaw support and 17 patients (50 scans) with the mask and a bite-block. In 5 patients ( 7 scans) no additional fixation was used. Twenty-six patients had 1 check scan, 19, 6, 2 and 4 patients had 2, 3, 4 and 6 scans, respectively.

\section{Isocentre alignment}

The total deviation independent of the method of fixation expressed by the3D vector was $2.8 \pm 1.7 \mathrm{~mm}(1 \mathrm{SD})$. The SD are largest for the cranio-caudal displacement and lateral rotation $\left(2.3 \mathrm{~mm}\right.$ and $\left.1.3^{\circ}\right)$ including the largest range of displacements $\left(-7.1-+6.9 \mathrm{~mm}\right.$ and $\left.-2.7-+4.6^{\circ}\right)$. The SD was $<1 \mathrm{~mm}$ or $<0.3$ degree for translational and rotational displacements, respectively. 
Table 1. Assessment of alignment of the 3 fixation systems in $x, y$ and $z$ directions. $P$-values correspond to comparisons between the pairs of fixations in the first column (Bite-block versus upper jaw fixation; upper jaw fixation versus no additional fixation; no additional fixation versus bite-block).

\begin{tabular}{|c|c|c|c|c|c|c|c|}
\hline \multirow{2}{*}{$\begin{array}{l}\text { Fixation } \\
\text { system }\end{array}$} & \multirow[t]{2}{*}{ Error (mm) } & \multicolumn{3}{|c|}{ Translational displacements (mm) } & \multicolumn{3}{|c|}{ Rotational discrepancies $\left(^{\circ}\right)$} \\
\hline & & $\begin{array}{l}\text { Cranio- } \\
\text { caudal }\end{array}$ & $\begin{array}{l}\text { Medio- } \\
\text { lateral }\end{array}$ & $\begin{array}{l}\text { Antero- } \\
\text { posterior }\end{array}$ & $\begin{array}{l}\text { Cranio- } \\
\text { caudal }\end{array}$ & Lateral & $\begin{array}{l}\text { Antero- } \\
\text { posterior }\end{array}$ \\
\hline \multirow{3}{*}{$\begin{array}{l}\text { Bite- } \\
\text { block }\end{array}$} & Mean (1 SI) & $0.6(1.8)$ & $-0.04(1.4)$ & $-0.1(0.8)$ & $0.1(0.8)$ & 0.2 & $-0.02(0.8)$ \\
\hline & Range & -3.2 to +5.1 & $-3.610+2.4$ & -1.9 to +1.3 & -1.0 to +2.8 & -2.4 to +1.9 & -1.6 to +2.6 \\
\hline & $p$-value & 0.03 & ı.s. & n.s & n.s & n.s & n.s \\
\hline \multirow{3}{*}{$\begin{array}{l}\text { Upper } \\
\text { jaw } \\
\text { support }\end{array}$} & Mean (1 SI)) & $1.5(2.6)$ & $0.2(1.7)$ & $-0.6(1.4)$ & $0.2(0.9)$ & $-0.1(1.5)$ & $0.2(1.1)$ \\
\hline & Range & -7.1 to +6.9 & -3.2 to +3.8 & -3.9 to +3.3 & -1.5 to +2.8 & -2.7 to +4.6 & -2.1 to +3. \\
\hline & $p^{\text {-value }}$ & n.s. & n.s & n.s. & n.s & 0.03 & n.s \\
\hline \multirow[t]{3}{*}{ None } & Mcin (1 SI) & $0.07(3.1)$ & $-0.5(3.3)$ & $-0.7(1.4)$ & $0.2(1.5)$ & $1.6(1.7)$ & $-0.6(0.8)$ \\
\hline & Range & -5.2 to +3.2 & -6.8 to +4.2 & -2.4 to -0.9 & -1.5 to +2.1 & -0.9 to +3.1 & -1.7 to +0.4 \\
\hline & $p$-valuc & $n . s$ & n.s & $\mathrm{n} . \mathrm{s}$ & n.s & $(0.05)$ & n.s \\
\hline
\end{tabular}

Abbreviations: n.s, not significant.

Table 2. Comparison of overall displacement (3D vector) for dental fixation systems

\begin{tabular}{llll} 
Fixations compared & $P$ - value & Dental fixation & $\begin{array}{l}\text { Deviations (mm) } \\
\text { (mean, 1 SD, range) }\end{array}$ \\
Bite-block / Upper jaw support & $0.001(\mathrm{~s})$ & Bite-block & 2.2, SD 1.1, 0.4 - 5.5 \\
Upper jaw support / None & 0.97 & Upper jaw & 3.3, SD 1.8, 0.2-7.8 \\
None / Bite-Block & 0.2 & None & 3.7, SD 2.8,0.8-8.9 \\
\hline
\end{tabular}

Abbreviations: s: significant.

\section{Comparison of dental fixations}

Data of the verification CT scans were divided into three groups according the type of dental fixation used and compared with each other. In the CC direction, fixation with a bite-block results in significantly smaller displacement than with an upper jaw support $(\mathrm{p}=0.03)$ (Table 1). For rotational movement the lateral rotation is significantly reduced through the use of an upper jaw support compared to no additional dental fixation $(p=0.03)$. The mean value is largest in the ML direction ( $x$ axis) if no additional fixation device is used with $3.3 \mathrm{~mm}$ (Table 1). For the upper jaw support the range of deviations in the $z$ axis $(\mathrm{CC})$ is $>1 \mathrm{~cm}(-7.1-+6.9 \mathrm{~mm})$. The 3D vector shows that the use of a bite-block reduces significantly translational displacements compared to the upper jaw support $(p<0.001)$ (Table 2$)$.

\section{Intra- operator variability of image fusion}

The largest variations of the calculated displacement of the marker isocentre co-ordinates resulting from repeated manual image fusions by the same operator are seen for 
the $\mathrm{CC}$ direction (mean $1.55 \mathrm{~mm}$ ) and is indicative of the achievable accuracy in this direction by using manual image fusion. The overall variability of image fusion was $1.59 \pm 0.49 \mathrm{~mm}(1 \mathrm{SD})$. There was a significant difference for the 3D vector comparing the upper jaw support and the bite-block using the manual fusion or the automatic fusion $(p=0.0068$ and $p=0.0036)$. Therefore, both factors influenced the results.

\section{DISCUSSION}

The goal of a highly precise irradiation technique such as stereotactic conformal radiotherapy (SCRT) is a high degree of dose conformation to the target volume. The higher the degree of conformation, the greater the set-up accuracy required to reduce the volume of normal tissue irradiated. Many reports have been published indicating that the set-up accuracy of relocatable fixation systems is $\leq 2$ $\mathrm{mm}^{1,3,4,6-10,14-19,20,21,23}$. The reproducibility of patient repositioning using a non-invasive fixation system combined with a dental fixation is reported to be within the range of $0.4-2.3 \mathrm{~mm}^{3,4,6,9,15-17,20,21}$. The mean displacement observed in this study is within this range, $2.2 \pm 1.1 \mathrm{~mm}$ (1 SD). Results are difficult to compare due to the different calculation methods and definitions of accuracy used in the literature. For example, the overall magnitude of displacements is not always reported by a $3 \mathrm{D}$ vector or definitions of calculation methods are not fully described. However, detailed results are often reported and differences between fixation systems can be detected by comparing results separately for the $\mathrm{x}, \mathrm{y}$ and $\mathrm{z}$ axes. Two groups have reported on the same fixation system as in this study ${ }^{1,12}$. Alheit et al. reported a mean of $2.1 \mathrm{~mm}$ (3D vector) of 33 patients evaluated by simulator check films and portal imaging ${ }^{1}$. The measured maximum isocentre deviations were between -4.5 and +6 $\mathrm{mm}$, which is similar to this study for the mask alone. One of the reasons for the higher mean of $3.7 \mathrm{~mm}$ in our study is presumably due to the small group of patients evaluated, 5 versus 33 patients. On the other hand, the importance of the human factor may not be underestimated: a mask can be made differently by different technicians, but deviations can also be related to patient compliance (we observed the largest deviations with non-compliant patients). Lopatta et al. reported values for the medio-lateral, cranio-caudal and antero-posterior translations of $<1.5 \mathrm{~mm}$. These are in agreement with mean values of $<1.6 \mathrm{~mm}^{12}$.

In this same study, the hypothesis that an additional fixation of the upper jaw is needed in order to possibly reduce cranio-caudal movements, was first proven by a direct comparison of SCRT with a thermoplastic mask alone versus the mask combined with a custom-made mouthpiece for the upper dentition ${ }^{12}$. The authors reported a significant improvement of random set-up errors in all three spatial 
dimensions, but especially in the cranio-caudal direction ( $z$ axis). The same significant reduction in movements along the $z$ axis with the use of an additional dental fixation is demonstrated in this study, whereas the addition of an upper jaw support reduced the medio-lateral rotation significantly. The greatest displacements with up to $6.9 \mathrm{~mm}$ were seen in the cranio-caudal and medio-lateral direction with no additional fixation or with the upper jaw support, suggesting that a rotation in the sagittal plane around the simple upper jaw support was possible. Consequently, the lateral rotation was demonstrated to be significantly reduced with an individually fitted bite-block.

There are few publications using check CT scans for stereotactic set-up verification $10,14,18,21-23$. Of those, only two used repeated verification $\mathrm{CT}$ scans, one for patient-independent measurements with a head phantom ${ }^{14}$, and one for patients undergoing a 6 week treatment course ${ }^{18}$. Salter et al reported a slight deterioration of isocentre set-up accuracy over a 6 week course of treatment, where the accuracy during the last 3 weeks was worse than during the first 3 weeks of treatment ${ }^{18}$. This may have been due to patient compliance.

In our study, serial check CT scanning for verification necessitated the patient's relocation outside of the treatment room and therefore the use of a separate reference system. Checking displacements with repeated CT scans has the advantage of a high image resolution for anatomical details together with a high geometric accuracy. Set-up errors at the accelerator are not taken into account in our method as would be the case for portal imaging.

Other possible causes for misalignment could be due to the incorrect positioning of the jaw support or bite block, or the alignment of isocentre marker beads to the treatment room lasers or indeed differences between the treatment room and scanner room lasers. It is also advisable that the same person (or a defined group of persons) does both set-ups in order to further reduce set-up errors.

The localisation accuracy of stereotactic systems is not per se influenced by CT slice thickness as the position of the isocentre is numerically defined by the co-ordinate system. However, localisation of an exact point in the CT scan data (as needed for definition of the marker position) and the matching of the scans (interscan spacing) is dependent on slice thickness and is reported to be between 1.2 and $1.7 \mathrm{~mm}$ for slices of $1-1.5 \mathrm{~mm}^{2,5,11,13}$. Bucholz et al. ${ }^{2}$ measured a decrease of $23 \%$ in the mean error when the scan thickness was decreased from 5 to $1.5 \mathrm{~mm}$ and a $45 \%$ decrease when the interscan spacing was reduced from 3 to $0.5 \mathrm{~mm}$. Yeung et al. ${ }^{24}$ found a positional accuracy of CT localization of $0.9 \pm 0.3 \mathrm{~mm}$ for a slice thickness of $2 \mathrm{~mm}$. If the inaccuracy associated with treatment set-up is included, the mean cumulative error (posi- 
tional and set-up) and spatial error for a slice thickness of $2 \mathrm{~mm}$ is $1.2 \pm 0.5 \mathrm{~mm}$ and $1.8 \mathrm{~mm}$, respectively (compared to $2.0 \pm 0.6 \mathrm{~mm}$ and $2.9 \mathrm{~mm}$ for $4 \mathrm{~mm}$ slice thickness) ${ }^{24}$. Accordingly, this would translate into a cumulative and spatial error of 1.6 and $2.4 \mathrm{~mm}$ for the $3 \mathrm{~mm}$ slices used in this group of patients. Thus, the observed inaccuracy for measurements in the cranio-caudal direction ( $\mathrm{z}$ axis) seems to be at least partially dependent on the CT slice thickness used. In a search for possible reasons for the observed pronounced intra-operator variability in CC direction, the total group was divided into manual fusion and automatic fusion to perform a subgroup analysis comparing the bite-block and upper jaw support as well as the method of fusion. This revealed a significant difference for both modes of fusion and fixation for the 3D vector. Therefore, a possible influence of the image fusion itself on the significant result in CC direction cannot be excluded as both variables influence the outcome. However, we found very low mean values $(1 \mathrm{~mm})$ based on a direction dependent calculation in this study (i.e. considering negativity and positivity of measurements). This corresponds to a small systematic error of the fixation and imaging method used.

\section{CONCLUSIONS}

Serial CT can be used to assess the reproducibility of patient position in stereotactic radiotherapy. For the mask system and additional fixation used in this series of patients alignment is within the range of published data. The higher degree of accuracy achievable with a dental fixation is mandatory in order to reduce the margin for the PTV and hence the volume of normal brain tissue irradiated. Based on this analysis, a margin of $3 \mathrm{~mm}$ would seem to be sufficient for patients treated with a bite-block because it includes two SD of 2.2 which covers $>95 \%$ displacements. This value would also correct for possible errors due to CT slice thickness of $2-3$ $\mathrm{mm}$. A larger margin should be chosen if no intra-oral fixation is used.

Random set-up errors cannot be avoided, but can be kept to a minimum with a strict verification and correction protocol. Serial CT measurements for set-up verification have the advantage of a high in-plane resolution in combination with a geometrically accurate three-dimensional information. 


\section{REFERENCES}

1. Alheit $\mathrm{H}$, Domfeld S, Dawel $M$ ct al. Patient position reproducibility in fractionated stereotactically guided conformal radiotherapy using the BrainLab mask system. Strahlenther Onkol. 2001;177:264-268.

2. Bucholz RD, Ho HW and Rubin JP. Variables effecting the accuracy of stereotactic localization using computerized tomography. J Neurosurg. 1993;79:667-673.

3. Gill SS, Thomas GT, Warrington AP and Brada M. Relocatable frame for stereotactic external beam radiotherapy. Int J Radiat Oncol Biol Phys. 1991;20:599-603.

4. Graham JD, Warrington AP, Gill SS and Brada M. A non-invasive, relocatable stereotactic frame for fractionated radiotherapy and multiple imaging. Radiother Oncol. 1991;21:60-62.

5. Grunert P, Maurer J and Muller-Forell W. Accuracy of stereotactic coordinate transformation using a localisation frame and computed tomographic imaging. Part I. Influence of the mathematical and physical properties of the CT on the image of the rods of the localisation frame and the determination of their centres. Neurosurg Rev. 1999;22:173-87.

6. Hamilton RJ, Kuchnir FT, Pelizzari CA, Sweeney PJ and Rubin SJ. Repositioning accuracy of a noninvasive head fixation system for stereotactic radiotherapy. Med Phys. 1996; 23:1909-17.

7. Kalapurakal JA, Ilahi Z, Kepka A et al. Repositioning accuracy with the Laitinen frame for fractionated stereotactic radiation therapy in adult and pediatric brain tumours: preliminary report. Radiology. 2001;218:157-161.

8. Karger CP, Jäkel O, Debus J, Kuhn S and Hartmann GH. Three-dimensional accuracy and interfractional reproducibility of patient fixation and positioning using a stereotactic head mask system. Int J Radiat Oncol Biol Phys. 2001;49:1493-1504.

9. Kooy HM, Dunbar SF, Tarbell NJ et al. Adaptation and verification of the relocatable Gill-Thomas-Cosman frame in stereotactic radiotherapy. Int J Radiat Oncol Biol Phys. 1994; 30:685-691.

10. Kortmann RD, Becker G, Perelmouter J, Buchgeister $M$, Meisner Ch and Bamberg B. Geometric accuracy of field alignment in fractionated stereotactic conformal radiotherapy of brain tumors. Int J Radiat Oncol Biol Phys. 1999;43:921-926.

11. Kress J, Minohara S, Endo M, Debus J and Kanai T. Patient position verification using CT images. Med Phys. 1999;26:941-948.

12. Lopatta E, Liesenfeld SM, Bank P et al. Improved patient repositioning accuracy by integrating an additional jaw fixation into a high precision face mask system in stereotactic radiotherapy of the head. Strahlenther Onkol. 2003;179:571-575.

13. Maciunas RJ, Galloway RL and Latimer JW. The application accuracy of stereotactic frames. Neurosurgery. 1994;35:682-695.

14. Miranpuri AS, Tome WA, Paliwal BR $c t$ al. Assessment of patient-independent intrinsic error for a noninvasive frame for fractionated stereotactic radiotherapy. Int $\mathrm{J}$ Cancer. $2001 ; 20: 320-325$. 
15. Rosenberg I, Alheit $\mathrm{H}$, Beardmore $\mathrm{C}$, Lee KS, Warrington AP and Brada M. Patient position reproducibility in fractionated stereotactic radiotherapy: an update after changing dental impression material. Radiother Oncol. 1999;50(2):239-40.

16. Rosenthal ST, Gall KP, Jackson M and Thornton AF. A precision cranial immobilization system for conformal stereotactic fractionated radiation therapy. Int J Radiat Oncol Biol Phys. 1995;33:1239-1245.

17. Ryken TC, Meeks SL, Pennington EC et al. Initial clinical experience with frameless stereotactic radiosurgery: analysis of accuracy and feasibility. Int J Radiat Oncol Biol Phys. 2001;51:1152-1158.

18. Salter BJ, Fuss M, Vollmer DG et al. The TALON removable head frame system for stereotactic radiosurgery/radiotherapy: measurement of the repositioning accuracy. Int J Radiat Oncol Biol Phys. 2001;51: 555-562

19. Schlegel W, Pastyr O, Bortfeld Th, Gademann G, Menke $M$ and Maier-Borst W. Stereotactically guided fractionated radiotherapy: technical aspects. Radiother Oncol. 1993; 29:197-204.

20. Sweeney RA, Bale R, Auberger $T$ et al. A simple and non-invasive vacuum mouthpiece-based head fixation system for high precision radiotherapy. Strahlenther Onkol. 2002;177:43-47.

21. Warrington AP, Laing RW and Brada M. Quality assurance in fractionated stereotactic radiotherapy. Radiother Oncol. 1994;30:239-246.

22. Willner J, Flentje $M$ and Bratengeier K. CT simulation in stereotactic brain radiotherapy-analysis of isocenter reproducibility with mask fixation. Radiother Oncol. 1997;45:83-88

23. Wong VY, Tung Sy, Leung TW and Ho KH. CT verification of isocentre relocatability using stereotactic mask fixation system. Clin Oncol ( $R$ Coll Radiol). 2003;15:280-287.

24. Yeung D, Palta J, Fontanesi J and Kun L. Systematic analysis of errors in target loacalization and treatment delivery in stereotactic radiosurgery (SRS). Int J Radiat Oncol Biol Phys. 1993;28: 493-498. 


\section{PART II}

\section{Methodological investigations}




\section{CHAPTER 3}

Intensity modulated stereotactic radiotherapy

versus stereotactic conformal radiotherapy for the treatment of meningioma predominantly located in the skull base

Brigitta G. Baumert, Ian Norton and J. Bernard Davis

International Journal of Radiation Oncology, Biology, Physics 2003;57(2):580-92 


\section{Abstract}

Purpose: This study evaluates a possible advantage of intensity-modulated stereotactic radiotherapy (IMSRT) over stereotactic conformal radiotherapy (SCRT) in the treatment of lesions in the base of the skull.

Methods and Materials: Ten patients ( 7 with a skull base meningioma) planned for routine SCRT were replanned for IMSRT. The criteria for comparison were the same for both methods: optimal dose to the planning target volume (PTV) and optimal sparing of the organs at risk (OAR). For SCRT, sparing of OAR was achieved by conformal avoidance using 5-6 fields. The IMSRT inverse planning process used optimized OAR sparing through user-defined dose constraints. Dose to the PTV and OAR were assessed by dose-volume histograms, maximum dose, 2 conformity indices, and volumes of relevant isodoses.

Results: The conformity index is consistently higher for IMSRT, the largest improvement being for the multifocal and irregular cases. Volumes of the $90 \%$ and $80 \%$ isodoses were smaller for IMSRT, whereas the volume of the $30 \%$ isodose was larger for IMSRT in 6 cases. The maximum dose was consistently higher for IMSRT (mean values $102 \%$ and $108 \%$ for SCRT and IMSRT, respectively). Sparing of OAR was better with IMSRT, especially for those OARs situated in or near a concave PTV. Conclusions: In terms of PTV coverage, there is an advantage in using IMSRT for all target shapes, but especially for irregular and concave targets. The dose to OAR is lower with IMSRT, although the volume of normal tissue receiving a low dose can be larger than for SCRT.

Keywords: Stereotactic conformal radiotherapy, Micromultileaf collimator, Base-ofskull meningioma, Intensity-modulated radiotherapy, Conformity index. 


\section{INTRODUCTION}

A three-dimensional conformal treatment technique may be superior to conventional radiation therapy in the irradiation of brain tumors, because many lesions are irregularly shaped and close to critical structures. Because intensity-modulated radiotherapy (IMRT) has become clinically available, it seems logical to use it in the treatment of brain tumors or even to combine it with stereotactic conformal radiotherapy (SCRT) to yield intensity-modulated stereotactic radiotherapy (IMSRT). This is especially true for tumors situated in the skull base, such as meningiomas of the cavernous sinus and sphenoid ridge, which are located near critical cranial nerves, important vascular structures, and the brainstem. These tumors often show concavities within the planning target volume (PTV), in which organs at risk (OAR) such as the brainstem, the pituitary, or other normal tissues are located. SCRT based on multiple static fields is often suited to the treatment of convex or ovoid-shaped lesions. IMRT has been shown to have the ability to conform the dose to concavities and to better avoid critical organs ${ }^{1,2}$, although most studies have been performed for large tumors. With motorized micromultileaf collimation systems now commercially available, IMRT can be extended to smaller intracranial tumors. IMRT allows the modulation of beam intensity to achieve the desired dose distribution for the PTV and the OAR by using inverse planning strategies, optimization algorithms, and dose constraints.

Meningiomas are benign, slow-growing tumors arising from the meninges and represent $20 \%$ of all primary central nervous system tumors. They characteristically grow along cranial nerves and bony structures. The standard treatment for meningioma is a maximum safe surgical resection, which is difficult to achieve for deeply seated tumors in the base of the skull, because surgery can be associated with significant morbidity. Radiotherapy is used for residual, recurrent, or surgically inaccessible tumors, although its role is not fully proven. For meningiomas, fractionated SCRT and radiosurgery used in the treatment of residual, recurrent, or inoperable tumors have a high tumor control. Both can achieve a similar tumor control rate of $89 \%$ to $100 \%$ at 5 years $^{3-5}$, compared to conventional radiotherapy with a reported tumor control of $85 \%$ at 10 years ${ }^{6}$. The difference between those two approaches, however, is the risk of morbidity. Critical organs such as cranial nerves in the cavernous sinus or the brainstem are frequently part of the target volume and are therefore at risk of receiving high single-fraction doses ${ }^{3}$. With fractionated SCRT, the dose per fraction is below radiation tolerance levels of normal brain structures and shows less morbidity ${ }^{5}$. The next step seems then to be an extension in dose conformality, which is a feature of IMRT. 
Despite the existence of different three-dimensional treatment techniques such as SCRT and other complex approaches to radiotherapy, it is unclear if one approach is superior to another, or if certain circumstances would favor one approach or another. Recent studies reported an advantage in using IMRT for small intracranial tumors and meningiomas ${ }^{1,7-9}$, but they have not clearly defined a subgroup of tumors that would benefit most from the new technique. This study evaluates whether fractionated IMSRT based on dynamic leaf motions of a micromultileaf collimator (mMLC) can further optimize fractionated SCRT using uniform intensity beams and a static mMLC with respect to target volume conformation and avoidance of surrounding normal brain structures, thus leading to a lower risk of long-term radiation side effects. The study also aims to define a subgroup of tumors for which IMSRT might be preferable to SCRT. Hence, a selection of 10 clinical cases treated with SCRT was reconsidered for IMSRT with respect to dose conformation to the PTV and OAR. The analysis of conformality involved an additional conformity index evaluating the location of the reference isodose in relation to the position of the $\mathrm{PTV}^{10}$.

\section{METHODS AND MATERIALS}

\section{Overview}

Ten patients to be treated with SCRT were selected for the study. Of these, 7 had a skull base meningioma, 1 a falx meningioma, and 2 had other brain lesions, namely a recurrent squamous cell carcinoma of the tonsil situated in the epipharynx near the foramen magnum, and a suprasellar craniopharyngeoma. Table 1 shows a description of tumor location, prescribed SCRT dose, and the target volumes.

The volumes of the base-of-skull meningiomas were the largest volumes to be treated. The median gross tumor volume and PTV volume was 9.9 and $28.5 \mathrm{~cm}^{3}$, respectively. The gross tumor volume was expanded three dimensionally by a $3-\mathrm{mm}$ margin to form the PTV in accordance with SCRT routine treatment. All tumors were located close to an OAR; 4 tumors (Cases 1, 3, 4, 10) were mainly ovoid in shape, but with concave incursions visible in the sagittal view, whereas 5 skull base meningiomas (Cases 2, 5-8) were irregularly shaped and formed concavities (mainly around the brainstem and the pituitary). The falx meningioma was multifocal (Case 9). Table 2 shows the defined OAR and summarizes dose constraints and penalties used for intensity-modulated inverse planning. The chosen dose maxima are relative and dependent on the total dose and fractionation schedule. The particularly low dose limit for the spinal cord was set because of a previous curative radiotherapy to the area. 
Table 1. Patient characteristics and target volumes

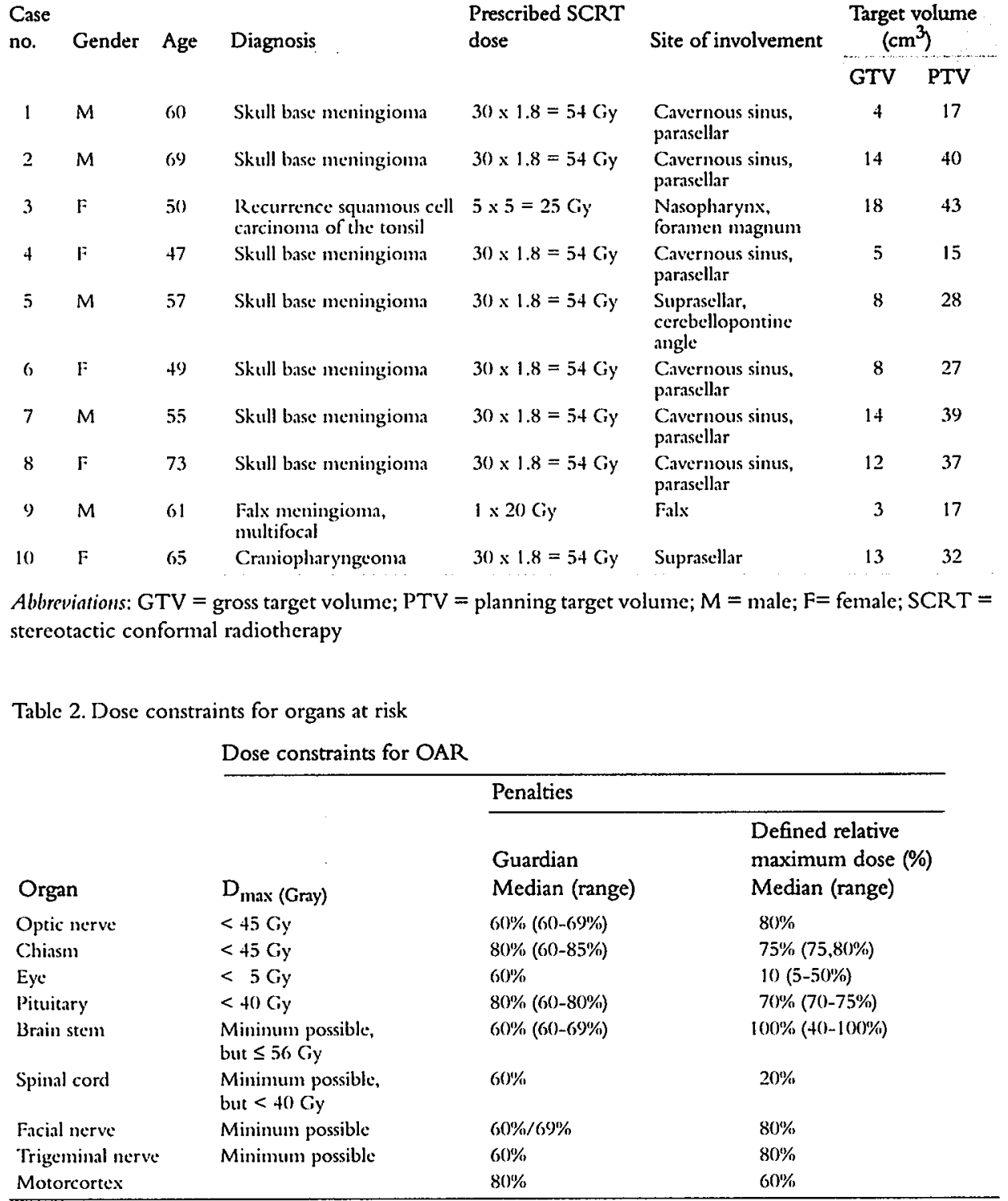

Abbreviations: $\mathrm{OAR}=$ organs at risk; $\mathrm{D}_{\text {max }}=$ maximum dose

Notes: The guardian is the relative weighting between OAR. The relative maximum dose relates to the maximum dose individually defined for each OAR. 
Patients were immobilized and scanned in a relocatable stereotactic frame (BrainLAB AG, Heimstetten, Germany). For 8 patients, a customized bite block was used. For 1 patient, a standardized upper-jaw fixation system provided by BrainLAB was used. One patient was immobilized by using a fixed rigid stereotactic frame for radiosurgery. The relocation accuracy of the BrainLAB mask system has been measured to be within 1-2 mm. This is in agreement with published data and comparable with other relocatable SCRT fixation systems ${ }^{11-14}$. CT images with i.v. contrast medium were acquired by a Somatom Plus 4 CT scanner (Siemens Medical Systems, Erlangen, Germany) from the base of the fiducial system (about $5 \mathrm{~cm}$ or more below the base of the skull) to the top of the skull. The slice thickness and distance were 3 mm.

\section{Dose calculation}

For treatment planning, $\mathrm{CT}$ and $\mathrm{MR}$ data and the Brain-LAB treatment planning system (TPS) with BrainSCAN 5.0 software were employed. For SCRT planning, the Clarkson dose calculation algorithm was used. This is a simple algorithm and accurate when no air cavities are present in the beams ${ }^{15,16}$. For IMSRT planning, a pencil beam algorithm was used. Optimization was done by using the dynamically penalized likelihood method, a variant of the maximum likelihood estimator method of statistical parameter estimation ${ }^{17,18}$. All dose distributions were normalized at the isocenter. Two different collimation systems were used for this investigation. For SCRT planning, a manually operated mMLC based on a DKFZ design (Deutsches Krebs Forschungs Zentrum Heidelberg ${ }^{19}$ ) was used. This mMLC has 2 sets of 40 nondivergent leaves with a leaf width of $1.5 \mathrm{~mm}$ at the isocenter. The $80 \%-20 \%$ penumbra width is $2 \mathrm{~mm}$ at the isocenter. The distance between the bottom of the mMLC and the X-ray source is $67.5 \mathrm{~cm}$. IMSRT planning was based on the BrainLAB M3 mMLC, which has 26 leaf pairs with variable leaf width: 14 pairs with $3 \mathrm{~mm}$ (at the isocenter), 6 pairs with $4.5 \mathrm{~mm}$, and 6 pairs with $5.5-\mathrm{mm}$ leaf width. The characteristics have been described elsewhere ${ }^{20}$. The effective $80 \%-20 \%$ penumbra width is $<3.0 \mathrm{~mm}$ for all field sizes ${ }^{20}$. The leaves move perpendicularly to the beam central axis and are nondivergent in the direction of motion, but leaf edges are rounded off and approximately correspond to the divergence of the beam. The leaves are divergent in the direction perpendicular to the direction of motion. Further characteristics are available in the literature ${ }^{20,21}$.

\section{Treatment planning}

For this dosimetric study, the same set of patients, the same number of nonconformal beams, an identical beam orientation, and nominal beam energy of $6 \mathrm{MeV}$ were used. The optimal beam orientation was defined for SCRT by geometric methods 
using the dose constraints given in Table 2. The same requirements for PTV coverage were used for SCRT and IMSRT. This was a $100 \%$ coverage of the target volume by the $95 \%$ isodose. A $95 \%$ coverage of $90 \%$ of the volume was deemed acceptable if the PTV was adjacent to an OAR.

In SCRT planning, conformation was achieved by manually conforming multiple noncoplanar static beams to the projections of the PTV in each beam's-eye-view $(\mathrm{BEV})$. Sparing of the OAR was achieved by conformal avoidance and a careful selection of the beam angles. Dose-volume histograms (DVH) were used to manually optimize dose to the PTV and OAR. Reduction of dose to the OAR was achieved mainly through geometric methods. A field setup of 5 to 6 fields was used, which corresponds to clinical treatment practice. Patients who were treated with fewer than 5 fields were replanned for purposes of comparison.

For IMSRT, inverse planning was used, based on the manually optimized SCRT beam orientations. The requirements for both PTV coverage and OAR dose constraints were the same as for the SCRT plan. Additionally, the set maximum dose was $<110 \%$. Optimization was done by adjusting weighting factors only, without changing individual constraints or beam orientations. The IMSRT planning process optimized critical organ dose sparing while maintaining PTV dose conformality. Dose constraints used for OAR in terms of absolute and relative dose and dose guardians were as defined in the BrainSCAN TPS and are shown in Table 2 and Fig. 1. Additionally, constraint limits can be placed in the DVH as shown in Fig. 1 with DVH graphs, which allow the definition of relative weighting on OAR and the PTV. Algorithm parameters such as beamlet size and grid size were also optimized during IMSRT planning. The optimization process is better when the grid size is set as small as possible. Optimizations involving small volumes (e.g., chiasm, pituitary) were adversely affected by larger calculation grid size.

\section{Comparison of treatments and analysis of conformity}

The SCRT and IMSR T plans were assessed both quantitatively and qualitatively: by visual inspection of the dose distribution and by the use of DVH and conformity index (CI). DVH were obtained for the target, the nontarget normal brain, and all organs at risk. Additional criteria for comparison are the volumes enclosed by isodoses of $90 \%, 80 \%, 50 \%$, and $30 \%(V 90 \%, V 80 \%, \sqrt{5} 0 \%$, and $V 30 \%)$.

One criterion defined by the RTOG Stereotactic Radiosurgery Committee is an assessment of the PTV coverage of a defined isodose (e.g., the $95 \%$ isodose), which is defined through the $\mathrm{CI}^{22}$. One method of determining the $\mathrm{CI}$ is the measurement of PTV coverage by one isodose volume ${ }^{20}$ : 

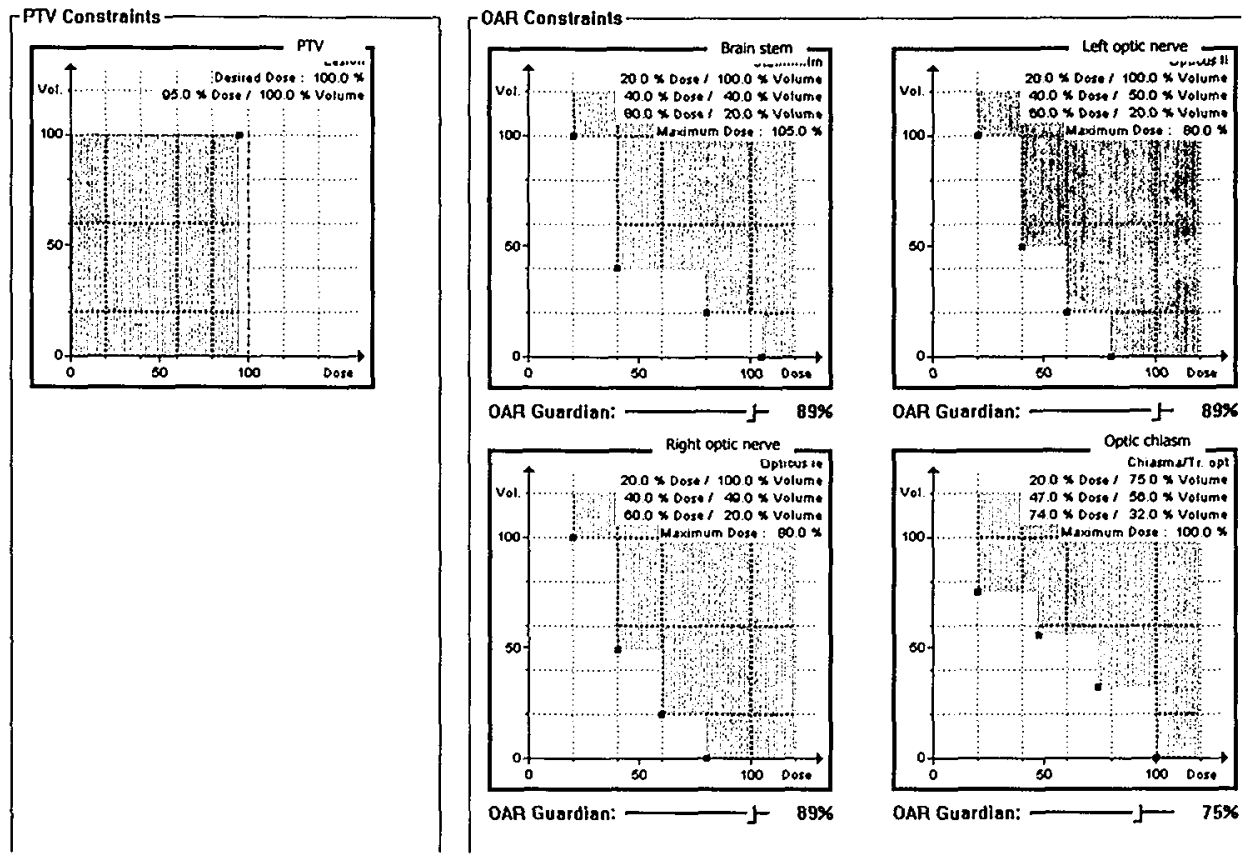

Fig. 1. The constraints for IMSRT both for the PTV and OAR are set with these DVH. The maximum dose for different partial volumes can be defined, as well as a dose guardian for relative importance weighting between OAR and a relative maximum dose (Sec Table 2). For example, for the PTV, the requirement is set for $95 \%$ coverage for $100 \%$ of the volume. For OAR, additional partial volume constraints could be defined. The shaded area represents the constraint-restricted volumes.

$$
C I_{95 \%}=\frac{P I V}{T V}
$$

Where PIV $=95 \%$ isodose volume, and TV $=$ volume of PTV.

A new $\mathrm{Cl}$ that also takes into account the location of the prescription dose volume (PIV $=95 \%$ isodose volume) relative to the target volume (TV $=$ volume of PTV) has been defined by Paddick ${ }^{10}$. This is described by the ratio of the target volume within the prescribed isodose surface, as follows:

$$
C I_{95 \%}=\frac{T V_{P I^{2}}}{T V \times P I V}
$$

Both indices measure the degree of conformity and ideally equal 1 . For this study, both indices were compared with each other as well as with results from the literature. 


\section{RESULTS}

\section{Conformality}

Comparison of isodose volumes: In general, normal-tissue sparing for the high-dose volumes and OAR was better with IMSR T. The lower isodose volumes ( $\leq V 50 \%)$, and therefore the integral dose, were higher with intensity modulation in $6 / 10$ cases. The mean $V 90 \%$ and $V 80 \%$ are smaller with IMSRT: With SCR T, the $V 90 \%$ and $V 80 \%$ were larger by $25 \%$ and $17 \%$, respectively (Table 3 ). The same holds true for the mean $V 50 \%$, but the difference is smaller ( $7 \%$ ). For the $V 30 \%$, there is practically no difference between the mean volumes. On the other hand, if specific tumor groups are considered, for example, the irregularly shaped skull base meningiomas (Cases 2, 5-8), with IMSRT less normal tissue is irradiated for all isodose volumes. For Cases 7 and 8 (Table 3), a large advantage in sparing of normal tissue within the $V 30 \%$ (up to $100 \mathrm{~cm}^{3}$ ) is seen with IMSRT, whereas for Cases 1 (ovoid) and 9 (multifocal), this advantage is only moderate. In the remaining 6 cases, the $V 30 \%$ is larger for IMSR T . A higher conformality with less normal tissue irradiated for all isodoses calculated is also seen for the multifocal meningioma (Case 9). The isodose distributions in the PTV may be described as homogeneous for both planning methods. High-dose volumes observed in IMSRT do not have excessive dose maxima and are small (Fig. 2, Table 3). The median maximum dose is slightly higher for IMSRT with $108 \%$ compared to $102 \%$ for SCRT.

Conformality to PTV: For this study we compared the two different CI defined above. The index from the RTOG (referred to here as the "old index") 22 and the index from Paddick (referred to here as the "new index") ${ }^{10}$, which additionally takes into account the localization of the PTV relative to the $95 \%$ isodose, were chosen. An ideal conformation was not achieved by either technique. In Table 4, it can be seen that the new CI shows an improvement in conformality for IMSRT over SCRT for all cases, whereas the old CI detected this advantage for only 6 patients. For Cases 4,7 , 8 , and 9 , the old CI showed no improvement in using IMSRT. These findings translate into an equal mean $\mathrm{CI}_{95 \%}$ for the old $\mathrm{CI}$ of 1.01 for both techniques. In contrast, the new $\mathrm{CI}$ shows an 18 percentage point improvement in the mean conformality for IMSRT (SCRT CI $95 \%$ : 0.65, IMSRT CI ${ }_{95 \%}$ : 0.83). Overall, the $\mathrm{CI}_{95 \%}$ is always better when using intensity modulation. The largest improvement (37 percentage points) is seen for Case 9 (multifocal) with a new $\mathrm{CI}_{95 \%}$ of 0.78 for IMSRT and 0.41 for SCRT, but all irregular tumors receive a more conformal dose with IMSRT. The aim to have at least $90 \%$ of the volume within the $95 \%$ isodose was not always possible, because of conflicts resulting from the position of OAR relative to the PTV rather than from dose constraints used for OAR. 
Table 3. Isodose volumes and Maximum Dose

\begin{tabular}{|c|c|c|c|c|c|c|c|c|c|c|}
\hline \multirow[b]{2}{*}{ Case } & \multicolumn{2}{|c|}{ Volume $\left(\mathrm{cm}^{3}\right) 90 \%$} & \multicolumn{2}{|c|}{ Volume $\left(\mathrm{cm}^{3}\right) 80 \%$} & \multicolumn{2}{|c|}{ Volume $\left(\mathrm{cm}^{3}\right) 50 \%$} & \multicolumn{2}{|c|}{ Volume $\left(\mathrm{cm}^{3}\right) 30 \%$} & \multicolumn{2}{|c|}{ Dose $_{\max }$} \\
\hline & SCRT & IMSRT & SCRT & IMSRT & SCRT & IMSRT & SCRT & IMSRT & SCRT & IMSRT \\
\hline 1 & 22.1 & 21.2 & 27.6 & 24.9 & 46.2 & 57.4 & 202.5 & 189.3 & 101 & 109 \\
\hline 2 & 49.8 & 47.2 & 63.6 & 59.4 & 137.6 & 138.7 & 281.0 & 365.8 & 105 & 109 \\
\hline 3 & 57.5 & 50.4 & 73.5 & 60.7 & 142.8 & 125.2 & 316.4 & 359.6 & 103 & 107 \\
\hline 4 & 21.0 & 16.6 & 25.9 & 20.1 & 48.0 & 48.1 & 110.4 & 129.7 & 102 & 109 \\
\hline 5 & 37.6 & 33.7 & 47.5 & 41.1 & 89.9 & 86.4 & 233.3 & 262.3 & 102 & 108 \\
\hline 6 & 37.0 & 33.6 & 46.9 & 39.7 & 83.1 & 81.6 & 234.2 & 259.8 & 103 & 107 \\
\hline 7 & 58.0 & 42.3 & 72.3 & 53.5 & 144.6 & 125.1 & 443.4 & 342.2 & 102 & 108 \\
\hline 8 & 59.1 & +2.8 & 73.3 & 52.5 & 136.2 & 110.1 & 403.1 & 303.0 & 100 & 107 \\
\hline 9 & 28.2 & 22.2 & 38.3 & 28.8 & 71.0 & 58.3 & 144.2 & 133.0 & 105 & 107 \\
\hline 10 & 38.2 & 36.7 & 46.5 & 42.8 & 81.7 & 78.2 & 213.1 & 278.5 & 102 & 104 \\
\hline Mcan & 40.9 & 30.4 & 51.5 & 42.5 & 98.2 & 91.1 & 258.2 & 262.7 & 102 & 108 \\
\hline
\end{tabular}

Abbreviations: Dose $_{\mathrm{mux}}=$ maximum dose; $\mathrm{SCRT}=$ stereotactic conformal radiotherapy; $\mathrm{IMSRT}=$ intensity modulated stereotactic radiotherapy.

Table 4. Conformity indices

\begin{tabular}{|c|c|c|c|c|}
\hline \multirow[b]{3}{*}{ Case } & \multicolumn{4}{|l|}{$\mathrm{CI}_{95 \%}$} \\
\hline & \multicolumn{2}{|l|}{ SCRT } & \multicolumn{2}{|l|}{ IMSRT } \\
\hline & Old (RTOG) & New (Paddick) & Old (RTOG) & New (Paddick) \\
\hline 1 & 0.87 & 0.68 & 1.06 & 0.82 \\
\hline 2 & 0.92 & 0.67 & 0.95 & 0.80 \\
\hline 3 & 0.97 & 0.65 & 1.02 & 0.88 \\
\hline 4 & 1.01 & 0.68 & 0.89 & 0.82 \\
\hline 5 & 0.96 & 0.68 & 1.03 & 0.83 \\
\hline 6 & 1.05 & 0.71 & 1.08 & 0.84 \\
\hline 7 & 1.15 & 0.66 & 0.88 & 0.82 \\
\hline 8 & 1.21 & 0.67 & 1.01 & 0.84 \\
\hline 9 & 1.11 & 0.41 & 1.08 & 0.78 \\
\hline 10 & 0.89 & 0.70 & 1.05 & 0.90 \\
\hline Mean & 1.01 & 0.65 & 1.01 & 0.83 \\
\hline
\end{tabular}

Abbreviations: $\mathrm{CI}_{{ }_{55} \%}$ : Conformity Index of $95 \%$ isodose volume; SCRT $=$ stereotactic conformal radiotherapy; IMSRT = intensity modulated stereotactic radiotherapy.

CI definitions: Old (RTOG, 22): $\mathrm{CI}_{25}=\mathrm{PIV} / \mathrm{TV}$ (PIV $=$ prescription isodose volume; $\mathrm{TV}=$ Volume of PTV); New (Paddick, 10): $\mathrm{Cl}_{95}=\mathrm{TV}_{\mathrm{piv}}^{2} / \mathrm{TV} \star \mathrm{PIV}$

\section{Number of fields}

For all cases except one, the same number of fields and the same field setup were used for both techniques. Case 2 involved a large and irregular volume. SCR T planning of this volume included 2 additional smaller boost fields to the original 5 fields. Intensity modulation achieved a better conformality with 5 fields and 1 isocenter alone. For this study, the multifocal case (No. 9) was planned with 6 fixed fields. However, the patient was treated with a radiosurgery using multiple isocenters with overlapping isodoses, because IMSRT was not clinically available at the time of the study. 


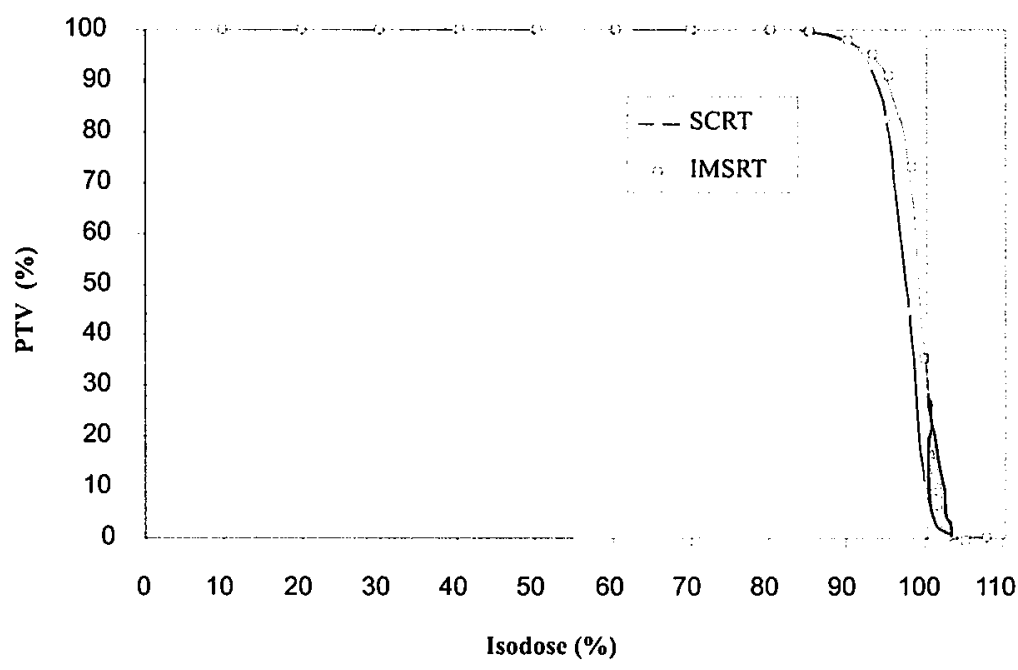

Fig. 2. Dose-volume histogram for the mean SCRT and IMSRT target volumes. The shaded area between the curves represents the difference in the mean PTV that is receiving a higher dose with IMSRT than with SCRT.

Table 5. Comparison of Dose-Volume Histograms for SCRT and IMSRT: Optic pathways

\begin{tabular}{|c|c|c|c|c|c|c|c|}
\hline \multirow{3}{*}{$\begin{array}{l}\text { Case } \\
\text { no. }\end{array}$} & \multirow{3}{*}{$\begin{array}{l}\% \text { of } \\
\text { PTV dose }\end{array}$} & \multicolumn{4}{|c|}{ Optic nerves } & & \\
\hline & & \multicolumn{2}{|l|}{$\overline{\text { left }}$} & \multicolumn{2}{|c|}{ right } & \multicolumn{2}{|c|}{ Chiasm } \\
\hline & & 80 & 30 & 80 & 30 & 80 & 30 \\
\hline \multirow[t]{2}{*}{1} & SCRT & 8.6 & 30 & 0 & 0 & 0 & 68.3 \\
\hline & IMSIRT & 5.7 & 31.4 & 0 & 0 & 0 & 56.1 \\
\hline \multirow[t]{2}{*}{2} & SCRT & 0 & 0 & 6.0 & 0.22 & 50.0 & 88.6 \\
\hline & IMSIRT & 0 & 18.4 & 12.0 & 26.0 & 13.6 & 72.7 \\
\hline \multirow[t]{2}{*}{3} & SCRT & 0 & 0 & 0 & 0 & - & - \\
\hline & IMSIRT & 0 & 7.4 & 0 & 0 & - & - \\
\hline \multirow[t]{2}{*}{4} & SCRT & 14.9 & 32.4 & 0 & 17.8 & 51.6 & 96.8 \\
\hline & IMSRT & 13.5 & 29.7 & 0) & 3.6 & 54.8 & 83.9 \\
\hline \multirow[t]{2}{*}{5} & SCRT & 0 & 1.8 & 5.0 & 15.0 & 22.9 & 100 \\
\hline & IMSRT & 0 & 3.6 & 0) & 15.0 & 5.7 & 42.9 \\
\hline \multirow[t]{2}{*}{6} & SCRT & 0 & 0 & 29.7 & 50.0 & 75.6 & 100 \\
\hline & IMSRT & 0 & 59.5 & 28.1 & 43.8 & 56.1 & 100 \\
\hline \multirow[t]{2}{*}{7} & SCRT & 22.6 & 59.7 & 0 & 16.1 & 12.9 & 96.8 \\
\hline & IMSRT & 25.8 & 53.2 & 0 & 9.5 & 0 & 58.1 \\
\hline \multirow[t]{2}{*}{8} & SCRT & 0 & 0 & - & - & 35.2 & 88.0 \\
\hline & IMSRT & 0 & 0 & - & - & 24.1 & 82.4 \\
\hline \multirow[t]{2}{*}{10} & SCRT & 22.9 & 62.9 & 22.7 & 56.8 & 90.2 & 100 \\
\hline & IMSRT & 22.9 & 62.9 & 22.7 & 59.1 & 53.7 & $100)$ \\
\hline
\end{tabular}

Abbreviations: PTV = Planning target volume; SCRT $=$ stereotactic conformal radiotherapy, $\operatorname{IMSRT}=$ intensity modulated stereotactic radiotherapy 
Table 6. Comparison of Dose-Volume Histograms for SCRT and IMSRT: Critical structures situated in concavitics of the tumor

\begin{tabular}{|c|c|c|c|c|c|}
\hline \multirow[b]{2}{*}{ Case no. } & \multirow[b]{2}{*}{ \% PTV dose } & \multicolumn{2}{|c|}{ Pituitary } & \multicolumn{2}{|c|}{ Brain stem } \\
\hline & & 80 & 30 & 80 & 30 \\
\hline \multirow[t]{2}{*}{1} & SCRT & 73.1 & 100 & 0.3 & 23.3 \\
\hline & IMSRT' & 19.2 & 96.1 & 0.5 & 23.4 \\
\hline \multirow[t]{2}{*}{2} & SCRT & 80.52 & 100 & 7.4 & 28.6 \\
\hline & IMSRT & +7.6 & 93.9 & 3.6 & 29.6 \\
\hline \multirow[t]{2}{*}{3} & SCR'T & - & - & 0 & 1.3 \\
\hline & IMSR'T & - & - & 0 & 0.3 \\
\hline \multirow[t]{2}{*}{4} & SCRT & 100 & 100 & 5.7 & 25.2 \\
\hline & IMSRT & 87.5 & 100 & 4.0 & 26.3 \\
\hline \multirow[t]{2}{*}{5} & SCRT & - & - & 11.6 & 33.2 \\
\hline & IMSRT & - & - & 10.9 & 33.5 \\
\hline \multirow[t]{2}{*}{6} & SCIRT & 97.1 & 100 & 9.9 & 59.1 \\
\hline & IMSRT & 81.2 & 100 & 4.7 & 38.6 \\
\hline \multirow[t]{2}{*}{7} & SCRT & 100 & 100 & 24.1 & 79.6 \\
\hline & IMSRT & 95.2 & 100 & 8.3 & 41.0 \\
\hline \multirow[t]{2}{*}{8} & SCRT & 96.7 & 100 & 18.9 & 73.8 \\
\hline & IMSRT T & 75 & 100 & 8.7 & 38.7 \\
\hline \multirow[t]{2}{*}{10} & SCRT & - & - & 3.6 & 16.9 \\
\hline & IMSRT & - & - & 3.7 & 29.3 \\
\hline
\end{tabular}

Abbreviations: PTV $=$ Planning target volumc; $\mathrm{SCRT}=$ stercotactic conformal radiotherapy, $\mathrm{IMSRT}=$ intensity modulated

Table 7. Comparison of Dose-Volume Histograms SCRT and IMSRT: Brain nerves, motorstrip

\begin{tabular}{|c|c|c|c|c|c|c|c|}
\hline \multirow[b]{2}{*}{ Case no. } & \multirow[b]{2}{*}{$\%$ PTV dose } & \multicolumn{2}{|c|}{ Facial nerve } & \multicolumn{2}{|c|}{ Trigeminal nerve } & \multicolumn{2}{|c|}{ Motorcortex } \\
\hline & & 80 & 30 & 80 & 30 & 80 & 30 \\
\hline \multirow[t]{2}{*}{1} & SCRT & 75.0 & 100 & 100 & 100 & - & - \\
\hline & IMSIRT & 50.0 & 100 & 100 & 100 & - & - \\
\hline \multirow[t]{2}{*}{2} & SCRT & 100 & 100 & 100 & 100 & - & - \\
\hline & IMSIRT & 90.0 & 100 & 100 & 100 & - & - \\
\hline \multirow[t]{2}{*}{3} & SCRT & - & - & - & - & - & - \\
\hline & IMSRT & - & - & - & - & - & - \\
\hline \multirow[t]{2}{*}{4} & SCRT & - & - & - & - & - & - \\
\hline & IMSIRT & - & - & - & - & - & - \\
\hline \multirow[t]{2}{*}{5} & SCRT & 0 & 66.7 & - & - & - & - \\
\hline & IMSIRT & 0 & 26.7 & - & - & - & - \\
\hline \multirow[t]{2}{*}{6} & SCRT & - & - & - & - & - & - \\
\hline & IMSIRT & - & - & - & - & - & - \\
\hline \multirow[t]{2}{*}{7} & SCRT & 81.8 & 100 & - & - & - & - \\
\hline & IMSRT & 54.5 & 100 & - & - & - & - \\
\hline \multirow[t]{2}{*}{8} & SCRT & - & - & - & - & - & - \\
\hline & IMSIRT & - & - & - & - & - & - \\
\hline \multirow[t]{2}{*}{9} & SCIRT & - & - & - & - & 17.2 & 57.3 \\
\hline & IMSRT & - & - & - & - & 11.9 & 40.4 \\
\hline \multirow[t]{2}{*}{10} & SCRT & - & - & - & - & - & - \\
\hline & IMSIRT & - & - & - & - & - & - \\
\hline
\end{tabular}

Abbreviations: $\mathrm{PTV}=$ Planning target volume; $\mathrm{SCRT}=$ stcrcoractic conformal radiotherapy, IMSRT $=$ intensity modulated 


\section{Organs at risk (OAR)}

DVH: For Patient 3, IMSRT spared the brainstem best, but the dose to the optic nerves is higher than for SCRT $(7.4 \%$ vs. $0 \%$, Table 5$)$. This suggests that IMSRT achieved a lower dose to the brainstem at the expense of dose to the optic nerve (Table 6). The same holds for Cases 2 and 6: Dose is brought partially to the contralateral optic nerve ( $18.4 \%$ and $59.5 \%$ vs. $0 \%$ with SCRT, Table 5$)$. Additionally for Case 2, the ipsilateral optic nerve received $50 \%-92 \%$ more dose both in $V 80 \%$ and $V 30 \%$ using IMSR T (from $6 \%$ to $12 \%$ and $0.22 \%$ to $26 \%$, Table 5). Table 6 shows a better sparing of nearly all OAR lying in the concavities of the tumor, such as brainstem and pituitary, both for $V 80 \%$ and $V 30 \%$. This is more pronounced for the brainstem, but is also true for the other OAR. The central brain nerves and the facial and trigeminal nerves, as well as the motor cortex, are better spared with IMSRT, at least for the part of the nerve lying outside of the PTV (Table 7).

Qualitative description of case examples: Figure 3 shows an example of good sparing of the pituitary with IMSRT while maintaining the prescribed dose to the PTV at the same time. The total dose to the brainstem is lower with IMSRT. Another OAR lying in a concavity of the tumor is the brainstem. In Fig. 4, a better sparing of OAR with IMSRT compared to SCRT is observed: The dose conforms precisely to the brainstem with a slight underdosage of the PTV. An excellent dose shaping based on IMSRT planning is seen with the multifocal case in Fig. 5: The motor strip is better spared, and the normal brain tissue receives less high dose $(70 \%-95 \%$ isodose volumes), as best demonstrated on the sagittal view.

On the other hand, a smaller and ovoid-shaped tumor is better treated with SCRT (Fig. 6), because conformality of the $95 \%$ isodose was shown to be equal with both methods. Using IMSRT planning, the lower-dose areas (30\% isodose, blue dose painting) are larger, and some of the dose is redistributed to the contralateral optic nerve. With SCRT, the contralateral optic nerve receives no dose. 


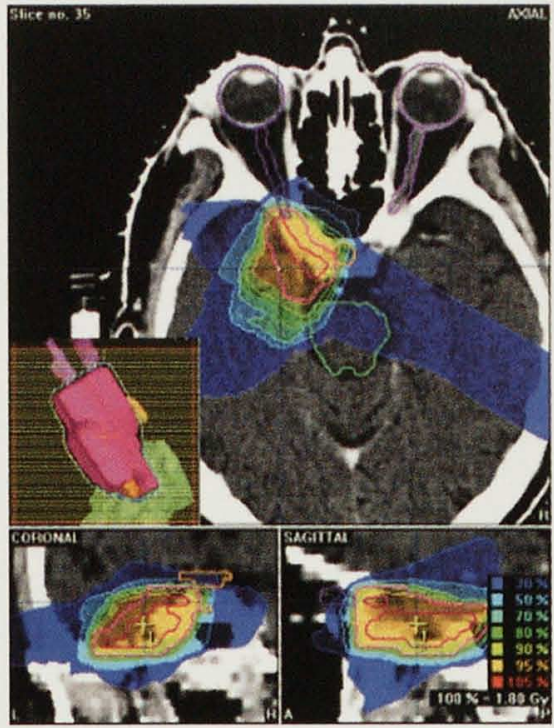

SCR T
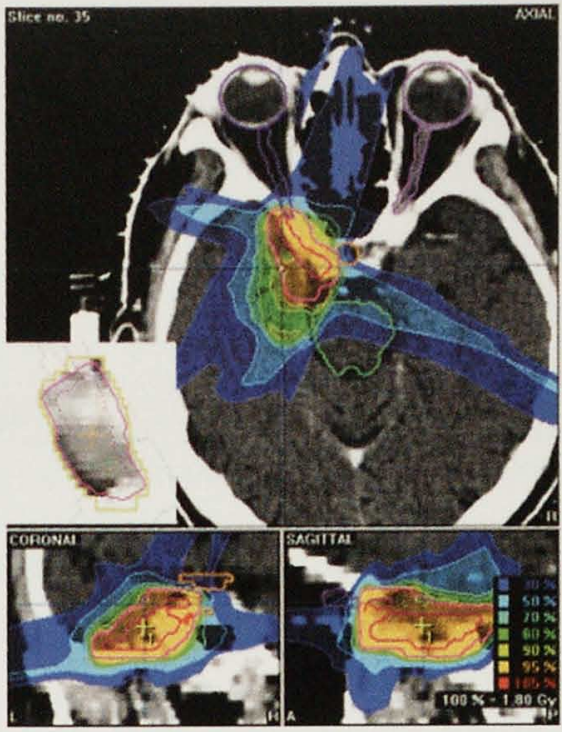

IMSRT

Fig. 3. Example of sparing the pituitary and partial sparing of the ipsilateral optic nerve in a patient with a skull base meningioma (Case 1). Isodose color wash and isodose lines are defined in the right-hand corner. An intensity map of one field is shown for IMSRT, whereas a BEV is shown for the matching field for SCRT.

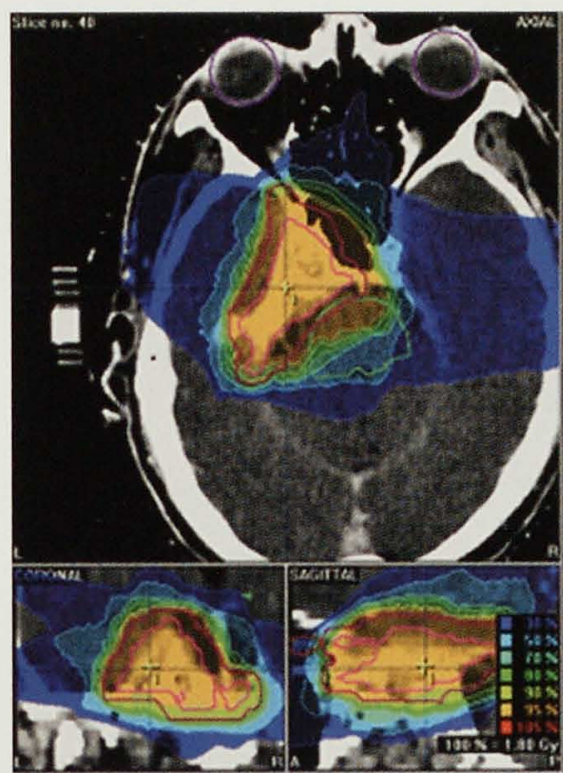

SCRT

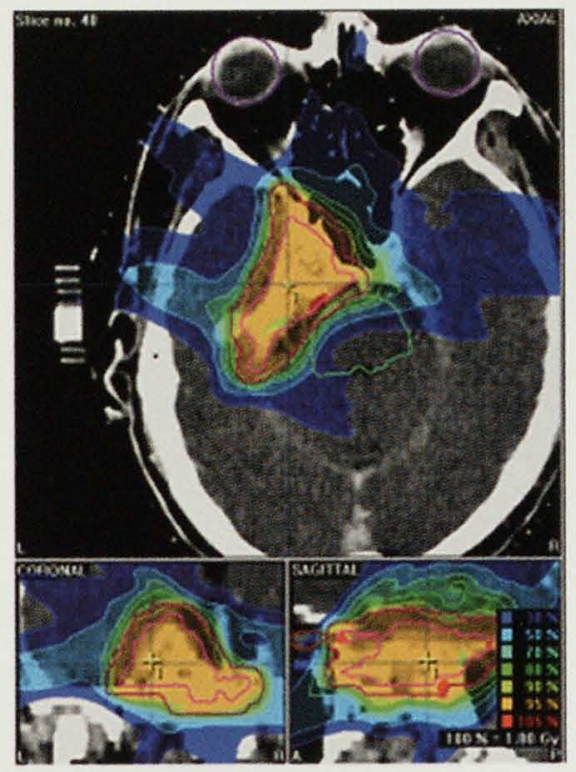

IMSRT

Fig. 4. Example of sparing of the brainstem in a patient with an irregular skull base meningioma (Case 7). With IMSRT, less brainstem volume is irradiated to a higher dose. Isodose color wash and isodose lines are defined in the right-hand corner. 


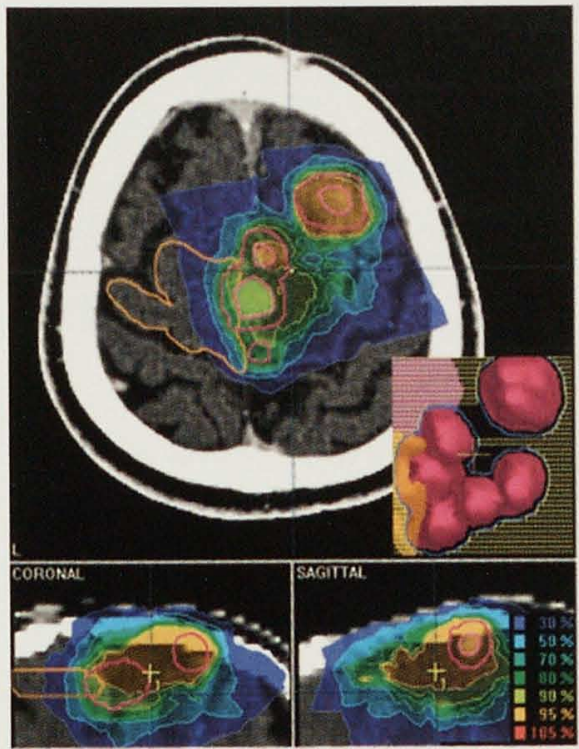

SCRT

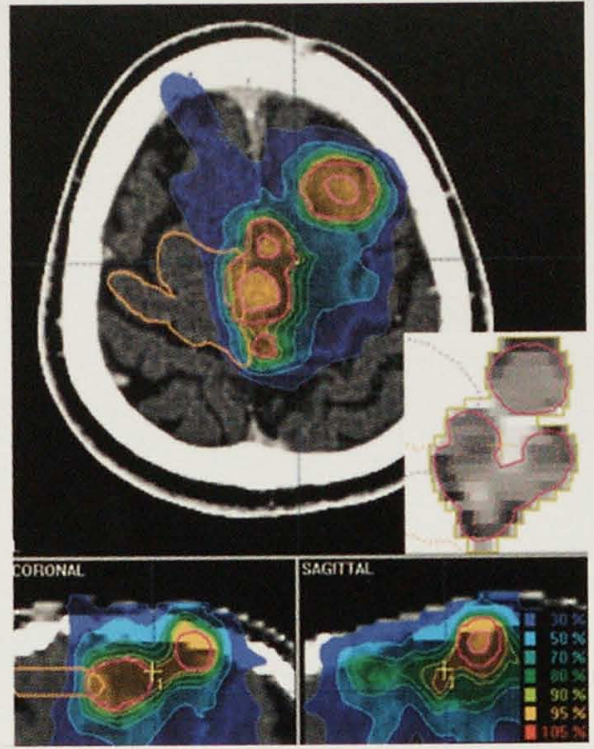

IMSRT

Fig. 5. Example of a multifocal meningioma (Case 9). Note the reduced area of the $95 \%$ isodose in the form of an "hourglass" between two recurrent tumors in the sagittal view of the IMSRT plan. Isodose color wash and isodose lines are defined in the right-hand corner. An intensity map of one field is shown for IMSRT, whereas a BEV is shown for the matching field for SCRT.

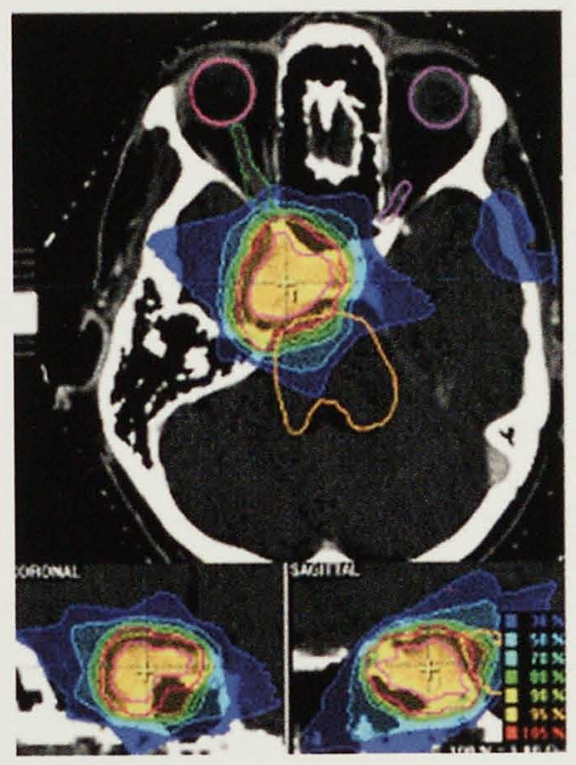

SCRT

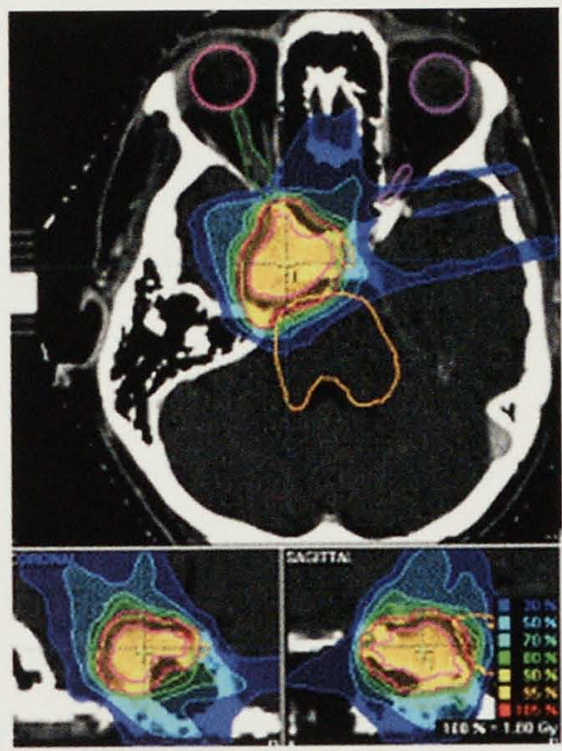

IMSR T

Fig, 6. Example of an ovoid skull base meningioma (Case 4). The contralateral optic nerve receives a dose in the IMSRT plan only. Isodose color wash and isodose lines are defined in the right-hand corner. 


\section{DISCUSSION}

For stereotactic radiotherapy, it has been demonstrated in numerous reports that treating irregular tumors with fixed fields is superior to treatment with arcs using circular collimators ${ }^{23-30}$. Taking this issue further, it may be hypothesized that the addition of intensity modulation to a fixed field arrangement should result in a further improvement for the treatment of nonspherical brain lesions. The first publications seem to prove this for irregular tumors $1,8,31,32$, whereas for spherical medium-sized tumors, no advantage or only a very modest one was seen ${ }^{9,33,34}$. The latter results, however, are dependent on the stereotactic treatment and IMRT planning methods, the comparison being based on multi-isocenter linac or gamma knife plans vs. single isocenter fixed fields, and mMLC planning vs. tomotherapy. Additionally, a comparison based on only $1-4$ cases is insufficient for drawing definite conclusions $1,8,31,33$. Furthermore, it seems to us that the use of up to 15 fields or arcs is not a very practical treatment option ${ }^{1,34}$.

This study was performed to examine the question of whether there is a benefit in using IMSRT over the SCRT technique for the fractionated treatment of tumors with an irregular shape and concavities, which often harbor OAR such as the brainstem or the pituitary. The same planning method and setup as used in daily practice was considered: All patients were planned with a 5-field setup with an optimized beam arrangement. This already resulted in a high conformality with the mean $\mathrm{CI}$ for SCRT and IMSRT being equal. The same number of fields is determined if a stochastic method (e.g., an optimization algorithm of the genetic algorithm class ${ }^{32}$ ) is applied as the optimization method: 5 fields optimized by BEV showed only small differences compared to the same setup for IMSRT in terms of $\mathrm{CI}^{32}$. This is further proof that a number of $4-6$ hand-selected fields preserves the same quality of treatment as established in earlier literature for IMSRT $T^{2,35-37}$. Seven to 15 fields or arcs as proposed by others are, therefore, not necessary ${ }^{1,38}$.

According to our routine protocol, a 3-mm margin is added three-dimensionally to allow for patient and target motion during daily treatment. For IMSRT, sufficient margins must be allowed between the edges of the target and the edges of the high-dose region: Smaller margins should be avoided, because the minimum dose to the target can drop rapidly as a result of the steep dose gradient typical for intensity modulation ${ }^{2}$ and SCRT. Regarding the amount of normal tissue irradiated, $190 \%$ and $V 80 \%$ were chosen, because they can be said to be representative of the volume treated to a significant dose. The advantage demonstrated for IMSRT over SCRT for these two volumes may be partly the result of differences in collimation. For IMSRT, the multileaf collimator is focused and has rounded edges; for SCRT, the leaves are straight edged and nonfocused. The $V 50 \%$ and $V 30 \%$ were chosen as being 
representative of the volume of normal tissue treated to a dose that may be considered at risk for late side effects. With IMSRT, less normal tissue was irradiated in the high-dose volumes. These were consistently smaller for IMSRT (mean difference $10 \%)$. Apart from large $\left(30-40 \mathrm{~cm}^{3}\right)$ and irregular skull base meningiomas, 1 ovoid, and the multifocal case, the amount of normal tissue within a low dose $(V 30 \%)$ was always larger with IMSRT. Using the same treatment planning system, Benedict et al. ${ }^{1}$ observed similar results: In 3 of 4 cases, IMSRT generally yielded a smaller volume of irradiated tissue, defined here as doses greater than $50 \%$ of the prescription dose with differences ranging from $5 \%$ to $18 \%$. Similar results are reported by two other groups, which found larger $V 10 \%-V 50 \%$ for IMSR T compared to SCRT and gamma knife plans ${ }^{32,} 39$. Homogeneity was preserved throughout both planning methods: No excessive dose maxima were seen using a single-isocenter technique, and the mean was only slightly higher for IMSRT (108\% vs. $102 \%$ for SCRT; see Table 3).

We have observed that a significant reduction in dose to OAR can be reached with intensity-modulated planning and $\mathrm{mMLC}$, especially for tumor shapes harboring the brainstem or the pituitary within concavities of the PTV, which is often found in skull base meningiomas. We could actually show that the brainstem and pituitary were better spared with IMSR T for high- and low-dose volumes ( $V 80 \%$ and $V 30 \%$ ). However, in 3 cases, this was achieved through a dose redistribution that is in the nature of the IMRT approach. The central brain nerves and the motor cortex were also better spared with IMSRT, whereas for ovoid tumors, the sparing of OAR was not better, with $V 30 \%$ being equal or larger with intensity modulation. In such a study, the conclusions drawn are to some extent dependent on the technique and algorithm (optimization process) used, and the results may therefore be different. Similar results of greater sparing of adjacent OAR were reported by the two authors using the same BrainLAB TPS ${ }^{1,8}$. Leavitt et al. ${ }^{8}$ looked at an irregular skull base meningioma, but concentrated on the optic nerves. No data for organs within concavities such as the brainstem were given. When using the Peacock System (NOMOS Corporation), for instance, dose to the risk organs may be higher ${ }^{9,34}$.

Other parameters influencing the outcome of a plan are technical parameters such as penumbra characteristics, calculation grid size, or planning parameters such as dose constraints. In this study, we have kept these parameters constant as far as possible, but a small bias remains, because of the experience of the planner for SCRT and because of differences in the dose calculation algorithms used. For IMSRT, the redistribution of dose depends on weighting factors and dose constraints used. Certain beam paths have low weights, yet dose must be delivered to the target. The price is higher weight beam paths elsewhere with higher peripheral doses along those paths. Dose redistribution could be different if different beam angles or a higher number of beams were 
used. The latter might eventually increase the integral dose. Another aspect is the position of the tumor relative to the OAR: Especially in the skull base, the tumor is surrounded by several different OARs, resulting in multiple different dose constraints.

For this study we compared two different conformality indices: the index from the RTOG ("old index"22) and the index from Paddick ("new index"10). The latter is more descriptive, because it additionally takes into account the localization of the PTV in relation to the $95 \%$ isodose. When using the old CI, fewer improvements in conformality for IMSRT were observed. In contrast, when using the new CI, improvement in each patient was observed, resulting in an improvement of 18 percentage points in the mean conformality for IMSRT. The apparent high scoring of the old CI may suggest an overtreatment, because the index was mostly higher than 1.00. The new index gives therefore a more objective assessment with its ideal value and maximum being 1.00. Because the new $\mathrm{CI}$ is not in general use yet, we used the old $\mathrm{CI}$ for comparison with data from the literature. The largest improvement was seen for the case with the multifocal tumor and all irregular tumors with IMSRT (See Table 4). Several authors have reported a general improvement in conformality, with differences of ca. $20 \%{ }^{31-33}$ and $10 \%{ }^{9}$ in favor of the IMSRT plans. Woo et al. ${ }^{33}$ describe an advantage for IMSRT for large, irregular volumes and multifocal tumors, albeit for the latter an equal and not a higher conformity than for SCRT. The reason could lie in the different IMRT system used, because radiosurgery was compared with tomotherapy.

Normal-tissue sparing for the high-dose volumes and OAR was excellent with IMSRT, especially for larger irregular volumes. However, the lower isodose volumes and, consequently, the integral dose were higher with intensity modulation in $60 \%$ of the cases. The dose reduction to some areas of the treated volume results in a possible dose increase in another area. As long as the dose constraints used are below the known threshold limits for late complications, this is acceptable. Normal tissue complication probability calculations comparing radiosurgery and IMSRT were comparable for both treatment modalities, because current models of tissue tolerance consider the larger low-dose volumes to be clinically insignificant ${ }^{34}$. However, these models do not necessarily provide accurate values for complications. Within a group of 40 patients with intracranial meningiomas treated with IMRT using tomotherapy, one possible treatment-related death is reported. The authors scored this as late radiation-related toxicity due to a small brainstem necrosis that was pathologically verified ${ }^{7}$. This necrotic volume $(4.74 \mathrm{cc})$ was found to be in the high-dose part (exceeding the maximum dose constraint of $55.6 \mathrm{~Gy}$ ) of the brainstem volume. The maximal dose constraint to the brainstem was $54 \mathrm{~Gy}$. Two patients progressed; the other 37 patients with a skull base meningioma were progression free at the time 
of reporting. Dose inhomogeneities and resulting high maximum doses may also be used for the purpose of dose escalation. This was reported recently for a group of skull base meningiomas treated with fractionated IMRT, where dose maxima up to 61$66 \mathrm{~Gy}$ were observed for $57.6 \mathrm{~Gy}$ prescribed dose. The resulting increased dose and increased dose inhomogeneity was thought to be the reason for observed early treatment responses with tumor shrinkage, which is not seen with conventional fractionated radiotherapy ${ }^{40}$. This was not combined with a higher rate of complication; however, this study had a short median follow-up of 36 months.

In conclusion, we can say the following for the cases studied:

1. Using an optimized field setup based on BEV, SCRT can result in high conformality.

2. There is an advantage in using IMSRT for all target shapes with a better conformality for all tumor forms studied.

3. We could identify a subgroup of tumor shapes that would most benefit from IMSRT in terms of conformality, reduced volume of normal tissue treated to a low dose, and sparing of neighboring OAR: For irregular and concave targets (as skull base meningioma), IMSRT is clearly better than conventional SCRT.

4. For spherical and ovoid-shaped volumes, there is only a marginal advantage in using IMSRT instead of SCRT.

5. Dose to OARs is often reduced with IMSRT, especially for those OARs situated within concavities of the PTV (as, e.g., the brainstem).

6. The use of IMSRT results in a higher integral dose in less than two-thirds of the cases.

\section{REFERENCES}

1. Benedict $\mathrm{SH}, \mathrm{Cardinale} \mathrm{RM}, \mathrm{Wu} \mathrm{Q}$, ct al. Intensity-modulated stereotactic radiosurgery using dynamic micro-multileaf collimation. Int J Radiat Oncol Biol Phys 2001;50(1):751-758.

2. Verhey LJ. Comparison of three-dimensional conformal radiation therapy and intensity-modulated radiation therapy systems. Semin Radiat Oncol 1999;9:78-98.

3. Hakim R, Alexander E 3rd, Loeffler JS, et al. Results of linear accelerator-based radiosurgery for intracranial meningiomas. Neurosurgery 1998;42(3):446-453.

4. Kondziolka D, Flickinger JC, Perez B. Judicious resection and/or radiosurgery for parasagittal meningiomas: Outcomes from a multicenter review. Gamma Knife Meningioma Study Group. Neurosurgery 1998;43(3):405-413.

5. Jalali R, Loughrey $\mathrm{C}$, Baumert $\mathrm{B}$, et al. High precision focussed irradiation in the form of fractionated stereotactic conformal radiotherapy (SCRT) for benign meningiomas predominantly in the skull base location. Clin Oncol (R Coll Radiol) 2002;14(2):103-109. 
6. Nutting C, Brada M, Brazil L, ct al. Radiotherapy in the treatment of benign meningioma of the skull base. J Neurosury 1999;90(5):823-827.

7. Uy NW, Woo SY, The BS, et al. Intensity-modulated radiation therapy (IMRT) for meningioma. Int J Radiat Oncol Biol Phys 2002;53:1265-1270.

8. Leavitt DD, Watson $\mathrm{G}$, Tobler $\mathrm{M}$, et al. Intensity-modulated radiosurgery/radiotherapy using a micromultileaf collimator. Int J Radiat Oncol Biol Phys 2001;26:143-150.

9. Khoo VS, Oldham M, Adams EJ, et al. Comparison of intensity-modulated tomotherapy with stereotactically guided conformal radiotherapy for brain tumors. Int J Radiat Oncol Biol Phys 1999;45(2):415-425.

10. Paddick I. A simple scoring ratio to index the conformity of radiosurgical treatment plans. $J$ Neurosurg 2000;93(Suppl. 3):219-222.

11. Hodapp N, Nanko N, Rohner F, et al. Quality assurance for non-invasive patient fixation during stereotactic convergent beam irradiation. Acto Neurochir Suppl (Wicn) 1994;62: 101-104.

12. Willner J, Flentje M, Bratengeier K. CT simulation in stereotactic brain radiotherapy-analysis of isocenter reproducibility with mask fixation. Radiother Oncol 1997;45(1):83-88.

13. Warrington AP, Laing RW, Brada M. Quality assurance in fractionated stereotactic radiotherapy. Radiother Oncol 1994;30:239-246.

14. Alheit $H$, Dornfeld $S$, Dawel $M$, ct al. Patient position reproducibility in fractionated stereotactically guided conformal radiotherapy using the BrainLab Mask system. StrahlentherOnkol 2001;5:264-268.

15. Clarkson JR. A note on depth dose in fields of irregular shape. BrJRadiol 1941;14:155-159.

16. Linthout N, Verellen D, Van Acker S, ct al. Evaluation of dose calculation algorithms for dynamic arc treatments of head and neck tumors. Radiother Oncol 2002;64:85-95.

17. Llacer J, Solberg TD, Promberger C. Comparative behavior of the dynamically penalized algorithm in inverse radiation therapy planning. Phys Med Biol 2001;46(10):2637-2663.

18. Llacer J. Inverse radiation treatment planning using the Dynamically Penalized Likelihood method. Med Phys 1997;24(11):1751-1764.

19. Schlegel W, Pastyr $O$, Bortfeld $T h, e t$ al. Stereotactically guided fractionated radiotherapy: Technical aspects. Radiother Oncol 1993;29:197-204.

20. Cosgrove VP, Jahn U, Pfaender M, et al. Commissioning of a micro multi-leaf collimator and planning system for stereotactic radiosurgery. Radiother Oncol 1999;50:325-336.

21. Xia P, Geis $\mathrm{P}, \mathrm{Xing} \mathrm{L}$, et al. Physical characteristics of a miniature multileaf collimator. Med Pliys 1999;26(1):65-70.

22. Shaw E, Kline R, Gillin M, ct al. Radiation Therapy Oncology Group: Radiosurgery quality assurance guidelines. Int J Radiat Oncol Biol Phys 1993;27(5):1231-1239.

23. Cardinale RM, Benedict $\mathrm{SH}, \mathrm{Wu} Q$, et al. A comparison of three stereotactic radiotherapy techniques; arcs vs. non-coplanar fixed fields vs. intensity modulation. Int J Radiat Ontol Biol Phys 1998;42(2):431-436.

24. Kubo HD, Pappas CT, Wilder RB. A comparison of arc-based and static mini-multileaf collimator-based radiosurgery treatment plans. Radiother Oncol 1997;45(1):89-93. 
25. Bourland JD, McCollough KP. Static field conformal stereotactic radiosurgery: Physical techniques. Int J Radiat Oncol Biol Pliys 1993;28:471-479.

26. Marks LB, Sherouse GW, Ewton JR, et al. Conformal radiation therapy with fixed shaped coplanar or noncoplanar radiation beam bouquets: A possible alternative to radiosurgery. Int J Radiat Oncol Biol Pliys 1995;33:1209-1219.

27. Laing RW, Bentley R, Nahum AE, ct al. Stereotactic radiotherapy of irregular targets: A comparison between static conformal beams and non-coplanar arcs. Radiother Oncol 1993;28(3):241-246.

28. Shiu AS, Kooy HM, Ewton JR, et al. Comparison of miniature multileaf collimation (MMLC) with circular collimation for stereotactic treatment. Int J Radiat Oncol Biol Phys 1997;37(3):679-688.

29. Hamilton RJ, Kuchnir FT, Sweeney P, et al. Comparison of static conformal field with multiple non-coplanar arc techniques for stereotactic radiosurgery or stereotactic radiotherapy. Int J Radiat Oncol Biol Pliys 1995;33(5):1221-1228.

30. Graham JD, Nahum AE, Brada M. A comparison of techniques for stereotactic radiotherapy by linear accelerator based on 3-dimensional dose distributions. Radiother Oncol 1991; 22(1):29-35.

31. Kramer BA, Wazer DE, Engler MJ, ef al. Dosimetric comparison of stereotactic radiosurgery to intensity modulated radiotherapy. Radiat Oncol Invest 1998;6:18-25.

32. Kulik C, Caudrelier JM, Vermandel M, et al. Conformal radiotherapy optimization with micromultileaf collimators: Comparison with radiosurgery techniques. Int J Radiat Oncol Biol Phys 2002;53(4):1038-1050.

33. Woo SY, Grant WH, Bellezza D, et al. A comparison of intensity modulated conformal therapy with a conventional external beam stereotactic radiosurgery system for the treatment of single and multiple intracranial lesions. Int J Radiat Oncol Biol Phys 1996;35(3):593-597.

34. Meeks SL, Buatti M, Bova FJ, et al. Potential clinical efficacy of intensity-modulated conformal therapy. Int J Radiat Oncol Biol Phys 1998;40:483-495.

35. Mohan R, Ling C. When becometh less more? Int J Radiat Oncol Biol Phys 1995;33:235-237.

36. Soderstrom S, Brahme A. Which is the most suitable number of photon beam portals in coplanar radiation therapy? Int J Radiat Oncol Biol Phys 1995;33:151-159.

37. Stein J, Mohan R, Wang XH, ct al. Number and orientations of beans in intensity-modulated radiation treatments. Med Phys 1997;24:149-160.

38. Keller-Reichenbecher MA, Bortfeld T, Levegrun S, et al. Intensity modulation with "Step and shoot" techniques using a commercial MLC: A planning study. Int J Radiat Oncol Biol Pliys 1999;45:1315-1324.

39. Ma L, Xia P, Verhey LJ, et al. A dosimetric comparison of fan beam intensity modulated radiotherapy with gamma knife stereotactic radiosurgery for treating intermediate intracranial lesions. Int J Radiat Oncol Biol Phys 1999;45:1325-1330.

40. Pirzkall A, Debus J, Haering $P$, et al. Intensity modulated radiotherapy (IMRT) for recurrent, residual, or untreated skull-base meningiomas: Preliminary clinical experience. Int J Radiat Oncol Biol Phys 2003;55(2):362-372. 
CHAPTER 4

A comparison of dose distributions of proton and photon beams in stereotactic conformal radiotherapy of brain lesions

Brigitta G. Baumert, Antony J. Lomax, Vesselin Miltchev and J. Bernard Davis

International Journal of Radiation Oncology, Biology, Physics 2001;49(5):1439-1449 
Abstract

Purpose: Micromultileaf collimators (mMLC) have recently been introduced to conform photon beans in stereotactic irradiation of brain lesions. Proton beams and stereotactic conformal radiotherapy (SCRT) can be used to tailor the dose to nonspherical targets, as most tumors of the brain are irregularly shaped. Comparative planning of brain lesions using either proton or stereotactically guided photon beams was done to assess the institution's clinically available modality for three-dimensional conformal radiotherapy.

Methods and Materials: For the photon treatment, multiple stereotactically guided uniform intensity beams from a linear accelerator were used; each conformed to a projection of the planning target volume (PTV) by a mMLC. Proton beams were delivered from an isocentrically mounted gantry, using the spot-scanning technique and energy modulation. Seven patients were scanned in a stereotactic frame; target volumes and organs at risk (OAR) were delineated with the help of MR images. Four different lesions were selected: (1) concave, (2) ellipsoid isolated, (3) superficial and close to an organ at risk, and (4) irregular complex. Dose distributions in the PTV and critical structures were calculated using three-dimensional treatment-planning systems, followed by both a quantitative (by dose-volume histogram and conformity index) and qualitative (visual inspection) assessment of the plans.

Results: A high degree of conformation was achieved with a mMLC and stereotactic uniform intensity beams with comparable conformity indices to protons for 5 out of 7 plans, especially for superficial or spherical lesions. In the cases studied, the conformity index was better for protons than for photons for complex or concave lesions, or when the PTV was in the neighborhood of critical structures.

Conclusion: The results for the cases studied, show that for simple geometries or for superficial lesions, there is no advantage in using protons. However, for complex PTV shapes, or when the PTV is in the vicinity of critical structures, protons seem to be potentially better than the fixed-field photon technique.

Keytuords: Stereotactic conformal radiotherapy, Spot-scanning technique for protons, Micronultileaf collimator, Brain tumors, Three-dimensional conformal radiotherapy. 


\section{INTRODUCTION}

The aim of three-dimensional conformal radiotherapy (3D-CRT) is to decrease the volume of irradiated normal tissue for the same target volume. This can be achieved by the use of noncoplanar beams shaped by blocks or multileaf collimators defined through a beam's-eye view or, more recently, by intensity-modulated beams.

Isodoses can be conformed to a projection of the planning target volume (PTV) and a steep dose gradient between target and normal tissue can be produced by using certain irradiation techniques, namely, linear accelerator (linac)-based stereotactic radiosurgery (SRS) or stereotactic conformal radiotherapy (SCRT), using sets of multiple intersecting noncoplanar arcs with circular collimators, which are ideally suited to spherical or ellipsoidal targets. More recently, the use of noncoplanar, fixed-intensity fields shaped by micromultileaf collimators (mMLC) has been introduced. This results in better dose conformation, particularly for irregularly shaped lesions. For these patients, the approach with arc rotation techniques is limited, as, for better conformation, multiple overlapping isocenters have to be used. This often results in a significant dose inhomogeneity. Another convergent beam technique, used for the treatment of tumors with a diameter of less than $3 \mathrm{~cm}$, is the Gamma Knife, which uses multiple 60 Co radiation sources. As with arc therapy using linacs, it is necessary to use multiple isocenters for conformation to irregular volumes resulting in a greater dose inhomogeneity within the target. However, there is some debate in the literature as to the risk of late complications as a result of dose inhomogeneity within the target volume $e^{1-5}$. Additionally, the technique is not suitable for fractionated SCRT.

The localization of dose in the Bragg peak provides an ideal characteristic for dose conformation to target volumes, and for reducing the integral dose to the patient $t^{6,7}$. Consequently, proton beams have been used in radiotherapy and radiosurgery for a number of years. The ability of protons to conform dose has been demonstrated in various comparative treatment planning studies ${ }^{8-10}$. In the spot-scanning technique, the dose to a target volume is con-formed by depositing many, individually weighted, proton Bragg peaks, distributed in three dimensions throughout the target volume.

The present study was done to assess the best modality of 3D-CRT available today in our institutions for the irradiation of brain lesions. For a series of 7 patients referred for treatment to University Hospital Zurich (UHZ), comparative planning was done using stereotactically guided uniform intensity photon beams of nominal energy ( 6 $\mathrm{MV}$ ) and protons. Various comparisons between different stereotactic radiotherapy techniques have been done, such as intercomparisons of different radiosurgery tech- 
niques with a linac ${ }^{11-14}$, of Gamma Knife and linear accelerator ${ }^{15}$, of stereotactic treatment techniques and intensity-modulated radiation therapy (IMRT) or protons ${ }^{15-19}$, but none with the use of a mMLC for photon beams. In this work, multiple static beams from a linac have been used, each conformed to the PTV by a mMLC. Similarly, the proton beams have been planned for an isocentric gantry and using the spot-scanning technique. Photon plans have been calculated using forward planning of uniform beams shaped using a mMLC and proton plans have been calculated at the Paul-Scherrer Institute (PSI) using the spot-scanning technique. The possibilities of inverse planning for either treatment modality have not been included in this comparison.

This comparison has been done on the basis of location and shape of the lesions, not of tumor type or histology. Plan comparisons were done quantitatively, by the use of dose-volume histograms (DVH) and conformity index (CI), and qualitatively, by visual inspection of the dose distribution.

\section{METHODS AND MATERIALS}

Seven patients with different tumors were selected from patients to be treated with SCRT at the UHZ. They were grouped according to tumor shape and proximity of organs at risk (OAR). The diagnoses were one optic glioma, one squamous-cell carcinoma of the paranasal sinus, one recurrent chordoma of the skull base (between brainstem and thalamus), two glioblastoma multiforme (one in close proximity to the optic radiation), one meningeoma of the sinus cavernosus, and one anaplastic astrocytoma neighboring the motor strip. The median PTV volume was $17.6 \mathrm{~cm}^{3}$; range, $3.7-32.5 \mathrm{~cm}^{3}$. Table 1 shows the different target shapes in the midplane transverse slices, as well as tumor volumes, the matching case number, and the number of radiation fields. Table 2 gives a description of tumor localizations and OAR, and summarizes prescribed dose to the PTV and maximum dose to the OAR. The given threshold dose limits for OAR in Table 2 are published data ${ }^{20,21}$. For Case 7 the particularly low-dose limit has been defined due to previous radiotherapy treatment to the area.

Patients were immobilized and scanned in a releasable stereotactic frame (BrainLAB AG, Heimstetten, Germany). Two of seven patients had an individually customized bite-block, 5 of 7 patients had a standardized fixation of the upper jaw provided by BrainLAB. The relocation accuracy of the BrainLAB mask system has been measured to be within 1-2 $\mathrm{mm}$. This is in agreement with published data and is comparable with other noninvasive fixation systems ${ }^{22,} 23$. CT images with i.v. contrast were acquired by a Somatom Plus 4 CT scanner (Siemens Medical Systems, Erlangen, 
Table 1. Tumor shapes and volumes

Lesion group

Concave

Ellipsoid

a) Superficial and

b) close to an OAR

Irregular complex

Optic radiation
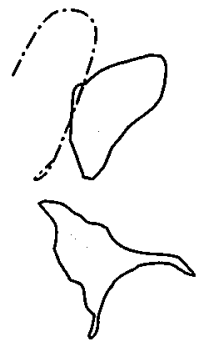

3.7

9.7

a) b) 17.6

b) 9.7

b) 32.5

24.3

11.8
No. of fields

Photon Proton Case no.

6

3

7

$\begin{array}{lll}4 & 2 & 2 \\ 4 & 1 & 6\end{array}$

SOAR: organs at risk

Table 2. Case details

Case

no. Localization

\section{Organs at risk}

Contralateral eye

Contralat. optic nerve

Chiasm (squamous cell ca.)

2

Orbit

(opric gliomn)

1) Right macula

2) Contralat. optic nerve
$D_{\max }$ OAR

Minimum possible

1) $=70 \%$ ( $\leq 36 \mathrm{~Gy})$

2) Min. possible

Minimum possible (malipuan glioma)

4 Skull base (chordomta)

5 Sinus cavernosus (meningioma)

Optic radiation Left optic nerve and chiasm

Facial nerve

Carotid artery Cochlea Brain stem

Minimum possible

\section{1) Optic nerve} ipsilat.

2) Chiasm 3) Brain stem

1) $<50 \mathrm{~Gy}$

2) $<50 \mathrm{~Gy}$

3) Min. possible

Minimum possible

$<10 \%$

7 Temporo-parietal Optic nerves and (malignamt glioma) chiasm

Abbreviations: $\mathrm{D}_{\max }$ : maximum dose; $\mathrm{OAR}$ : organs at risk 
Germany) from the base of the fiducial system (usually $2-3 \mathrm{~cm}$ below the base of the skull) to $1 \mathrm{~cm}$ above the top of the skull. The slice thickness ranged between 2 and 4 $\mathrm{mm}$, and the image matrix size was $512 \times 512$ pixels.

After transferring the CT data, target volumes, i.e., the region of contrast enhancement on $\mathrm{CT}$ and/or MRI, and OAR were delineated in all relevant slices, using the Brain-LAB treatment-planning system (TPS) with BrainSCAN v. 3.53 software. $\mathrm{MR}$ images were fused onto $\mathrm{CT}$ images both by automatic image fusion and manual alignment of the eyes and ventricles on the two image sets. This TPS uses a $3 \mathrm{D}$ algorithm with dose calculation based on tissue maximum ratios (TMR), equivalent path length (EPL), off-axis ratios, and scatter functions. Three-dimensional dose distributions have been calculated using multiple noncoplanar static beams, each conformed to a projection of the target volume by a mMLC.

The mMLC used is manually operated and based on a DKFZ-design (Deutsches Krebs Forschungs Zentrum Heidelberg, ${ }^{24}$ ). It has 2 sets of 40 nondivergent tungsten leaves with a leaf width of $1.5 \mathrm{~mm}$ at the isocenter. The $80-20 \%$ penumbra width is 2 $\mathrm{mm}$ at the isocenter. The distance between the bottom of the MMLC, and the X-ray source is $67.5 \mathrm{~cm}$. It has been shown that the use of different mMLC results in dose distributions comparable to those achieved with circular stereotactic collimators $^{25-28}$.

After photon dose calculations had been performed using the BrainLAB system, a conversion to the CADPLAN-TPS (Varian Medical Systems, Palo Alto, CA, USA) format with a matrix size of $256 \times 256$ was performed, as the matrix of BrainLAB (512 $x 512$ ) could not be read by the TPS at the PSI. Target volumes and organs at risk were then copied manually into the CADPLAN planning system. The zoom facility was used for greater accuracy. The volumes of the PTV and OAR were calculated and compared with the volumes in the BrainLAB TPS. Agreement was within $5 \%$. CT data and outlined target volumes and OAR were electronically transferred by DAT tape to the PSI for proton dose calculation. This method introduced some small differences in volumes that did not affect the conclusions of the study. To somewhat adjust for the inevitable small errors resulting from the transfer and calculation of volumes on the different planning systems, all PSI volumes have been normalized to the BrainLAB volumes.

For proton dose calculations, a 3D dose-calculation algorithm, developed at the PSI, was used. This TPS is based on the superposition of 3D pencil beam kernels, oriented in the beam coordinate system and precalculated in water-equivalent space. With the spot-scanning technique, proton pencil beams can be scanned within the target volume in three dimensions using a magnetic sweeping of the beam, a mechanical table 
motion and by interposing sequential polycarbonate sheet absorbers (range shifters) into the beam to change the position of the Bragg peak in depth. To obtain a homogeneous dose across the target volume from each incident field direction, the individual weights of the Bragg peaks are calculated using a dose based optimisation scheme ${ }^{9-31}$. Typically, each pencil beam has a lateral FWHM of about $8 \mathrm{~mm}$ in air and about $14 \mathrm{~mm}$ after $10 \mathrm{~cm}$ of tissue, due to multiple Coulomb scattering. Bragg peaks are deposited on a regular grid with a spacing of $5 \mathrm{~mm}$ along each axis. It is important to note that the optimization step in this process is used only to provide a near-homogeneous dose across the target volume for an individual field, and is simply the spot-scanning equivalent of applying a fixed scattered beam and spread-out-Bragg-peak (SOBP) across the target volume ${ }^{32,33}$. As such, this planning process cammot be considered as an inverse planning process com-parable to that applied in IMRT.

Both systems use an isocentric gantry and couch set-up. Isocenter accuracy of gantry and couch assembly is $\pm 1 \mathrm{~mm}$ for both institutes (PSI: 31 , UHZ own data: Davis JB Ph.D., unpublished data, 06/96).

Planning criteria, constraints, and dose normalization were very similar for both centers: the clinical target volume (CTV) was generally equal to the GTV and PTV. Dose distributions were nomalized at the isocenter, and $95 \%$ of the target volume was covered by the $95 \%$ isodose. For the protons, all plans were normalized to the mean PTV dose and all plans were designed such that at least $95 \%$ of the PTV was covered by the $95 \%$ isodose contour. Dose to the target and OAR were defined as shown in Table 2. At the time of calculation dose distributions were not known at the other institute.

Plan assessment was done both quantitatively and qualitatively. Qualitatively, by visual comparison of the plans; quantitatively by the use of DVH and CI. For each of the plans, DVHs were obtained for the target, the nontarget nomal brain, and organs at risk. DVH calculations were slightly different for the two TPS: BrainLAB calculates the DVH using dose values calculated in a $2-\mathrm{mm}$ grid comprising all defined structures; the TPS at PSI calculates dose values in each CT pixel in a $256 \times 256$ grid, multiplied by slice thickness.

The second criteria for quantitative plan evaluation was the conformity index, i.e., measurement of PTV coverage by $95 \%$-isodose volume (CI 95\%), defined in this case as:

$$
C I_{95}=\frac{V o l .95 \% \text { isodose }}{\text { Vol. of PTV }}
$$


This index measures the degree of conformity and is ideally $=1$, if the PTV is exactly covered by the $95 \%$ isodose volume ${ }^{34}$. Additional criteria for comparison are the volumes enclosed by isodoses of $90 \%, 80 \%, 50 \%$, and $30 \%$; the skin dose; number of applied fields; and the time for completing a treatment.

\section{RESULTS}

\section{Qualitative comparison}

Figures 1-4 show examples of isodose distributions for both treatment plans for the superficial, concave, isolated, ellipsoid, complex, and close to OAR tumor shapes. Figure 1 shows two transverse slices through the dose distributions of the SCRT and proton treatment plans for the concave tumor shape (Case 7). The level of conformation is clearly better in the proton plan, with the $95 \%$ isodose conforming along the tumor surface for protons, while for photons, it is more spherical in shape. In addition, the volume of the $30 \%$ isodose (orange volumes in the BrainLAB plan) is considerably larger for photons than for protons (pink volumes in the PSI plan).

Figure 2 shows axial slices for proton and photon plans for Case 2 (ellipsoid, isolated target) showing a similarly good target coverage and sparing of the macula by both plans. Figure 3 demonstrates a similar conformation for both techniques for an irregular and complex target close to an OAR (Case 5). Here it can be seen that the volumes of the $80 \%$ and $50 \%$ isodoses are smaller for photons. The PTV is well covered, and the brainstem is also well spared for both protons and photons. This is achieved with only 2 proton beams, whereas 5 photon beams are necessary to achieve comparable conformity. However, the $30 \%$-isodose volume for proton beams is larger than usual, due to the poor lateral dose fall-off. One reason for this could be the wider lateral penumbra, because the PSI presently does not use a collimated beam; but the main reason is the use of only 2 proton beams.

An example for an organ at risk close to the PTV is seen on Fig. 4 (Case 3): In this case, the distance between the OAR (optic radiation) and the tumor is only $2-3 \mathrm{~mm}$ and conformation to the target and sparing of the OAR are equally good for both techniques. Here the $30 \%$ isodose volume is clearly larger for the photon plan.

\section{Quantitative comparison}

Conformity indices range from 1.05 to 1.38 for protons (mean, 1.20 ), and from 1.15 to 2.03 for the photon plans (mean, 1.50). Neither modality achieved an ideal conformation. Comparing the conformity indices it can be seen that only in 2 of 7 cases 


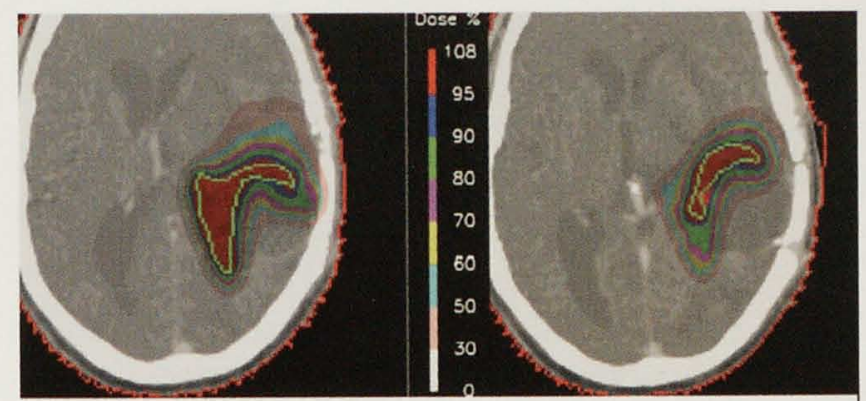

Protons

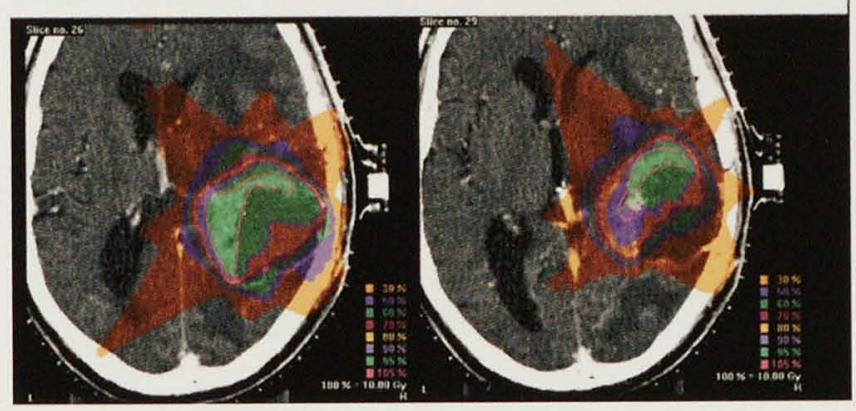

SCRT Protons

Fig. 1. Example of a concave tumor shape. The $30 \%$ isodose (orange for photons, pink for protons) is larger for the stereotactic conformal radiotherapy (SCRT) plan. Note the close conformation to the planning target volume (PTV) for the proton 95\% isodose (red volume), but not for the photon 95\% isodose (green volume).

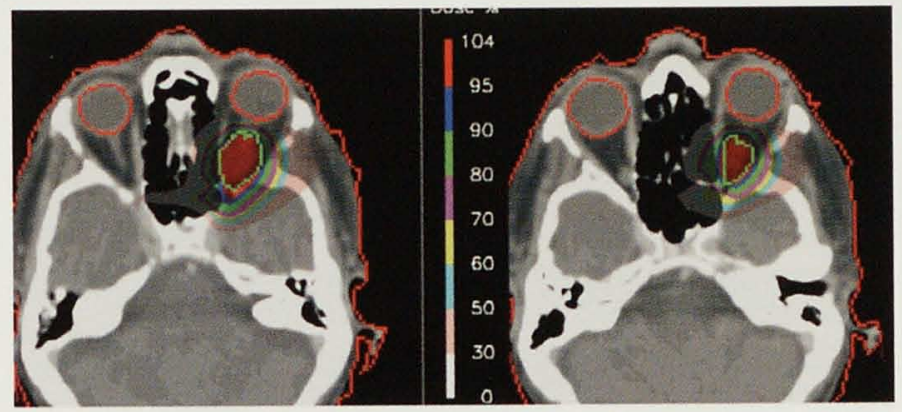

\section{Protons}

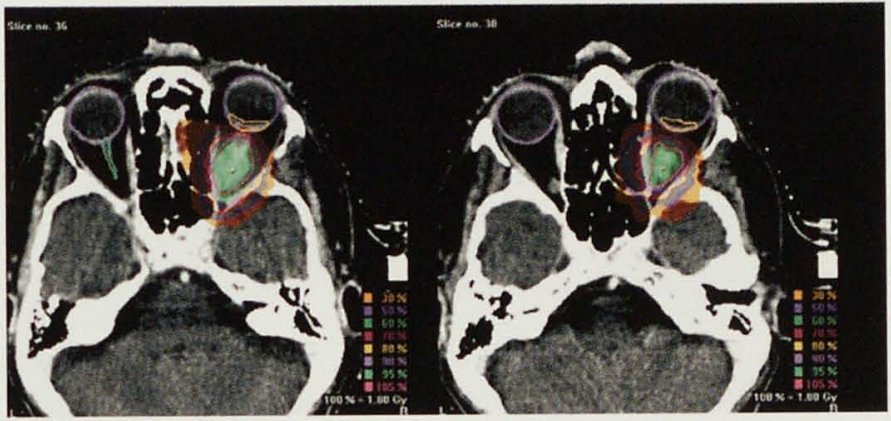

SCRT Protons

Fig. 2. Example of an ellipsoid lesion. The coverage of the planning target volume (PTV) and sparing of the organs at risk (macula) is comparable; good conformation for both plans. 


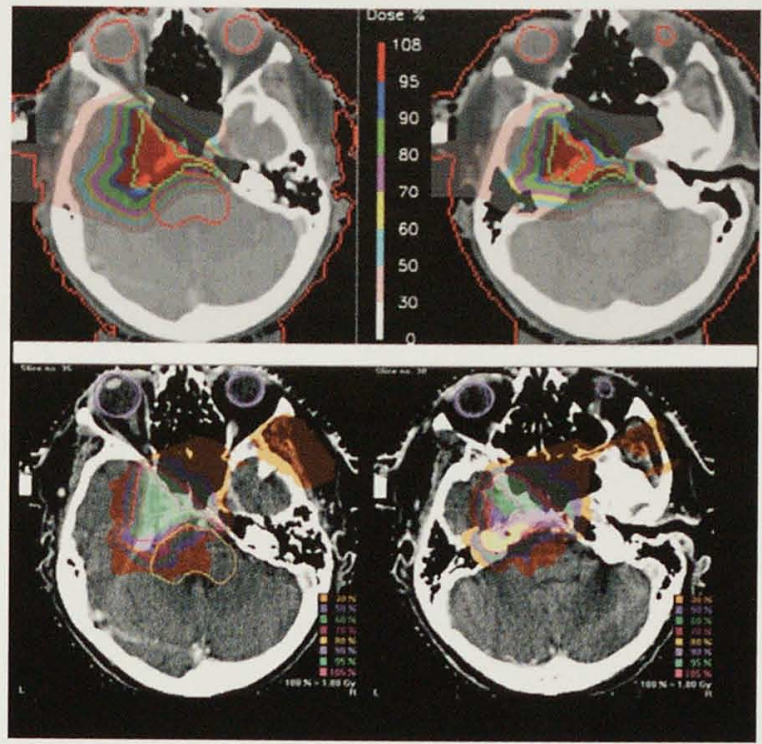

Protons

SCRT Protons

Fig. 3. Example of an irregular tumor shape; planning target volume (PTV) coverage is better for the proton plan. The proton plan uses 2 beams, the stereotactic conformal radiotherapy (SCRT) plan 5 beams. In this case, the $30 \%$ isodose volume is larger for the proton beams (pink volume) due to the poor lateral dose fall-off.

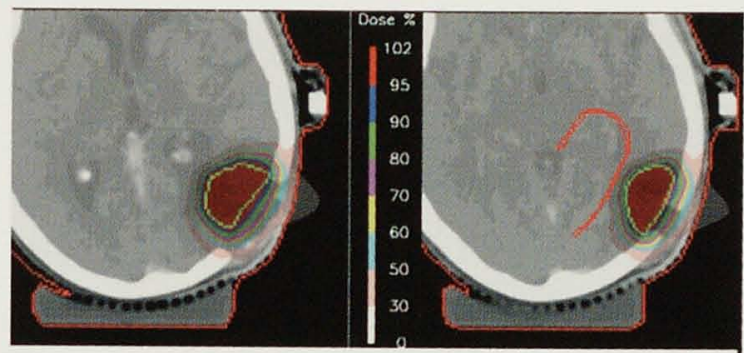

\section{Protons}

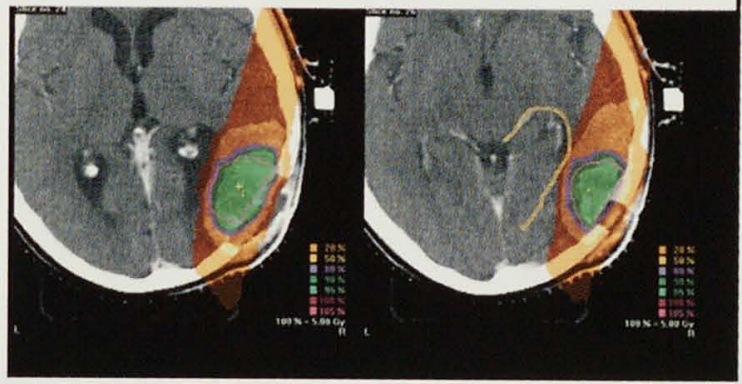

SCRT Protons

Fig. 4. Example of a neighboring organ at risk (optic radiation); conformation of isodoses to the planning target volume (PTV) and sparing of the organ at risk seem very similar. The $30 \%$ isodose volume is larger for photons. A bolus is added for protons, as the tumor is located near the surface. 
the CI $95 \%$ is clearly better for protons (Table 3). In Case 7 the photon CI $95 \%$ is 2.03 , which means that the treated volume is twice that of the PTV. Cases 3 and 6 reached equal conformation, and, in Cases 2,4 , and 5, there was only a marginal advantage to using protons. Cases 1 and 7 , which are concave and irregular targets, were the worse in comparison to the proton plan. In these cases, the photon $90 \%$ isodose volume is 1.6- to 1.7-times larger than the matching proton volume (Table 3 ), indicating that fixed-field conformal photons are unsuitable for these types of lesions. Differences are even larger for the $30 \%$ isodose. Table 3 also shows that in general proton planning uses fewer beams than stereotactically guided plans for a similar or better result. In general, the more complex or the larger a target is, the more beams are required for stereotactic planning for good coverage (see Table 1). Only for the largest target volume were more than $2-3$ proton beams necessary (Case 4).

Case 3 is a superficial lesion, and a clear disadvantage for protons can be demonstrated: for the same conformation; the proton plan uses not only more beams than expected ( 3 beams, 4 for the stereotactic approach), but a bolus is also required, although this cannot be regarded as a real disadvantage, as it is commonly used in radiotherapy.

\section{Organs at risk}

In comparing dose-volume histograms for neighboring organs at risk (Table 4) protons show better sparing especially for organs at risk (OAR) close to the target volume. For example, the macula (Case 2) and all neighboring OAR of the base of the skull, except the carotid artery in Case 4, receive less dose in comparison to the SCRT plan. This is consistent for volumes of $80 \%$ and $30 \%$ of the PTV dose. However, in Case 5 , the volumes of $80 \%$ and $30 \%$ for the right optic nerve, chiasm, and brainstem are worse for protons. This may be due to the chosen beam directions and the specific nature of the PSI beam where presently no beam collimation is used, and thus a poorer lateral fall-off is obtained than with collimated beams. In one case (Case 7), the OAR received no dose from either technique, but this may be due to the relative positions of the OAR with respect to the PTV rather than the techniques.

\section{Duration of treatment}

The daily treatment time including set-up time for both techniques is about $20 \mathrm{~min}$. 


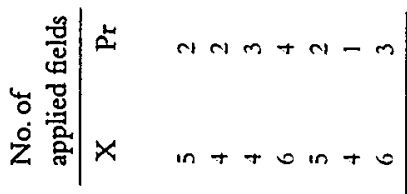

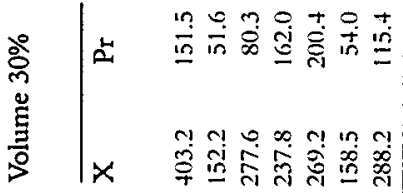

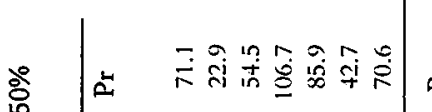

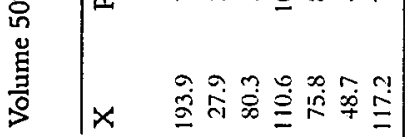

|

苛

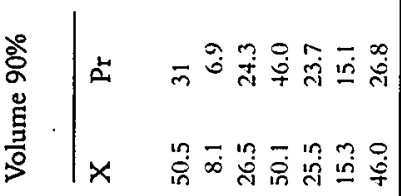

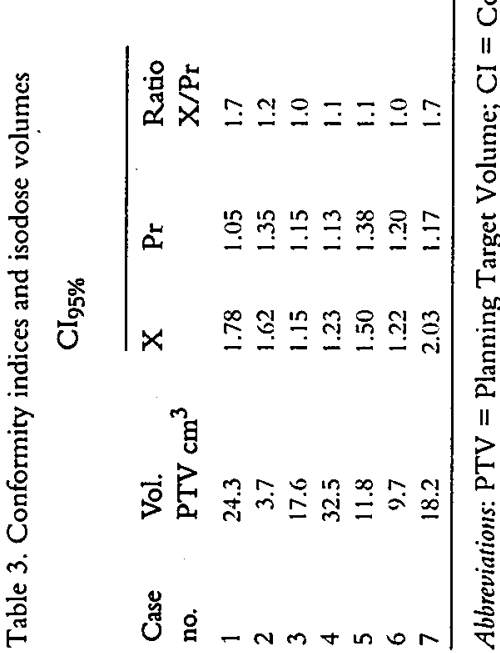

承|

00

莺|

总密|

$\Xi \Xi ', '$ '

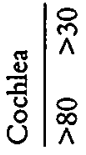

$\Xi \Xi 1,1$,

$\simeq \Perp 1,1$,

苞|

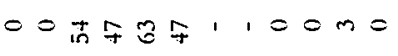

o onomi, 10000

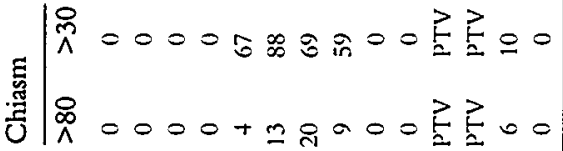

$\frac{2}{0}$

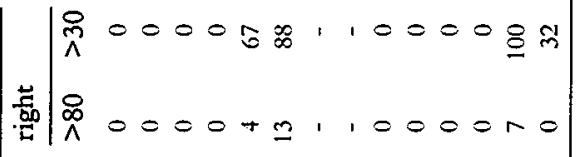

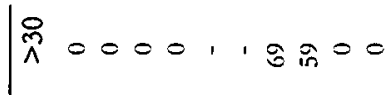

出

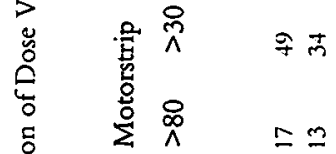

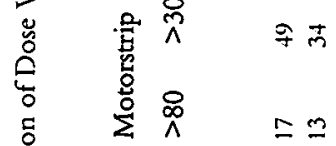

E

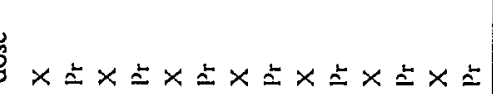

$\stackrel{\circ}{\stackrel{0}{\circ}}$ 


\section{DISCUSSION AND CONCLUSIONS}

Comparing protons and fixed-field stereotactic treatment technique with photons, on the basis of this work, it would seem that::

1. The conformity index for protons is in 2 of 7 cases clearly better than for fixed-field stereotactic photon (ratio $\mathrm{CI}_{\mathrm{ph}} / \mathrm{CI}_{\mathrm{pr}}=1.7$ ) and in the other cases slightly better or equally good, the ratio $\mathrm{CI}_{\mathrm{ph}} / \mathrm{CI}_{\mathrm{pr}}$ being in the range of 1.0 -1.2 (Table 3).

2. A high degree of conformation is achievable with mMLC and stereotactic uniform intensity beams except for the very irregular and concave lesions (Cases 1 and 7).

3. For nonconcave tumors, the volume irradiated to the $80 \%$ level of the PTV prescription dose is more favorable to photons. For instance, in 3 cases (Cases 2,5 , and 6 , Table 3 ) the $80 \%$ volume is smaller for photons and in one case it is almost the same (Case 4). The volumes receiving $50 \%$ of the PTV dose are generally reduced for protons, although the difference is small for noncomplex volumes.

4. For organs at risk in the proximity of the PTV better sparing is achieved with protons.

5. For superficial lesions a higher skin dose is given with protons. Bolus is sometimes necessary.

6. Good conformity is possible even with 1 or 2 proton beams. Stereotactic techniques need more beams for comparable conformity, but this is not necessarily a disadvantage as only a small time penalty is involved.

The results of our study are consistent with published data.

Two systems, which are established and clinically available in our institutions, spot-scanned protons and SCRT using mMLC, are compared in this study. Therefore, this comparison has been performed under the conditions of clinical practicability, such that not only an optimal target coverage and OAR avoidance were taken into consideration but also treatment time and feasibility resulting in a practical number of treatment fields.

Another parameter to be considered for this comparative study are the slightly different systems used, i.e., the accuracy of the method may not be identical for all planning steps. The redrawing of the target volumes in the CAD-PLAN TPS is a major source of inaccuracy. However, the redrawn volumes were calculated and compared with the BrainLAB volumes. Agreement was within $5 \%$. Second, slightly different dose normalization in both centers was used: dose was normalized to the isocenter for the SCRT plan, whereas, for the proton plans, the mean dose to the PTV was used. The 
calculation methods of the dose-volume histograms (DVHs) were also different, but the number of points used for its calculations were similar. However, such inaccuracies are not considered to affect the outcome and conclusions of this comparison except for very small volumes, because the differences are small and each volume was normalized to the volume of the PTV as calculated in each TPS.

In the literature, a comparison of different TPS and accuracy of methods are rarely discussed: Cardinale et al. ${ }^{16}$ state in their comparison (SRT vs. IMRT) that with the use of two different planning systems contours had to be entered separately and DVH generation algorithms were different from each other, but no estimation of accuracy deviation for the comparison is given. Another comparison between radiosurgery treatment plans of protons and X-ray beams using different TPS does not mention possible inaccuracies ${ }^{19}$, whereas Verhey et al. ${ }^{15}$, comparing Gamma Knife, photon and proton beams for SRS, concluded that copying the PTV by hand to different TPS introduced small differences in volumes. This was not considered to affect the conclusions of the comparison.

The main aspect in this study was the dose coverage of the PTV, the dose to the OAR and the dose to the normal brain tissue. The dose distribution within the target, usually measured by the homogeneity inde ${ }^{34}$ was not considered by this comparison, in that single isocenter plans have been shown to provide good dose uniformity for both proton and photon beams ${ }^{19,15}$.

The CI 95\% defined as the ratio between the volume enclosed by the $95 \%$ isodose volume and the target volume ${ }^{34}$, has been used for evaluation of the relative conformation of dose distributions resulting from both treatment modalities. The $\mathrm{CI}$ values favor protons for targets that are concave or very irregular in shape. A multiple-isocenter photon plan may have allowed a more conformal treatment, but this has not been considered in this study.

For all other targets, comparable $\mathrm{CI}$ have been achieved. For the superficial lesion (Case 3), the $\mathrm{CI}$ are equal. The proton plan was designed using three multidirectional beams to reduce the skin dose somewhat, which can be a problem in proton irradia$\operatorname{tion}^{35}$. For the other ellipsoid tumor shape (Case 6), the CI were equal, within the accuracy of the comparison. In general, the median CI values of 1.50 for the SCRT plans were comparable to the median $\mathrm{Cl}$ of 1.43 reported by Verhey et al..$^{15}$ using the Leksell Gamma Unit, and better than a median CI of 2.70 for circular collimators or 1.92 for dynamic field shaping ${ }^{36}$.

Our results are in agreement with other published results, where differences between proton and photons, for either arcs or noncoplanar shaped fixed beams, were negligi- 
ble for small volumes ${ }^{19}$. Verhey et al ${ }^{15}$ report similar and comparable results for small regular target volumes. In the linac plans, where only arc rotation was used and no mMLC treatments, photons were found to be better than the proton plans with regard to dose to the normal brain, the reason being thought to be due to the additional treatment planning margins required to account for the uncertainty of the proton stopping point. In this study, however, we have assumed the same margins for both planning modalities. A comparison of fixed-beam and multiple noncoplanar arc rotations for SRT by linear accelerator based on 3D dose distributions for spherical targets showed that the greatest sparing of normal tissue could be achieved for small volumes by a multiple-arc technique ${ }^{12}$.

For complex and irregular targets, protons clearly show a better conformation. Similar differences for the $\mathrm{Cl}$ are reported by Verhey et al. ${ }^{15}$ for irregular targets comparing proton and single isocenter linac plans, but, in their study, the authors use only arc rotation and no fixed beams and no conformal blocking or mMLC. Results were better when comparing nonstandardized complex arc plans, but at the price of an unacceptable time penalty. Significantly better conformation for irregular targets can be achieved by the use of 3D shaped noncoplanar fixed beams than by multiple- arc technique ${ }^{13,28}$. A lower dose is delivered to normal brain tissue with mini MLC-based plans than with arc-based planning ${ }^{26}$, however, some care must be taken in comparing data, because not each study uses the same comparison criteria.

For OAR close to the target, protons are generally better at sparing dose to the OAR, for both the greater than $80 \%$ and greater than $30 \%$ volume of PTV dose (Table 4). This was demonstrated in 5 of 7 cases. In one case, protons showed a higher dose to surrounding OAR, possibly due to the use of only two radiation fields. A higher number of fields may result in a better conformity. Another possible reason could be the penumbra characteristics: With the use of collimators the penumbra width will be similar to photons ${ }^{6}$.

For doses of $80 \%$ of the prescription dose, there is some advantage to the fixed-field conformal beams. At $50 \%$ of the PTV dose, there is generally an improvement for protons, although this is only marginal for the ellipsoid volumes (Cases 2 and 6), and in Case 5 there is an advantage for photons (Table 3). There is a large difference for all isodose volumes from $30 \%$ to $90 \%$ for the irregular shaped targets (Cases 1 and 7 ), always favoring the proton beam. This is in agreement with a study by Serago et al. ${ }^{19}$. The main reason for this is due to the characteristics of protons, in which no dose is delivered beyond the Bragg peak ${ }^{6}$, inevitably reducing the dose delivered to normal tissues. A recent study has shown that in a comparison of protons and IMRT X-rays, the normal tissue dose loading could be reduced on average by a factor of 2 , but that for some superficial cases, it could be reduced by as much as 4 times ${ }^{37}$. The number of 
fields used is also a factor. Although the conformity increases with a higher number of shaped fields ${ }^{11}$, so does the treated volume ${ }^{38}$ and protons are able to achieve comparable conformity with fewer fields (Table 3 ). The clinical significance of treating a larger volume to $30 \%$ of the PTV dose is not known, however. Nevertheless, the risk of cancer induction must be considered.

The implication and clinical importance of ideally con-formed dose distributions are debatable, if the prescribed doses for the OAR are below the clinical tolerance dose for late complications. Considering this aspect both methods may have the same clinical and therapeutic result.

Complication probabilities can theoretically be calculated with the use of dose-response models. A comparison based on complication and control probabilities of radiosurgery treatment modalities revealed the lowest complication probability for the larger target for protons and was less advantageous for the smaller centrally located volumes ${ }^{14}$. Nonstandard, complex linac plans (arcs) and Gamma Knife plans showed the lowest complication probabilities for most calculated cases.

In this study, we have not looked at the potential of intensity-modulated photon therapies or multiple isocenter stereotactic techniques. It would be fair to say, however, that if either of these methods were applied to the more complex target volumes in this study, the degree of conformation and normal tissue sparing would be expected to improve considerably (although at the cost of target dose homogeneity in the case of multi-isocenter treatments).

How these plans would then compare with the use of protons needs to be the subject of a separate study, but two important points should be born in mind. First, although not used here, inverse planning methods can also be applied to proton therapies ${ }^{39}$, which would also likely result in some improvement in the quality of the proton plans, particularly in the complex cases. However, the improvement in the photon dose plans is expected to be greater. Second, of the six main observations identified from this work, we would expect that the use of IMRT/multiple isocenters would affect at least three of these: Points 1 (relative conformity indices), 2 (conformation to irregular and concave target volumes), and 4 (sparing of neighboring critical structures). Although further work is clearly necessary to confirm these impressions, from this work, we can nevertheless conclude that the use of fixed, but individually shaped, photon fields provide comparable results to those obtained through the use of fixed-field proton plans for convex or simple target volumes, but that protons can conform the dose better in the case of complex shapes. For both cases, the mid-to-low doses to normal tissues are reduced through the use of protons. 
These questions will be further investigated in a new study comparing intensity-modulated proton and photon beams in radiation therapy of brain lesions.

\section{REFERENCES}

1. Nedzi LA, Kooy H, Alexander E, et al. Variables associated with the development of complications from radiosurgery of intracranial tumors. Int J Radiat Oncol Biol Phys 1991;21:591-599.

2. Flickinger JC, Lunsford LD, Linskey ME, et al. Gamma knife radiosurgery for acoustic tumors: Multivariate analysis of four year results. Radiother Oncol 1993;27(2):91-98.

3. Flickinger JC, Kondziolka D, Pollock BE, $c t$ al. Complications from arteriovenous malformation radiosurgery: Multivariate analysis and risk modeling. Int J Radiat Oncol Biol Phys 1997;38(3):485- 490 .

4. Shaw E, Scott C, Souhami L, et $d$. Radiosurgery for the treatment of previously irradiated recurrent primary brain tumors and brain metastases: Initial report of Radiation Therapy Oncology Group protocol (90-05). Int J Radiat Oncol Biol Phys 1996;34(3):647- 654.

5. Shaw E, Scott C, Souhami L, et al. Single dose radiosurgical treatment of recurrent previously irradiated primary brain tumors and brain metastases: Final report of RTOG protocol 90-05. Int J Radiat Oncol Biol Phys 2000; 47(2):291-298.

6. Webb ST. Ch. 4: The physics of proton radiotherapy. In: Webb ST, editor. The physics of three-dimensional radiation therapy. Bristol: Institute of Physics Publishing; 1993. p.172-212.

7. Koehler AM, Preston AB, Preston WM. Protons in radiation therapy. Radiology 1972; 104:191-195.

8. Mazal A, Schwartz L, Lacroix F, et al. A preliminary comparative treatment planning study for radiotherapy of age-related maculopathy. Radiother Oncol 1998;47:91-98.

9. Miralbell R, Lomax A, Russo M. Potential role of proton therapy in the treatment of pediatric medulloblastoma/primitive neuro-ectodermal tumors: Spinal theca irradiation. Int J Radiat Oncol Biol Phys 1997;38(4):805- 811.

10. Suit H, Urie M. Proton beams in radiation therapy. RevJ Natl Cancer Inst 1990;84:155-164.

11. Hamilton RJ, Kuchnir FT, Sweeney P, et al. Comparison of static conformal field with multiple non-coplanar arc techniques for stereotactic radiosurgery or stereotactic radiotherapy. Int J Radiat Oncol Biol Phys 1995;33(5):1221-1228.

12. Graham JD, Nahum AE, Brada M. A comparison of techniques for stereotactic radiotherapy by linear accelerator based on 3-dimensional dose distributions. Radiother Oncol 1991; $22(1): 29-35$.

13. Laing RW, Bentley R, Nahum AE, et al. Stereotactic radiotherapy of irregular targets: $A$ comparison between static conformal beams and non-coplanar arcs. Radiother Oncol 1993;28(3):241-246.

14. Smith V, Verhey L, Serago CF. Comparison of radiosurgery treatment modalities based on complication and control probabilities. Int J Radiat Oncol Biol Phys 1998;40(2):507-513. 
15. Verhey LJ, Smith V, Serago CF. Comparison of radiosurgery treatment modalities based on physical dose distributions. Int J Radiat Oncol Biol Phys 1998;40(2):497-505.

16. Cardinale RM, Benedict $\mathrm{SH}, \mathrm{Wu} \mathrm{Q}$, et al. A comparison of three stereotactic radiotherapy techniques; arcs vs. non-coplanar fixed fields vs. intensity modulation. Int J Radiat Oncol Biol Phys 1998;42(2):431- 436.

17. Kramer BA, Wazer DE, Engler MJ, et al. Dosimetric comparison of stereotactic radiosurgery to intensity modulated radiotherapy. Radiat Oncol Invest 1998;6:18 -25.

18. Woo SY, Grant WH, Bellezza D, et al. A comparison of intensity modulated conformal therapy with a conventional external beam stereotactic radiosurgery system for the treatment of single and multiple intracranial lesions. Int J Radiat Oncol Biol Phys 1996;35(3):593-597.

19. Serago ChF, Thornton AF, Urie MM. Comparison of proton and $\mathrm{x}$-ray conformal dose distributions for radiosurgery applications. Med Phys 1995;22(12):2111-2116.

20. Emami B, Lyman J, Brown A, et al. Tolerance of normal tissue to therapeutic radiation. Int J Radiat Oitcol Biol Phys1991;21:109-122.

21. Tholen AM, Meister A, Bernasconi PP, et al. Radiotherapy for choroidal neovascularization in age-related macular degeneration. A pilot study using low-versus high-dose photon beam radiation. Ophthalmologe 1998;10:691-698.

22. Hodapp N, Nanko N, Rohner F, ct al. Quality assurance for non-invasive patient fixation during stereotactic convergent beam irradiation. Acta Neurochir Suppl (Wien) 1994;62:101104.

23. Willner J, Flentje M, Bratengeier K. CT simulation in stereotactic brain radiotherapy-analysis of isocenter reproducibility with mask fixation. Radiother Oncol 1997;45(1):83- 88.

24. Schlegel W, Pastyr O, Bortfeld $T$, et al. Stereotactically guided fractionated radiotherapy: Technical aspects. RadiotherOncol 1993;29:197-204.

25. Cosgrove VP, Jahn U, Pfaender M, et al. Commissioning of a micro multi-leaf collimator and planning system for stereotactic radiosurgery. Radiother Oncol 1999;50:325-336.

26. Kubo HD, Pappas CT, Wilder RB. A comparison of arc-based and static mini-multileaf collimator-based radiosurgery treatment plans. Radiother Oncol 1997;45(1):89 -93.

27. Xia P, Geis P, Xing L, et al. Physical characteristics of a miniature multileaf collimator. Med Phys 1999;26(1):65-70.

28. Shiu AS, Kooy HM, Ewton JR, et al. Comparison of miniature multileaf collimation (MMLC) with circular collimation for stereotactic treatment. Int J Radiat Oncol Biol Phys 1997; 37(3):679-688.

29. Lomax AJ, Pedroni E, Schaffner B, et al. 3D treatment planning for conformal proton therapy by spot scanning. In: Faulkner K, Carey B, Crellin A, editors. Quantitative imaging in oncology. London: British Institute of Radiology; 1996. p. 67-71.

30. Scheib S. Spot-scanning mit Protonen: Experimentelle Resultate und Therapieplanung. Thesis, Eidgenoessische Hochschule, Zuerich.; 1994.

31. Pedroni E, Bacher R, Blattmann $\mathrm{H}$, et al. The $200-\mathrm{MeV}$ proton therapy project at the Paul Scherrer Institute. Conceptual design and practical realization. Med Phys 1995; 22(1):37-53.

32. Koehler AM, Schneider RJ, Sisterson JM. Range modulators for protons and heavy ions. Nuc Instrum Meth 1975;131:437-440. 
33. Koehler AM, Schneider RJ, Sisterson JM. Flattening of proton dose distributions for large field radiotherapy. Med Pliys 1977; 4:297-301.

34. Shaw E, Kline R, Gillin M, et al. Radiation Therapy Oncology Group: Radiosurgery quality assurance guidelines. Int J Radiat Oncol Biol Phys 1993;27(5):1231-1239.

35. Arimoto $\mathrm{T}, \mathrm{Maruhashi} \mathrm{N}$, Takada $\mathrm{Y}$, et al. Acute skin reactions observed in fractionated proton irradiation. Radiat Med 1989;7(1):23-27.

36. Nedzi LA, Kooy HM, Alexander ED, et al. Dynamic field shaping for stereotactic radiosurgery: A modeling study. Int J Radiat Oncol Biol Phys 1993;25:859 -869.

37. Lomax AJ, Bortfeld T, Goitein G, et al. A treatment planning inter-comparison of proton and intensity modulated photon radiotherapy. Radiother Oncol 1999;51:257-271.

38. Knoeoes T, Kristensen RN, Nilsson P. Volumetric and dosimetric evaluation of radiation treatment plans: Radiation conformity index. Int J Radiat Oncol Biol Phys 1998;42(5):11691176.

39. Lomax AJ. Intensity modulated methods for proton therapy. Phys Med Biol 1999; 44:185-205. 


\section{CHAPTER 5}

Dose conformation of intensity modulated stereotactic photon beams, proton beams and intensity modulated proton beams for intracranial lesions

Brigitta G. Baumert, Ian A. Norton, Antony J. Lomax and J. Bernard Davis

International Journal of Radiation Oncology, Biology, Physics 2004; 60(4):1314-1324 
Abstract

Purpose: This study evaluates photon beam intensity-modulated stereotactic radiotherapy (IMSRT) based on dynamic leaf motion of a micromultileaf collimator ( $\mathrm{MMLC}$ ), proton beams, and intensity modulated proton therapy (IMPT) with respect to target coverage and organs-at-risk.

Material and Methods: Dose plans of six stereotactically treated patients were re-calculated for IMSRT by use of the same field setup and an inverse planning algorithm. Proton and IMPT plans were calculated anew. Three different tumor shapes, multifocal, ovoid and irregular, were analyzed:, as well as dose to organs-at-risk (OAR) in the vicinity of the planning target volume (PTV). Dose distributions were calculated from bean-setup data for a manual mMLC for stereotactically guided conformal radiotherapy (SCRT), a dynamic mMLC for IMSRT, the spot scanning technique for protons, and a modified spot scanning technique for IMPT. SCRT was included for a part of the comparison. Criteria for assessment were PTV coverage, dose-volume histograms (DVH), volumes of specific isodoses and the dose to OAR. Results: Dose conformation to the PTV is equally good for all 3 techniques and tumor shapes considered. The volumes of the $90 \%$ and $80 \%$ isodose were comparable for all techniques. For the $50 \%$ isodose volume, a divergence between the 2 modes was seen. In 3 cases, this volume is smaller for IMSRT, and for the 3 other cases, it is smaller for IMPT. This difference was even more pronounced for the volumes of the $30 \%$ isodose; IMPT shows further improvement over conventional protons. OAR in concavities (e.g., the brain stem) were similarly well spared by protons and IMSRT. IMPT spares critical organs best. Fewer proton beams are required to achieve similar results.

Conclusions: The addition of intensity modulation improves the conformality of mMLC-based SCRT. Conformation of dose to the PTV is comparable for IMSRT, protons, and IMPT. Concerning the sparing of OAR, IMSRT is equivalent to IMPT, and IMPT is superior to conventional protons. The advantage of protons lies in the lower integral dose.

Keywords: Stereotactic conformal radiotherapy, protons, micro-multileaf collimator, brain tumors, skull base, three-dimensional conformal radiotherapy, intensity modulation. 


\section{INTRODUCTION}

Major computer hard-and software developments during the past decade have made full three-dimensional photon radiotherapy planning possible. Tools like beam's-eye-views, dose-volume histograms, three-dimensionally (3D) dose calculations and computer-driven multi-leaf collimators are the basis for three-dimensional conformal radiotherapy. The addition of beam-intensity modulation is the most recent and advanced achievement in this area. Stereotactically guided conformal radiotherapy (SCRT) adds highly accurate repositioning and irradiation, which enables the extensive use of noncoplanar beams. As was the case for 3D treatment with photons, sophisticated treatment planning and highly accurate dose delivery were also introduced for particle-beam radiotherapy. Intensity-modulated therapy (IMPT) was then the next logical development.

Both proton beams and intensity-modulated photon radiotherapy (IMRT) can fully cover the tumor volume in three dimensions, which results in the possibility of increasing the dose to the target volume. Improved three-dimensional conformal radiotherapy (3D-CRT) results in a reduction of serious late complications. A reduction of $41 \%$ for patients treated before 1986 to $20 \%$ for patients treated between 1987 and 1992 has been achieved by use of particle beams ${ }^{1}$. Better avoidance of organs-at-risk (OAR) was also shown for IMRT-based planning ${ }^{2}$. Recent reports on the treatment of skull-base meningioma with SCR $\mathrm{T}$ in which both photons and protons were used, as well as IMRT, seem to indicate that the treatment is effective and safe ${ }^{3-5}$. To increase local tumor control, a dose escalation is necessarily accompanied by the prerequisite of additional sparing of non-target tissue. For IMRT, this prerequisite is achieved through defined dose goals and constraints for the tumor and normal tissue. For proton beams, this is achieved through the physical fact that the range of protons in tissue is finite and terminates in the Bragg peak, which results in a reduced integral dose to normal tissue $e^{6-7}$.

Classical clinical indications for proton therapy are tumors in close proximity to critical structures, especially those partially enclosed by the tumor itself, and tumors that need a high dose of radiation for local control, such as skull-base chordomas ${ }^{8}$. An increase in total dose of about $10 \%$ to $15 \%$ by use of a combination of proton and photon treatment may also result in increased local control for meningiomas ${ }^{9}$. With intensity modulation now clinically available for routine treatment, the same indications apply for radiotherapy with photons ${ }^{5}$. This development translates into the use of intensity-modulated stereotactic radiotherapy (IMSRT) for base of skull lesions, mostly for irregularly shaped targets close to critical structures, such as meningiomas of the cavernous sinus. The brainstem and the pituitary gland often lie in a concavity partially enclosed by the meningioma. IMRT has the ability to conform the dose to 
concavities ${ }^{2,10}$. With computer-controlled micro multileaf collimator (mMLC) systems, IMRT can now be extended to small intra-cranial volumes ${ }^{11}$.

In a previous publication, 3D-CRT dose distributions of photon and proton beams for stereotactic irradiations of brain lesions have been compared ${ }^{12}$. This comparison showed a clear advantage for protons in 2 out of 7 cases studied. This finding applies to 2 very irregular and concave cases. In a further 3 cases, (round and non-concave tumor shapes) no definite advantage was seen for protons, and in 2 cases, photons were superior. As for round and non-concave lesions, both techniques reached a high degree of conformation. This study extends the previous comparison to both photon and proton intensity-modulated beams to examine possible improvements for mainly irregular and concave shapes. Results of differences between stereotactic radiotherapy and intensity-modulated stereotactic radiotherapy are discussed separately ${ }^{13}$.

Because the magnitude of a physical benefit of either method is not yet clear, this study looks at the best dose distributions obtainable from IMSRT and IMPT. We investigate how treatment of irregular and concave-shaped tumors and those close to OAR (e.g., base of skull tumors) can be optimized. The expected benefit of IMRT for both radiation forms is studied, and advantages of each modality are analyzed with respect to a better avoidance of surrounding normal brain and, therefore, lesser risk of long term radiation side effects. A subgroup of tumors for which IMRT might be advantageous was chosen from a series of patients referred for stereotactic radiotherapy. Standard treatment plans that made use of stereotactically-guided conformal radiotherapy (SCRT) and both IMPT and IMRT were compared. As the current study is a modeling study, comparison is based on tumor location and shape only, without consideration of tumor histology or fractionation (fractionated or single dose treatment). The idea of this study was to assess the possible benefit of adding intensity modulation to 3D stereotactic and proton planning. Thus, variables such as number of beam angles for photons were kept to a minimum. This study is a 3-way inter-comparison of the effects of intensity-modulated stereotaxy with photons, conventional protons, and intensity-modulated proton beams on tumors mainly located in the skull base. Few studies that compare standard photons, IMRT and protons, and IMRT and IMPT for treatment of tumors of the brain or the skull base reported in the literature ${ }^{12,14-17}$. 


\section{METHODS AND MATERIALS}

\section{Overview}

Six patients were selected on the basis of tumor location and shape from a group of 10 patients previously treated with SCRT. Patient characteristics have been published elsewhere ${ }^{13}$ but for completeness are listed in Table 1 . Four patients had a tumor located in the base of the skull ( 3 meningioma, one craniopharyngeoma), 1 patient had a multifocal recurrent falx meninigoma, and 1 a nasopharyngeal recurrence of a tonsil carcinoma. The number of fields used for each treatment technique and volumes are given in Table 1 . The corresponding target shapes and descriptions are shown in Table 2. Skull-base tumors are mainly irregular and, therefore, represent somewhat larger tumor volumes; the median gross tumor volume (GTV) is $10 \mathrm{~cm}^{3}(3$ $\left.-18 \mathrm{~cm}^{3}\right)$. The GTV was expanded three-dimensionally by a 3-mm margin, which resulted in a median planning target volume (PTV) of $29.5 \mathrm{~cm}^{3}\left(17-43 \mathrm{~cm}^{3}\right)$ (Table 1).

Table 1. Patient data

\begin{tabular}{|c|c|c|c|c|c|c|c|}
\hline \multirow[b]{2}{*}{$\begin{array}{l}\text { Case } \\
\text { no. }\end{array}$} & \multirow[b]{2}{*}{$\begin{array}{l}\text { Diagnosis and anatomic } \\
\text { site }\end{array}$} & \multirow[b]{2}{*}{$\begin{array}{l}\text { Prescribed } \\
\text { SCRT Dose }\end{array}$} & \multicolumn{3}{|c|}{ No. of fields } & \multicolumn{2}{|c|}{$\begin{array}{l}\text { Target } \\
\text { volume }\left(\mathrm{cm}^{3}\right)\end{array}$} \\
\hline & & & SCRT & IMSRT & $\begin{array}{l}\text { Protons } \\
\text { and } \\
\text { IMPT }\end{array}$ & GTV & PTV \\
\hline 1 & $\begin{array}{l}\text { Skull base meningioma, } \\
\text { parasellar }\end{array}$ & 30) $\times 1.8=54 \mathrm{~Gy}$ & 5 & 5 & 3 & 4 & 17 \\
\hline 2 & $\begin{array}{l}\text { Skull base meningioma, } \\
\text { parasellar }\end{array}$ & 30) $\times 1.8=54 \mathrm{~Gy}$ & $\begin{array}{l}7(5+2 \\
\text { boost ficlds) }\end{array}$ & 5 & 3 & 14 & 40 \\
\hline 3 & $\begin{array}{l}\text { Recurrence squamous cell } \\
\text { carcinona of the tonsil, } \\
\text { foramen magnum, pre-irradi- } \\
\text { ated }\end{array}$ & $5 \times 5=25 \mathrm{~Gy}$ & 6 & 6 & 3 & 18 & 43 \\
\hline 4 & $\begin{array}{l}\text { Skull base meningiona, } \\
\text { suprasellar }\end{array}$ & $30 \times 1.8=54 \mathrm{~Gy}$ & 5 & 5 & 3 & 8 & 28 \\
\hline 5 & Multifocal meningiona, falx & $1 \times 20$ Gy & 5 & 6 & 3 & 3 & 17 \\
\hline 6 & $\begin{array}{l}\text { Carniopharyngeoma, } \\
\text { suprasellar }\end{array}$ & $30 \times 1.8=54 \mathrm{~Gy}$ & 5 & 5 & 3 & 13 & 32 \\
\hline
\end{tabular}

Abbreviations: $\mathrm{GTV}=$ gross target volume; $\mathrm{PTV}=$ planning target volume; $\mathrm{SCRT}=$ stereotactic conformal radiotherapy IMSRT = intensity modulated stereotactic radiotherapy; IMPT = intensity modulated protons.

The SCRT technique has been described elsewhere ${ }^{12}$. Head fixation was by means of a special relocatable stereotactic frame and a standardized upper jaw fixation (1 patient), an additional customized bite-block (4 patients), or a frame for radiosurgery (1 patient) (BrainLAB AG, Heimstetten, Germany). A CT scan with i.v. contrast medium was performed by use of a slice thickness and distance of $3 \mathrm{~mm}$ for the whole 
Table 2. Details of target volume

Lesion group
Multifocal
$\begin{aligned} & \text { Mainly ovoid on axial views, concave on } \\ & \text { sagittal view }\end{aligned}$

skull, starting from the base of the fiducial system. The tumor was outlined on T1-weighted MR scan, which had been fused to a contrast-enhanced CT. GTV, PTV, and OAR were prepared for the proton treatment planning system (TPS) by the CADPLAN TPS (Varian Medical Systems, Palo Alto, CA, USA). A direct transfer of the outlined volumes to the proton TPS from the BrainLAB TPS was not possible because of incompatible data formats. After electronic transfer to the proton TPS, volumes were compared to ensure an agreement within $5 \%$. Absolute volumes from the TPS were used for comparison of normal tissue irradiation. GTV, PTV, and OAR comparisons utilized percent of total volume. Total-volume calculations from the BrainLAB TPS were considered to be more accurate because of the finer grid used.

\section{Dose calculation}

For treatment planning, $\mathrm{CT}$ and $\mathrm{MR}$ data and the BrainLAB TPS with BrainSCAN V5.0 software were used. This TPS uses an algorithm with a $1.5 \times 1.5 \mathrm{~mm}$ pencil beam for a high resolution in dose distribution. IMSRT planning uses a variant of the maximum-likelihood estimator method of statistical parameter estimation, the dynamically penalized likelihood method ${ }^{18,19}$. Beam collimation was by the BrainLAB M3 mMLC, which has 26 leaf pairs with varying leaf widths. Details and characteristics of this MLC have been described elsewhere ${ }^{11,20}$. The effective width of the $80 \%$ to $20 \%$ penumbra is less than $3.0 \mathrm{~mm}$ for all field sizes ${ }^{20}$. Optimization of IMSRT was performed by application of the smallest possible calculation grid and predefined OAR dose constraints. Dose constraints for smaller OAR were better achieved with the smallest possible grid size. Beams for the optimization were calcu- 
lated using a 2-mm or 3-mm grid. The first step was the calculation of SCRT dose plans for a manually operated mMLC (Deutsches Krebs Forschungs Zentrum Heidelberg, ${ }^{21}$ ). This $\mathrm{mMLC}$ has a $80 \%$ to $20 \%$ penumbra width of $2 \mathrm{~mm}$ at the isocenter.

For the calculation of proton dose intensity matrices, a 3D dose-calculation algorithm for spot-scanned proton therapy, developed at the Paul-Scherrer Institute, was used. In contrast to conventional, or passive scattering, proton therapy ${ }^{22,23}$, spot scanning is based on the application of three-dimensionally distributed and individually weighted Bragg peaks ${ }^{24}$. To obtain a homogeneous dose across the target volume from each incident field direction, the individual weights of the Bragg peaks are optimized by the use of a dose-based optimization scheme ${ }^{24-26}$. After a field direction is defined, a subset of all possible Bragg peaks (distributed on a 4- $\mathrm{mm} \times 4-\mathrm{mm} \times$ $4.5-\mathrm{mm}$ grid) that are within the target volume are selected for the optimization, and their weights are optimized by application of a dose-based iterative optimization scheme ${ }^{25,26}$.

This process has the sole purpose of delivering a uniform dose to the target. A multiple-field plan is then constructed through the simple linear and weighted addition of these individually homogenous, single-field dose distributions. IMPT plans are calculated in a similar way; the critical difference is that the subsets of Bragg peaks for all fields are optimized simultaneously, with the more relaxed constraint that only the total dose from all fields together should provide a homogenous dose across the target volume. In addition, for IMPT, dose-volume constraints can also be defined to critical structures in a similar way as for IMRT ${ }^{27}$.

For SCRT and IMSRT, dose distributions were normalized at the isocenter. For protons and IMPT, all plans were normalized to the mean PTV dose. Requirements were the same for both centers and all treatment planning techniques; $100 \%$ of the target volume was to be covered by the $95 \%$ isodose, but a $95 \%$ coverage in $90 \%$ of the volume was deemed acceptable if the PTV was adjacent to an OAR. The dose constraints, weighting factors, and penalties used were dependent on the planning systems and were set for each plan such that the constraints to the target could be fulfilled while sparing the defined critical organs as much as possible. The chosen dose maxima are relative and dependent on the total dose and fractionation scheme prescribed. For the optic nerve and chiasm, the dose tolerated was the minimum possible but less than $45 \mathrm{~Gy}$; for the pituitary, it was less than $40 \mathrm{~Gy}$; for the eye, less than $5 \mathrm{~Gy}$; for the brain stem, less than $56 \mathrm{~Gy}$; and for the motor cortex and trigeminal nerves, the mimimum possible. The particularly low-dose limit for the spinal cord with minimum dose possible but less than $40 \mathrm{~Gy}$ was set because of a previous curative radiotherapy to the same area (Case 3 , see also Table 1 ). 


\section{Treatment planning}

Intensity modulated stereotactic RT plans were based on the optimized SCRT plans. For SCR T planning, the beam angles and the number of fields were defined by conforming multiple noncoplanar static beams to the projections of the PTV in each beam's-eye-view (BEV). The optimized SCRT plan was then taken further in a second step for IMSR T planning. The IMSR T inverse planning process optimized critical-organ dose-sparing while maintaining PTV dose conformity. Dose-volume constraints could be defined directly within the dose-volume histograms (DVH) graphs, which also allows the definition of relative weighting between the PTV and different OAR.

For IMPT, an inverse planning technique was applied to the spot-scanning technique in 3 dimensions. Dose constraints and maximum doses were applied as for the photon plans. Further weighting factors for OAR were taken into account for the calculation. For the proton planning, the same planning steps apply as for the stereotactic photon plans. The beam geometry was identical for both proton plans; the IMPT plans were based on the forward planned proton plans.

\section{Comparison of treatments and analysis of conformity}

All plans were compared by use of DVH analysis and conformity indices (CI) for a quantitative plan assessment. A qualitative assessment was done by visual comparison of dose distributions. For the DVH analysis, specific volumes were compared: volumes enclosed by the $30 \%$ to $90 \%$ isodoses (V30\%-V90\%). DVH calculations were slightly different for the IMRT calculation algorithms. BrainLAB calculates $D V H$ by predefinable grid sizes during the optimization process. These grid sizes were set to 2 or $3 \mathrm{~mm}$ grid size for SCRT and IMSRT. The proton and IMPT TPS calculated DVH dose by use of a pixel-to-volume conversion factor. Total volume of normal tissue for both planning systems consisted of the whole-brain volume minus the PTV.

Planning target coverage was measured by the use of a conformity index (CI). For this study, a new CI first published by Paddick ${ }^{28}$, which also takes into account the location of the prescription dose volume relative to the target volume, was used. This factor is not considered by the RTOG index ${ }^{29}$. The Paddick index is described by the ratio of the target volume within the prescribed isodose volume and not simply by the ratio of the PTV and the volume of the prescribed isodose. The mathematical difference is shown in the following equations. Both indices measure the degree of conformity and are ideally $=1$.

$$
C I_{95 \%}=\frac{P I V}{T V} \quad \text { (RTOG index) }
$$




$$
C I_{95 \%}=\frac{T V_{P I I^{2}}}{T V \times P I V} \quad \text { (Paddick index) }
$$

where PIV $=95 \%$ isodose volume, $\mathrm{TV}=$ volume of $\mathrm{PTV}$.

Additionally, the Paddick index has been shown to be more sensitive to volume differences in an analysis of conformal BEV planning and intensity-modulated planning for stereotactic radiotherapy $13,30,31$. The RTOG index could not detect differences in conformality between 2 planning methods, whereas the Paddick index showed a clear difference in favor of intensity modulation.

\section{RESULTS}

\section{Quantitative comparison}

Conformality: No treatment modality reached the ideal conformation of 1.0 (mean scores). The RTOG and Paddick indices each show a good and similar discrimination between the 3 techniques. The mean scores of the Paddick index for IMSRT is 0.84 (range $0.78-0.90)$, for protons $0.86(0.80-0.91)$ and for IMPT $0.85(0.78-$ 0.89 )(Table 3). The mean values of the RTOG index are 1.03, 1.04 and 1.03 for IMSRT, protons and IMPT, respectively. The RTOG CI is for most more than 1.0, except for Case 1 for protons $(0.98$, Case 2 for IMSR T (0.95), and Case 6 for IMPT (0.99)(Table 3)). These differences are small and can be caused by only a few pixels being outside of the $95 \%$ isodose line.

Regarding the different tumor shapes, IMPT is less conformal than conventional proton beams for 2 ovoid and 2 irregular tumors (Cases 1 and 6 and Cases 2 and 4 (Table 3)). Within the group of IMPT-planned cases, Cases 3 and 6 (ovoid shapes) have the highest Paddick CI, but Case 6 shows higher CI scores for IMSRT and protons. For the irregular tumor shapes (Cases 2 and 4), IMSRT and IMPT produce a very similar conformality.

Table 4 shows the coverage of the PTV by the $95 \%$ isodose. Coverage is seen to be worse for SCRT in all cases and best for protons and IMPT for Cases 2 and 4 (irregular shapes) and the multifocal Case 5 . The ovoid shapes are best covered by IMSRT. Generally, differences are small. Dose maxima are highest for IMPT with values of $110 \%$ (Table 4).

In Table 5, the volumes of the 50\% to $90 \%$ isodoses (V50-90\%) are larger for standard proton plans for Cases 3 to 6 compared to the intensity-modulated plans. For Case 1 , the proton V $90 \%$ is smaller than the IMPT V $90 \%$, and for Case 2, the IMSRT V $50 \%$ 
Table 3. Conformity indices

\begin{tabular}{|c|c|c|c|c|c|c|c|c|}
\hline \multirow{2}{*}{$\begin{array}{l}\text { Case } \\
\text { no. }\end{array}$} & \multicolumn{2}{|l|}{ IMSRT } & \multicolumn{2}{|l|}{ Prot } & \multicolumn{2}{|l|}{ IMPT } & \multirow{2}{*}{$\begin{array}{l}\text { Ratio } \\
\text { (Paddick) } \\
\text { Prot/ } \\
\text { IMPT }\end{array}$} & \multirow{2}{*}{$\begin{array}{l}\text { Ratio } \\
\text { (Paddick) } \\
\text { IMSRT/ } \\
\text { IMPT }\end{array}$} \\
\hline & Paddick & RTOG & Paddick & RTOG & Paddick & RTOG & & \\
\hline 1 & 0.82 & 1.06 & 0.84 & 0.98 & 0.78 & 1.02 & 1.08 & 1.05 \\
\hline 2 & 0.80 & 0.95 & 0.86 & 1.08 & 0.81 & 1.05 & 1.06 & 0.99 \\
\hline 3 & 0.88 & 1.02 & 0.85 & 1.06 & 0.89 & 1.03 & 0.96 & 0.99 \\
\hline 4 & 0.83 & 1.03 & 0.88 & 1.00 & 0.87 & 1.02 & 1.01 & 0.95 \\
\hline 5 & 0.78 & 1.08 & 0.80 & 1.09 & 0.83 & 1.04 & 0.96 & 0.94 \\
\hline 6 & 0.90 & 1.05 & 0.91 & 1.00 & 0.89 & 0.99 & 1.02 & 1.01 \\
\hline Mean & 0.84 & 1.03 & 0.86 & 1.04 & 0.85 & 1.03 & 1.02 & 0.99 \\
\hline
\end{tabular}

Abbreviations: $\mathrm{CI}=$ Conformity Index of $95 \%$ isodose volume, $\mathrm{PIV}=$ prescription isodose volume; $\mathrm{TV}=$ Volume of PTV. IMSRT = intensity modulated stereotactic radiotherapy; Prot = protons; $I M P T=$ intensity modulated protons.

Notc: Paddick index: $\mathrm{CI}_{y_{5}}=\mathrm{TV}_{\mathrm{piv}} 2 / \mathrm{TV} \times$ PIV; $\mathrm{RTOG}$ index: $\mathrm{CI}_{y_{5}}=\mathrm{TV} / \mathrm{PIV}$

Table 4. Percent of prescription isodose covering the PTV and maximum dose within the PTV

\begin{tabular}{|c|c|c|c|c|c|c|c|c|}
\hline \multirow{2}{*}{$\begin{array}{l}\text { Case } \\
\text { no. }\end{array}$} & \multicolumn{4}{|c|}{$95 \%$ Isodose covering the target (\%) } & \multicolumn{4}{|c|}{ Maximum dose within target (\%) } \\
\hline & $\overline{\text { SCRT }}$ & IMSRT & Prot & IMPT & SCRT & IMSRT & Prot & IMPT \\
\hline 1 & 77.4 & 93.5 & 91.0 & 89.2 & 100 & 105 & 108 & 110 \\
\hline 2 & 78.3 & 87.2 & 96.3 & 92.2 & 104 & 108 & 106 & 108 \\
\hline 3 & 79.5 & 94.9 & 94.7 & 95.7 & 102 & 106 & 107 & 108 \\
\hline 4 & 80.7 & 92.4 & 93.9 & 94.1 & 102 & 107 & 107 & 107 \\
\hline 5 & 67.6 & 92.0 & 93.4 & 93.1 & 105 & 105 & 106 & 110 \\
\hline 6 & 79.3 & 97.1 & 95.3 & 94.3 & 101 & 103 & 106 & 107 \\
\hline
\end{tabular}

Abbreviations: SCRT $=$ stereotactic conformal radiotherapy; IMSRT $=$ intensity modulated stereotactic radiotherapy; Prot $=$ protons; $\mathrm{IMPT}=$ intensity modulated protons

Table 5. Isodose volumes

\begin{tabular}{|c|c|c|c|c|c|c|c|c|c|c|c|c|}
\hline \multirow{2}{*}{$\begin{array}{l}\text { Case } \\
\text { no. }\end{array}$} & \multicolumn{3}{|c|}{ Volume $\left(\mathrm{cm}^{3}\right) 90 \%$} & \multicolumn{3}{|c|}{ Volume $\left(\mathrm{cm}^{3}\right) 80 \%$} & \multicolumn{3}{|c|}{ Volume $\left(\mathrm{cm}^{3}\right) 50 \%$} & \multicolumn{3}{|c|}{ Volume $\left(\mathrm{cm}^{3}\right) 30 \%$} \\
\hline & IMSRT & Prot & IMPT & IMSRT & Prot & IMPT & IMSRT & Prot & IMPT & IMSRT & Prot & IMPT \\
\hline 1 & 22.2 & 21.3 & 21.5 & 24.9 & 29.6 & 27.8 & 57.4 & 57.5 & 50.0 & 189.3 & 93.1 & 78.3 \\
\hline 2 & 47.2 & 50.3 & 48.5 & 59.4 & 66.0 & 61.7 & 138.7 & 127.9 & 110.6 & 365.8 & 218.2 & 187.2 \\
\hline 3 & 50.4 & 58.2 & 54.0 & 60.7 & 79.0 & 69.8 & 125.2 & 150.4 & 120.4 & 359.6 & 246.7 & 204.0 \\
\hline 4 & 33.7 & 35.7 & 34.5 & 41.1 & 52.8 & 50.7 & 86.4 & 102.2 & 98.0 & 262.3 & 168.7 & 155.5 \\
\hline 5 & 22.2 & 25.8 & 23.5 & 28.8 & 38.8 & 33.6 & 58.3 & 79.8 & 68.5 & 133.0 & 127.7 & 99.9 \\
\hline 6 & 36.7 & 39.6 & 37.9 & 42.8 & 53.6 & 49.8 & 78.2 & 96.7 & 86.6 & 278.5 & 151.4 & 150.9 \\
\hline Mean & 35.4 & 38.5 & 36.7 & 43.0 & 53.3 & 48.9 & 90.7 & 102.4 & 89.0 & 264.8 & 167.6 & 146.0 \\
\hline
\end{tabular}

Abbreviations: $\mathrm{IMSRT}=$ intensity modulated stereotactic radiotherapy; Prot $=$ protons; $\mathrm{IMPT}=$ intensity modulated protons 
Mean Volume Normal Tissue Irradiated $\left(\mathrm{cm}^{3}\right)$

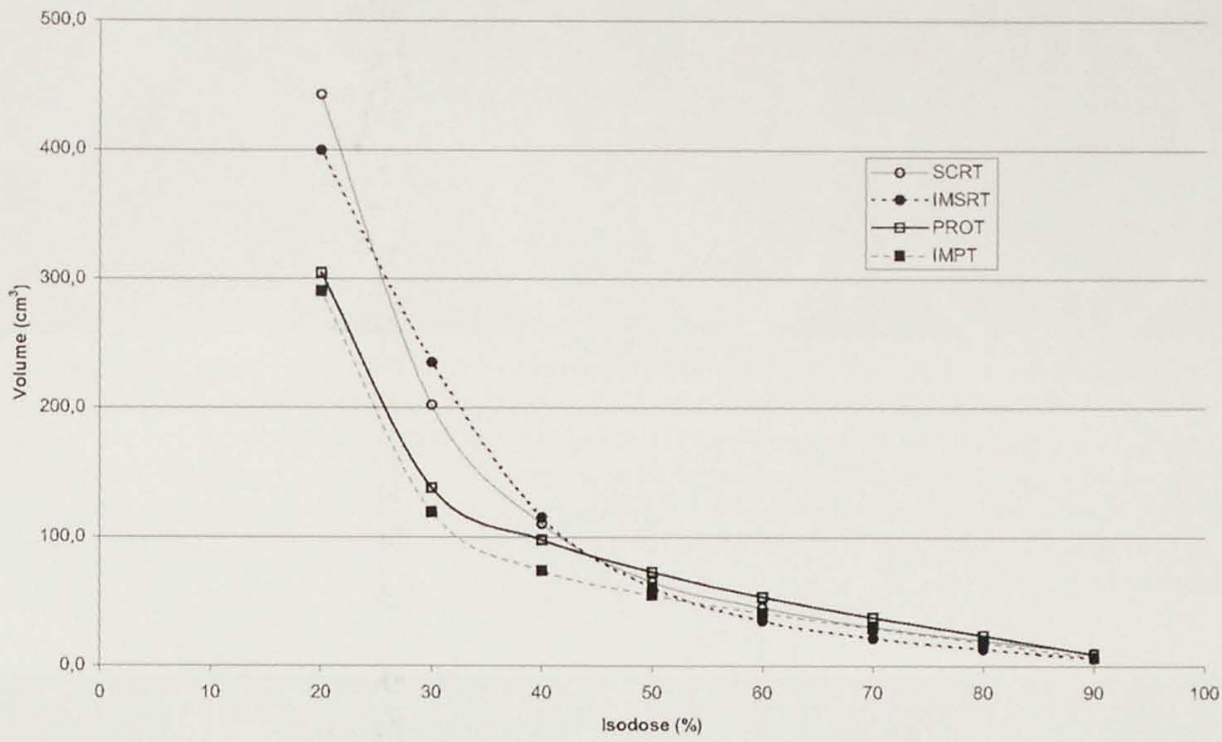

Fig 1. The average volume of normal tissue irradiated of all patients and all 3 planning methods is shown. The $y$-axis is defined as the percentage of total volume irradiated. The $x$-axis is defined as the percentage of different isodose volumes separated by $10 \%$ steps.

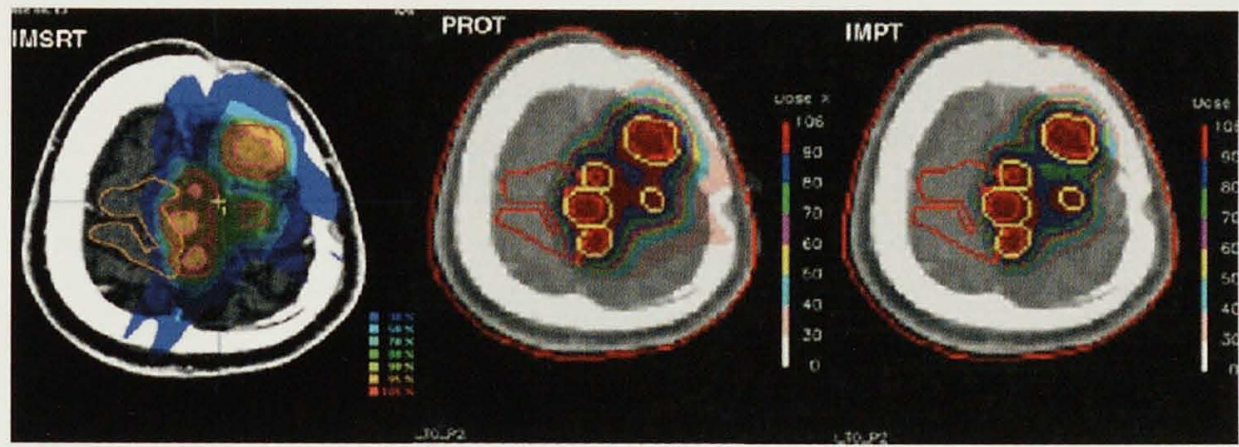

Fig 2. Example of a multifocal tumor volume (Case 5). Isodose distributions of all 3 planning methods are shown. The high dose isodoses are reduced in the intensity modulated plans (yellow for IMSRT, in red for IMPT). IMSRT $=$ intensity-modulated stereotactic radiotherapy; PRT $=$ proton-based planning with conventional protons; IMPT $=$ intensity-modulated proton-beam therapy. Isodose color wash lines are defined in the right-hand corner for each image. 


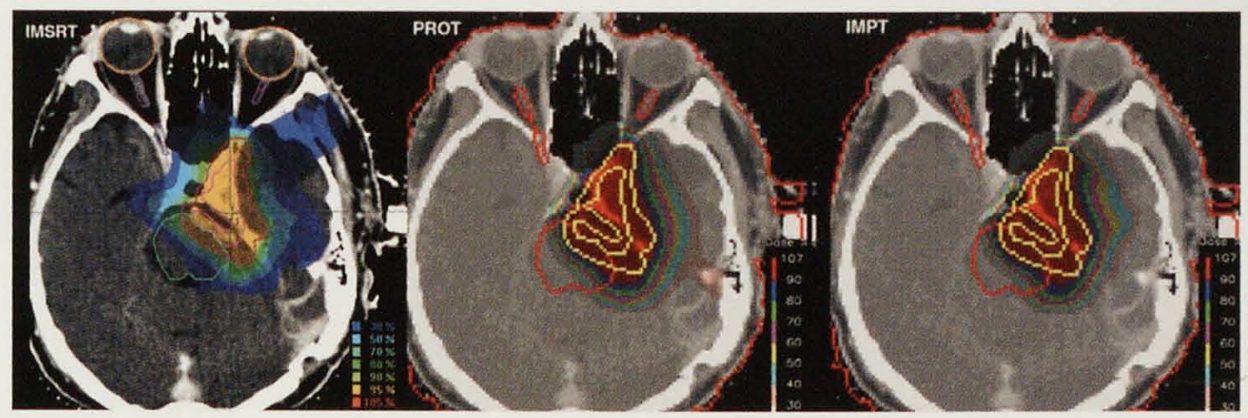

Fig 3. Example for an irregular skull base tumor (Case 4). Isodose distributions of all 3 planning methods are shown. The 50\% and 30\% isodose volumes are largest for the IMSRT plan (light and dark blue) and lowest for IMPT (yellow and pink for proton plans). The small, elongated isodose areas in form of a tail on the intensity-modulated plans seem to be caused by dose compensation in individual beams. IMSRT = intensity-modulated stereotactic radiotherapy; PRT = proton-based planning with conventional protons; IMPT $=$ intensity-modulated proton-beam therapy. Isodose color wash lines are defined in the right-hand corner for each image.

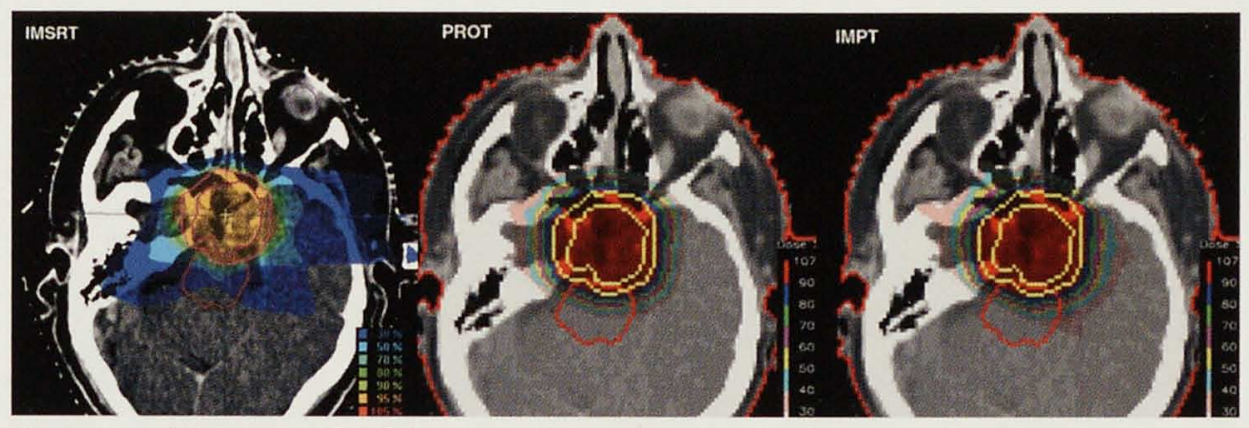

Fig 4. Example for an ovoid skull base tumor (Case 6). Isodose distributions of all 3 planning methods are shown. The $50 \%$ and $30 \%$ isodose volumes are largest for the IMSRT plan (light and dark blue) and lowest for IMPT (yellow and pink for proton plans). The $80 \%$ isodose volume is larger for the IMSRT plan (light green) compared with both proton plans (dark blue). IMSR T = intensity-modulated stereotactic radiotherapy; PRT = proton-based planning with conventional protons; IMPT $=$ intensity-modulated proton-beam therapy. Isodose color wash lines are defined in the right-hand corner for each image.

is larger than the proton and IMPT V $50 \%$. For Cases 2 to 6 , the V $90 \%$ and V80\% are smallest for IMSR T. The advantages of V50\% are split between IMSR T and IMPT. For 3 cases (4-6) the V50\% is smallest for IMSRT; and for the Cases 1, 2, and 3, it is smallest for IMPT (Table 5). As expected, the advantage of using protons is greatest for the $30 \%$ and lower isodose volumes. Adding intensity modulation to protons further reduces the $30 \%$ and lower isodose volumes (Table 5 ), thus reducing the integral dose.

Organs-at-risk $(O A R)$ and mean dose to normal tissue: Overall, when comparing DVH of $\mathrm{OAR}$ close to the tumor, dose values for all 3 planning modalities are similar for 
most of the cases considered. Generally, the addition of intensity modulation to the protons reduces the dose to OAR (Table 6). The dose to the left optic nerve in Case 4 (irregular) or to the brain stem (Case 3, ovoid) from standard proton and IMPT techniques are greater compared with IMSRT. However, to better spare the ipsilateral optic nerve (situated close to the PTV), a substantial amount of dose is redistributed to the contralateral optic nerve (Case 4, irregular) for IMSRT. For Case 2, the pituitary, an organ lying in a concave GTV, is better spared by IMPT. Intensity modulation techniques achieved a reduction of dose to the chiasm in 3 cases: for Cases 2 and 4 by IMSRT and for Case 6 by IMPT. Figure 1 shows the mean volumes of the $20 \%$ isodoses to the $90 \%$ isodoses. These volumes are an indication of the amount of normal tissue irradiated outside the PTV. The amount of normal tissue irradiated with IMPT is the lowest between the V20\% and the V50\%. The lowest value of the $80 \%$ isodose volume is observed for IMSRT and, therefore, seems to have the steeper dose fall-off at the PTV margin, whereas the lowest less than $30 \%$ volumes are always seen with IMPT.

Number offields: In this study, similar levels of conformality (Table 3) are reached for proton planning with fewer fields: protons generally need 3 fields and photons need a median of 5 fields (Table 2). ). An investigation of proton plans with more than 3 beams was not performed for this study.

\section{Qualitative comparison}

Individual isodose distributions are shown for 3 patients in Figs. 2-4. Figure 2 shows isodose distributions for the multifocal case (Case 5). Dose conformation of the $95 \%$ and $80 \%$ isodoses is clearly better in both intensity-modulated plan; the dose is reduced between the tumor foci. Figure 3 represents an irregular case (Case 4): conformation is better for both intensity-modulated plans. The extension of the $30 \%$ isodose is the largest for the IMSRT plan. The dose falloff from the PTV to the brainstem is slightly steeper for IMSRT. Figure 4 shows an ovoid tumor shape (Case 6), where the dose falloff, especially laterally, is better for protons and IMPT, thus reducing dose to normal tissue. 
岂

㺃|

)

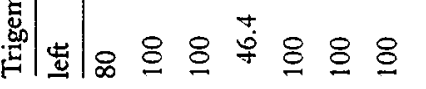

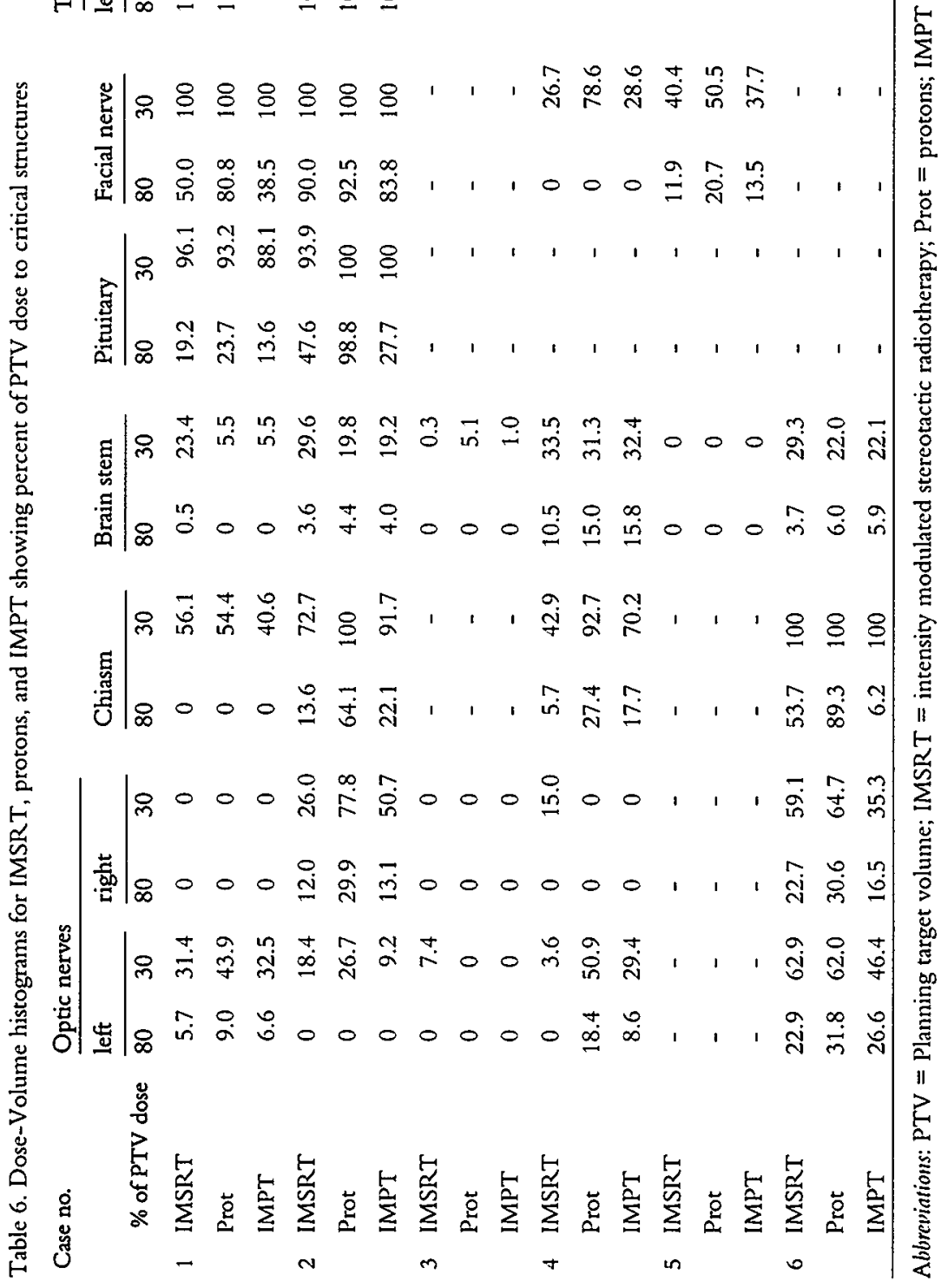




\section{DISCUSSION}

An equivalent high level of conformation occurred for all 3 modalities. The Paddick index ${ }^{28}$, which takes into account the localization of the PTV in relation to the prescription isodose volume, was more sensitive to small differences in conformality than the RTOG index. However, as differences are small and would be insignificant when the CI of the RTOG is used ${ }^{29}$, the results are considered equal. Similar results were found by others who compared intensity-modulated $X$-ray plans, protons, and IMPT while investigating nonstereotactic plans for larger paranasal tumors and prostate cancer ${ }^{14-16,32}$.

Overall, proton based planning resulted in a slightly better conformality. No pronounced differences in conformality for different tumor shapes between IMSRT, protons, and IMPT were seen. For ovoid tumor shapes, IMPT planning seems to be of no advantage. The $\mathrm{CI}$ values of 1 irregular and 2 ovoid cases were equivalent for IMSRT and IMPT (ratios of 0.99 and 1.01). This similar result of conformality for protons and photons is due to the addition of intensity modulation to both methods ${ }^{13,15}$. However, it contradicts some published data, which showed that protons were always better than stereotactic photon plans ${ }^{12,33}$. The difference in favor of protons was more pronounced for larger and irregular tumor volumes.

A steeper dose falloff was reached with IMPT in the high-dose isodose volumes. The $\mathrm{V} 90 \%$, the V80\%, and the V $50 \%$ were larger for standard proton plans. The addition of intensity modulation to SCRT and protons reduces the V80\% and the V30\%; the largest gain in this study was seen for IMSRT. The reasons for this may be the higher number of fields used for photon planning or different weighting for both planning methods. Another comparison of IMSRT vs. SCRT reached the same conclusion for medium-sized convex tumor shapes, namely, an overall improvement in conformality but at the cost of higher dose to $\mathrm{OAR}^{34}$. In contrast to our findings that the addition of intensity modulation improves the $\mathrm{CI}$ for all shapes, others ${ }^{35,36}$ found an advantage only for larger and irregular shaped tumors.

Beside conformality and target coverage, the second main endpoint of this study was the dose to OAR and normal brain tissue. In general, all OAR adjacent to the tumor were equally well spared by all 3 treatment modalities. The use of intensity modulation for protons further reduced dose to OAR. The improved sparing of OAR, however, was reached at the cost of a dose redistribution to other OAR (e.g., the contra-lateral optic nerve). This tradeoff was seen in intensity-modulated planning for both SCRT and protons, especially for irregular tumor shapes. The reason for the latter could be most presumably be found in the higher-weighted constraint for 
tumor coverage. Both the reduced low-to-medium dose to OAR and the lower V30\% have been reported previously for cranial and extracranial targets ${ }^{16,32,37}$.

Intensity-modulated radiotherapy was suggested as a suitable technique to better spare OAR lying within concavities of the PTV. As shown in this study, IMRT better spares OAR and may, thus, reduce the risk of long-term radiation side effects (e.g., the dysfunction of the pituitary). Even if only a part of the pituitary can be spared, a clinical gain may be accomplished. Recent data describe a partial volume effect for the pituitary with an endocrine dysfunction being unlikely if the dose is less than 50 cobalt equivalent Gray (CGE) to the anterior part of the gland ${ }^{38}$. A posterior pituitary dysfunction with doses between $50 \mathrm{CGE}$ and $70 \mathrm{CGE}$ was not observed. The prescribed dose limits to the normal tissue were reached in this study, and the total dose to part of the pituitary was kept well beyond $50 \mathrm{~Gy}$, especially for the anterior part of the gland. This was also caused by the growth pattern of skull-base meningiomas, which grow dorsally around the sella turcica. As a conclusion, we can say that the use of intensity modulation can spare even small OAR lying within concavities of a skull base lesion. The same is true for the brain stem, but numbers are less clear here as usually only a small part of the brain stem borders the PTV.

The price for the higher conformality achieved with IMSRT is a redistribution of dose between the OAR in some cases and a slight increase in the integral dose in other cases. An increase of integral dose, that is, a larger volume of normal brain tissue irradiated with a low dose, would increase the risk of secondary malignancies $39,40,41,42$. As a consequence, the dose to normal tissue should always be kept to a minimum. Radiation-induced cancer incidence is assumed to be a function of dose for all solid tumors. In a review of 53 patients with reconstructed dosimetry, a significantly higher number of malignancies was found within the volume that received less than 6 $\mathrm{Gy}^{39}$. The volumes smaller than 6 Gy would correspond to the $10 \%$ isodose volume $(5.4 \mathrm{~Gy})$ in this study. The low-dose isodose volumes of $30 \%$ to $10 \%$ are generally increased with intensity-modulated photons. Doses lower than $6 \mathrm{~Gy}$ have been reported to induce meningioma in normal tissue that receives doses of about $1 \mathrm{~Gy}$ after irradiation of tinea capitus ${ }^{40}$. One might argue that those cases reported were treated with nonconformal techniques and orthovoltage irradiation, but more recent reports can also be found. Six cases of carcinogenesis have been reported 6 to 7 years after radiosurgery ${ }^{43-47}$. Three glioblastomas occurred within the high-dose region, but one was described originating within the scatter or penumbra region ${ }^{43}$. Thus, the expected gain from use of IMPT instead of protons or IMSR T lies in normal tissue sparing.

The amount of normal tissue irradiated with a low mean dose ( $20 \%$ isodose) was reduced and not increased with IMSRT compared with SCRT, as one normally 
would expect, whereas the V30\% was increased (see Figure 1). This finding implies that the use of intensity modulation for stereotactically guided photons would reduce the integral dose in the low-dose area. This expectation is not supported in the literature, where the use of intensity modulation for stereotactic treatments usually reported to result in a better conformation of the target at a cost of a larger penumbra and a higher dose in the $10 \%$ to $50 \%$ volumes $^{48-50}$. One reason for this observation could be the fact that comparative planning studies did not examine very low dose levels less than $30 \%$ for nontarget tissue. Another reason is the fact that for this study, the same number of beam angles for both SCRT and IMSRT planning was used to examine the influence of intensity modulation on conformality. The improvement in terms of reduced morbidity (e.g., pituitary dysfunction) vastly outweighs the increased risk of carcinogenesis, especially where the clinical impact of low doses to larger normal tissue is unknown.

With respect to differences found between treatment planning methods, both SCRT and IMSRT plans were done by an individual highly experienced in the field of stereotactic radiotherapy planning. IMSR $T$ planning was based on the field setup defined by BEV sparing of OAR. For inverse planning, the optimization algorithm used can compensate to a certain extent no ideal beam directions. The best plans, however, result from the combination of well-selected beam orientations based on $\mathrm{BEV}$ avoidance of OAR and intensity modulation based on appropriately chosen dose constraints.

Both treatment delivery systems compared in this study, spot-scanned protons and stereotactically guided radiotherapy, are established in clinical practice. Intensity modulation of protons and SCRT was planned but not used in treatment. Planning was performed under the assumption of a theoretical clinical application and clinical practicability. Furthermore, differences between both systems were taken into account: small differences in volumes caused by redrawing of targets, different dose normalization, and calculation methods (e.g., grid sizes and DVH calculations). Because overall differences are small, they are not considered to affect the conclusions of this study. The problem of comparing different systems is similarly discussed detailed in our previous study 12 and in the literature $e^{2,31}$.

This study was performed to determine whether a benefit is achieved by use of intensity modulation for tumors with an irregular shape and concavities, which often enclose OAR such as the brain stem or the pituitary. The same field setup as used in daily practice was considered. Kulik et al. ${ }^{49}$ determined the number of fields by application of a stochastic method as the optimization algorithm: 5 fields optimized by BEV showed only small differences compared with the same setup for IMSRT in 
terms of CI. This finding supports other published reports, which have shown that 4 to 6 hand-selected fields preserve the same quality of treatment ${ }^{2,51-53}$.

Concerning the number of proton beams, it has been shown that IMRT of photons planned with 9 fields compared with forward-planned protons with a median of 3 beams ( 2 to 4 beams) resulted in essentially the same conformation of the $95 \%$ to $70 \%$ dose level and a considerably reduction of lower dose levels ${ }^{14}$. In another comparison of intensity-modulated photons and protons for a patient with a nasopharyngeal carcinoma, the same number of photon and proton beams ( 9 coplanar beams) were chosen. However, the use of 9 proton fields was assumed to be suboptimal for total integral dose $\mathrm{e}^{15}$. Thus, a higher number of proton fields would eventually increase the total integral dose, as it does for photon planning.

From this work, we can conclude that the addition of intensity modulation improves planning modalities of both SCRT and conventional proton beams independently of tumor shape, as well as the sparing of OAR, especially those situated within target concavities. The latter is partially at the cost of higher dose in other OAR, which is the nature of IMRT planning, where dose reduction to some areas of the treated volume results in a possible dose increase in another area. Normal tissue complication probability (NTCP) calculations for radiosurgery and IMSRT were comparable for both treatment modalities because current models of tissue tolerance consider the larger low-dose volumes to be clinically insignificant ${ }^{35}$. However, these models do not necessarily provide accurate values for complications, because adequate data with long follow-up are not yet available. The use of NTCP-based planning and radiobiologic modeling may provide further knowledge to help choose the best modality when these data will be available.

Concerning integral dose, IMPT showed the best sparing of normal brain tisue. The question of clinical impact of higher conformality needs to be addressed in further studies, but sophisticated planning has a place in treating benign and pediatric tumors or preirradiated recurrences, where both conformity and reduction of the integral dose are warranted in order to reduce late side effects.

\section{REFERENCES}

1. Castro JR, Linstadt DE, Bahary JP, $c t$ al. Experience in charged particle irradiation of tumors of the skull base: 1977-1992. Int J Radiat Oncol Biol Phys 1994;29(4):647-55.

2. Verhey LJ. Comparison of Three-Dimensional Conformal radiation therapy and intensity-modulated radiation therapy systems. Seminars Radiat Oncol 1999, 9:78-98. 
3. Jalali R, Loughrey C, Baumert B, et al. High precision focussed irradiation in the form of fractionated stereotactic conformal radiotherapy (SCRT) for benign meningiomas predominantly in the skull base location. Clin Oncol ( $R$ Coll Radiol). 2002;14(2):103-9.

4. Vernimmen FJ, Harris JK, Wilson J, ct al. Stereotactic proton beam therapy of skull base meningiomas. Int J Radiat Oncol Biol Phys 2001;49(1):99-105.

5. Pirzkall A, Debus J, Haering P, ct al. Intensity modulated radiotherapy (IMRT) for recurrent, residual, or untreated skull-base meningiomas: preliminary clinical experience. Int $J$ Radiat Oncol Biol Phys. 2003;55(2):362-372.

6. Webb St. The physics of proton radiotherapy. In: Webb St, editor. The physics of three-dimensional radiation therapy. Bristol and Philadelphia: Institute of Physics Publishing; 1993. p. 172-212.

7. Koehler $\mathrm{AM}$, Preston $\mathrm{AB}$, Preston WM. Protons in radiation therapy. Radiology 1972;104:191-195.

8. Miralbell R, Lingood RM, Monte $S$, ct al. The role of radiotherapy in the treatment of subtotally resected benign meningiomas. J Neuro-Oncol 1992;13:157-164.

9. Noel G, Habrand JL, Mammar H, et al. Highly conformal therapy using proton component in the management of meningiomas. Preliminary experience of the Centre de Protontherapie d'Orsay. Strallenther Onkol 2002;178(9):480-485.

10. Benedict $\mathrm{SH}$, Cardinale $\mathrm{RM}, \mathrm{Wu} \mathrm{Q}$, et al. Intensity-modulated stereotactic radiosurgery using dynamic micro-multileaf collimation. Int J Radiat Oncol Biol Phlys 2001;50(1): 751-758.

11. Xia $\mathrm{P}, \mathrm{Geis} \mathrm{P}, \mathrm{Xing} \mathrm{L}$, et al. Physical characteristics of a miniature multileaf collimator. Med Phys. 1999;26(1):65-70.

12. Baumert BG, Lomax AJ, Miltchev V, Davis JB. A comparison of dose distributions of proton and photon beams in stereotactic conformal radiotherapy of brain lesions. InI J Radiat Biol Phys 2001;49(5):1439-1449.

13. Baumert BG, Norton IA, Davis JB. Intensity modulated stereotactic radiotherapy versus stereotactic conformal radiotherapy for the treatment of meningioma predominantly located in the skull base. Int J Radiat Biol Phys 2003;57(2):580-92.

14. Lomax AJ, Bortfeld Th, Goitein G, et al. A treatment planning inter-comparison of proton and intensity modulated photon radiotherapy. Radiother Oncol 1999;51:257-271.

15. Lomax AJ, Goitein $\mathrm{M}$, Adams J. Intensity modulation in radiotherapy: photon versus protons in the paranasal sinus. Radiother Oncol 2003;66(1):11-18.

16. Miralbell R, Cella L, Weber D, Lomax AJ. Optimizing radiotherapy of orbital and paraorbital tumors: intensity modulated $X$-ray beams versus intensity modulated proton beams. Int J Radiat Oncol Biol Plys 2000;47:1111-1119.

17. Bolsi A, Fogliata A, Cozzi L. Radiotherapy of small intracranial tumours with different advanced techniques using photon and proton beams: a treatment planning study. Radiother Oncol 2003;68(1):1-14.

18. Llacer J. Inverse radiation treatment planning using the Dynamically Penalized Likelihood method. Med Phys 1997;24(11):1751-1764. 
19. Llacer J, Solberg TD, Promberger C. Comparative behavior of the dynamically penalized algorithm in inverse radiation therapy planning. Phys Med Biol 2001;46(10): 2637-63.

20. Cosgrove VP, Jahn U, Pfaender M, t al. Commissioning of a micro multi-leaf collimator and planning system for stereotactic radiosurgery. Radiother Oncol 1999;50:325-336.

21. Schlegel W, Pastyr O, Bortfeld Th, ct al. Stereotactically guided fractionated radiotherapy: technical aspects. Radiother Oncol 1993;29:197-204.

22. Koehler AM, Schneider RJ, Sisterson, JM. Range modulators for protons and heavy ions. Nuc Instrum. Mcth. 1975;131: 437-440

23. Koehler AM, Schneider RJ, Sisterson J.M. Flattening of proton dose distributions for large field radiotherapy. Med. Phys. 1977; 4: 297-301

24. Pedroni E, Bacher R, Blattmann $\mathrm{H}$, ct al. The $200-\mathrm{MeV}$ proton therapy project at the Paul Scherrer Institute: conceptual design and practical realization. Mcd Phys 1995;22(1):37-53.

25. Lomax AJ, Pedroni E, Schaffner B, et al. 3D treatment planning for conformal proton therapy by spot scanning. In: K Faulkner, B Carey, A Crellin, RM, editors. Quantitative Imaging in Oncology. Harrison. London: British Institute of Radiology;1996. p. 67-71.

26. Scheib S. Spot-scanning mit Protonen: Experimentelle Resultate und Therapieplanung. Thesis, Eidgenoessische Hochschule, Zurich; 1994.

27. Lomax A. Intensity modulation methods for proton radiotherapy. Phys Med Biol 1999;44:185-205.

28. Paddick I. A simple scoring ratio to index the conformity of radiosurgical treatment plans. $J$ Neurosurg 2000; (Suppl 3) 93:219-222.

29. Shaw E, Kline R, Gillin M, ct al. Radiation Therapy Oncology Group: Radiosurgery quality assurance guidelines. Int J Radiat Oncol Biol Phys 1993;27(5):1231-9.

30. Nakamura JL., Verhey LJ, Smith V, et al. Dose conformity of gamma knife radiosurgery and risk factors for complication. Int J Radiat Oncol Biol Pliys 2001;.51(5):1313-1319.

31. Nakamura JL, Pirzkall A, Carol MP, et al. Comparison of intensity-modulated radiosurgery with gamma knife radiosurgery for challenging skull base lesions. Int J Radiat Oncol Biol Phys 2003;55(1):99-109.

32. Cella L, Lomax A, Miralbell R. New techniques in hadrontherapy: intensity modulated proton beams. Phys Med 2001;17 (Suppl 1):100-2.

33. Serago ChF, Thornton AF, Urie MM. Comparison of proton and x-ray conformal dose distributions for radiosurgery applications. Med Phys 1995;22(12):2111-2116.

34. Khoo VS, Oldham M, Adams EJ, ct al. Comparison of intensity-modulated tomotherapy with stereotactically guided conformal radiotherapy for brain tumours. Int J Radiat Oncol Biol Plys 1999;45(2):415-425.

35. Meeks SL, Buatti M, Bova FJ, ot al. Potential clinical efficacy of intensity-modulated conformal therapy. Int J Radiat Oncol Biol Phys 1998;40:483-495.

36. Cardinale RM, Benedict $\mathrm{SH}, \mathrm{Wu} Q$, ct al. A comparison of three stereotactic radiotherapy techniques; arcs vs. noncoplanar fixed fields vs. intensity modulation. Int J Radiat Oncol Biol Phys 1998;42(2):431-436.

37. Leavitt DD, Watson $G$, Tobler $M$, et al. Intensity-modulated radiosurgery/radiotherapy using a micromultileaf collimator. Int J Radiat Oncol Biol Phys 2001, 26:143-150. 
38. Pai HH, Thornton A, Katznelson L, et al. Hypothalamic/pituitary function following high-dose conformal radiotherapy to the base of skull: demonstration of a dose-effect relationship using dose-volume histogram analysis. Int J Radiat Oncol Biol Phys 2001; 49(4):1079-92.

39. Doerr W, Hermann Th. Second primary tumors after radiotherapy for malignancies. Strallenther Onkol 2001;178:357-62.

40. Ron E, Mosan B, Boice JD, et al. Tumors of the brain and nervous system after radiotherapy in children. N Engl J Mcd 1988;319:1033-1039.

41. Neglia J, Meadows A, Robinson L, et al. Second malignant neoplasms after acute lymphoblastic leukemia in childhood. N Engl J Med 1991;325:1330-1336.

42. Hall EJ, Wuu CS. Radiation-induced second cancers: the impact of 3D-CRT and IMRT. Int J Radiat Oncol Biol Phys 2003;56(1):83-88.

43. Shamisa A, Bance $M, N a g$ S, et al. Glioblastoma multiforme occurring in a patient treated with gamma knife surgery. Case report and review of the literature. $J$ Ncurosurg 2002;94(5):816-21.

44. Shin M, Ueki K, Kurita H, Kirino T. Malignant transformation of a vestibular schwannoma after gamma knife radiosurgery. Lancet 2002;360:309-10.

45. Kaido $\mathrm{T}$, Hoshida $\mathrm{T}$, Uranishi $\mathrm{R}$, et al. Radiosurgery-induced brain tumor. J Neurosurg 2001;95:710-713.

46. Yu JS, Yong WH, Black KL. Glioblastoma induction after radiosurgery for meningioma. Lancet 2000;356:1576-1577.

47. LoefflerJS, Niemierko A, Chapman PH. Second tumors after radiosurgery: Tip of an iceberg or a bump in the road? Neurosurgery 2003;52(6):1436-1442.

48. Kramer BA, Wazer DE, Engler MJ, et al. Dosimetric comparison of stereotactic radiosurgery to intensity modulated radiotherapy. Radiat Oncol lnvest 1998;6:18-25.

49. Kulik C, Caudrelier JM, Vermandel M, et al. Conformal radiotherapy optimisation with micromultileaf collimators: comparison with radiosurgery techniques. IntJ Radiat Oncol Biol Phys 2002;53(4):1038-1050.

50. Ma L, Xia P, Verhey, $c t$ al. A dosimetric comparison of fan beam intensity modulated radiotherapy with gamma knife stereotactic radiosurgery for treating intermediate intracranial lesions. Int J Radiat Oncol Biol Phys 1999;45:1325-1330.

51. Mohan R, Ling C. When becometh less more? Int J Radiat Oncol Biol Phys 1995;33:235-237.

52. Söderström $\mathrm{S}$, Brahme $\mathrm{A}$. Which is the most suitable number of photon beam portals in coplanar radiation therapy? Int J Radiat Oncol Biol Phys 1995;33:151-159.

53. Stein J, Mohan R, Wang XH, ct al. Number and orientations of beams in intensity-modulated radiation treatments. Med Plys 1997;24:149-160 



\section{PART III}

\section{Clinical outcome}


CHAPTER 6

Fractionated stereotactic radiotherapy boost after post-operative radiotherapy in patients with high grade gliomas

Brigitta G. Baumert, Johannes Lutterbach, René Bernays, J. Bernard Davis and Frank L. Heppner

Radiotherapy and Oncology 2003;67(2): 183-190 


\section{Abstract}

Purpose: To determine the value and the toxicity of an additional fractionated stereotactic boost as used in the joint randomised EORTC-22972/MRC-BR10 study in patients with malignant gliomas.

Materials and methods: Seventeen patients (11 male, six female) with a high-grade glioma (two WHO III, 15 WHO IV) $\leq 4 \mathrm{~cm}$ in maximum diameter, with a good performance status (WHO $\geq 2$ ), were treated with a fractionated stereotactic radiotherapy (SRT) boost to $20 \mathrm{~Gy}$ in four fractions following partial brain irradiation to a dose of $60 \mathrm{~Gy}$ in 30 fractions. This patient group was compared with historical data in a matched-pair analysis.

Results: All patients were treated by conventional radiotherapy and a SRT boost (15 patients received $20 \mathrm{~Gy}$ and two patients $10 \mathrm{~Gy}$ ). Acute side effects included fatigue (two), impairment of short-term memory (one) and worsening of pre-existing symptoms (one). No patient developed steroid dependence after SRT. One patient was re-operated for radiation necrosis. At a median follow-up of 25 months (9-50 months) 14 patients recurred locally. Survival was $77 \%$ at 1 year and $42 \%$ at 2 years; progression-free survival was $70 \%$ at 1 year and $35 \%$ at 2 years for all patients, respectively. Median survival for the whole patient group is 20 months. Comparison with a matched historical group showed a significantly better survival for the group treated with a stereotactic boost $(\mathrm{p}<0.0001)$.

Conclusion: A fractionated stereotactic boost after standard external beam radiotherapy in selected patients with high-grade glioma is feasible and well tolerated with low toxicity. Compared to historical data survival is significantly better with an additional SRT boost. However, its effectiveness has to be proven in a randomised trial.

Keywords: Stereotactic radiotherapy; Malignant glioma; Dose escalation; Glioblastoma multiforme; Toxicity 


\section{INTRODUCTION}

High-grade gliomas are the most common of all CNS tumours. Primary treatment options include surgical resection and radiotherapy. The effectiveness of radiotherapy in terms of a survival benefit was demonstrated in prospective randomized studies $^{1,38-40}$. There is evidence of a dose response relationship with doses of $\leq 60 \mathrm{~Gy}^{1}$. However, the Radiation Therapy Oncology Group (RTOG)/Eastern Cooperative Oncology Group (ECOG) study with an additional boost of $10 \mathrm{~Gy}$ to a total dose of $70 \mathrm{~Gy}$ in a large volume did not result in further improvement of survival ${ }^{4}$. The main pattern of failure is local and local control of high-grade glioma may be achieved by increasing the dose to the tumour without increasing the dose to normal brain tissue. Therefore, stereotactic radiotherapy (SRT) was proposed for dose escalation with doses of $>70 \mathrm{~Gy}$.

Focal radiotherapy has been used in the treatment of recurrent gliomas or as a boost in the initial management with encouraging preliminary results. It was administered by interstitial brachytherapy (IRT) ${ }^{11,12,20,21}$, as $S R T^{19,30}$, as radiosurgery ${ }^{14,32,36}$ or as an integrated boost with intensity-modulated radiotherapy ${ }^{35}$. Single-dose irradiation or radiosurgery carries an increased risk of radiation necrosis ${ }^{31}$. Published data suggest that fractionated SR $T$ is associated with a lower incidence of necrosis compared with IR $T^{19,26,30}$. Fractionated SRT combines the radiobiological advantages (normal tissue sparing) and precise focal dose delivery $9,10,15$. This prospective study was conducted in order to assess the value of a fractionated stereotactic boost after conventional radiotherapy with respect to survival, local control and toxicity in the initial management of selected patients with malignant glioma.

\section{PATIENTS AND METHODS}

\section{Inclusion criteria}

For this study the same design as for the randomized phase III EORTC 22972 trial was used. Eligible patients were those with malignant high-grade glioma (WHO grade III/IV), enhancing tumours of $\leq 4 \mathrm{~cm}$ in maximum diameter on pre-operative imaging (computed tomography (CT) or magnetic resonance imaging (MRI)), age between 18 and 65 years and WHO performance status of $0-2$ (Table 1).

\section{Patient characteristics}

Between March 1997 and June 1999, 17 patients (11 male, six female) met the inclusion criteria (see Section 2.1 and Table 1). Of these, 15 had a diagnosis of glioblastoma 
Table 1. Patient characteristics

$\begin{array}{ll}\text { Patient characteristics } & \text { Details } \\ \text { Age } & 51 \text { years }(25-64.8) \\ \text { Gender } & 11 \text { male, 6 female } \\ \text { Performance Status: WHO } 0 / 1 & 5 / 12 \\ \text { Resection: } & 15 \\ \quad \text { Macroscopically total } & 2 \\ \quad \text { Residual tumour } & \\ \text { Histology: } & 2 \\ \quad \text { Anaplastic astrocytoma (WHO grade III) } & 15 \\ \text { Glioblastoma multiforme (WHO grade IV) } & \\ \text { RPA class: } & 2 \\ \text { I } & 4 \\ \text { III } & 9 \\ \text { IV } & 2 \\ \text { V } & 11 \text { months (2-49) } \\ \text { Time to recurrence } & 1 \\ \text { Necrosis } & 11 \\ \text { Number re-operations at first relapse }\end{array}$

multiforme (WHO grade IV), and two of an anaplastic astrocytoma (WHO grade III). The median age was 51 years (ranging from 25 to 65 years). All tumours were located supratentorial and were diagnosed as a unifocal contrast-enhancing lesion on CT/MR imaging. The WHO performance status was $\leq 2$ for all patients. Patient characteristics and distribution according to RTOG prognostic RPA classes ${ }^{5}$ are shown in Table 1. The definition of the prognostic classes is given in Table 2.

\section{Diagnosis}

All patients had pre-operative CT or MR scans of the brain. Histological diagnosis was obtained intra-operatively. Within $48 \mathrm{~h}$ post-operatively all patients were screened with an MRI scan for residual tumour. Pathological specimens were examined at the Institute of Neuropathology, University Hospital Zurich. The performance status was evaluated post-operatively.

\section{Treatment}

All patients were resected to a maximum safe extent with a gross total tumour resection. Complete tumour removal was confirmed by post-operative MR imaging in 15 patients (13 patients WHO grade IV, two WHO grade III). In two patients with a 
Table 2. Definition of prognostic classes (RTOG) ${ }^{2}$

(3)

I

II

III

IV

V

AA, Age $<50$, normal mental status

AA, Age $<50, \mathrm{KPS} \geq 70$, duration of symptoms $>3$ months

AA, Age $<50$, abnormal mental status, or GBM, Age $<50$, KPS $\geq 90$

AA, Age $\geq 50$, KPS $\geq 70$, duration of symptoms $\leq 3$ months, or GBM, Age $<50$, KPS $<90$, or GBM, Age $\geq 50$, KPS $\geq 70$, partial or total resection, good NF

GBM, Age $\geq 50, K P S \geq 70$, partial or total resection, unfavourable NF, or GBM, Age $\geq 50$, KPS $\geq 70$, biopsy only, or GBM or AA, Age $\geq 50$, KPS $<70$, normal mental status

GBM or AA, Age $\geq 50$, KPS $<70$, abnormal mental status

${ }^{a}$ RTOG RPA: Radiation Therapy Oncology Group, Recursive Partitioning Analysis';

bAA: Anaplastic Astrocytoma; GBM: Glioblastoma multiforme; KPS: Karnofsky Performance Status; NF: Neurological Function

glioblastoma multiforme residual tumour was found. Patients received no accompanying chemotherapy before or during radiotherapy.

Conventionally fractionated radiotherapy was given at the referring institutions. The patients were immobilized with individual thermoplastic masks. Radiotherapy treatment planning was three-dimensional. The planning target volume (PTV) for the partial brain irradiation was defined as the pre-operative tumour volume plus a margin of $2-3 \mathrm{~cm}$. A reduction of the margin to $1.5-2 \mathrm{~cm}$ after $50 \mathrm{~Gy}$ was performed only if organs-at-risk were close to the tumour. All patients were treated with three fields; each field conformed to a projection of the PTV using the beam's-eye-view feature of the treatment planning system. Fifteen patients received partial brain irradiation to a total dose of $60 \mathrm{~Gy}$ in 30 fractions, and two patients received 59.4 and $54 \mathrm{~Gy}$ in 33 and 30 fractions, respectively.

\section{SRT technique}

All patients were referred to the University Hospital Zurich for the administration of the SRT boost. The stereotactic boost was given usually within 2 weeks after the end of conventional partial brain irradiation. Head fixation was by means of a special relocatable stereotactic frame and a standardized upper jaw fixation (BrainLAB AG, Heimstetten, Germany).

A second planning CT scan with intravenous contrast was performed using a slice thickness and distance of $3 \mathrm{~mm}$. The stereotactic PTV included the pre-operative tumour volume plus a margin of $2 \mathrm{~mm}$ based on the preoperative contrast-enhancing region on $\mathrm{CT}$ or $\mathrm{T} 1$-weighted 
MR scans. If there was no residual tumour, the operation cavity plus $2 \mathrm{~mm}$ was used as the stereotactic PTV. Target volumes were defined in co-operation with the operating neurosurgeon. The margin for the stereotactic boost was defined according to the repositioning accuracy, measured to be $<2 \mathrm{~mm}$ (unpublished data: Baumert $\mathrm{B}$, Davis JB, 2001). Planning was performed using the BrainLAB treatment planning system (TPS) with BrainSCAN ${ }^{\circledR}$ software 4.03 .

The SRT boost was delivered using four to six noncoplanar static fields; each conformed to the projection of the PTV by a micro multi-leaf collimator mounted on a linear accelerator (Siemens Medical Systems, Erlangen, Germany) with a nominal beam energy of $6 \mathrm{MV}$. The mMLC used is manually operated and based on a DKFZ design (Deutsches Krebsforschungszentrum Heidelberg, Germany ${ }^{27}$ ) with two sets of 40 non-divergent tungsten leaves (with a width of $1.5 \mathrm{~mm}$ at the isocentre).

The dose was prescribed at the isocentre according to ICRU 5017. However, for SRT ICRU dose prescriptions cannot always be fully followed. When this was not possible, the $90 \%$ isodose was accepted as the minimum target dose. Two patients received a boost dose of $10 \mathrm{~Gy}$ in two fractions and 15 patients a total boost dose of 20 $\mathrm{Gy}$ in five fractions. The optic apparatus and the brain stem received doses of less than 50 or $60 \mathrm{~Gy}$, respectively. Prophylactically, patients received daily doses of $8 \mathrm{mg}$ corticosteroids for 4-7 days, starting with the first fraction of the SRT boost.

\section{Follow-up and definition of relapse}

Patients were seen weekly during radiotherapy and after the last fraction of the stereotactic boost treatment. The first follow-up examination was done 2 months after conventional radiotherapy. Thereafter patients were seen every 3 months during the first year after treatment, every 6 months in the second year and subsequently at yearly intervals or as necessary. Radiological follow-up was performed with an MRI scan, for the first time 3 months after conventional radiotherapy, thereafter in yearly intervals or if clinical progression was seen. Physical examination included a complete neurological status. Additional scans and clinical assessments were performed if new symptoms developed or deterioration was observed.

Progression-free survival and overall survival were measured from the start of conventional radiotherapy. Progression was defined as neurological progression or as radiological progression, whichever occurred first. Neurological progression was defined as the occurrence of new symptoms or a progression of pre-existing symptoms not responding to steroids; radiological progression was defined as a new contrast-enhancing lesion or an increase in size in two consecutive follow-up scans compared to the previous imaging. 


\section{Historical data comparison}

For historical comparison, we used a database of the Department of Radiotherapy, University of Freiburg, Germany. From 1980 to 2000, 209 patients with a glioblastoma underwent accelerated hyperfractionated radiotherapy at this hospital. All patients had a gross total resection. Details of the protocol have been published previously 22 . In summary, three daily fractions of $1.5 \mathrm{~Gy}$ were administered to a total dose of $54 \mathrm{~Gy}$. The treatment was started as whole brain radiotherapy. After $31.5 \mathrm{~Gy}$, the PTV was reduced to the contrast-enhancing lesion plus a $3 \mathrm{~cm}$ margin.

The 15 patients with the diagnosis of a glioblastoma multiforme (WHO grade IV) from Zurich were compared with 30 patients from Freiburg derived from the above-mentioned database and matched for the variables age, sex, tumour size and performance status (WHO 0-2).

\section{Statistical analysis}

Survival probabilities were calculated using the method of Kaplan and Meier. Survival curves were compared using the log-rank test. The analysis was done with StatView software (version 5.0.1, 1998). Results were considered to be significant if $p$-values were $<0.05$ using the log-rank test for comparison.

\section{RESULTS}

\section{Overall survival}

The median follow-up was 25 months (range 9-50 months). Twelve patients had died and five patients were still alive at the time of the analysis. The median overall survival for all patients is 20 months, with a survival of $77 \%, 42 \%, 28 \%$, and $22 \%$ at 1 , 2,3 , and 4 years, respectively (Fig. 1). The overall and progression-free survival for the 15 patients with the diagnosis of a glioblastoma multiforme (WHO grade IV) were not significantly different: the median survival was 19 months. Survival according to RTOG-RPA prognostic classes is shown in Table 3.

\section{Local control}

At the time of the analysis 14 patients had recurred locally, six patients had a local recurrence in the first year, five patients in the second year and three patients between 2.5 and 4.2 years after radiotherapy. At the time of analysis, three patients were still tumour-free: two patients with an anaplastic astrocytoma (after 30 and 50 months 


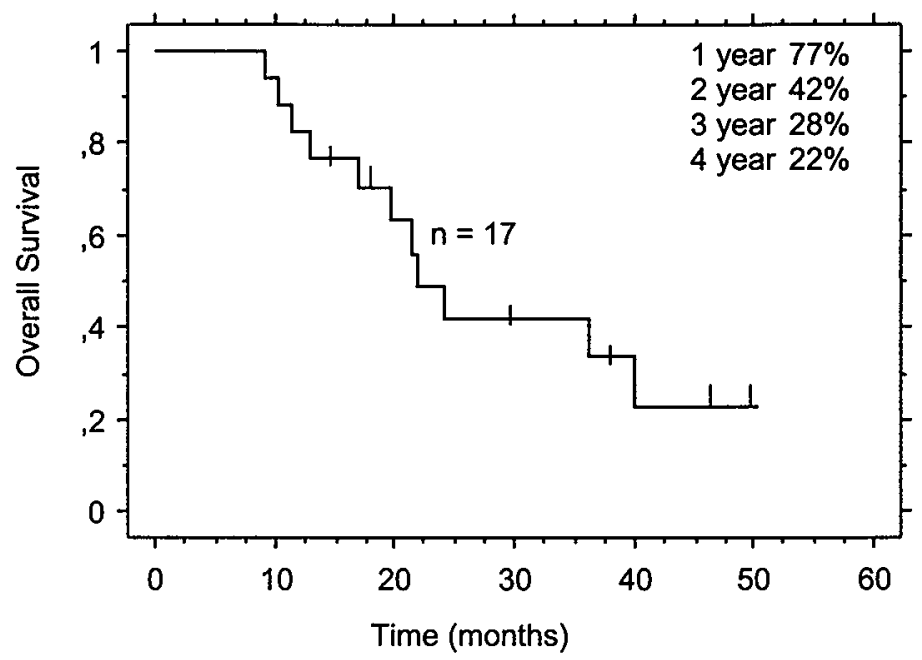

Figure 1. Actuarial overall survival of all patients with malignant gliomas (anaplastic astrocytoma $\mathrm{n}=2$ and glioblastoma multifome $\mathrm{n}=15$ ).

Table 3 Patient distribution according RTOG-RPA prognostic classes.

\begin{tabular}{lllll}
$\begin{array}{l}\text { Prognostic } \\
\text { class }\end{array}$ & No. & $\begin{array}{l}\text { Time to progression } \\
\text { (months) }\end{array}$ & $\begin{array}{l}\text { Median survival this } \\
\text { study (months) }\end{array}$ & $\begin{array}{l}\text { Median survival } \\
\text { RTOG-RPA }\end{array}$ \\
I & 2 & (Not defined yet) & 49,28 & $40-60$ \\
III & 4 & $6,2,-, 8$ & $22(11-35)$ & $11-18$ \\
IV & 9 & m $10(2-49)$ & $18(7-49)$ & $11-18$ \\
V & 2 & 9,11 & 19,14 & $5-9$ \\
\hline
\end{tabular}

a RTOG RPA: Radiation Therapy Oncology Group, Recursive Partitioning Analysis ${ }^{5}$

follow-up) and one patient with a glioblastoma multiforme (37 months follow-up). The mean time to progression after SR T boost was 11 months (range 2-49 months). Progression-free survival was $70 \%, 35 \%$, and $23 \%$ at 1,2 , and 3 years, respectively (Fig. 2).

\section{Treatment at relapse}

At the time of relapse different therapies were used: reoperation, chemotherapy with temozolomide, thalidomide, re-irradiation with a stereotactically guided boost (one patient) or other experimental treatments. Nine patients were re-operated at the time of suspected relapse, two patients several times. Thirteen of 15 patients with a glioblastoma multiforme (WHO grade IV) received a therapy with temozolomide and thalidomide at the time of recurrence. 


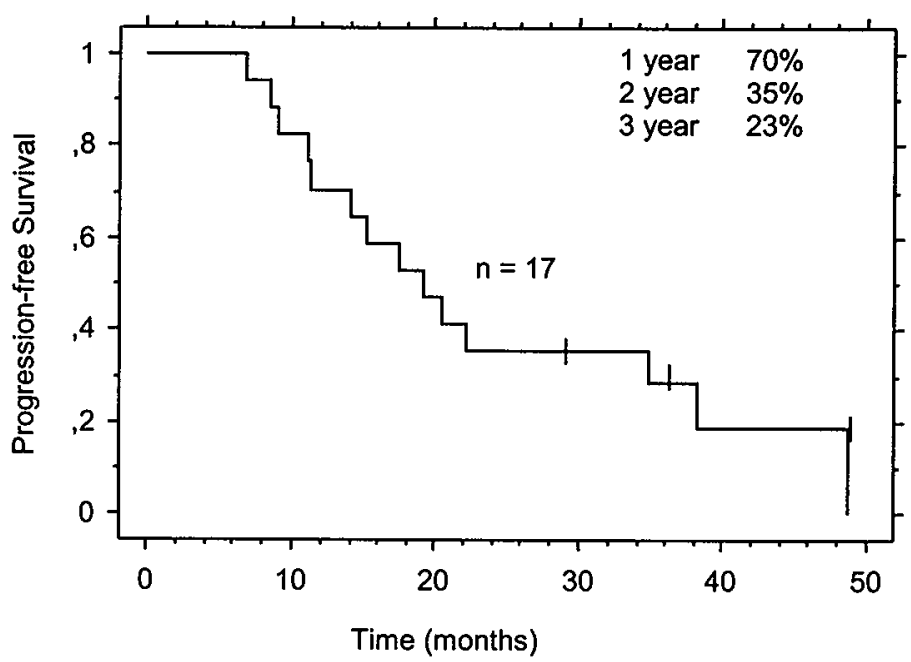

Figure 2. Progression-free survival of all patients with malignant gliomas (anaplastic astrocytoma $n=2$ and glioblastoma multifome $n=15$ ).

\section{Toxicity}

The additional stereotactic boost was associated with little acute toxicity. Acute side effects included fatigue (two patients) and worsening of pre-existing symptoms (one). The latter patient had a transient deterioration of a paresis, which responded to steroids. No patient developed steroid dependence after SRT. One patient had worsening of pre-existing impairment of short-term memory, which had started already after post-operative radiotherapy.

One patient suffering from progressive hemianopsia was re-operated due to a suspected relapse. MR imaging showed a new contrast-enhancing area 8 months after radiotherapy. However, histological examination revealed radiation necrosis and no viable tumour cells were found, although it cannot be formally excluded that no tumour was found due to a sampling error. This seems to be likely, since the patient suffered from a tumour recurrence 3 months later, which was confirmed histologically at re-operation.

\section{Matched-pair analysis}

The results of the matched-pair analysis for patients with the diagnosis of a glioblastoma multiforme (WHO grade IV) only are shown in Fig. 3. The difference in survival between the two patient groups was statistically significant $(p<0.0001)$. In two patient groups with the same inclusion criteria survival was better for patients who received a stereotactic boost. 


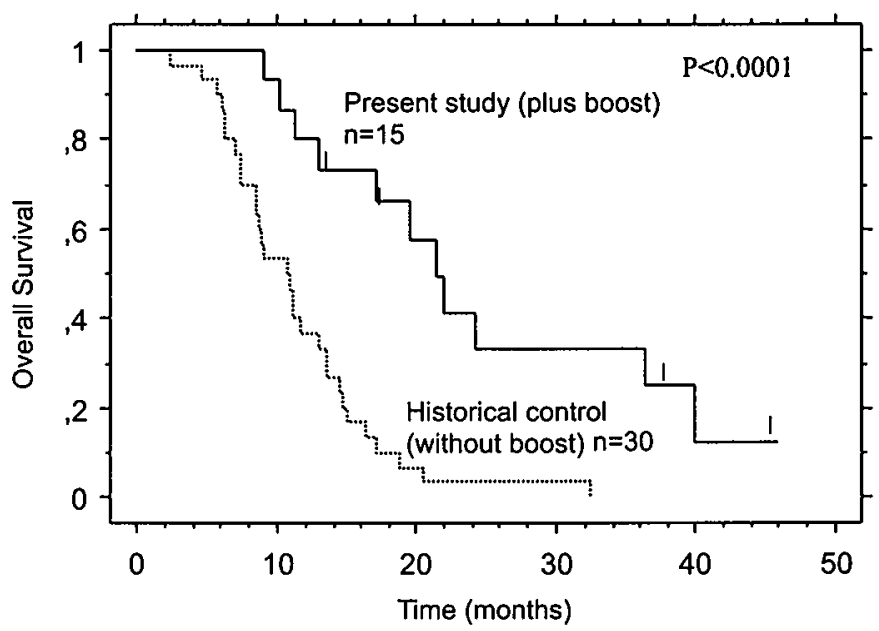

Figure 3. Matched Pair Analysis (2:1): Comparison with historical group for overall survival of patients with a glioblastoma multiforme.

\section{DISCUSSION}

Compared with historical reports, the combination of single fraction radiosurgery (SRS) and conventional external beam radiotherapy in the initial management of patients with malignant gliomas has not conclusively proved to result in an increase in survival. The increase in survival which has been shown in studies using an interstitial radiotherapy (IRT) boost is supposed to be mainly the result of patient selection ${ }^{7}$. In phase II studies with an additional IRT boost ${ }^{12,21,41}$ the reported median survival time of 27 months was reduced to 18 months after a longer follow-up. If data are adjusted according to RTOG prognostic classes, only an improvement for the poorer prognostic classes was seen ${ }^{37}$.

Published retrospective studies of patients managed with a radiosurgical boost with doses from 10 to $35 \mathrm{~Gy}$ as part of their primary treatment have shown a median survival of 9-25 months for patients with the diagnosis of glioblastoma multiforme (WHO grade IV) and 14-56 months for patients with an anaplastic astrocytoma (WHO grade III) (see Table 4). In another study in which a proton boost of 25-30 Gy was used for dose escalation, the median survival was 20 months $^{6}$, the same as in this study. Again, differences in survival seem to be due to selection: a multi-centre analysis with comparison to the RTOG recursive partitioning analysis showed a broad variation in the distribution of prognostic criteria between different centres ${ }^{26}$. 
Patients who are most likely to benefit from an additional stereotactic boost are often pre-selected; thus, they belong to a better prognostic group. Variation in survival is predominantly related to selection criteria such as age, tumour volume, histology, performance status, extent of surgery and primary tumour site, which have been identified as prognostic factors $5,28,33,34$. The non-parametric recursive partitioning analysis based on three RTOG trials stratified patients into six prognostic classes with different survival ${ }^{5}$ (Table 2), which also holds for the current study. It is unknown whether a smaller tumour size is associated with a more favourable prognosis. Small tumours are selected for nearly all dose escalation studies using a stereotactic boost for reasons of toxicity and technical feasibility (Table 4). For the comparison with the historical control group we have included tumour size as a matching variable.

In the matched-pair analysis the significantly better survival of the patients with the diagnosis of a glioblastoma multiforme (WHO grade IV) treated with a stereotactic boost appears not to be related to different prognostic groups: the survival advantage was maintained in the poorer prognostic group if compared to median survival of the RTOG recursive partitioning analysis (Table 3 ). There are differences in radiotherapy treatment within the two groups (different institutions, total dose, fractionation, treatment technique, 2D and 3D planning), but it is not expected that these will have an influence on overall survival as demonstrated in a recent population-based cohort study ${ }^{25}$. Another small study has shown a significantly better survival for patients treated with a radiosurgery boost compared to patients treated with standard radiotherapy alone $e^{24}$.

Fractionated SRT is associated with a lower incidence of necrosis. This has been shown in a dose escalation study escalating the total dose from 20 to 50 Gy using 5.0 Gy fractions in patients re-irradiated with SRT at the time of recurrence ${ }^{19,30}$. The actuarial risk of developing presumed delayed radiation side effects was reported to be as high as $34 \%$. A SRT dose of $>40$ Gy was a significant predictor of radiation toxicity ${ }^{19}$. Another study using fractionated SRT for patients with recurrent gliomas, escalating to $35 \mathrm{~Gy}$ in $3.5 \mathrm{~Gy}$ fractions, reported no toxicity ${ }^{16}$, whereas all patients with a total dose of $90 \mathrm{~Gy}$ developed radiation necrosis ${ }^{6}$. For total doses ranging from 75 to $90 \mathrm{~Gy}$, necrosis rates of between 3 and $33 \%$ were reported ${ }^{18,26,29}$ (see Table 4). The highest necrosis rate was described in patients treated with an accelerated scheme and a concomitant stereotactic boost $(3 \times 12 \mathrm{~Gy})^{3}$. In radiosurgery, the dose is usually prescribed to the margin and not to the isocentre as in our study, thus resulting in higher central doses. Therefore, a comparison of boost doses and the associated toxicity is difficult as the reported doses are different. However, in our study only for one out of 17 patients was a radiation necrosis observed, thus suggesting a fractionated stereotactic boost scheme to a dose of 20 Gy to be safe. 


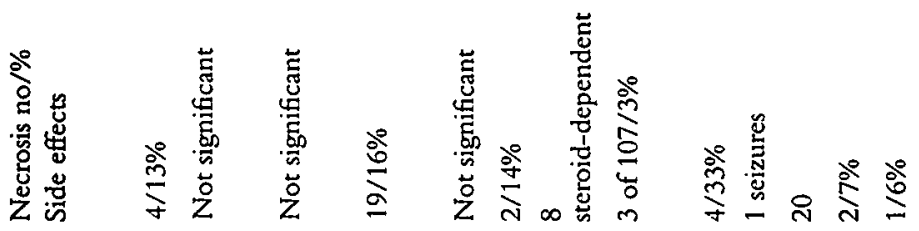
㞼

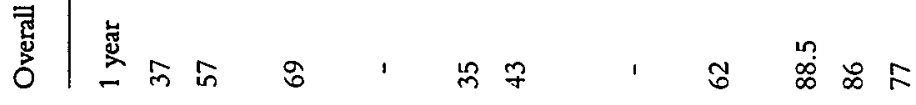

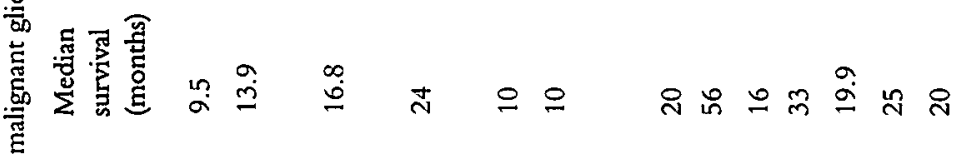
萦

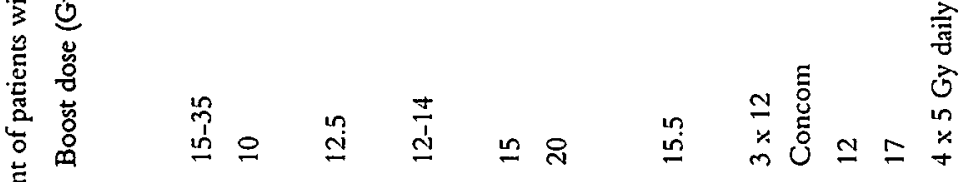
总 窎 苛

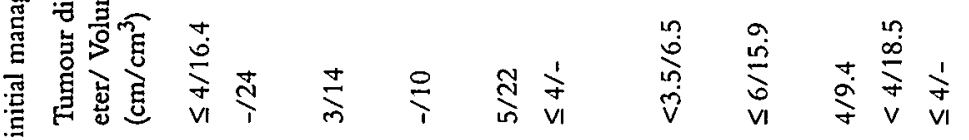
营

岁 壳

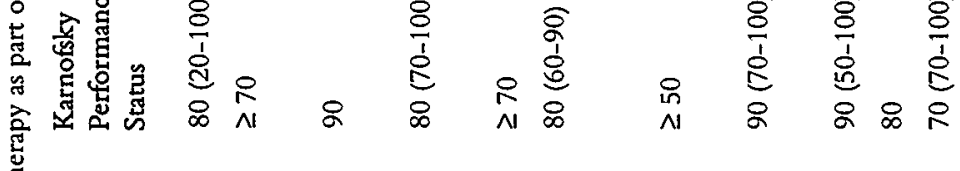
产

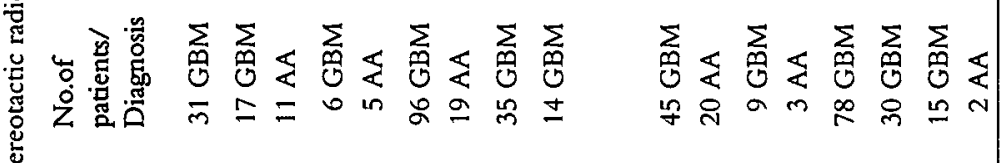
总

E

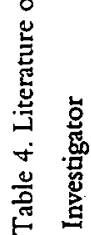

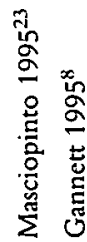

芯

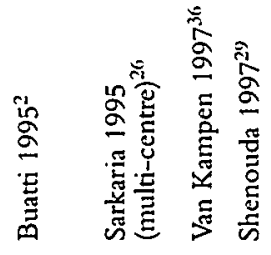

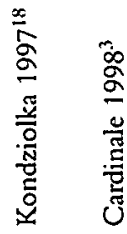

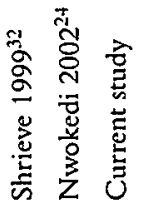


Selection criteria could be the reason for prolonged median survival in many retrospective studies and selected control groups ${ }^{13}$. However, this small study suggests a survival advantage for patients who received a stereotactic boost of $20 \mathrm{~Gy}$. We are aware that a retrospective comparison with a historical group cannot definitely answer the question of a survival advantage of an additional stereotactic boost. The role of a stereotactic boost in the initial management of malignant gliomas remains to be defined by prospective randomized studies.

\section{REFERENCES}

1. Bleehen NM, Stenning SP. A Medical Cancer Research Council trial of two radiotherapy doses in the treatment of grades 3 and 4 astrocytoma. The Medical Research Council Brain Tumour Working Party. Br J Cancer 1991;64:769-74.

2. Buatti JM, Friedman WA, Bova FJ, Mendenhall WM. Linac radiosurgery for high-grade gliomas: the University of Florida experience. Int J Radiat Oncol Biol Phys 1995;32:205-10.

3. Cardinale RM, Schmidt-Ullrich RK, BenedictSH, Zwicker RD, Han DC, Broaddus WC. Accelerated radiotherapy regimen for malignant gliomas using stereotactic concomitant boosts for dose escalation. Radiat Oncol Invest 1998;6:175-81.

4. Chang $\mathrm{CH}$, Horton J, Schoenfeld D, et al. Comparison of postoperative radiotherapy and combined postoperative radiotherapy and chemotherapy in the multidisciplinary management of malignant gliomas. Cancer 1983;52:997-1007.

5. Curran WJ, Scott CB, Horton J, et al. Recursive partitioning analysis of prognostic factors in three Radiation Therapy Oncology Group malignant glioma trials. J Natl Cancer Inst 1993;85:704-10.

6. Fitzek MM, Thornton AF, Rabinov JD, et al. Accelerated fractionated proton/photon irradiation to 90 cobalt gray equivalent for glioblastoma multiforme: results of a phase II prospective trial. J Neurosurg 1999;91:251-60.

7. Florell RC, MacDonald DR, Irish WD, et al. Selection bias, survival, and brachytherapy for glioma. J Neurosurg 1992;76:179-83.

8. Gannett D, Stea B, Lulu B, Adair T, Verdi C, Hamilton A. Stereotactic radiosurgery as an adjunct to surgery and external beam radiotherapy in the treatment of patients with malignant gliomas. Int J Radiat Oncol Biol Phys 1995;33:461-8.

9. Gill SS, Thomas DG, Warrington AP, Brada M. Relocatable frame for stereotactic external beam radiotherapy. Int J Radiat Oncol Biol Phys 1991;20:559-603.

10. Graham JD, Warrington AP, Gill SS, Brada M. A non-invasive, relocatable stereotactic frame for fractionated radiotherapy and multiple imaging. Radiother Oncol 1991;21:60-2.

11. Gutin $P$, Leibel $S$, Wara W, et al. Recurrent malignant gliomas: survival following interstitial brachytherapy with high-activity iodine-125 sources. J Neurosurg 1987;67:864-73. 
12. Gutin PH, Prados MD, Phillips TL, ct al. External irradiation followed by an interstitial high activity iodine-125 implant "boost" in the initial treatment of malignant gliomas: NCOG study 6G-82-2. Int J Radiat Oncol Biol Phys 1991;21:601-6.

13. Haines SJ. Moving targets and ghosts of the past: outcome measurement in brain tumour therapy. J Clin Neurosci 2002;9:109-12.

14. Hall WA, Djalilian HR, Sperduto PW, et al. Stereotactic radiosurgery for recurrent malignant gliomas. J Clin Oncol 1995;13:1642-8.

15. Hariz MI, Henriksson R, Lofroth PO, Laitinen LV, Saterborg NE. A non-invasive method for fractionated stereotactic irradiation of brain tumours with linear accelerator. Radiother Oncol 1990;17:57-72.

16. Hudes RS, Corn BW, Werner-Wasik M, et al. A phase I dose escalation study of hypofractionated stereotactic radiotherapy as salvage therapy for persistent or recurrent malignant glioma. Int J Radiat Oncol Biol Phys 1999;43:293-8.

17. ICRU, ICRU Report 50. Prescribing, recording, and reporting photon beam therapy. Bethesda, MD: International Commission on Radiation Units and Measurements; 1993.

18. Kondziolka D, Flickinger JC, Bissonetter DJ, Bozik M, Lunsford LD. Survival benefit of stereotactic radiosurgery for patients with malignant glial neoplasms. Neurosurgery 1997; 41:776-83.

19. Laing RW, Warrington AP, Graham J, Britton J, Hines F, Brada M. Efficacy and toxicity of fractionated stereotactic radiotherapy in the treatment of recurrent gliomas (phase I/II study). Radiother Oncol 1993;27:22-9.

20. Leibel SA, Gutin PH, Wara WM, et al. Survival and quality of life after interstitial implantation of removable high-activity Iodine-125 sources for the treatment of patients with recurrent malignant gliomas. Int J Radiat Oncol Biol Phys 1989;17:1129-39.

21. Loeffler J, Alexander III E, Wen PY, et al. Results of stereotactic brachytherapy used in the initial management of patients with glioblastoma. J Natl Cancer Inst 1990;82:1918-21.

22. Lutterbach J, Weigel P, Guttenberger R, Hinkelbein W. Accelerated hyperfractionated radiotherapy in 149 patients with glioblastoma multiforme. Radiother Oncol 1999; 53:49-52.

23. Masciopinto JE, Levin AB, Mehta MP, Rhode BS. Stereotactic radiosurgery for glioblastoma: a final report of 31 patients. J Neurosurgery 1995;82:530-5.

24. Nwokedi EC, DiBiase SJ, Jabbour S, ct al. Gamma knife stereotactic radiosurgery for patients with glioblastoma multiforme. Neurosurgery 2002;50(1):41-6.

25. Paszat L, Laperriere N, Groome P, Schulze K, Mackillop W, Holowaty E. A population-based study of glioblastoma multiforme. Int J Radiat Oncol Biol Phys 2001;51:100-7.

26. Sarkaria JN, Mehta MP, Loeffler JS, ct al. Radiosurgery in the initial management of malignant gliomas: survival comparison with the RTOG recursive partitioning analysis. Int J Radiat Oncol Biol Phys 1995;32:931-41.

27. Schlegel W, Pastyr O, Bortfeld $T$, et al. Stereotactically guided fractionated radiotherapy: technical aspects. Radiother Oncol 1993; 29:197-204.

28. Sheline GE. Radiotherapy for high grade gliomas. Int J Radiat Oncol Biol Phys 1991; 18:793-803. 
29. Shenouda G, Souhami L, Podgosark EB, ct al. Radiosurgery and accelerated radiotherapy for patients with glioblastoma. Can J Neurol Sci 1997;24:110-5.

30. Shepherd SF, Laing RW, Cosgrove VP, ct al. Hypofractionated stereotactic radiotherapy in the management of recurrent glioma. Int J Radiat Oncol Biol Phys 1997;37:393-8.

31. Shrieve DC, Alexander 3rd E, Wen PY, et al. Comparison of stereotactic radiosurgery and brachytherapy in the treatment of recurrent glioblastoma multiforme. Neurosurgery 1995; 36:275-84.

32. Shrieve DC, Alexander 3rd E, Black PM, et al. Treatment of patients with primary glioblastoma multiforme with standard postoperative radiotherapy and radiosurgical boost: prognostic factors and longterm outcome. J Neurosurg 1999;90:72-7.

33. Simpson JR, Horton J, Scott C, et al. Influence of location and extent of surgical resection on survival of patients with glioblastoma multiforme: results in three consecutive Radiation Therapy Oncology Group (RTOG) clinical trials. Int J Radiat Oncol Biol Phys 1993; 26:239-44.

34. The Medical Research Council Brain Tumour Working Party, Prognostic factors for high-grade malignant glioma: development of a prognostic index. A report. J Neurooncol 1990;9:47-55.

35. Thilmann C, Zabel A, Grosser KH, Hoess A, Wannenmacher M, Debus J. Intensity-modulated radiotherapy with an integrated boost to the macroscopic tumour volume in the treatment of high-grade gliomas. Int J Cancer 2002;96:341-9.

36. Van Kampen M, Engenhart-Cabillic R, Debus J, Fuss M, Rhein B, Wannenmacher $M$. Stellenwert der Radiochirurgie in der Primaertherapie des Glioblastoma multiforme. Strahlenther Onkol 1998;174: 187-92.

37. Videtic GMM, Gaspar LE, Zamorano L, et al. Use of the RTOG recursive partitioning analysis to validate the benefit of iodine- 125 implants in the primary treatment of malignant gliomas. Int J Radiat Oncol Biol Phys 1999;45:687-92.

38. Walker MD, Strike TA, Sheline GE. An analysis of dose-effect relationship in the radiotherapy of malignant gliomas. Int J Radiat Oncol Biol Phys 1979;5:1725-31.

39. Walker MD, Green SB, Byar DP, et al. Randomized comparisons of radiotherapy and nitrosoureas for the treatment of malignant glioma after surgery. N Engl J Med 1980;303: 1323-9.

40. Walker MD, Strike TA, Sheline GE. Evaluation of BCNU and/or radiotherapy in the treatment of anaplastic gliomas. A cooperative clinical trial. J Neurosurg 1978;49:333-43.

41. Wen PY, Alexander III E, Black PM, et al. Long term results of stereotactic brachytherapy used in the initial treatment of patients with glioblastomas. Cancer 1994;73:3029-36. 


\section{CHAPTER 7}

\section{Early improvements in vision after fractionated stereotactic radiotherapy for primary optic nerve sheath meningioma}

Brigitta G. Baumert, Salvador Villà, Gabriela Studer, René-Olivier Mirimanoff, J. Bernard Davis, Klara Landau, Nicolas Ducrey, Jordi Arruga, Philippe Lambin and Alessia Pica

Radiotherapy and Oncology 2004;72(2):169-174 


\begin{abstract}
Backgromind and purpose: To report on the efficacy and follow-up of 23 patients with primary optic nerve sheath meningioma (ONSM) with fractionated stereotactic conformal radiotherapy (SCRT).

Patients and Methods: Between 1996 and 2003, 23 patients (= 23 eyes) with ONSM were treated. Indications for primary stereotactic radiotherapy were tumour progression documented by imaging or symptoms (loss of vision, pain). All patients received SCRT with a median dose of 50.4 Gy in 6 weeks.

Results: After a median follow-up of 20 months (1-68 months) a 95\% (21 of 22 ) visual control was seen: vision improved in 16 patients and remained stable in 5. For 13/16 patients improvement was documented already within 1-3 months after SCRT. Vision became worse in one patient. An improvement of pain was observed after radiotherapy in 6 patients as well as of proptosis in 1 patient. For 1 patient pain was persistent after SCRT. In one patient 4 years after SCRT a radiation retinitis and vitreous haemorrhage was seen.

Conclusions: Fractionated stereotactic radiotherapy improves vision, often shortly after treatment, and is thus a viable treatment option for this tumour entity.
\end{abstract}

Keyuords: Stereotactic radiotherapy, optic nerve, meningioma, orbit, optic nerve sheath, organs at risk, vision 


\section{INTRODUCTION}

Optic nerve sheath meningiomas (ONSM) are primary tumours of the optic nerve sheath and are a different tumour entity than optic nerve gliomas. Primary orbital meningiomas are very rare: they occur in 1-2\% of all meningiomas and in $35 \%$ of all optic nerve tumours. They can originate in the orbit (primary OSNM) or grow into the orbit from an intra-cranial lesion (secondary ONSM). Characteristically, their natural history is that of a slow indolent pattern of growth resulting in gradual progressive and unremitting loss of vision. Proptosis, motility disturbance, progressive visual loss and lid oedema are the leading symptoms ${ }^{2,5}$.

The optimal management still remains controversial. Until recently, a conservative approach based on observation has been the preferred option until a worsening of vision is documented. Other treatment options reported in literature are radiotherapy and surgical interventions, the latter often leading to visual deterioration or total visual loss often due to a central artery occlusion ${ }^{5,12,13}$. Furthermore, a high rate of local recurrence after operation has been observed ${ }^{4}$. Thus, because of these reasons and the often incomplete removal of the tumour, surgery should be reserved for selected cases, e.g. for severe proptosis.

Older studies using conventional radiotherapy and a more recent study with long-term follow-up ${ }^{18,24,26}$ demonstrated, that the best visual outcome was obtained for patients receiving radiotherapy alone. Newer treatment techniques, which are able to deliver a highly conformal treatment irradiating only the meningioma of the optic nerve, can spare critical normal tissues. First publications using fractionated stereotactic radiotherapy are encouraging, reporting an overall improvement in vision between $40^{1,23}$ and $80 \%{ }^{17}$. Several case reports using either fractionated stereotactic radiotherapy (SCRT) or other conformal radiation techniques (3D conformal, intensity modulation) showed the same good responses ${ }^{7,10,14,15,19}$ with no or very little toxicity so far. Thus, with this study, we wanted to assess the efficacy of treatment in terms of functional outcome for patients with primary ONSM.

\section{PATIENTS AND METHODS}

\section{Indication for stereotactic radiotherapy}

Patients with an ONSM primarily originating from the optic nerve qualified for the study. Patients were referred after multi-disciplinary discussion, several at the time of progression (clinical progression and/or on imaging). Twenty-two patients had an ophthalmologically documented progressive visual deterioration. For two patients, 
treatment was recommended in order to prevent further growth of the tumour to the contra-lateral optic nerve (cases 3 and 23). For case 9 the main indication beside the tumour extension was worsening of vision. Seven patients had orbital pain due to the meningioma, and for 6 patients proptosis of the involved eye was an additional indication for treatment.

\section{Patient characteristics}

Between 1996 and 2003, 23 patients, each with one eye involved ( 4 male, 19 female) were referred from four ophthalmologic departments for stereotactic radiotherapy to the departments of Radiation Oncology of the University Hospital Zurich (USZ, 9 cases), Institut Català d'Oncologia (ICO, 6 cases), the University Hospital Lausanne (CHUV, 7 cases) and University Hospital Maastricht (MAASTRO, 1 case). All patients received fractionated stereotactic conformal radiotherapy (SCRT). The median age was 44 years $(9-71$ years) at the time of irradiation. Two patients (cases 4 and 20) had a bilateral ONSM, of which only the symptomatic tumour site was treated. One patient was already blind in the affected eye for several years at the time of referral (case 3), and one patient was nearly blind (case 23). The WHO performance status was $0-1$ for all patients.

\section{Diagnosis}

Diagnosis was based mainly on diagnostic imaging and ophthalmologic examination, as histologic biopsies did not belong to the standard work-up due to the high risk of side effects. The use of specified criteria and high quality imaging allows the diagnosis to be made without tissue biopsy ${ }^{5,13}$. All patients had MR and CT scans of the orbit. Scans were screened for the presence of typical diagnostic criteria as the "tram-track-sign", isodensity of the tumour compared to noral brain tissue and contrast enhancement. One patient (case 12) had a partial resection before SCRT and the child (case 20) a biopsy. In both cases a histological diagnosis of a meningothelial meningioma WHO I was confirmed.

\section{Stereotactic radiotherapy treatment}

All patients were immobilized and scanned in a relocatable stereotactic frame (BrainLAB AG, Heimstetten, Germany) and had a planning CT using a slice thickness of $1-3 \mathrm{~mm}$. All but 2 patients had an additional individually customized bite-block. All patients from ICO, MAASTRO, CHUV and patients treated at USZ after 2000 had additional MRI scans for tumour localisation, which were fused to the $\mathrm{CT}$ scan for planning. After transferring the CT data, target and non-target volumes i.e., the region of contrast enhancement on $\mathrm{CT}$ and organs-at-risk (OAR) were 
delineated in all relevant slices, using the BrainLAB treatment planning system (TPS) with BrainSCAN ${ }^{\circledR}$ software (versions 3.53, 3.5B, 4.2, classic 4.03 and XL 5.1).

The gross tumour volume (GTV) was defined as the contrast-enhancing lesion on $\mathrm{CT}$ and MRI scans. For planning T1 weighted MR images with gadolinium, which are co-registered to the $\mathrm{CT}$ scan, were used. No additional margin for clinical target volume (CTV) was used, as benign meningioma do not infiltrate into surrounding structures. Thus, the CTV is the same as the GTV. For the planning target volume (PTV) a margin of $2-3 \mathrm{~mm}$ (for the definition of the $95 \%$ isodose line) was added to the GTV three-dimensionally. The median volumes of the GTV were $1.7 \mathrm{~cm}^{3}(0.46$ $-26.3 \mathrm{~cm}^{3}$ ). All tumours were located close to an OAR. In the case of close proximity of tumour and retina a smaller margin was accepted. No additional device to reduce eye movements was used, because the movements were already restricted by the optic meningioma itself. SCRT was delivered using 3 - 5 non-coplanar static fields, each conformed to the projection of the PTV by a micro-multileaf collimator (mMLC) or individual cerrobend blocks mounted on a linear accelerator with nominal beam energy $6 \mathrm{MV}$. All treatment plans used one single isocentre. The dose was prescribed at the isocentre according to ICRU 50 recommendations ${ }^{11}$. Total prescribed dose was $45-54 \mathrm{~Gy}$ in fractions of $1.8-2.0 \mathrm{~Gy}$, median $50.4 \mathrm{~Gy}$, given within 6 weeks. For one patient (case 6 ) the fraction size was reduced to $1.7 \mathrm{~Gy}$ due to a very close proximity of the lesion to the retina. Dose restrictions used were lower than usual as ONSM are benign tumours. For the contralateral optic nerve and the pituitary, the dose tolerated was the minimum possible, for the chiasm the minimum possible but not more than $50 \mathrm{~Gy}$, for the lens $<5 \mathrm{~Gy}$, for the ipsi-lateral retina and contra-lateral retina $<36-45 \mathrm{~Gy}$, and less than $30 \mathrm{~Gy}$ for the lacrimal gland ${ }^{6,25}$. No prophylactic corticosteroid medication was given for patients treated before and during SCRT.

\section{Follow-up}

Patients were seen weekly during radiotherapy and $4-6$ weeks after the last fraction. All patients underwent regular ophthalmologic control with examination of the visual fields and visual acuity. The first ophthalmologic and radiation oncology follow-up examination was performed 3 months after treatment. Thereafter, patients were followed six-monthly with clinical and ophthalmologic examination. Radiological follow-up was performed with an MRI scan at least once a year. Response to treatment was defined clinically by improvement of vision or symptoms, by regular visual field (by perimetry) and visual acuity measurements (by Snellen lines) as well as by imaging with MRI scans of the orbit compared to vision before the start of SCRT. An improvement of vision was defined as a gain of more than 1 Snellen line. If vision was worse than 0.1 , visual acuity was graded as counting fingers (CF), hand motions 
(HM), light perception (LP) or no light perception (NPL). Progression of disease was defined as visual deterioration or as radiological progression. Visual deterioration was defined ophthalmologically using perimetry and Snellen lines. Radiological progression was defined as an increase in tumour size in two consecutive follow-up scans compared to the imaging before the start of radiotherapy.

\section{RESULTS}

\section{Vision and symptoms}

After a median follow-up of 20 months (range $1-68$ months) 16 of 22 eyes with documented visual decrease before SCRT improved in vision, 5 remained stable. One patient had continuous visual loss after irradiation (case 1). One patient deteriorated after 4 years with stable vision (case 13). Visual improvement was documented for 13 patients already 1-3 months after treatment. For 3 patients (cases 2, 8 and 9) visual improvement was already reported during radiotherapy (subjective observation) and documented by ophthalmologic examination 3 months after SCRT (Table 1). For 8 patients visual improvement consisted of an increase in visual acuity of more than two Snellen lines, for one patient (case 23) this was an improvement from LP to HM. In the whole group visual acuity improved for 15 patients, visual fields for 8 patients. Visual acuity as well as visual field improvement was documented for 7 patients. Visual fields showed no change in 11 patients.

For 3 of 7 patients having pain due to the tumour, the pain decreased during radiotherapy, for 4 patients $1-3$ months after SCRT. One patient out of 6 with a proptosis experienced a decrease of proptosis, which was documented on imaging with a tumour decrease (case 22). On imaging, the meningioma remained stable in almost all patients, one decreased and none increased in size. In one case, the uptake of contrast media slightly decreased (case 5)(Table 1).

\section{Sparing of organs-at-risk}

The chiasm received between 10 and $40 \%$ of the total dose in 10 out of 22 cases. The contralateral optic nerve and pituitary received doses $<30 \%$ in all 14 cases reported. Figure 1 shows the isodose distribution of a patient where the dose to the retina could be kept low. The ipsilateral retina was spared in the 9 cases with a more posterior location of the tumour, which did not reach the eye ball, with a maximum dose $<40 \mathrm{~Gy}$. 


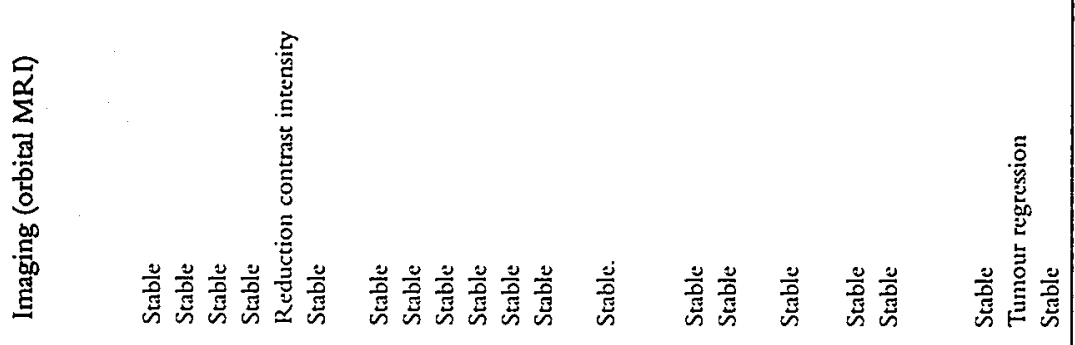

결

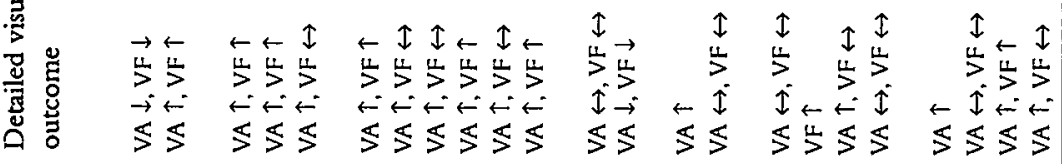

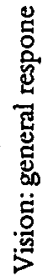
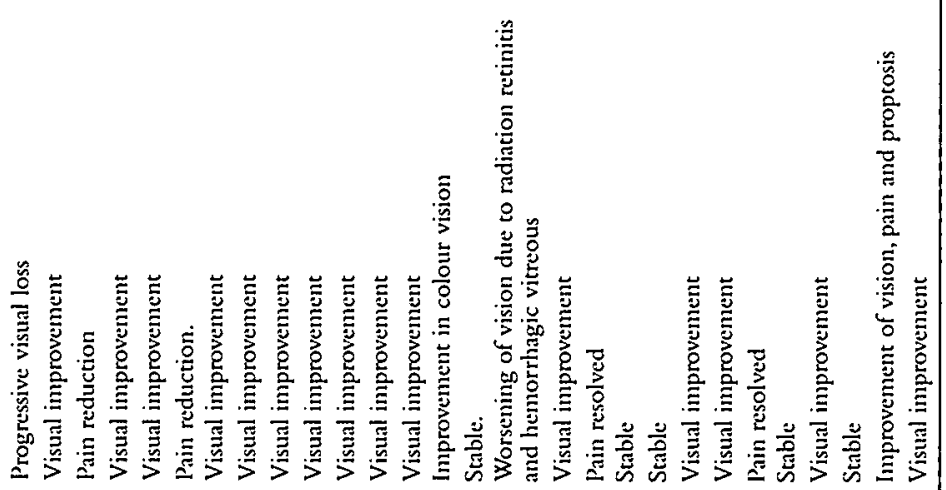

劳

둥

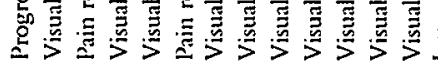

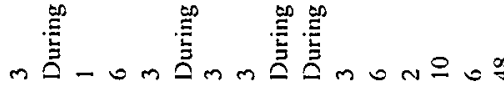

吾

言 总 


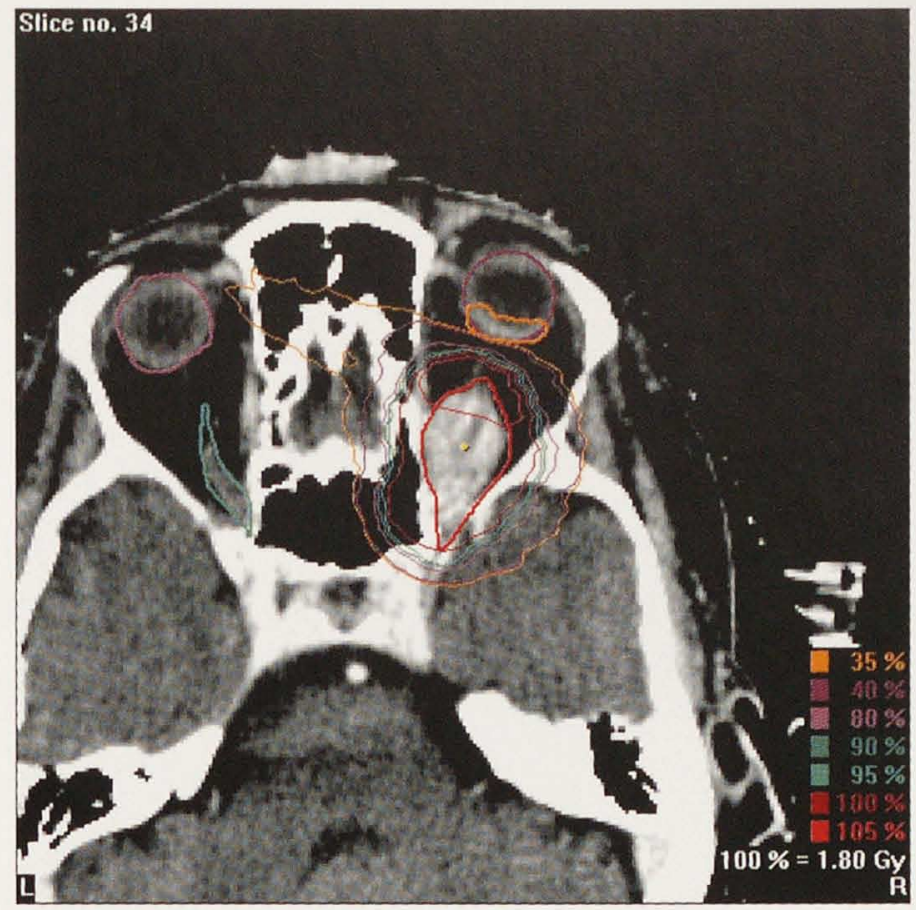

Figure 1. Case example of isodose distributions. The area of the retina is defined in orange-yellow. Note the steep dose fall-off between the 95 (light green) and 35\% (yellow) isodose line posterior of the retina. The $95 \%$ isodose line includes a $3 \mathrm{~mm}$ margin around the tumour.

\section{Side-effects}

Acute side-effects: patient 8 needed a low dose of corticosteroids $(20-25 \mathrm{mg}$ of prednisolone) to decrease a lid oedema during radiotherapy. One patient suffered from increased pain for a short period of time after radiotherapy. Small areas of local hair loss at the end of SCRT were seen for all patients.

Late side effects: one patient had increased headaches after SCRT. For the patient (case 1) with progressive visual loss, it is hypothesised that treatment may have been given too late, so that a recovery of the visual function was no longer possible. One patient (case 13) developed a radiation retinopathy complicated by a vitreous haemorrhage and cataract 4 years after SCR T. In spite of a vitrectomy, the visual acuity in that eye dropped to light perception. 


\section{DISCUSSION}

The main feature of this study was the improvement of vision after stereotactic radiotherapy. This was sometimes observed during the course of treatment. The majority of visual improvements have been seen early, within 1-3 months after SCRT. This has also been observed by others ${ }^{17,19}$. Our results of visual control are in agreement with other reports, where patients have been treated with fractionated radiotherapy $1,7,8,10,12,13-15,17-19,20,23,24$. We have observed in this multi-centre overview a $95 \%(21 / 22)$ control rate of vision in all cases where a worsening of vision had been documented (16 improved, 5 stabilized). Even if the patient with loss of vision due to toxicity 4 years after treatment is omitted, the control rate is still $90.9 \%$. This conforms to the recent literature ${ }^{1,17,24}$. Until the first patient treated by hypofractionated stereotactic radiosurgery was published ${ }^{14}$, several case-reports showed improvement of vision for all patients treated ${ }^{8,13,15}$. One case treated by intensity modulated radiotherapy is reported with a $100 \%$ restitution of vision ${ }^{10}$. Highly conformal three-dimensional radiotherapy techniques were also reported to result in a high visual control for 13 of 15 eyes ${ }^{19,20}$. Summarizing all 118 published cases treated with irradiation alone (including the present study), the overall visual control rate was $91.5 \%$.

Additionally, we have seen a response to irradiation for patients treated for other symptoms such as pain and proptosis or tumour growth prevention to the contralateral side. A pain reduction was achieved for 6 out of 7 patients, proptosis improved in 1 of 6 , and tumour growth was controlled for all patients. If both treatment results of visual outcome and symptomatic response are pooled together, the overall treatment aim has been reached in this study for 28 out of 35 indications ( 80 $\%)$. No recurrence was observed, which may be due to the relatively short follow-up.

Although the period of follow-up is still too short to draw a definite conclusion, preliminary reports seem to indicate less late toxicity with stereotactic or highly conformal radiotherapy than in studies using conventional radiotherapy ${ }^{26}$. Turbin et. al. published data from a multi-centre study where patients were treated in 4 different way ${ }^{26}$. The patients treated by radiotherapy alone suffered from only half the complication rate $(33 \%)$ compared to those treated by post-operative radiotherapy $(62.5 \%)$. The latter are as high as for those treated by surgery alone $(66.7 \%)$. Complications reported after irradiation alone were retinopathy, vascular occlusion, iritis and temporal lobe atrophy. They may have been caused by the use of larger radiotherapy fields, and different radiotherapy treatment techniques used in multiple centres. Recently published results of highly conformal and stereotactic radiotherapy disclosed a lower complication rate, especially no more case of temporal lobe atrophy. For example, in this study and the study published by Narayan et. al. ${ }^{20}$ using 
3D-CRT, late side-effects were observed in 3 of 14 and 3 of 18 treated eyes (16$20 \%$ ) with useful vision. However, those side effects were seen only after a minimum follow-up period of 38 months. The only patient in this study with serious late side effects (vitreous haemorrhage) was seen after a follow-up period of 48 months, where a stabilization of vision was observed after radiotherapy.

In irradiating tumours of the orbit, the main difficulty is due to the fact that the optic nerve passes through the target itself, and therefore is at risk for late side-effects. Cranial nerves within the target are spared by fractionation. This is even better accomplished with the use of a low fraction size: if the daily fraction size is kept to $1.9 \mathrm{~Gy}$ or below, and a total dose to the tumour of 50.4 to $54 \mathrm{~Gy}$ is used (as in this study). The risk of optic nerve injury is low with $<2 \%$ at 10 years ${ }^{9,22}$. This also refers to the chiasm, the contralateral optic nerve and the retina as well, which are located close to the target volume and which are best spared by the use of stereotactically guided radiotherapy. Most patients in this study received between $10 \%$ and $40 \%$ of the prescribed dose to the chiasm, and the dose to the retina could be reduced for smaller tumours not reaching the eyeball due to the steep dose fall-off with SCRT. The only patient with a retinopathy seen in this study was treated with a fraction size of $2 \mathrm{~Gy}$.

Another organ-at-risk is the pituitary gland. A minimum dose of $50 \mathrm{~Gy}$ to the anterior part of the gland has been described to be at risk for an increased incidence of an endocrinopathy ${ }^{21}$. In this study, the dose could be kept under 50 Gy for the ONSM with a retro-orbital location. The hypothalamus as part of the endocrine hypothalamic-pituitary axes, not taken into considerati-on as OAR in this study for logistic reasons, is reported being more radiosensitive than the pituitary gland itself ${ }^{3}, 16,21$.

In this multi-centre study, the patient fixation, treatment planning and dose prescription were the same and the follow-up scheme was similar with the same imaging and ophthalmologic examinations. We think therefore, that the results of the 4 different institutions are comparable concerning the treatment outcome.

Given this excellent functional and visual outcome observed in this patient group and by others, fractionated stereotactic radiotherapy can be recommended as the treatment of choice. As there is usually no restoration of any vision in blind eyes, SCRT should be offered after the first proof of visual deterioration. A short median follow-up as in this study was sufficient to demonstrate treatment results of improvement in visual function. A definite conclusion concerning the efficacy and toxicity can, therefore, only be drawn after a longer follow-up. 


\section{REFERENCES}

1. Andrews DW, Faroozan R, Yang BP et al. Fractionated stereotactic radiotherapy for the treatment of optic nerve sheath meningiomas: Preliminary observations of 33 optic nerves in 30 patients with historical comparison to observation with or without prior surgery. Nourosurg 2002;51:890-904.

2. Cantore WA: Neural orbital tumors. Curr Opin Ophthalmol. 2000 Oct;11(5):367-71.

3. Constine LS, Woolf PD, Cann D, et al: Hypothalamic-pituitary dysfunction after radiation for brain tumours. N Engl J Med 1993;328:87-94.

4. Cristante L. Surgical treatment of meningiomas of the orbit and optic canal: A retrospective study with particular attention to the visual outcome. Acta Neurochir(Wien) 1994;126:27-32.

5. Dutton JJ. Optic nerve sheath meningiomas. Surv Ophthalmol 1992;37:167-183.

6. Emami B, Lyman J, Brown A ct al: Tolerance of normal tissue to therapeutic radiation. IntJ Radiat Oncol Biol Phys 1991;21:109-122.

7. Fineman MS and Augsburger JJ. A new approach to an old problem. Surv Ophthalmol 1999; 43(6):519-24

8. Goh KY, Yeow YK: Optic nerve sheath meningioma-report of three cases. Ann Acad Mcd Singapore $1994 ; 23(3): 406-11$

9. Goldsmith BJ, Rosenthal SA, Wara WM et al. Optic neuropathy after irradiation of meningioma. Radiology 1992;185:71-76.

10. Grant W III and Cain RB. Intensity modulated conformal therapy for intracranial lesions. Med Dosim 1998; 23(3):237-41

11. ICRU Report 50; Prescribing, recording, and reporting photon beam therapy. International Commission on radiation units and measurements, Bethesda, Maryland 20814 USA. (1993)

12. Ito $M$, Ishizawa $A$, Miyaoka $M$, Sato $K$, Ishii S. Intraorbital meningiomas: Surgical management and role of radiation therapy. Surg Neurol 1988; 103:59-60.

13. Kennerdell JS, Maroon JC, Malton M, Warren FA. The management of optic nerve sheath meningiomas. Am J Ophthalinol 1988;106:450457.

14. Klink DF, Miller NR, Williams J. Preservation of residual vision 2 years after stereotactic radiosurgery for a presumed optic nerve sheath meningioma. J Neuroophthalmol 1998; $18(2): 117-20$

15. Lee AG, Woo SY, Miller NR et al. Improvement in visual function in an eye with a presumed optic nerve sheath meningioma after treatment with three-dimensional conformal radiation therapy. J Neuroophthalmol 1996; 16(4):247-51

16. Littley MD, Shalet SM, Beardwell CG ot al: Hypopituitarism following external radiotherapy for pituitary tumours in adults. QJ Med 1989;70:145-160

17. Liu JK, Forman S, Hershewe GL, Moorthy CR, Benzil DL. Optic nerve sheath meningiomas: visual improvement after stereotactic radiotherapy. Ncurosurg 2002;50:950-57.

18. Montgomery AB, Griffin T, Parker RG, Gerdes AJ. Optic nerve glioma: the role of radiation therapy. Cancer 1977; 40(5):2079-80 
19. Moyer PD, Golnik KC, Breneman J. Treatment of optic nerve sheath meningioma with three-dimensional conformal radiation. Am J Ophthalmol 2000; 129(5):694-6

20. Narayan S, Cornblath WT, Sandler HM, Elner V, Hayman JA. Preliminary visual outcomes after three-dimensional conformal radiation therapy for optic nerve sheath meningioma. Int J Radiat Oncol Biol Phys 2003;56:537-543.

21. Pai HH, Thornton A, Katznelson L et al. Hypothalamic/pituitary function following high-dose conformal radiotherapy to the base of skull: demonstration of a dose-effect relationship using dose-volume histogram analysis. Int $J$ Radiat Oncol Biol Phys 2001;49(4):1079-1092.

22. Parsons JT, Bova FJ, Mendenhall WM et al. Response of the normal eye to high dose radiotherapy. Oncology 1996;10:837-848.

23. Pitz S, Becker G, Schiefer U et al. Stereotactic fractionated irradiation of optic nerve sheath meningioma: a new treatment altemative. $\mathrm{Br} J$ Ophthalmol 2002;86:1265-8.

24. Smith JL, Vuksanovic MM, Yates BM, Bienfang DC. Radiation therapy for primary optic nerve meningioma. J Clin Ncuroophthalmol 1981; 1(2):85-99

25. Tholen AM, Meister A, Bernasconi PP, Messmer EP: Radiotherapy for choroidal neovascularization in age-related macular degeneration. A pilot study using low-versus high-dose photon beam radiation. Ophthalmologe 1998;10:691-8.

26. Turbin RE, Thompson CR, Kennerdell JS, Cockerham KP, Kupersmith MJ. A long-term visual outcome in patients with optic nerve sheath meningioma managed with observation, surgery, radiotherapy, or surgery and radiotherapy. Ophthalmology 2002;109:890-900 


\section{APPENDIX}

\section{SPARING OF THE MACULA}

\section{Dose-volume-histogranis of three cases are shoun in the figure below.}

Dose-volume-histograms for the retina. As the retina itself cannot be visualized on a CT scan an approximate area around the optical nerve bulb was defined. Three cases are presented: Top left and bottom left are examples (cases 1 and 4) with a tumour located in the middle and anterior part of the optical nerve and comes close to the retina. Top right (case 7) is an example of a tumour located in the middle part of the optic nerve leaving more space between tumour and the eye. In all three cases a good sparing of the retina was reached.
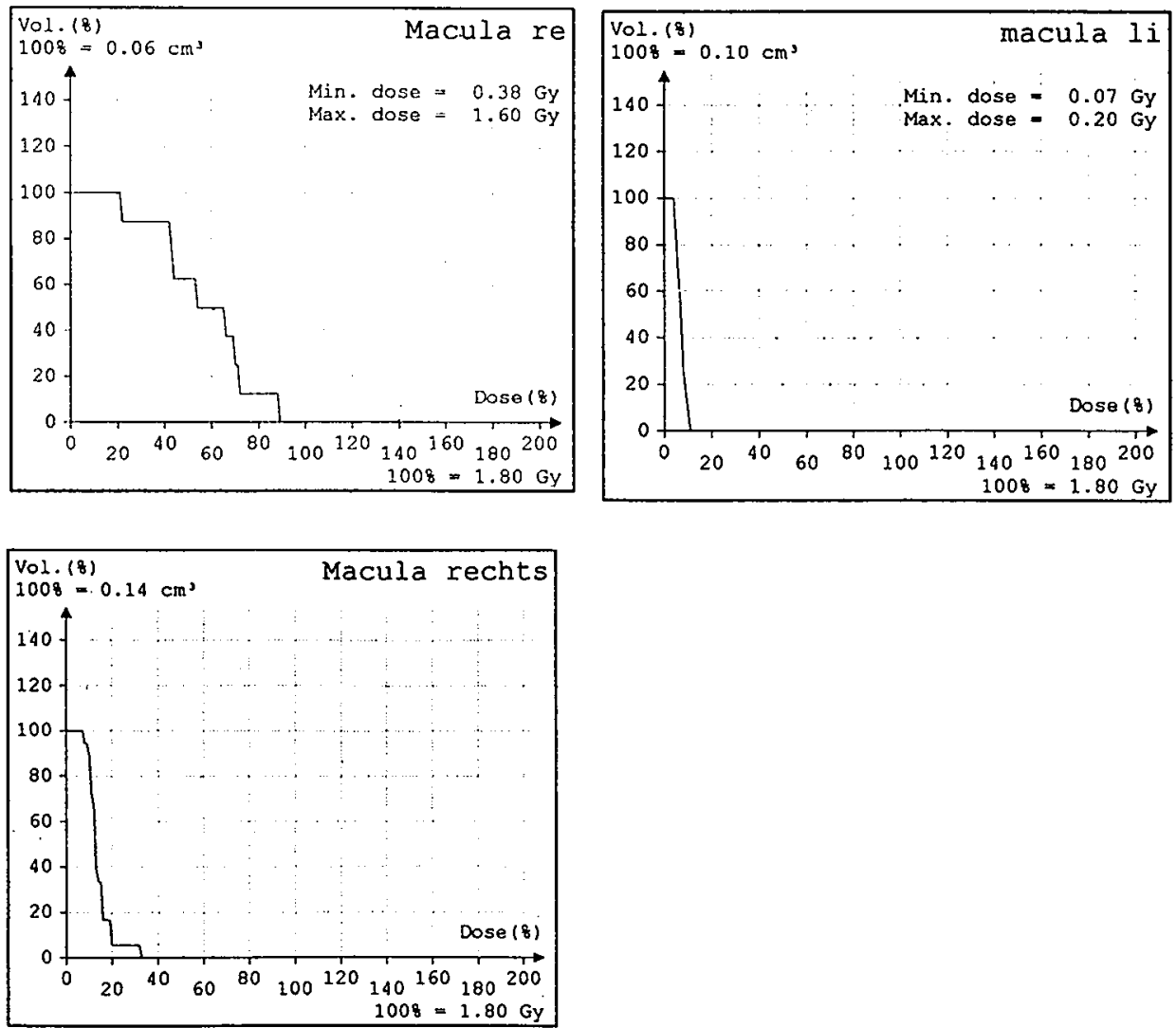
CHAPTER 8

\section{Stereotactically guided conformal radiotherapy for progressive low grade gliomas of childhood}

Frank H. Saran, Brigitta G. Baumert, Vincent S. Khoo, Elizabeth J. Adams, Alan P. Warrington and Michael Brada 


\section{Abstract}

Purpose: To describe the rationale, technique, and early results of stereotactically guided conformal radiotherapy (SCRT) in the treatment of progressive or inoperable low-grade gliomas (LGGs) of childhood.

Methods and Materials: Between September 1994 and May 1999, 14 children (median age 6 years, range 5-16) with LGG were treated with SCRT at the Royal Marsden NHS Trust. Tumors were located at the optic chiasm $(n=9)$, third ventricle $(n=2)$, hypothalamus, craniocervical junction, and pineal region (each $n=1$ ). Four patients received chemotherapy before SCRT. Immobilization was in a Gill-ThomasCosman frame $(n=12)$ and subsequently in a specially designed pediatric version of the frame $(n=2)$. Stereotactic coordinates and the tumor were defined by CT scanning with a fiducial system and MRI fusion. The median tumor volume was $19.5 \mathrm{~cm}^{3}$ (range 7.5-180). The planning target volume was defined as the area of enhancing tumor plus a 5-10 mm margin. The treatment technique consisted of 4 isocentric, noncoplanar, conformal, fixed fields. Treatment was delivered in 30-33 daily fractions to a total dose of 50-55 Gy.

Results: SCRT was well tolerated, with transient hair loss the only acute toxicity. The median follow-up was 33 months (range 2-53). At 6 months after SCRT, 4 of 12 children with neurologic deficits improved and 5 remained stable. Twelve children were available for MRI evaluation. Two had a complete response, 6 a partial response, and 4 stable disease. One child with optic chiasm glioma had local progression at 25 months, and 1 developed diffuse leptomeningeal disease without local progression at 27 months. The 3 -year local progression-free survival and overall survival rate after SCRT was $87 \%$ and $100 \%$, respectively, compared with $89 \%$ and $98 \%$ for an historic control treated with conventional RT. New endocrine deficiencies were noted in 2 children after a follow-up of 20 and 23 months.

Conclusion: SCRT is a feasible, high-precision technique of RT for children with LGGs for whom RT is considered appropriate. The local control and acute toxicity of SCRT are comparable to a historic control of patients with conventionally delivered RT. The frequency of delayed hypothalanuc-pituitary axis dysfunction reflects tumor location adjacent to the hypothalamus and pituitary. Additional follow-up is required to demonstrate that SCRT contributes to a reduction in treatment-related late toxicity, while maintaining the local control achieved with conventionally delivered RT in children with progressive LGGs.

Keyurords: Low-grade gliomas, Pediatric malignancy, Stereotactically guided conformal radiotherapy, Three-dimensional planning. 


\section{INTRODUCTION}

Primary central nervous system tumors are the most common solid tumors of childhood. The most frequent histopathologic tumor entities are gliomas, approximately $80 \%$ of which are low-grade tumors (World Health Organization [WHO] Grades 1 and 2). Low-grade gliomas (LGGs) of childhood are a heterogeneous group with diverse clinical behavior ${ }^{1,2}$, ranging from indolent tumors, such as those associated with neurofibromatosis type 1 and juvenile cerebellar pilocytic astrocytomas, to rapidly progressive and metastasizing intracranial tumors. Complete surgical excision is the treatment of choice in accessible tumors, with 80-95\% long-term local control rates and survival using modern surgical techniques. Patients with LGGs not amenable to macroscopic complete surgical resection without unacceptable morbidity, especially optic chiasmal gliomas, are usually offered initial surveillance with specific treatment at the time of progression.

Local radiotherapy (RT) is an effective treatment modality for inoperable and progressive LGGs of childhood, with long-term tumor control rates of $80-90 \%$ and 10 -year survival rates ranging from $63 \%$ to $89 \%{ }^{4}$. In children $>5$ years with progressive and inoperable disease, RT has been considered the first-line treatment of choic $^{5-7}$. However, legitimate concerns exist about the use of $R T$, especially in very young children with a high probability of long-term survival. Late effects in the form of endocrine dysfunction, neurocognitive deficits, and second malignant neoplasms have been attributed to RT. For this reason, chemotherapy is the first-line treatment for very young children, with the aim of delaying definitive $\mathrm{RT}$; response rates have been in the region of $60 \%$ in previously untreated children ${ }^{3}$.

Stereotactically guided conformal RT (SCRT) is a high-precision technique for the delivery of highly conformal dose distributions using conventional fractionated schemes ${ }^{9,10}$. The use of individually shaped, noncoplanar beam fields allows a high homogenous dose to be delivered to the planning target volume (PTV) while minimizing the dose to normal brain tissue and selectively avoiding organs at risk ${ }^{10}$. The high precision of this technique allows one to reduce the safety margins associated with patient movement and setup errors and enhances this benefit further. The feasibility and safety of such an approach in children ${ }^{11}$ and adults ${ }^{12}$ has been reported. A reduction in the amount of normal brain tissue irradiated to high doses has been shown to reduce long-term neurocognitive sequela $e^{8}$ and should therefore improve the risk/benefit ratio for $\mathrm{RT}$ of immature brain tissue in children with LGGs.

Fractionated SCR T has become a part of routine clinical practice for the treatment of benign adult brain tumors. Children requiring localized RT for LGGs have been 
treated with SCRT at our institution since 1994. We report our experience and preliminary results of SCRT in children with inoperable and progressive LGGs.

\section{METHODS AND MATERIALS}

\section{Patient characteristics}

Between September 1994 and May 1999, 14 children with LGGs were treated with fractionated SCRT at the Royal Marsden Hospital. Twelve patients had parasellar tumors ( 9 optic pathway, 2 third ventricle, and 1 hypothalamus), and 1 tumor was located at the craniocervical junction and 1 in the pineal region. The median age at diagnosis was 6 years (range 7 months to 15 years), the median age at SCRT was 8 years (range $5-16$ ) (Table 1 ).

Surgery preceded RT in 12 patients. Total or subtotal resection was performed in 7 patients and biopsy in 5 patients. The histologic features were reported as pilocytic astrocytoma (WHO Grade $1, n=10$ ), fibrillary astrocytoma (WHO Grade 2, $n=1$ ), and oligoastrocytoma (WHO Grade $2, n=1$ ). In 2 patients with optic pathway glioma, the diagnosis was based on imaging and clinical features alone. The median time from diagnosis to SCRT was 22 months (range 1-94), and the median follow-up after surgery was 13 months (range 1-25) (Table 2). Four patients received chemotherapy before SCRT, and the median time after chemotherapy was 49 months (range 21-94).

Six patients were treated for progressive disease after surgery and 4 children for progressive disease after chemotherapy. Three children had neurologic deficits after biopsy, and 1 received SCRT at the parents' request after subtotal resection.

Before RT, 12 (85\%) of 14 children had a neurologic deficit (Table 3). Hypothalamic-pituitary axis dysfunction before RT was present in 7 (50\%) of 14 children (Table 4).

\section{SCRT technique}

Twelve children were immobilized in a standard Gill-Thomas-Cosman frame. The Gill-Thomas-Cosman localization frame provides a highly reproducible daily relocation with an average accuracy of $\pm 1 \mathrm{~mm}^{14,15}$. Two children were treated using a modified system, with immobilization provided by a specially designed, lightweight frame, with a mean reproducibility of $\pm 1.4 \mathrm{~mm}$ [described in detail by Adams et al. ${ }^{16}$ (Fig. 1)]. Stereotactic coordinates were defined using the Brown-Roberts-Wells 
Table 1. Patient details

\begin{tabular}{|c|c|c|}
\hline Variables & Years & No. of patients \\
\hline \multicolumn{3}{|l|}{ Age } \\
\hline Median & 8 & \\
\hline R:mige & $5-16$ & \\
\hline \multicolumn{3}{|l|}{ Sex } \\
\hline Malk & & 6 \\
\hline Female & & 8 \\
\hline \multicolumn{3}{|l|}{ Timing of SCRT } \\
\hline After surgery for progressive discase & & 6 \\
\hline After chemotherapy for progressive disease & & + \\
\hline After biopsy for neurological deficits & & 3 \\
\hline After primary surgery for residual tumor & & 1 \\
\hline \multicolumn{3}{|l|}{ Location } \\
\hline Optic chinsm & & 9 \\
\hline III. ventricle & $\cdot$ & 2 \\
\hline Hypothalamus & & 1 \\
\hline Craniocervical junction & & 1 \\
\hline Pincal region & & 1 \\
\hline \multicolumn{3}{|l|}{ Histologic features } \\
\hline Pilocytic astrocytomas & & 10 \\
\hline Oligonstrocytoma $\left(1 \mathrm{I}^{\circ}\right)$ & & 1 \\
\hline Fibrillary astrocytoma $\left(11^{\circ}\right)$ & & 1 \\
\hline Unverified & & 2 \\
\hline \multicolumn{3}{|l|}{ Surgery } \\
\hline Subtotal resection & & 7 \\
\hline Biopsy & & 5 \\
\hline Nonc & & 2 \\
\hline \multicolumn{3}{|l|}{ Chenotherapy } \\
\hline Yes & & 4 \\
\hline No & & 10) \\
\hline \multicolumn{3}{|l|}{ Neurofibromatosis } \\
\hline Yes & & 1 \\
\hline No & & 13 \\
\hline
\end{tabular}

Abbreviation: SCRT stereotactically guided conformal radiotherapy.

fiducial system ${ }^{13-15}$. All patients underwent MRI and CT scanning with i.v. contrast. CT scanning in the frame was performed with a 3-mm slice thickness and 3-mm table feed over the tumor region and top of the skull and a 5-mm slice thickness and 6-mm table feed outside the target area.

The MRI data set was automatically registered with the CT scans using ImageFusion (Radionics, Burlington, MA) before contouring of the target and organs at risk. The gross tumor volume (GTV) was defined as the region of contrast enhancement on MRI, and clinical organs at risk were delineated in all relevant slices. GTVs ranged from 6.3 to $180 \mathrm{~cm}^{3}$ (median 15.2). The clinical target volume was defined as the GTV plus 3-8 $\mathrm{mm}$ for well-demarcated pilocytic astrocytomas and a $10-\mathrm{mm}$ margin for poorly defined nonpilocytic astrocytomas. The PTV was the clinical target vol- 
Table 2. Timing of individual treatment cpisodes

\begin{tabular}{|c|c|c|c|c|c|c|c|}
\hline Pr.no & $\begin{array}{l}\text { Interval } \\
\text { between } \\
\text { diagnosis } \\
\text { and first } \\
\text { nonsurgical } \\
\text { treatment } \\
\text { (mo) }\end{array}$ & $\begin{array}{l}\text { First } \\
\text { treatment }\end{array}$ & $\begin{array}{l}\text { Interval } \\
\text { between first } \\
\text { treatment and } \\
\text { second treat- } \\
\text { ment (mo) }\end{array}$ & Second treatment & $\begin{array}{l}\text { Interval } \\
\text { between } \\
\text { second } \\
\text { and third } \\
\text { treatment } \\
\text { (mo) }\end{array}$ & Treatment & $\begin{array}{l}\text { Interval } \\
\text { between } \\
\text { diagnosis } \\
\text { and } \\
\text { SCRT } \\
\text { (mo) }\end{array}$ \\
\hline 1 & 2 & SCRT & & & & & 2 \\
\hline 2 & 1 & Carbo,VC:R & 9 & SCRT & & & 21 \\
\hline 3 & 22 & SCRT & & & & & 22 \\
\hline 4 & 7 & SCRT & & & & & 7 \\
\hline 5 & 8 & SCRT & & & & & 8 \\
\hline 6 & 1 & Carbo.VCR & 12 & Carbo.VC.R & 34 & SCRT & 54 \\
\hline 7 & 24 & SC:RT & & & & & 24 \\
\hline 8 & 1 & Carbo.VCR & 12 & SCRT & & & 27 \\
\hline 9) & 25 & SCRT & & & & & 25 \\
\hline 10) & 1 & $\mathrm{SCIRT}$ & & & & & 1 \\
\hline 11 & 15 & SCRRT & & & & & 15 \\
\hline 12 & 1 & Carbo & 6 & AIDR. CTID, VCR & 60 & SCRT & 94 \\
\hline 13 & 24 & SCRT & & & & & 24 \\
\hline 14 & 6 & SCIRT & & & & & 6 \\
\hline
\end{tabular}

Abbreviations: Pt. no. patient number; Carbo carboplatin; VCR vincristine; ADR adriamycin; ACTD actinomycin D; SCRT stercotactically guided conformal radiotherapy.

Table 3. Clinical and radiological disease status after SCRT

\begin{tabular}{|c|c|c|c|c|c|c|}
\hline No. & $\begin{array}{l}\text { Follow- } \\
\text { up (mo) }\end{array}$ & Pretreatment neurology status & $\begin{array}{l}\text { Neurologic status } \\
6 \text { mo after SCRT }\end{array}$ & $\begin{array}{l}\text { Maximal } \\
\text { imaging } \\
\text { response }\end{array}$ & $\begin{array}{l}\text { Local response at } \\
\text { last follow-up }\end{array}$ & $\begin{array}{l}\text { Time to } \\
\text { progres- } \\
\text { sion (mo) }\end{array}$ \\
\hline 1 & +6 & Blind $R$, reduced VA L & Vision stable & S1) & $\mathrm{Sl})$ & \\
\hline 2 & +1 & 1)ecreased VA $R+L$ & Vision inproved & $P R$ & $P R$ & \\
\hline 3 & 36 & None & None & CR & $\mathrm{CR}$ & \\
\hline+ & 35 & Nonc & None & $P R$ & PR & \\
\hline 5 & 25 & $\begin{array}{l}\text { Decreased VA } R+L \\
\text { Homonymous hemianopia } R\end{array}$ & Vision improved & PR & Progression & 25 \\
\hline 6 & 17 & Bilat. VI. nerve palsy & Stable & SI) & $\mathrm{Si})$ & \\
\hline 7 & 38 & Blind $R$, reduced visual ficld $L$ & Vision stable & S1) & $\mathrm{SI}$ & \\
\hline 8 & 53 & Hemiparesis R & None & $C R$ & $\mathrm{CR}$ & \\
\hline 9 & 28 & $\begin{array}{l}\text { Decreased VA } R+L \\
\text { temp. field loss } R\end{array}$ & vision stable & P'R & $\begin{array}{l}\text { PR, dissemination } \\
\text { outside RT-ficld }\end{array}$ & 27 \\
\hline 10 & 18 & Hemiparesis L & $\begin{array}{l}\text { Hemiparesis } \\
\text { improved }\end{array}$ & $P R$ & $\mathrm{PR}$ & \\
\hline 11 & 23 & $\begin{array}{l}\text { Decreased VA } R+L \\
\text { Homonyme hemianopia } L\end{array}$ & Vision improved & $\mathrm{PR}$ & $\mathrm{PR}$ & \\
\hline 12 & 45 & Decreased VA $R+L$ & Vision stable & SI) & SI) & \\
\hline 13 & 2 & $\begin{array}{l}\text { Incontinence, medius rectus } \\
\text { palsy R }\end{array}$ & Vision stable & SI) & NA & \\
\hline $1+$ & 0 & Epilepsy. ataxia & NA & $\mathrm{NA}$ & $\mathrm{NA}$ & \\
\hline
\end{tabular}

Abbreviations: $\mathrm{R}=$ right eye; $\mathrm{L}=$ left eyc; $\mathrm{VA}=$ visual acuity; $\mathrm{CR}=$ complete response; $\mathrm{PR}=$ partial responsc; $\mathrm{SD}=$ stable disease, $\mathrm{NA}=$ not analysed; other abbreviations as in Table 2. 
Table 4. Endocrine function before and after SCRT

\begin{tabular}{|c|c|c|c|}
\hline Pt. no. & $\begin{array}{l}\text { Follow- } \\
\text { up (mo) }\end{array}$ & $\begin{array}{l}\text { Hypothalamic-pituitary axis function before } \\
\text { treatment }\end{array}$ & $\begin{array}{l}\text { Hypothalamic-pituitary axis function } \\
\text { after SCRT }\end{array}$ \\
\hline 1 & 39 & None & None \\
\hline 2 & $3+$ & Git deficit, precocions puberty & $\begin{array}{l}\text { (iH deficit. precocious pulerty treatment } \\
\text { (leuporelin) }\end{array}$ \\
\hline 3 & 29 & $\begin{array}{l}\text { l'anhypopituitarism, (GH, I)I)AVP, cortisonc, } \\
\text { thyroxinc), liyperplagia }\end{array}$ & $\begin{array}{l}\text { Panhypopituitarism, (GH, DI)AVP, cortisone. } \\
\text { thyroxinc), hyperplagia }\end{array}$ \\
\hline+ & 28 & $\begin{array}{l}\text { (iH deficit. diabetes insipidus, hypotbalamic } \\
\text { obesity }\end{array}$ & $\begin{array}{l}\text { CiH deficit, diabetes iusipidus, hypothalamic } \\
\text { obesity, hypothyroidism ( } 23 \text { mo after SCIRT) }\end{array}$ \\
\hline 5 & 18 & None & None \\
\hline 6 & 10 & (it- deficit, precocious puberty & $\begin{array}{l}\text { GH deficit, precocious puberty treatment } \\
\text { (leuporclin) }\end{array}$ \\
\hline 7 & 31 & None & l'recocious puberty (20) mo after SC.RT) \\
\hline 8 & 46 & None & None \\
\hline 9 & 21 & (iH deficit, precocious puberty & $\begin{array}{l}\text { (iH deficit, precocions puberty treatment } \\
\text { (leuporelin) }\end{array}$ \\
\hline 10 & 11 & None & None \\
\hline 11 & 16 & (il- deficit, hypothalamic obesity & (iH deficit hypothalamic obesity \\
\hline 12 & 38 & $\begin{array}{l}\text { CiH deficit, anterior pituitary dysfunction, } \\
\text { epilepsy }\end{array}$ & Cit deficit, ant. pituitary dysfunction, epilepsy \\
\hline 1.3 & 2 & Nonc & Nome \\
\hline 14 & 2 & None & Nonc \\
\hline
\end{tabular}

Abbreviations: $\mathrm{GH}=$ growth hormonc; $\mathrm{DDAVP}=$ desmopressin acctate; other abbreviations as in Table 2.

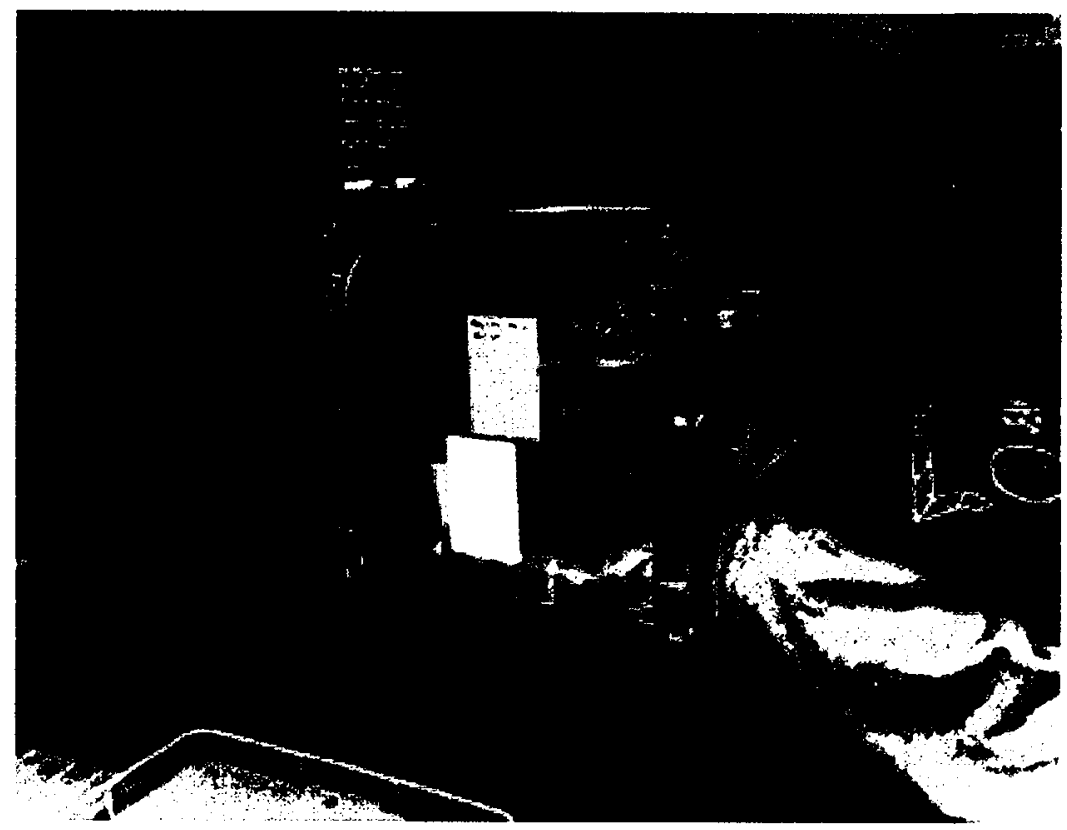

Fig. 1. Treatment setup with relocatable children's frame. 
ume plus $2 \mathrm{~mm}$. Planning was carried out using CADPLAN (Varian Medical Systems, Palo Alto, CA) or GE Target $1 / 2$ (General Electric Medical Systems, Milwaukee, WI $)^{17}$. A single isocenter was used with 4 noncoplanar fixed fields consisting of 2 lateral posterior inferior oblique beams and 2 sagittal beams ${ }^{10}$ maximally separated. Each beam was conformed to the projection of the PTV either by a conformal lead alloy block $(n=10)$ or a multileaf collimator $(1-\mathrm{cm}$ leaf width at isocenter) $(n=4)$ using three-dimensional reconstruction and beam's eye view facilities ${ }^{18}$.

\section{Dose and fractionation}

All patients received fractionated SCR T without general anesthesia on a daily outpatient basis. Doses were normalized using the International Commission on Radiation Units and Measurement (ICRU)-50 criteria and prescribed to the isocenter. The total dose delivered was $50 \mathrm{~Gy}$ in $33(n=9)$ or $30(n=4)$ fractions and $55 \mathrm{~Gy}$ in 33 fractions for 1 patient with a fibrillary astrocytoma of the pineal region.

\section{Quality assurance}

The assessment of setup accuracy was performed by the use of either weekly port films or daily portal imaging of all verifiable treatment fields (Theraview Electronic Portal Imaging Device, InfiMed, Liverpool, NY). These were compared with digital reconstructed radiographs and/or simulation films. All treatments were delivered with a setup error of $<2-3 \mathrm{~mm}$. The quality assurance procedure has been previously described in detail ${ }^{10,19}$.

\section{Follou-up}

Patients were followed in a joint neuro-oncology and endocrine clinic with regular neurologic examinations and endocrine assessments. Patients with visual deficits or optic chiasm gliomas had regular ophthalmologic assessments by a pediatric ophthalmologist on a 3-6-month basis. A baseline MRI scan was obtained 4-6 weeks after completion of SCRT. Additional MRI scanning was performed on a 6-month basis for the first 2 years and annually thereafter. Follow-up was every 3 months for the first 2 years, 6 months for the next 3 years, and annually thereafter. All pretreatment and follow-up images were reviewed again by an independent neuroradiologist. The radiologic response was assessed by MRI and described at the time of maximal response (Table 3 ). The clinical response was assessed at each appointment by a clinical and neurologic examination. The reported response was that noted at the last follow-up evaluation or at the time of maximal response. The median follow-up from SCRT was 33 months (range 2-53). 


\section{Historical cohort}

Between 1955 and 1993, 33 children with optic pathway glioma were treated with conventional external beam $\mathrm{RT}$ using a coplanar nonconformal 3-field technique to a dose of 50 Gy in 30-33 fractions. Patient, disease, and treatment characteristics are shown in Table 5 . The details of treatment have been previously reported ${ }^{20}$.

\section{RESULTS}

Fourteen children with LGGs were treated with SCRT. The neurologic deficit improved after SCRT in 5 patients. Eight of 14 children presented with a visual deficit. At the last follow-up visit, vision had improved in 2 , stabilized in 4 , and worsened in 2 . The neurologic deficit remained stable in 5 patients. Both children without symptoms before treatment remained asymptomatic. On neuroimaging studies, 2 children had complete disappearance of the tumor (complete response), 6 had a reduction in tumor size (partial response), and 4 had stable disease. One child (Patient 5) with an optic chiasm glioma had local relapse 25 months after SCRT after a previously documented partial remission. One child (Patient 9) with a pilocytic astrocytoma of the optic chiasm developed diffuse leptomeningeal dissemination outside the irradiated volume 27 months after treatment (Table 3). At 3 years, the local progression-free survival and overall survival rate was $87 \%$ and $100 \%$, respectively (Figs. 2 and 3 ).

Patients treated with stereotactic RT were compared with a historical cohort of 33 children with optic glioma who were treated from 1955 to 1993 with conventional RT techniques. The median follow-up of the historical cohort was 11 years, with an overall survival and progression-free survival rate of $98 \%$ and $89 \%$ at 3 years and $96 \%$ and $84 \%$ at 10 years, respectively. A comparison of the SCRT and historical cohorts did not demonstrate a statistically significant difference in local tumor control and survival ( $p=0.5$ and 0.6; Figs. 2 and 3 ).

SCRT was well tolerated. Transient hair loss at the entrance of the treatment beams occurred in all children. No other acute toxicity was observed. Neurocognitive evaluations were not prospectively performed. Late neurocognitive sequelae were suspected in 2 patients: Patient 12 developed learning difficulties 2 years after SCRT and Patient 13 was reported to have a reduction of short-term memory function 6 months after SCRT completion. Two patients developed a new hypothalamic-pituitary axis dysfunction at 20 and 23 months after SCR T (Table 4). All other hypothalamic-pituitary axis disorders were present before SCRT (Table 4). 


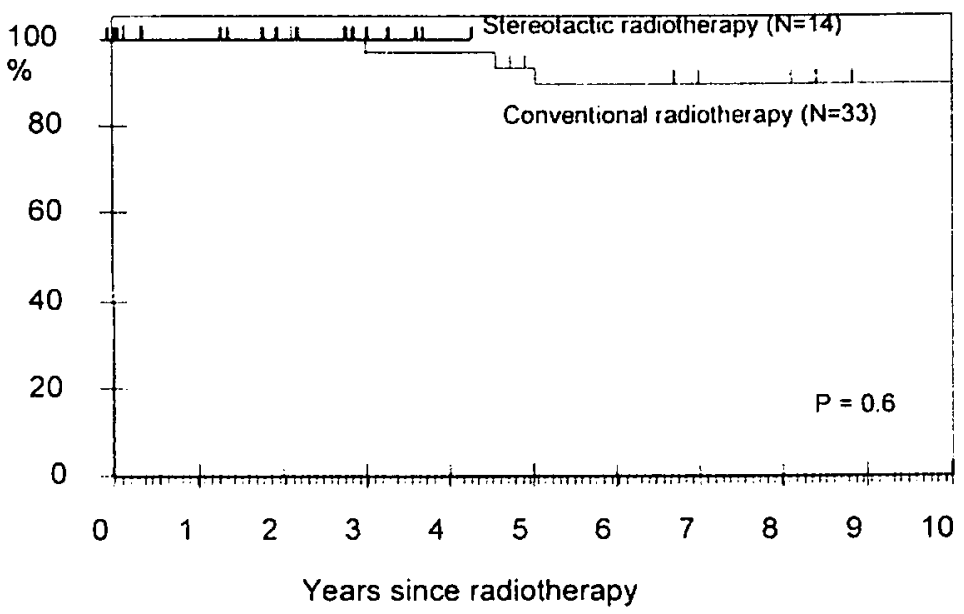

Fig. 2. Comparison of children treated with conventional RT techniques and SCRT: Overall survival from the first day of RT.

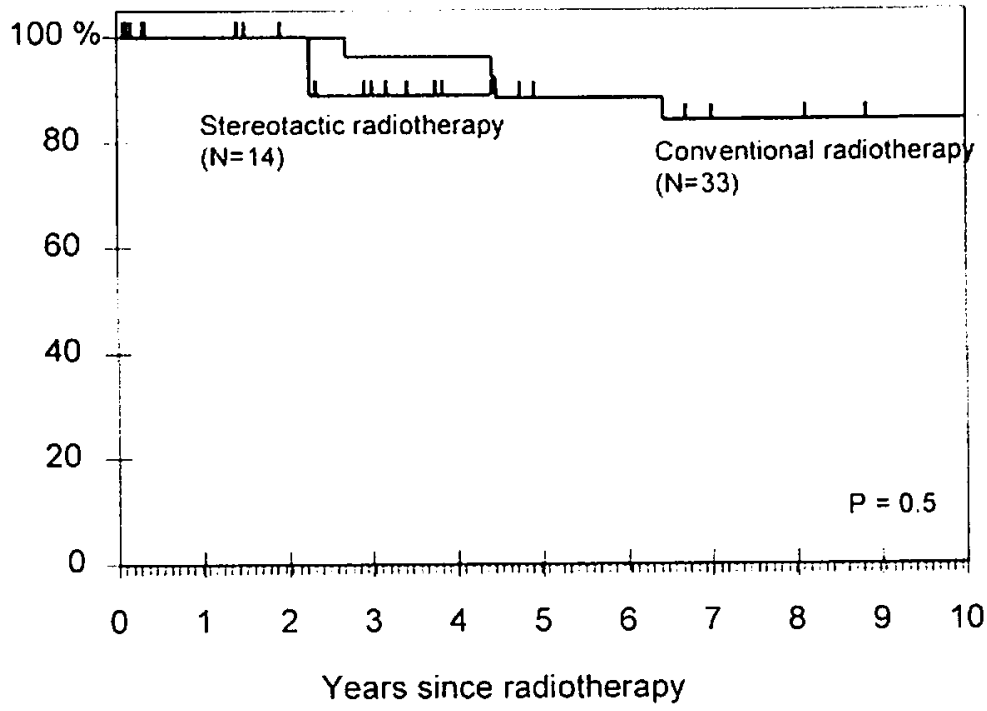

Fig. 3. Comparison of children treated with conventional RT techniques and SCRT: Progression-free survival from the first day of RT. 
Table 5. Historical cohort of 33 children with optic gliomas.

Variables

Patients (n)

RMH presentation (y)

Radiotherapy (y)

Age at RMH presentation (y)

Median

R.unge

Age at radiotherapy $(y)$

Median

Range

Gender

Male

Fenale

Tumor site

Optic nerve only

Chiasm only

Chiasm + other contiguous structure

Optic nerve + chiasm

Optic nerve + chinsm + other contiguous structure

Optic nerve + chiasm + Posterior optic tract

Nerve + chiasm + Posterior optic tract + other contiguous structures

Preliminary treatment intent

Observation alone

Radiotherapy

Indication for treatment

Residual disease

Progressive disease

Neurologic impairment

Surgery

None

Shunt

Craniocomy (no biopsy)

Biopsy

Partial removal

Macroscopic removal

Chemotherapy

Neurofibromatosis

Follow up

Median (y)

Range
Years

No. of patients

1950-1991

1951-1991

3

(0)-15

5

(1)-24

16

17

3

5

1

16

5

2

1

2 mo to $44 y$

Abbreviation: RMH Royal Marsden Hospital.

\section{DISCUSSION}

Low-grade pilocytic astrocytomas (WHO Grade 1) in children are predominantly located in the cerebellum and suprasellar region commonly involving the optic pathway, with occasional hypothalamic or third ventricular region tumors ${ }^{20}$. Optic pathway or chiasm gliomas are frequently associated with neurofibromatosis type I and can have a clinically indolent behavior. Diffuse low-grade astrocytomas (WHO 
Grade 2), for example, fibrillary astrocytomas and oligoastrocytomas, are uncommon and can occur at any site in the brain ${ }^{21,22}$. Both Grade 1 and 2 childhood gliomas have an excellent long-term prognosis ${ }^{22,23}$ and have therefore been analyzed together.

The treatment of LGGs is site dependent. The treatment of choice for patients with accessible tumors is complete excision, particularly in the posterior fossa. $\mathrm{RT}$ is generally reserved for older children with symptomatic, progressive, and unresectable LGGs or those in whom chemotherapy is failing. Chemotherapy is currently used as primary therapy in young children ( $<4-6$ years old) with progressive tumors.

The 100\% 3-year survival and 87\% 3-year progression-free survival rates after SCRT are in keeping with other published studies of optic gliomas treated with fractionated stereotactic RT. Debus et al. ${ }^{11}$ reported a $10 \%$ in-field recurrence rate at a median follow-up of 3 years $(7$ children and 3 adults). Twenty-eight children with unresectable low-grade astrocytomas were treated with fractionated stereotactic RT at the Massachusetts General Hospital ${ }^{5}$. Local control was $100 \%$ at 2 years, with 1 child developing disseminated disease outside the treated volume.

The outcome after SCRT was compared with the results of a cohort of children with optic pathway gliomas treated with conventional RT at the Royal Marsden Hospital. The 3- and 10-year overall survival and progression-free survival rates were $98 \%$ and $89 \%$ and $96 \%$ and $84 \%$, respectively, at a median follow-up of 14 years (Figs. 2 and 3). The results of SCRT were comparable in terms of local tumor control and survival. The overall survival and progression-free survival rates after conventional RT reported by other centers ranged from $80 \%$ to $90 \%$ and $68 \%$ to $89 \%$, respectively, at 10 years $4,6,7,19,24-26$, within the range reported in the present SCRT cohort and the Royal Marsden NHS Trust historical cohort.

Some LGGs diminished in size after irradiation, with a complete remission in $14 \%$, partial remission in $50 \%$, and stable disease in $28 \%$ as determined by MRI after SCRT compared with a baseline scan in our cohort. Similar imaging response rates have been described after conventional $\mathrm{RT}^{6,7,27}$. Tumor responses of $30-50 \%$ are common radiologic findings, and complete remission is the exception.

The precise technical details of SCRT, as well as the required safety margins, have not been agreed on internationally. The current European recommendation (International Society of Pediatric Oncology LGG study protocol) for conventional RT is a safety margin of $0.5-1.0 \mathrm{~cm}$ around pilocytic and $1.0-2.0 \mathrm{~cm}$ around the GTV of other LGGs. The precision of SCRT may allow an additional reduction in the GTV-PTV margins, with a consequent reduction in the volume of normal tissue 
irradiated to high doses. Our data support the assumption that a reduction of clinical target volumes, as enabled by the use of SCRT, does not compromise local control compared with conventionally delivered $\mathrm{RT}$ in patients with progressive or symptomatic, inoperable LGGs. However, $64 \%$ of patients with this type of LGG have been reported to have infiltrated the surrounding parenchyma histopathologically despite a well-demarcated appearance on $\mathrm{MRI}^{28}$. Therefore, considerable caution is required when setting small margins. The GTV-PTV margins used by other centers for SCR T were $7 \mathrm{~mm}^{11}$ and for radiosurgery $2-3 \mathrm{~mm}^{29,30}$. The margin of $5 \mathrm{~mm}$ (initially $10 \mathrm{~mm}$ ) was chosen to allow for the combined uncertainties of the technique, particularly accounting for the difficulty of GTV definition. Although there is no apparent increase in marginal failures with reduced GTV-PTV margins, the long natural history preclude complete confidence that no risk of late relapse exists from marginal miss, and such a risk should not be underestimated. Any additional reduction in margins should preferably be performed under the auspices of an international prospective study.

SCRT was well tolerated, and no Grade 3 and 4 toxicities occurred. Some centers advocate stereotactic radiosurgery (SRS) as a suitable radiotherapeutic option in children with gliomas. However, 1 treatment-related death after SRS of a malignant brain tumor and several cases of brain necrosis ${ }^{29-31}$ have been reported. For small LGGs $(16-25 \mathrm{~mm})$, no radiosurgery-associated morbidity has been observed after a median follow-up of 19 months $^{32}$. In a series of 25 children treated with SRS alone or in combination with fractionated RT, 4 patients developed transient acute toxicity ${ }^{33}$. Very few patients would be suitable for SRS, because tumors are generally $>2.5$ $\mathrm{cm}$ in the maximal diameter, and most are closely involved with the optic nerve. Fractionated SCRT is the radiotherapeutic treatment of choice in this patient group.

Damage to the hypothalamic-pituitary axis is one of the late sequelae of RT to the central nervous system and causes permanent endocrine dysfunction. One half of our patients treated had a preexisting endocrine deficit or had precocious puberty. After SCRT, 2 children developed a new or additional endocrine deficit during follow-up. One of these had undergone previous surgery involving the hypothalamic area. Childhood tumors amenable to fractionated SCRT often lie in close proximity to the hypothalamus and pituitary gland. Regardless of the method of radiation delivery, a reduction in the frequency of endocrine dysfunction will be difficult to achieve, because it is nearly impossible to avoid irradiating these structures, despite the latest advances in imaging and radiation techniques.

Neurocognitive late sequelae are predominantly seen in young children $\leq 4-5$ years old receiving high-dose RT. In this patient group, observation and chemotherapy 
have been successfully used to delay RT, so no child treated with irradiation in our cohort was $<5$ years old.

SCRT has technical advantages over conventional 3-field coplanar treatment techniques in terms of sparing normal brain tissue to high radiation doses ${ }^{10}$. Perks and colleagues ${ }^{10}$ demonstrated that the use of a 4-field noncoplanar conformal technique compared with a 3-field coplanar conformal technique results in a mean sparing of $22.3 \%$ of extra volume of normal brain at $\geq 80 \%$ isodose and $47.6 \%$ at the $\geq 60 \%$ isodose level. Given that our historic control underwent conventionally planning, a retrospective comparison of GTVs or PTVs between the two techniques was not feasible. However, it is highly likely that at least a similar quantitative benefit of normal brain tissue sparing was achieved in children treated with SCRT compared with the 3-field coplanar, nonconformal historical control group, because the clinical situation was comparable to the patient cohort used by Perks et al. ${ }^{10}$.

The aim of SCRT in children is to reduce the risk of long-term sequelae associated with high-dose RT compared with conventional fractionated irradiation while maintaining the same high local control rates ${ }^{34}$. We did not perform formal prospective neurocognitive evaluations to confirm the hypothetical benefit of SCRT over conventional delivered irradiation in terms of neurocognitive status. However, all patients were interviewed at each visit about their educational progress. Two children reported a change in their cognitive abilities after SCRT that may have be due to the combined effects of all the treatment modalities. One child who had received chemotherapy at 8 months of age and had residual disease for several years before SCRT described new learning difficulties 2 years after $R T$ completion. In a conventionally treated cohort of patients with optic glioma, 20 of 28 patients required special education and 11 of them had a history of pretreatment learning difficulties ${ }^{6}$. Severe intellectual disabilities were also noted in 18 of 33 children treated with RT for optic gliomas at a follow-up of 7 years ${ }^{26}$. Several studies on the cognitive effects in adult patients with pituitary tumors treated with surgery alone or surgery plus RT related the cognitive disorders to surgery, as well as $\mathrm{RT}^{35-37}$. Also, RT clearly contributes to neurocognitive late sequelae in this patient group, and given the variety of clinical courses and direct tumor-related morbidity, it most likely remains a multifactorial process requiring careful individual patient history evaluation.

\section{CONCLUSION}

SCRT is a feasible, high-precision technique of irradiation for children with LGGs that achieves a reduction in the volume of normal brain irradiated to high radiation doses compared with conventional RT. The incidences of acute toxicity of, and local 
control after, SCRT were comparable with the results of conventionally delivered RT. The reported high frequency of hypothalamic-pituitary axis dysfunction reflects the tumor location in close proximity to the hypothalamus and pituitary gland and is not solely a late sequelae related to RT. Longer follow-up of a larger cohort with prospective evaluation is necessary to demonstrate the presumed reduction in late treatment-related toxicity and maintained local control of SCRT compared with conventionally delivered irradiation.

\section{REFERENCES}

1. Dutton J. Gliomas of the anterior visual pathways. Surn Ophthalmol 1994;38:427-452.

2. Liu GT, Lessell S. Spontaneous visual improvement in chiasmal gliomas. Am J Ophthalmol 1992;114:193-201.

3. Packer RJ, Lange B, Ater J, ct al. Carboplatin and vincristine for recurrent and newly diagnosed low grade gliomas of childhood. J Clin Oncol 1993;11:850 -856.

4. Freeman CR, FarmerJP, Montes J. Low-grade astrocytomas in children: Evolving management strategies. Int J Radiat Oncol Biol Plys 1998;41:979 -987.

5. Tarbell $\mathrm{NJ}$, Loeffler JS. Recent trends in the radiotherapy of pediatric gliomas. $J$ Neurooncol 1996;28:233-244.

6. Tao ML, Barnes PD, Billett AL, et al. Childhood optic chiasm gliomas: Radiographic response following radiotherapy and long-term clinical outcome. Int Radiat Oncol Biol Phys 1997;39:579-587.

7. Fisher B, Baumann G, Leighton C. Low-grade gliomas in children; tumour volume response to radiation. J Neurosurg 1998;88:969-974.

8. Gregor A, Cull A, Traynor E, ct al. Neuropsychometric evaluation of long-term survivors of adult brain tumours: Relationship with tumour and treatment parameters. Radiother Oncol 1996;41:55-59.

9. Laing RW, Bentley RE, Nahum AE, $c$ al. Stereotactic radiotherapy of irregular targets: A comparison between static conformal beams and non-coplanar arcs. Radiother Oncol 1993;28:241-246.

10. Perks JR, Jalali R, Cosgrove VP, et al. Optimization of stereotactically-guided conformal treatment planning of sellar and parasellar tumors, based on normal brain dose volume histograms. Int J Radiat Oncol Biol Phys 1999;45: 507-513.

11. Debus J, Kocagoncu KO, Hoss A, ct al. Fractionated stereotactic radiotherapy (FSRT) for optic glioma. Int J Radiat Oncol Biol Phys 1999;44:243-248.

12. Alheit $\mathrm{H}$, Saran $\mathrm{FH}$, Warrington $\mathrm{AP}$, $c$ al . Stereotactically guided conformal radiotherapy for meningiomas. Radiother Oncol 1999;50:145-150.

13. Gill SS, Thomas DG, Warrington AP, ct al. Relocatable frame for stereotactic external beam radiotherapy. Int J Radiat Oncol Biol Phys 1991;20:599 -603. 
14. Graham J, Warrington A, Gill S, et al. A non invasive, relocatable stereotactic frame for fractionated radiotherapy and multiple imaging. Radiother Oncol 1991;21:60-62.

15. Kooy HM, Dunbar SF, Tarbell NJ, et al. Adaptation and verification of the relocatable Gill Thomas Cosman frame in stereotactic radiotherapy. Int J Radiat Oncol Biol Phys 1994; 30:685-691.

16. Adams EJ, Suter BL, Warrington AP, ot al. Design and implementation of a system for treating paediatric patients with stereotactically-guided conformal radiotherapy. Radiother Oncol 2001;60:289 -297.

17. Khoo VS, Adams EJ, Saran F, et al. A comparison of clinical target volumes determined by $\mathrm{CT}$ and MRI for the radiotherapy planning of base of skull meningiomas. Int J Radiat Oncol Biol Phys 2000;46:1309 -1317.

18. Adams EJ, Cosgrove VP, Shepherd SF, et al. Comparison of a multi-leaf collimator with conformal blocks for the delivery of stereotactically guided conformal radiotherapy. Radiother Oncol 1999;51:205-209.

19. Warrington AP, Laing RW, Brada M. Quality assurance in fractionated stereotactic radiotherapy. Radiother Oncol 1994; 30:239 -246.

20. Horwich A, Bloom HJ. Optic gliomas: radiation therapy and prognosis. Int J Radiat Oncol Biol Plys 1985;11:1067-1079.

21. Giannini C, Scheithauer BW. Classification and grading of low-grade astrocytic tumors in children. Brain Pathol 1997; 7:785-798.

22. Gajjar A, Sanford RA, Heideman R, et al. Low-grade astrocytoma: A decade of experience at St. Jude Children's Research Hospital. J Clin Oncol 1997;15:2792-2799.

23. Pollack I. Low-grade gliomas of the cerebral hemispheres in children: An analysis of 71 cases. J Neurosurg 1995;82:536-547.

24. Wong JY, Uhl V, Wara WM, et al. Optic gliomas: A reanalysis of the University of California, San Francisco experience. Cancer 1987;60:1847-1855.

25. Pierce SM, Barnes PD, Loeffler JS, et al. Definitive radiation therapy in the management of symptomatic patients with optic glioma: Survival and long term effects. Cancer 1990; 65:45-52.

26. Cappelli C, Raquin M, Pierre-Kan A. Long term follow-up of 69 patients treated for optic pathway tumours before the chemotherapy era. Arch Dis Child 1998;79:334 -338.

27. Bakardjiev A, Barnes $P$, Goumnerova L. Magnetic resonance imaging after stereotactic radiation therapy for childhood low grade astrocytoma. Cancer 1996;78:864 -873.

28. Coakley K, Hustn J, Schiethauer B. Pilocytic astrocytomas: Well demarcated magnetic resonance appearance despite frequent infiltration histologically. Mayo Clin Proc 1995;70: 747-751.

29. Hodgson D, Goumnerova L, Loeffler JS. Stereotactic radiosurgery (SRS) in the management of pediatric brain tumours. Med Pediatr Oncol 1999;33:163.

30. Weprin BE, Hall WA, Cho KH, et al. Stereotactic radiosurgery in pediatric patients. Pediatr Neurol 1996;15:193-199.

31. Baumann G, Wara M, Larson D. Gamma knife radiosurgery in children. Pediatr Neurostrg 1996;24:193-201. 
32. Somaza SC, Kondziolka D, Lunsford LD, ct al. Early outcomes after stereotactic radiosurgery for growing pilocytic astrocytomas in children. Pediatr Neurosurg 1996;25:109-115.

33. Grabb PA, Lunsford LD, Albright AL, ct al. Stereotactic radiosurgery for glial neoplasms of childhood. Neurosurgery 1996;38:696-701.

34. Smitt MC, McPeak EM, Donaldson SS. The advantages of three-dimensional conformal radiotherapy for treatment of childhood cancer. Radiat Res 1998;150:S170 -S177.

35. Peace KA, Ome SM, Thompson AR, ct al. Cognitive dysfunction in patients treated for pituitary tumours. J Clin Exp Neuropsychol 1997;19:1-6.

36. Guinan EM, Lowy C, Stanhope N, $c t$ al. Cognitive effects of pituitary tumours and their treatments: Two case studies and an investigation of 90 patients. J Neurol Neurosurg Psychiatry 1998;65:870 -876.

37. Grattan Smith PJ, Morris JG, Shores EA, et al. Neuropsychological abnormalities in patients with pituitary tumours. Acta Neurol Sennd 1992;86:626-631. 
CHAPTER 9

General discussion and summary 


\section{INTRODUCTION}

The last 2 decades have witnessed a rapid and immense development of computer technology and its introduction in radiation therapy. This has been instrumental in the adoption of $3 \mathrm{D}$ conformal techniques in the treatment of malignancies. Tools such as computer controlled linear accelerators and treatment delivery, 3D treatment planning, multi-leaf collimators, 3D multi-modality imaging and electronic portal imaging have largely contributed to the technical advancement of radiotherapy. Their combined use resulted in 3D conformal radiotherapy. This can be defined as the tailoring of radiation dose to the treatment volume in three dimensions. More recently, the addition of modulation to the beam's intensity has resulted in intensity-modulated radiotherapy. This technique allows a modulation of dose within the target volume and a better avoidance of surrounding normal tissues than is possible with conventional shielding methods. It is thought that this technique will increase tumour cure rate and decrease long-term morbidity through its potential ability to deliver a dose tailored to the tumour cell density. This is important in the treatment of central nervous system tumours where irradiation is still a mainstay in the treatment concept. It has been demonstrated that the use of CT-based full 3D treatment planning techniques compared with simple 3D planning techniques results in a $30 \%$ reduction in the volume of brain tissue treated to a high-dose level ( $>95 \%$ isodose line $)^{48}$. As a consequence, there is less intellectual impairment in long-term survi vors $^{15}$.

Conformal radiotherapy consists of many steps, which are inter-related. Treatment preparation and delivery of 3D-CRT could be compared with the construction of a chain where the weakest link limits the strength of the chain ${ }^{51}$. The links of the chain are described in Chapter 1.

This thesis follows this chain of events in 3D conformal radiotherapy. Chapter 2 reports on the accuracy of devices used for fractionated stereotactic radiotherapy. The evaluation of different planning methods and radiation techniques are investigated and discussed in Chapters 3 to 5 . Lastly, but of utmost importance, the clinical outcome of SCRT for different tumour entities is investigated in Chapters 6 to 8 .

The objectives of this thesis are detailed at the end of Chapter 1, Section 1.9. In short these are the assessment of pre-requisites of SCRT (e.g. accuracy of the system), to investigate advantages and disadvantages of different beams for 3D-CRT with respect to conformality, sparing of organs-at-risk, and clinical results for different brain lesions. 


\section{ACCURACY}

High precision is the pre-requisite for SCRT as treatment delivery and sparing of normal tissue is a function of the accuracy achieved. Consequently, the accuracy of the stereotactic immobilisation system used in the clinic was investigated (Chapter 2). A fixation system based on a stereotactic mask combined with a mould based on an impression of the upper dentition (= bite-block) or with a simple upper-jaw support for the upper jaw was evaluated. All three forms of fixation (mask alone, mask with bite-block, mask with upper jaw support) were measured by serial check CT scans. The accuracy of patient set-up was studied by assessing the isocentre positions in relation to the patient's anatomical structure with the planning CT as the reference scan. One hundred and fourteen check CT scans of 57 patients were evaluated. There was a significant improvement of the overall displacement and in the cranio-caudal deviation in using the bite block instead of the upper jaw support. The addition of an upper jaw support significantly reduced lateral head rotations compared to the mask system alone. For the largest reduction of non-target tissue irradiation, the use of a custom made bite-block is necessary.

\section{EVALUATION OF CONFORMALITY AND DIFFERENT 3D CRT TECHNIQUES}

The hypothesis that optimisation of stereotactic treatment techniques, either through the use of inverse planned intensity modulated photon beams or through the use of proton beams, has the potential to improve dose conformality when compared to forward planned standard SCRT, was investigated in three comparative planning studies (Chapters 3 to 5 ). The possible gain indicated by these studies could be an improvement in the therapeutic ratio by reducing the dose to normal tissues and as such the risk of morbidity. For these studies patients planned and treated for routine SCRT were selected and replanned for comparison purposes. Tumours located mainly in the base of the skull were chosen for these studies as these are surrounded by several critical structures and are often complex in shape. For completeness multifocal and ellipsoid lesions were used in these studies. For SCRT, conformal avoidance was achieved by using 4 to 6 non-coplanar fixed beams. For intensity modulated treatment plans, inverse planning techniques used were based on specially designed optimisation algorithms to spare critical organs by defining dose constraints and weighting factors. For the proton plans, the spot-scanning technique and energy modulation, deliverable from an isocentrically-mounted gantry were used. 
The first study investigated the use of intensity modulation with SCRT (Chapter 3 ). Ten patients ( 7 with a skull base meningioma) were selected. This resulted in a consistently higher conformality for IMSRT with the largest improvement seen for the complex irregular and multifocal tumour shapes. Sparing of critical structures, especially organs-at-risk within a concavity was also better with IMSRT. However, the volume of normal tissue receiving a low dose (= the $30 \%$ isodose) was larger for IMSRT in 6 out of 10 cases.

The evaluation of SCRT and proton plans for 7 lesions grouped by shape (concave, ellipsoid isolated, superficial and close to an organ at risk and irregular complex) yielded similar conformity for spherical lesions for both techniques (Chapter 4). For complex and irregular lesions, if the target was in close proximity to critical structures protons seemed to be potentially better. In any case, because of their nature, protons always resulted in a lower integral dose to the brain.

A subset of the lesions from the study discussed in Chapter 3 was taken into a further study for comparison, using the same field set-up and constraints. The question was if further improvement in dose distribution could be achieved with intensity-modulated protons and IMSRT (Chapter 5). The conclusion was, that dose conformation to the PTV was equally good for all 3 techniques. Organs-at-risk situated in concavities (e.g. the brain stem) were equally well spared by IMSRT and by proton beams. The amount of normal tissue irradiated is lowest with IMPT. In contrast with the previous two studies, the addition of intensity modulation improves both modalities of SCRT and protons independently of tumour shape.

The clinical impact of higher conformality needs to be addressed in future studies. At present, it can be said that conformity and reduction of integral dose and dose to critical structures are warranted in first instance when treating benign tumours, paediatric tumours or recurrent pre-irradiated tumours of the brain in order to minimize morbidity.

\section{CLINICAL OUTCOME}

\section{SCRT boost for malignant glioma}

Radiotherapy has been the single most effective treatment for patients with malignant glioma with survival benefit proven in prospective randomised clinical studies $^{49,50}$. These studies have proved the existence of a dose response relationship in survival time for patients receiving doses $>60$ Gy compared to those receiving doses of 55 and $50 \mathrm{~Gy}$. However, tumours still recur at the primary site despite local radical 
irradiation to the level of radiation tolerance of $60 \mathrm{~Gy}$. It therefore seemed logical to exploit conformal radiotherapy techniques such as SCRT for local dose escalation in order to improve tumour control and survival without increasing late normal tissue damage. To determine the value of an additional fractionated stereotactic boost after conventional radiotherapy as used in the joint randomised EORTC22972/ MRC-BR 10 study $^{3}$, we conducted a prospective single-arm study in patients with a small malignant glioma $(\leq 4 \mathrm{~cm})($ Chapter 6$)$. Compared to a historical group of patients with a small glioblastoma multiforme irradiated with an equivalent total dose of 60 Gy without stereotactic boost, overall survival was significantly better for patients irradiated with additional SCRT boost to a total dose of $80 \mathrm{~Gy}$. One reason for this may be patient selection and the small number of patients in the study. In the meantime a first report on the (closed) RTOG 9305 trial has been published in abstract form ${ }^{46}$. In this trial, patients were randomised between conventional radiotherapy to 60 Gy and SRS followed by conventional radiotherapy. There was no difference in survival between the two arms (median survival 14.1 and 13.7 months). In a subgroup analysis of patients with a pre-operative tumour size not larger than $4 \mathrm{~cm}$ a trend towards an improvement in the SRS arm was seen. However, this was not an endpoint of the study. The publication of all data should bring more clarity to these issues. Toxicity was low in the upfront SRS boost arm. This was also reported in our study where the additional SCRT boost after conventional radiotherapy resulted in radionecrosis in only one patient from 17 . For a subset of patients as defined in Chapter 6 (good clinical condition, small tumours) an additional stereotactic boost should be applied until the full publication of the results or until new data become available.

\section{SCRT for orbital meningioma}

Another example of a seemingly good indication for SCRT is orbital meningioma. In Chapter 7 we report on a multi-centre study with 23 patients accrued between 1996 and 2003 from 4 European Radiation Oncology centres. After a median follow-up of 20 months (1-68 months), we observed a $95 \%$ (21 of 22) visual control rate. Vision improved in 16 patients and remained stable in 5 . The use of radiotherapy for this tumour entity in order to preserve vision has been described as early as 1977: Montgomery and co-authors ${ }^{31}$ have reported on 16 patients treated between 1962 and 1975 . Of these patients, 15 had a histopathologically confirmed diagnosis: in 12 out of 16 patients, vision was preserved or improved. Four patients recurred, all of whom had total doses less than $50 \mathrm{~Gy}^{31}$. These results were confirmed in the early eighties by Smith et a ${ }^{45}$. In $95 \%$ of patients where a worsening of vision has been documented, a control in vision was observed (16 improved, 5 stabilized). This confirms data in the literature: recent studies using SCRT report on a total of 45 patients the same striking functional improvement of $95 \%$ (43/45 cases with 20 eyes having improved vision, 23 being stable) $)^{1,27,42}$. Summarizing all 118 published cases treated 
with irradiation alone (including the investigation of this thesis), the overall visual control rate is $91.5 \%$ (Appendix 1 ). With the advent of modern conformal radiation techniques, there seems to be a promise of less toxicity, although detailed side effects were not reported in older studies (Appendix 1). Lower toxicity was also observed in this multi-centre study: side effects were mild and serious late side effects were reported in only one patient after a follow-up period of 48 months. However, it is remarkable that a useful vision could be maintained for a period of 4 years.

Another most challenging aspect of this study was the observation of an early response to SCRT: the majority occurred within 3 months after the completion of treatment (some even during treatment) and vision did not worsen again later.

In a comparison of an untreated group of patients ( 6 eyes) with those treated by SCRT at Zurich University Hospital (8 eyes) we observed a clearly unfavourable natural course of this disease. When visual acuity at the last follow-up was compared with visual acuity at the time of diagnosis, it was worse almost exclusively in the untreated group ${ }^{25}$. Irradiated patients experienced improvement of visual function in 6 out of 8 eyes ( 1 was already blind). In the untreated group, 4 showed a marked worsening of vision, while 2 eyes remained with a stable visual function ${ }^{25}$.

Based on these facts, and as there is usually no restoration of vision in blind eyes, SCRT can be recommended as the treatment of choice and should be offered after the first documentation of visual deterioration.

\section{Paediatric low-grade gliona}

Radiation therapy is an effective treatment modality in children with low-grade glioma. For many years now it has been an integral part in the management of this disease in children. Low-grade gliomas carry an excellent long-term prognosis. Thus, long-term side effects are therefore of particular concern in children. Radiation-induced late toxicity can be divided into three major groups: neurocognitive dysfunction, endocrine dysfunction and growth retardation, vasculopathies and secondary malignant tumours arising within the irradiated area. A high precision irradiation technique such as SCRT allows the reduction of safety margins around the tumour and this leads to a reduction of the volume of normal brain tissue irradiated. In turn, this should result in reduced long-term toxicity. However, a reduction of the safety margin may carry the risk of reduced local tumour control. The comparison of two groups of children harbouring a low-grade glioma treated either with fractionated SCRT $(n=14)$ and a historical cohort $(n=33)$ treated with conventional radiotherapy to the same dose is reported in Chapter 8. After a median follow-up of 33 months the local control for the SCRT group was the same as for the historical con- 
trol group of patients treated with conventional external beam RT. Toxicity was comparable, two children experienced new endocrine dysfunction after SCRT. The frequency of delayed hypothalamic-pituitary axis dysfunction reflects tumour location adjacent to the hypothalamus and pituitary. Based on preliminary data reported by Merchant and colleagues ${ }^{28}$, for the 3D-CRT technique a margin of $10 \mathrm{~mm}$ for CTV plus $5 \mathrm{~mm}$ for PTV appeared to be safe for paediatric patients with low-grade glioma. However, as shown in our study, it seems possible to reduce the safety margin even further to 5-10 mm and still maintain the same tumour control. Normal tissue sparing through the use of SCRT should be beneficial to paediatric patients if the rate and patterns of failure are similar to conventional techniques and toxicity reduction can be objectively documented. However, for a definite conclusion longer follow-up is required.

\section{CONCLUSIONS}

The reproducibility of patient positioning using a relocatable stereotactic head mask system combined with a bite-block is significantly higher than combined with a simple upper-jaw support. Thus, in order to further reduce the margin for the planning target volume a dental fixation is necessary.

The comparison between different high-precision 3D CRT techniques indicates that intensity modulation of both SCRT and protons can yield very similar dose distributions that closely conform to the three-dimensional shape of the target volume while minimizing dose to organs-at-risk in defined situations. For concave and complex brain lesions a higher level of conformality of dose was achieved with IMSRT than with SCRT. A similar comment applies to IMPT and protons. Normal structures lying in a cavity were better spared with IMSRT and both proton techniques. The advantage of protons and to a greater effect of IMPT is the lower integral dose. The use of sophisticated planning and highly precise treatment modalities should first be considered in children and when tumours carry an excellent long-term prognosis (as benign brain lesions).

From a clinical point of view the use of SCRT is promising. Tumour control and an improvement or stabilisation of symptoms can be maintained with a smaller volume irradiated (as shown here for paediatric low grade glioma and optic nerve sheath meningioma). The reduction of long-term sequelae needs to be demonstrated after a longer follow-up period.

The question of dose escalation for patients with a malignant glioma needs to be addressed in different settings, for example in combination with radiosensitizing 
drugs or cytotoxic therapies administered together with radiotherapy. A first step in this direction was taken in the randomised EORTC and NCIC study with concomitant and adjuvant chemotherapy in the form of temozolomide. The combined treated group of patients had a significant survival advantage compared to patients treated with radiotherapy alone ${ }^{47}$. The sole use of an additional stereotactic boost after conventional irradiation does not seem to be a standard therapy for the majority of patients with a malignant glioma, but may be an option for a well-defined subgroup.

\section{FUTURE PERSPECTIVES}

\section{Image guided radiotherapy}

The future will see further technical developments for highly conformal radiotherapy and treatment planning. The coming years will bring exciting tools to the clinic, such as sophisticated planning systems using biological data from functional imaging (e.g. PET or MR-spectroscopy) for target volume definitions, planning algorithms based on biological aspects of the tumour, on TCP/NTCP, frameless stereotactic treatment and frameless image fusion systems. Accent will be placed on soft tissue imaging rather than using anatomical references to "see and treat" the tumour. The $4^{\text {th }}$ dimension, namely organ and tumour motion will be integrated into treatment planning including its effect on DVH calculation and thus 3D-CRT will develop into 4D-CRT. The use of continuous on-line adjustment of the patient's position will enable corrections for intra- and inter-fraction motions. One of the simplest variants of image guided radiotherapy (IGRT) is portal imaging based, where daily measurements of defined structures can be used as criteria for on-line corrections ${ }^{6}$. More sophisticated systems with automatic patient and target repositioning make use, for example, of imaging devices placed in the treatment room. Conventional CT scanners, ultrasound devices, or cone beam CT (using either an X-ray unit mounted directly on the accelerator gantry, or the accelerator beam, or both, for simultaneous imaging) have been used to visualise the tumour prior to treatment. This makes possible an on-line correction of the alignment of the planned and treatment isocentre and of target volume deformation and tumour shrinkage with dose on a regular basis.

\section{Future clinical trials}

The rapid technical development carries the risk of a hurried introduction of new techniques into the clinic and its application as a standard treatment without first being thoroughly tested. Proper testing and search for indication for each new tech- 
nique sometimes needs a longer gestation period than for developing it. In this light stereotactic radiotherapy as such is already an "old" treatment technique, but its theoretical advantage in the reduction of long-term toxicity still needs to be proven. Long-term follow-up and clinical studies with carefully selected end-points not only for tumour control but also for the preservation of the function of normal tissue are required. There is a lack of Level 1 evidence for stereotactic radiotherapy indications mainly due to the small number of centres using SCRT. For this reason an EORTC randomized study for dose escalation with a SCRT boost for patients with malignant glioma had to be closed due to lack of accrual. In view of the increasing number of centres in the Netherlands starting to use SRT techniques, a national working group for SRT will be founded for co-operation in randomized clinical trials addressing specific SCRT questions.

\section{Target volume definition}

An important aspect of highly conformal treatment is proper target volume definition. An inaccurately defined target volume may render an accurate treatment less effective. For example, there is no consensus on the definition of the CTV in radiosurgery of brain metastases. Many centres include the contrast-enhancing tumour only ${ }^{5,11}$; some add a margin of $1-2 \mathrm{~mm}^{7,9,36,40}$. While metastases are well circumscribed on imaging, their shape may vary and the possible tumour spread outside of the enhancing area is not well known. The question of whether there is a need or not for an additional margin around the contrast-enhancing lesion in order to define the CTV for radiosurgery of brain metastases is currently being investigated.

\section{Biological optimisation}

Dose distributions based on biological effect is more difficult to calculate than purely physical dose distribution based on intensity modulation and advanced algorithms, which include corrections for motion. For new generation TPS, dose calculations based on biological modelling the time factor plays as important a role as clinical data. For example, in the Department of Radiation Oncology Maastricht a study of patients diagnosed with a primary brain tumour (glioma or meningioma) has been initiated, where blood samples and tumour tissue taken before the start of radiotherapy are collected in a biobank in order to relate side-effects and treatment outcome with polymorphisms in the genetic code (i.e. a molecular data base). The aim is to gain insight into molecular characteristics of the tumour and normal tissue and evaluate response to treatment. Based on these data the risk of failure of standard therapy or the risk of developing severe side effects can be estimated. The most challenging results may come from the combination of these molecular data with prospective databases containing radiotherapy volume data (detailed 3D dose distributions, 
NTCP, TCP data), imaging data and clinical outcome data making it possible to correlate results with the molecular database. Until definite data become available, methodological studies following those of Chapter 3 to 5 comparing NTCP based planning and radiobiologic modelling versus intensity modulated plans for different target shapes may provide further knowledge in order to help choose the best treatment modality.

\section{Functional imaging}

Molecular and imaging data are combined in functional imaging. In MR spectroscopy, molecules defining a tumour or infiltration zone and molecules, which are more specific to normal tissue, can be identified (for example, $\mathrm{N}$-acetyl aspartate as a specific neuronal marker of normal brain tissue). This information can be used for radiotherapy planning as well as for follow-up imaging. Choline is often the dominant metabolite to reflect tumour changes in MR imaging ${ }^{35}$. After serial MRS in patients with a low-grade glioma a significant reduction in the mean choline signal was observed, which paralleled the reduction in tumour volume ${ }^{33}$. The first exploration in radiotherapy planning in low and high-grade gliomas showed metabolically active tumour beyond the contrast enhanced or hyper-intensity areas in a limited and non-uniform fashion. The resulting CTV could be reduced by using spectral data ${ }^{35,41}$. On the other hand, the CTV increased by using ${ }^{123}$ I- $\alpha$-methyl-tyrosine SPECT, but as for MRS based planning, abnormalities differed considerably from those found on $\mathrm{MRI}^{17}$. Radiotherapy planning with PET is another promising area of research. PET scanning with 18-FDG is difficult to interpret due to the high uptake of FDG in the normal brain tissue and RT planning may be especially hampered by this fact ${ }^{16}$. However, changes in regional FDG uptake of serial FDG PET studies in high-grade gliomas during and after $\mathrm{RT}$ could be assessed with a different method of pixel-wise regression analysis (Figure 1, own unpublished data). This is especially challenging in brain tumours, where the tumour often cannot be radically resected. The combination of new tracers, new methods of image analysis and the use of a combined PET-CT data will increase the sensitivity for tumour definition and thus the reduction in treatment volumes, and opens a wide area for future clinical studies.

\section{REDUCTION OF INTEGRAL DOSE}

A clinically significant increase in the efficacy of radiotherapy will therefore be derived from a more conformal or closer approximation of the treatment volume to the defined target and by differentiation of tissue response between tumour and normal structures. There is little doubt that highly accurate $3 \mathrm{D}$ conformal radiotherapy 

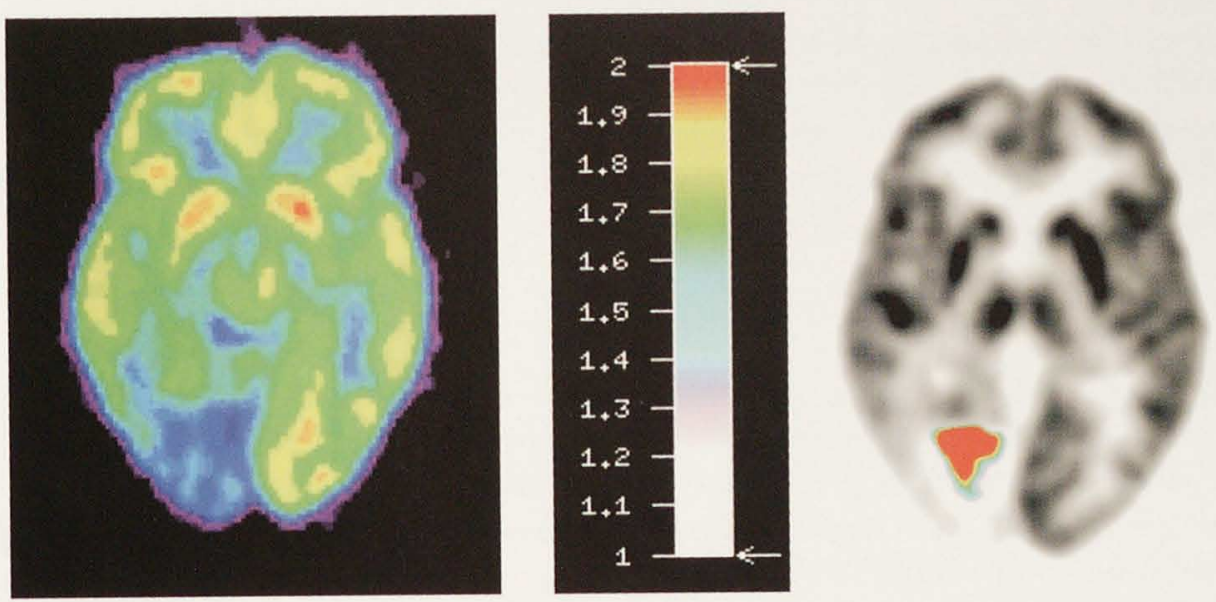

FDG uptake in tumoral ROI

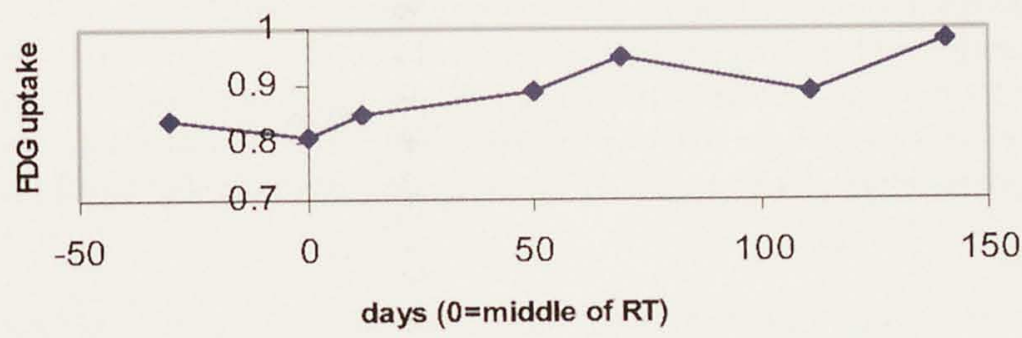

Figure 1. FDG PET shows FDG uptake in the surgical cavity (left top). Image demonstrating tumour recurrence by filling of the surgical cavity (middle and right top). Semi-quantitative ROI analysis suggesting tumour progression because of a slowly rising time regression curve (bottom).

techniques are becoming standard practice. The question as to which technique should be chosen will likely be decided on the basis of cost-benefit analyses as long as no additional clinical and/or biological data are available for a more differentiated decision-making process. Additionally, the risk of a radiation-induced second cancer must be taken into account. The risk of a second malignancy in patients treated with IMR T due to an increase in the integral dose is estimated to be approximately double that for conventional radiotherapy (from $1 \%$ to $1.75 \%$ ) for patients surviving 10 years ${ }^{18}$. This would be a major issue only for patients with a benign tumour and a good prognosis and should not prevent the use of photon IMRT. In this respect irradiation with charged particles as protons or light ions could be an ideal solution because of their LET characteristics and their penetration in matter without an exit 
dose. The combination of stereotactic radiosurgery and proton beam techniques in the form of IMPT could therefore make the ultimate reduction in treatment volume possible. If these are combined with radiobiologically optimised dose calculation and delivery techniques treatment outcome for patients with advanced tumours may be improved by $10-40 \%{ }^{4}$.

\section{Radiation sensitizers}

Last but equally important is the constantly evolving area of combined treatment with radiosensitizing or cytotoxic drugs or biologic modifiers. The most recent and fascinating results in this area are those of Gefinitib (Iressa), an epidernal growth factor receptor (EGFR) tyrosine kinase inhibitor. Following the observation that response to treatment with Genifitib was more pronounced in specific patient groups such as Japanese patients, women and non-smokers ${ }^{12,20,24,30}$, the molecular basis of this sensitivity has been examined. It seems that a mutation of the receptor tyrosine kinases is associated with a higher sensitivity to Gefinitib ${ }^{37,38}$ and that patients harbouring this specific EGFR mutation should therefore be the subject of future clinical trials with EGFR inhibitors. This not only applies to patients with lung cancer but also to patients with glioblastoma where the existence of EGFR alterations has been demonstrated ${ }^{52}$. Individualized treatment based on pre-treatment profiling with genetic or other high throughput assays will be part of future trials. Furthermore, the potential of Gefinitib in radiosensitizing glioblastoma to radiation has been reported ${ }^{2}$.

Given the poor prognosis of patients with high-grade gliomas and the wide gap between various therapies tried (by means of dose-escalation or drugs) and the poor treatment response, further therapeutic improvement will remain a most challenging topic for the future. This is of special interest as for this tumour entity the therapeutic gain has, until recently, been very small. Results of the joint EORTC-NCIC phase III study randomising between radiotherapy alone and combined concomitant and adjuvant temozolomide therapy showed that patients having received the combined treatment had a significantly better median and 2-year survival (from 8 to $24 \%)^{47}$. The next step to further improve survival for this patient group would be the addition of biological modifying and radiosensitizing drugs. One of these could be cyclo-oxygenase-2 (COX-2) inhibitor. COX-2 is an enzyme induced by a variety of factors including tumour promoters, growth factors and hypoxia. Its over-expression is linked to carcinogenesis, tumour growth, angiogenesis and the facilitation of metastatic spread. A higher COX-2-expression is associated with clinically more aggressive gliomas and thus a prediction of poorer survival ${ }^{44}$. Early studies have shown that COX-2 inhibition may improve glial tumour response to radiation'22,29,39,43. There- 
fore, selective COX-2 inhibitors represent a potential molecular target in the treatment of brain tumours.

Beside EGFR inhibitors and COX-2 inhibitors, mTOR inhibitors (e.g. rapamycin) seem to be another promising biological modifier and radiosensitizer for glioma patients. Anti-proliferative and anti-angiogenic properties in tumours and cell lines have been shown. The mTOR kinase transduces proliferation, growth and survival signals from many receptor tyrosine kinases (e.g. EGFR). As with Genifitib a synergistic effect of rapamycin with radiation on tumour control has been shown ${ }^{8}$.

Thus, upcoming clinical trials will have not only to include conformal treatment techniques, but also, beside classical clinical endpoints (survival, progression), biological endpoints, targeted therapy and translational research. Some of this will be realized in the near future: the upcoming randomised phase III EORTC trial for patients with low grade glioma will be based on an upfront genetic testing of defined genetic changes, according to which patients will be stratified. Biological endpoints will be correlated with response to both treatment arms as well as to survival. Furthermore, patients will be asked upfront to consent to a prospective tumour tissue banking for later translational research. Translational research will also be integrated in the new EORTC studies for patients with a meningioma, where conformal treatment techniques as stereotactic radiotherapy, radiosurgery and proton therapy can be applied. The correlation of biological endpoints with eventual differences in outcome of highly accurate radiotherapy treatment techniques may bring new insights into TCP and NTCP.

In summary, the future will be conformal, both on a physical and a molecular level.

\section{REFERENCES}

1. Andrews DW, Faroozan R, Yang BP et al. Fractionated stereotactic radiotherapy for the treatment of optic nerve sheath meningiomas: Preliminary observations of 33 optic nerves in 30 patients with historical comparison to observation with or without prior surgery. Neurosurg 2002;51:890-904.

2. Baldassarre $\mathrm{S}$, Falsey $\mathrm{R}$, Kislin $\mathrm{K}$ et al. Time and dose dependent radiosensitization of the glioblastoma multiforme $\mathrm{U} 251$ cells by the EGF receptor tyrosine kinase inhibitor D 1839 ("Iressa"). Cancer Letters 2003;202:43-51. 
3. Brada $M$ and Baumert $B$. Focal fractionated conformal stereotactic boost following radiotherapy of high-grade gliomas: A randomized phase III study. A joint study of the EORTC (22972) and the MIRC (BR10). In: Wiegel, Hinkelbein W, Brock M, Hoell T (eds), Controversies in Neuro-Oncology. Front Radiat Ther Oncol. Basel, Karger, 1999; vol. 33, pp. 241-243.

4. Brahme A. Recent advances in light ion radiation therapy. Int J Radiat Oncol Biol Plys 2004;58(2):603-616.

5. Breneman JC, Warnick RE, Albright, et al. Stereotactic radisourgery for the treatment of brain metastases. Cancer 1997;79:551-557.

6. Ciernik IF, Baumert BG, Egli P and Lütolf UM. On-Line correction of beam portals in the treatment of prostate cancer using an endorectal balloon device. Radiother Oncol 2002; 65(1):39-45.

7. Engenhart R. Kimmig BN, Hover K, ct al. Long-term follow-up for brain metastases treated by percutaneous single high-dose radiation. Cancer 1993;71:1353-1361.

8. Eshleman JS, Carlson BL, Mladek AC, Kastner BD, Shide KL and Sarkaria JN. Inhibition of the Mammalian Target of Rapamycin Sensitizes U87 Xenografts to Fractionated Radiation Therapy. Cancer Research 2002;62,7291-7297.

9. Feuvret L, Germain I, Cornu P, ct al. Traitement premier des metastases cerebrales par irradiation en condition stereotaxique. Bull Cancer 1999;86:666-671.

10. Fineman MS and Augsburger JJ. A new approach to an old problem. Surv Ophthalmol 1999; 43(6):519-524.

11. Flickinger JC, Kondziolka D, Lunsford LD, et al. A multi-institutional experience with stereotactic radiosurgery for solitary brain metastasis. Int J Radiat Oncol Biol Phys 1994;28: 797-780.

12. Fukuoka M, Yano S, Giaccone G, ct al. Multi-institutional randomized phase II trial of gefitinib for previously treated patients with advanced non-small-cell lung cancer. $J$ Clin Oncol 2003;21(12):2237-2246.

13. Goh KY andYeow YK. Optic nerve sheath meningioma-report of three cases. Ann Acad Med Singapore 1994; 23(3):406-411.

14. Grant W III and Cain RB. Intensity modulated conformal therapy for intracranial lesions. Med Dosim 1998; 23(3):237-241.

15. Gregor A, Cull A, Traynor E, et al. Neuropsychometric evaluation of long-term survivors of adult brain tumours: relationship with tumour and treatment parameters. Radiother Oncol 1996;41(1):55-59.

16. Gross $M W$, Weber WA, Feldmann HJ, Bartenstein $P$, Schwaiger $M$ and Molls $M$. The value of F-18-Fluorodeoxyglucose PET for the 3-D radiation treatment planning of malignant glioma. Int J Radiat Oricol Biol Pliys 1998;41(5):989-995.

17. Grosu A, Feldmann HJ, Dick S, et al. Implications of IMT-SPECT for postoperative radiotherapy planning in patients with gliomas. Int J Radiat Oncol Plys 2002;54(3):842-854.

18. Hall EJ and Wuu CS. Radiation-induced second cancers: the impact of 3D-CRT and IMRT. Int J Radiat Oncol Plys 2003; 56(1):83-88. 
19. Ito $M$, Ishizawa $A$, Miyaoka $M$, Sato $K$ and Ishii S. Intraorbital meningiomas: Surgical management and role of radiation therapy. Surg Ncurol 1988; 103:59-60.

20. Janne PA, Gurubhagavatula S, Yeap BY, et al. Outcomes of patients with advanced non-small cell lung cancer treated with gefitinib (ZD1839, "Iressa") on an expanded access study. Luing Caicer 2004;44(2):221-30.

21. Kennerdell JS, Maroon JC, Malton M and Warren FA. The management of optic nerve sheath meningiomas. Am J Ophthalmol 1988;106:450-457.

22. Kishi K, Petersen S, Petersen C, ct al. Preferential enhancement of tumor radioresponse by a cyclooxygenase-2 inhibitor. Cancer Res 2000;60(5):1326-31.

23. Klink DF, Miller NR and Williams J. Preservation of residual vision 2 years after stereotactic radiosurgery for a presumed optic nerve sheath meningioma. J Neuroophthalmol 1998; $18(2): 117-120$

24. Kris MG, Natale RB, Herbst RS, et al. Efficacy of gefitinib, an inhibitor of the epidermal growth factor receptor tyrosine kinase, in symptomatic patients with non-small cell lung cancer: a randomized trial. JAMA 2003;290(16):2149-58.

25. Landert M, Baumert BG, Luetolf UM, Boesch MM and Landau K. Outcome of optic nerve sheath meningioma treated by fractionated stereotactic radiotherapy. Submitted for publication Ncuroophthalmology 2004

26. Lee AG, Woo SY, Miller NR et al. Improvement in visual function in an eye with a presumed optic nerve sheath meningioma after treatment with three-dimensional conformal radiation therapy. J Neuroophthalmol 1996; 16(4):247-251

27. Liu JK, Forman S, Hershewe GL, Moorthy CR and Benzil DL. Optic nerve sheath meningiomas: visual improvement after stereotactic radiotherapy. Neurosurg 2002;50: 950-957.

28. Merchant TE, Zhu Y, Thompson SJ, Sontag MR, Heideman RL and Kun LE. Preliminary results from a Phase II trial of conformal radiation therapy for pediatric patients with localised low-grade astrocytoma and ependymoma. Int J Radiat Oncol Biol Plys 2002 Feb 1;52(2): 325-32.

29. Milas L, Kishi K, Hunter N, Mason K, Masferrer JL and Tofilon PJ. Enhancement of tumor response to gamma-radiation by an inhibitor of cyclooxygenase-2 enzyme. J Natl Cancer Inst 1999;91(17):1501-4.

30. Miller VA, Kris MG, Shah N, et al. Bronchioloalveolar pathologic subtype and smoking history predict sensitivity to gefitinib in advanced non-small-cell lung cancer. J Clin Oncol 2004;22(6):1103-9.

31. Montgomery AB, Griffin T, Parker RG and Gerdes AJ. Optic nerve glioma: the role of radiation therapy. Cancer 1977; 40(5):2079-2080.

32. Moyer PD, Golnik KC and Breneman J. Treatment of optic nerve sheath meningioma with three-dimensional conformal radiation. Am J Ophthalmol 2000; 129(5):694-696.

33. Murphy PS, Vivier L, Abson C, Rowland IJ, Brada M, Leach MO and Dzik-Jurasz ASK. Monitoring temozolomide treatment of low-grade glioma with proton magnetic resonance spectroscopy. British J Cancer 2004;90:781 - 786. 
34. Narayan S, Comblath WT, Sandler HM, Elner V and Hayman JA. Preliminary visual outcome after three-dimensional conformal radiation therapy for optic nerve sheath meningioma. Int J Radiat Oncol Biol Phys 2003;56:537-543.

35. Nelson SJ, Graves E, Pirzkall A, et al. In vivo molecular imaging for planning radiation therapy of gliomas: an application of 1 H MR TSI. JMagn Reson Imaging 2002;16:464-476.

36. Noel G, Simon JM, Valery CA, et al. Radiosurgery for brain metastasis: impact of CTV on local control. Radiother Oncol 2003;68(1):15-21.

37. Ono M, Hirata A, Kometani T, ct al. Sensitivity to gefinitib (Iressa, ZD 1839) in non-small cell lung cancer cell lines correlates with dependance on the epidermal growth factor (EGF) receptor/extracellular signal-regulated kinase 1/ 2 and EGF receptor/Akt pathway for proliferation. Mol Cancer Ther 2004;3(4):465-472.

38. Paez Jg, Jänne PA, Lee JC, ct al. EGFR mutations in lung cancer: Correlation with clinical response to therapy. Scicnccexpress 2004;1-8

39. Petersen C, Petersen S, Milas L, Lang FF and Tofilon PJ. Enhancement of intrinsic tumor cell radiosensitivity induced by a selective cyclooxygenase- 2 inhibitor. Clin Cancer Res 2000 ;6(6):2513-20.

40. Pirzkall A, Debus J, Lohr F, et al. Radiosurgery alone or in combination with whole-brain radiotherapy for brain metastasis. J Clin Oncol 1998;16:3663-3669.

41. Pirzkall A, Nelson SJ, McKnight T, et al. Metabolic imaging of low.grade gliomas with three-dimensional magnetic resonance spectrosopy. Int J Radiat Oncol Pliys 2002;53(5): 1254-1264.

42. Pitz S, Becker G, Schiefer U, et al. Stereotactic fractionated irradiation of optic nerve sheath meningioma: a new treatment alternative. $\mathrm{Br} J$ Ophthalmol 2002;86:1265-1268.

43. Pyo H., Choy H., Amorino GP, et al. A selective cyclooxygenase-2 inhibitor, NS-398, enhances the effect of radiation in vitro and in vivo preferentially on the cells that express cyclooxygenase-2. Clin Cancer Res 2001;7:2998-3005.

44. Shono T, Tofilon PJ, BrunerJM, Owolabi, O and Lang FF. Cyclooxygenase-2 expression in human gliomas: prognostic significance and molecular correlations. Cancer Res 2001;61: 4375-43

45. Smith JL, Vuksanovic MM, Yates BM and Bienfang DC. Radiation therapy for primary optic nerve meningioma. J Clin Neuroophthalmol 1981; 1(2):85-99.

46. Souhani L and Scott C. Randomized Prospective Comparison Of Stereotactic Radiosurgery (SRS) Followed By Conventional Radiotherapy (RT) With BCNU To RT With BCNU Alone For Selected Patients With Supratentorial Glioblastoma Multiforme (GBM): Report of RTOG 93-05 Protocol. Proc Am Soc Ther Rad Oncol (ASTRO) 2002, pp. 94-95.

47. Stupp R, Mason W, van den Bent $M$, ct al. Concomitant and adjuvant temozolomide (TMZ) and radiotherapy (RT) for newly diagnosed glioblastoma multiforme (GBM). Conclusive results of a randomized phase III trial by the EORTC and NCIC Clinical Trial Group. Proc Am Soc Clin Oncol 2004;23:1a (abstract 2) 
48. Thornton Jr. AF, Hegarty TJ, Ten Haken Rk, ct al. Three-dimensional treatment planning of astrocytomas: A dosimetric study of cerebral irradiation. Int J Radiat Oncol Biol Phys 1991;20:1309-1315.

49. Walker MD, Green SB, Byar DP, et al. Randomized comparisons of radiotherapy and nitrosoureas for the treatment of malignant glioma after surgery. N Eng J Med 1980;303: 1323-1329.

50. Walker MD, Strike TA and Sheline GE. An analysis of dose-effect relationship in the radiotherapy of malignant gliomas. Int J Radiat Oncol Biol Plys 1979;5:1725-1731.

51. Webb St: Preface. In: Webb ST (eds). The Physics of Three-Dimensional Radiation Therapy. Conformal Radiotherapy, Radiosurgery and Treatment Planning. Bristol and Philadelphia: Institute of Physics Publishing; 1993 .pp. ix-xi.

52. Yamazaki H, Fukui $Y$, Ueyama $Y$, ct al. Amplification of the structurally and functionally altered epidermal growth factor receptor gene (c-erbB) in human brain tumors. Mol Cell Biol $1988 ; 8: 1816-1820$ 


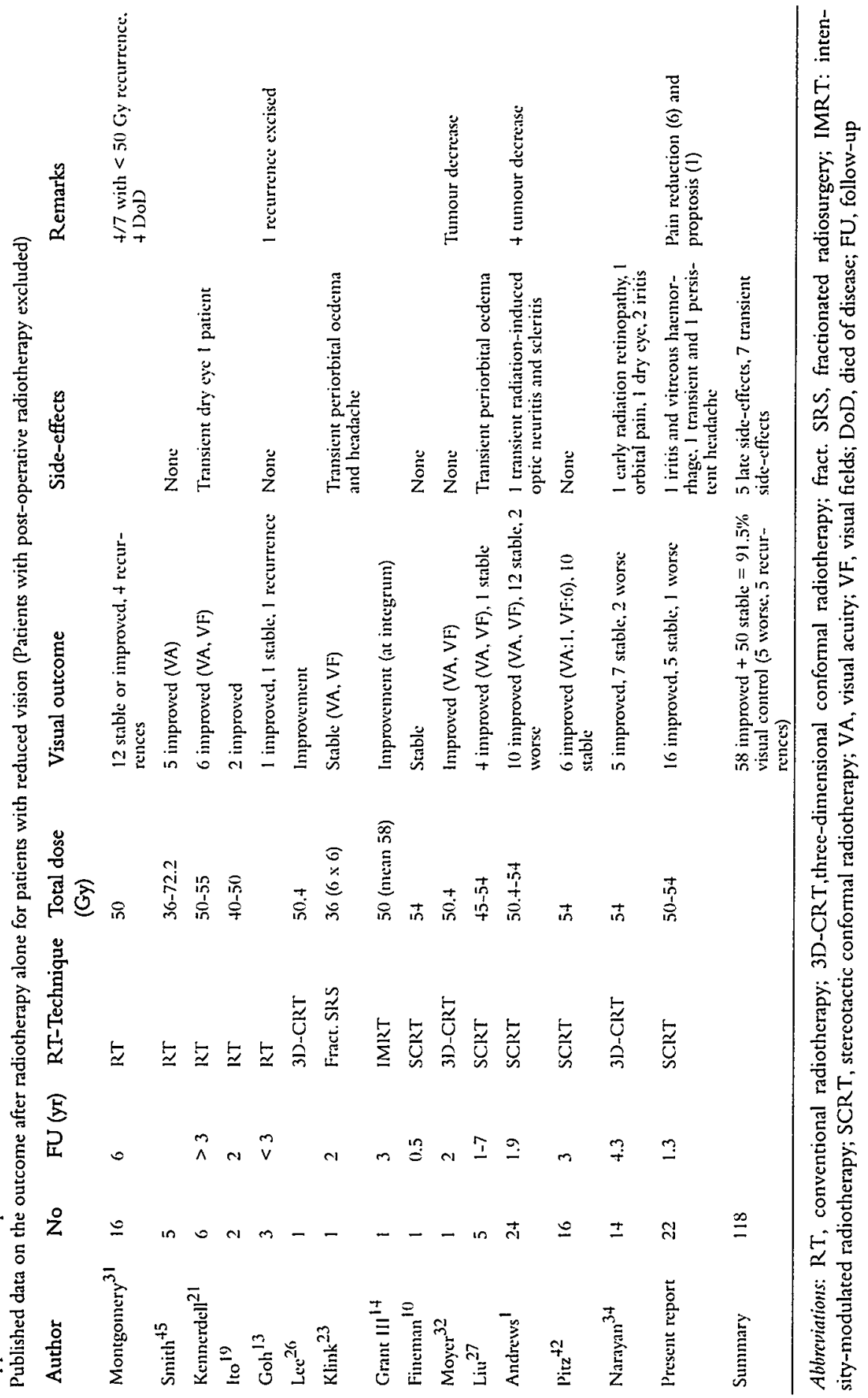




\section{SAMENVATTING}

In Hoofdstuk 1 worden de achtergrond en het doel van de 3D conformatie radiotherapie (3D-CRT) gedefinieerd. De afgelopen 2 decades hebben een snelle en indrukwekkende ontwikkeling van computer technologie laten zien, welke hun intrede hebben gedaan in de radiotherapie. Een belangrijk gevolg is de ontwikkeling van 3D conformatie technieken in de behandeling van kwaadaardige aandoeningen. Instrumenten als computer gestuurde lineaire versnellers en behandeling, 3D treatment planning, multi-leaf collimators, 3D multi-modality beeldvorming en intensiteitsmodulatie hebben een grote bijdrage aan de technische vooruitgang van de radiotherapie geleverd. Gebruik hiervan resulteerde in 3D conformatie therapie, die gedefinieerd kan worden als het gelijkvormig maken van het bestralingsvolume aan het behandelingsvolume in alle drie dimensies. Een andere recente ontwikkeling is modulatie van de intensiteit van de bestralingsbundel, ook wel intensiteitgemoduleerde radiotherapie (IMRT) genoemd. Deze techniek maakt modulatie van de dosis in het behandelingsvolume mogelijk en leidt tot een betere sparing van het omliggende normale weefsel dan met conventionele technieken. Aangenomen wordt, dat deze techniek de kans op genezing zal doen toenemen met afname van de lange termijn toxiciteit, door een hogere dosis toe te dienen in kleinere volumina. Dit is belangrijk in de behandeling van tumoren van het centraal zenuwenstelsel (CZS) waar radiotherapie een belangrijk onderdeel van de behandelingsstrategie vormt.

Het proces van conformatie radiotherapie bestaat uit meerdere stappen die sterk van elkaar afhankelijk zijn. De behandelingsvoorbereiding en bestraling zelf van 3D-CRT kan vergeleken worden met een ketting waarvan de sterkte bepaald wordt door de zwakste schakel. De onderdelen van de keten worden beschreven in Hoofdstuk 1.

De doelen van deze dissertatie worden in detail beschreven aan het eind van Hoofdstuk 1, paragraaf 1.9, en betreffen de voorwaarden waaraan stereotactische conformatie radiotherapie (SCRT) moet voldoen, zoals nauwkeurigheid van het systeem, onderzoek naar voor- en nadelen van verschillende bestralingstechnieken met 3D-CRT met het oog op conformaliteit, het besparen van kritieke organen en klinische resultaten van SCRT voor verschillende intracraniële aandoeningen.

Hoofdstuk 2 beschrijft de nauwkeurigheid van verschillende fixatie methoden gebruikt voor gefractioneerde stereotactische radiotherapie. Grote nauwkeurigheid 
is een voorwaarde voor SCRT voor het behandelen en het sparen van normale weefsels. We hebben drie verschillende fixatie methoden geëvalueerd: een stereotactisch masker met en zonder bite-block of met fixatie van de bovenkaak alleen. Alle 3 vormen van fixatie zijn geëvalueerd door middel van achtereenvolgende $C T$ scans. De nauwkeurigheid van repositionering van de patient is onderzocht door het bepalen van de plaats van het isocentrum in relatie tot de anatomie. Dit werd gemeten door de positie van het isocentrum op de planning CT te vergelijken met die op de controle CT. Honderdveertien controle CT's van 57 patiënten zijn geëvalueerd. Er bleek een significante verbetering van de $3 \mathrm{D}$ vector en de cranio-caudale deviatie bij gebruik van het bite-block in vergelijking met de fixatie van de bovenkaak. Het toevoegen van de bovenkaak fixatie reduceerde significant de laterale rotatie van het hoofd in vergelijking met het masker alleen. Om een zo groot mogelijke winst in reductie van de marge en dus een reductie van hoeveelheid bestraald normaal weefsel te bereiken, is de gebruik van een individueel bite-block noodzakelijk.

In de Hoofdstukken 3 tot 5 word de hypothese onderzocht of de dosisverdeling met het oog op conformaliteit van SCRT kan worden verbeterd door gebruik te maken van intensiteitsmodulatie of protonen. Verbetering zal mogelijk leiden tot een verbetering van de therapeutische ratio door reductie van de dosis in de normale weefsels met afname van het risico van morbiditeit. Voor alle 3 studies werden patiënten geselecteerd, die behandeld waren met SCRT en opnieuw gepland voor de vergelijking. Verder werden voornamelijk tumoren, gelokaliseerd in de schedelbasis gekozen, omdat deze meestal door meerdere kritische structuren omgeven zijn en vaak een complexe en onregelmatige vorm hebben. Voor de volledigheid zijn er ook tumoren met multifocale en ovale vormen in de studie opgenomen. Conformaliteit voor SCRT werd bereikt door gebruik te maken van 4 tot 6 niet coplanaire bundels. Bij intensiteitsgemoduleerde inverse plannings technieken werd gebruik gemaakt van speciale optimalisatie algoritmes, die kritieke organen sparen door toepassing van bepaalde dosis voorwaarden (constraints) en gewichtsfactoren. Bij protonen werd gebruik gemaakt van de spot-scanning techniek en energie modulatie.

In de eerste studie wordt de toevoeging van IMRT aan SCRT onderzocht (Hoofdstuk 3). Tien patiënten (7 met een schedelbasismeningeoom) werden hiervoor geselecteerd. Aangetoond werd een betere conformaliteit voor intensiteitsgemoduleerde stereotactische radiotherapie (IMSRT) voor onregelmatige en multifocale tumoren. Sparing van kritieke organen, vooral indien gelegen in een concaviteit, was beter met IMSR. Echter, het volume van normaal weefsel dat een lage dosis ontving ( $=30 \%$ isodose) was groter in vergelijking met IMSRT in 6 van de 10 gevallen. 
De vergelijking van SCRT en protonen van 7 laesies, onderverdeeld naar vorm (concaaf, ovaal, oppervlakkig en dicht bij kritieke organen, irregulier en complex) liet een gelijke conformaliteit zien (Hoofdstuk 4). Echter voor complexe en onregelmatige laesies, waarbij het doelvolume zich dicht bij kritische structuren bevindt lijkt behandeling met protonen beter. In alle gevallen lijkt behandeling met protonen, op basis van de fysische eigenschappen, te resulteren in een lagere integrale dosis in de hersenen.

Een deel van de laesies, zoals beschreven in Hoofdstuk 3, werd gebruikt voor vergelijking in een vervolgstudie, uitgaande van dezelfde veld set-up en constraints (Hoofdstuk 5). De vraag was, of een verdere verbetering van de dosisverdeling kan worden bereikt met intensiteitsgemoduleerde protonen (IMPT) en IMSRT. Opnieuw bleek de dosisconformatie om het PTV (planning target volume) even goed voor alledrie de technieken. De kritische organen gelegen in concave ruimtes (bijvoorbeeld de hersenstam) werden in beide gevallen (IMSRT en protonen) even veel gespaard. De hoeveelheid bestraald normaal weefsel was het laagst met IMPT. In tegenstelling tot de twee eerdere studies liet de toevoeging van intensiteitsmodulatie een verbetering zien voor beide methoden, onafhankelijk van de tumor vorm.

Op dit moment, kan worden gesteld, dat conformaliteit, reductie van integrale dosis en dosis in de kritieke organen vooral van belang zijn bij de behandeling van benigne tumoren, tumoren bij kinderen of recidief tumoren na eerdere bestraling met het doel minimaliseren van de morbiditeit.

Om de waarde te bepalen van een aanvullende gefractioneerde stereotactische boost na conventionele radiotherapie, zoals gebruikt in de gerandomiseerde EORTC22972/MRC-BR10 studie, voerden we een prospectieve eenarmige studie uit bij patiënten met kleine hooggradige gliomen (= $4 \mathrm{~cm}$ )(Hoofdstuk 6). Vergeleken met een historische groep bestaande uit patiënten met een klein glioblastoma multiforme bestraald met een gelijke totale dosis van $60 \mathrm{~Gy}$ zonder een stereotactische boost, was de overall survival significant beter voor patiënten bestraald met een SCR T boost tot een totale dosis van $80 \mathrm{~Gy}$. De toxiciteit was laag, bij een patiënt ontstond radionecrose. Vooralsnog lijkt het zinvol aan patiënten die voldoen aan de criteria zoals beschreven in Hoofdstuk 6 (goede klinische conditie, kleine tumoren) een stereotactische boost te geven. Gegevens van verdere studies moeten afgewacht worden.

In Hoofdstuk 7 beschrijven we een multicentrische studie met 23 patiënten met een primair opticus meningeoom, behandeld tussen 1996 en 2003 in 4 deelnemende Europese Radiotherapie centra. Een opticus meningeoom leidt in vrijwel alle gevallen tot blindheid. Alle patiënten zijn behandeld met gefractioneerde stereo- 
tactische bestraling. Na een mediane follow-up van 20 maanden zagen we in $95 \%$ van de gevallen (21 uit 22) verbetering of stabilisatie van de visus. De visus verbeterde bij 16 patiënten en bleef stabiel bij 5 . Dit ondersteunt gegevens uit de literatuur. De toxiciteit was mild en een ernstig laat effect werd gezien in slechts een patiënt, na een follow-up van 48 maanden. Deze werd blind aan het bestraalde oog, na een periode van 4 jaar met een redelijke visus. Een ander opvallende waarneming was de snelheid van klinische respons op SCRT, in de meeste gevallen binnen 3 maanden na behandeling. Dit was een blijvend effect. Op basis van deze gegevens en gezien het feit dat meestal geen herstel van visus optreedt bij blindheid, kan SCRT worden aanbevolen als voorkeurbehandeling en zou gegeven moeten worden na de eerste tekenen van verslechtering van de visus.

De radiotherapie is een effectieve behandeling bij kinderen met een laaggradig glioom. In Hoofdstuk 8 worden 14 kinderen met een laaggradig glioom behandeld met gefractioneerde SCRT en vergeleken met een historische controle groep van 33 kinderen behandeld met conventionele radiotherapie met dezelfde dosis. De locale controle was gelijk voor beide groepen na een mediane follow-up van 33 maanden. De toxiciteit was vergelijkbaar. Twee kinderen ontwikkelden endocriene dysfunctie na SCRT. In onze studie bleek het mogelijk de marge te reduceren tot 5 $\mathrm{mm}$ zonder verlies van tumor controle. SCRT heeft alleen voordeel als het percentage en het patroon van recidiveren gelijk is aan de conventionele bestraling en reductie van toxiciteit objectief kan worden gedocumenteerd. Voor een definitieve conclusie is echter een langere follow-up noodzakelijk. 


\section{CURRICULUM VITAE}

The author was born on $25^{\text {th }}$ of September 1961 in Dortmund, Germany. After primary school she attended high school from 1972 - 1981. She graduated from medical school of the Albert-Ludwig-University in Freiburg in 1981. Before commencing her clinical work she trained for a year in medical computation $1988-1989$. She obtained her MD from the Albert-Ludwig-University Freiburg in 1990.

During her training in radiation oncology, she completed residencies in several disciplines. She trained in radiation oncology at the University Hospital Zurich, Switzerland (Prof. Dr. Urs M. Lütolf) where her interest in stereotactic irradiation started with the introduction of stereotactic radiotherapy. She was engaged in this project and developed it to its full functional level. Specialisation in neuro-oncology and stereotactic radiotherapy followed for which she spent time as a visiting fellow at the Joint Centre for Radiotherapy in Boston, Massachusetts, USA (Prof. Dr. Norman Coleman), and as a clinical research fellow at the Royal Marsden Hospital in London and Sutton, U.K. (Prof. Dr. Michael Brada). From 1997 to 2001 she was staff member in the Clinic for Radiation Oncology in Zurich.

In January 2002 she moved to the Netherlands and is presently a staff member in the Department of Radiation Oncology at the Academic Hospital Maastricht (Prof. Dr. Philippe Lambin). There she initiated stereotactic radiotherapy and is the responsible physician for neuro-oncology. In 2003, she was elected chairman of the Brain Tumour Working Party of the EORTC Radiotherapy Group. She is a member of several professional societies including the EORTC RT and Brain Tumour Group and the EANO (European Association of Neuro-oncology). 


\section{LIST OF PUBLICATIONS}

Koch HK, Baumert B, Koch U, Oehlert M, Oehlert W. Prevalence of campylobacter pylori as demonstrated by histology or CLO-test in different types of gastritis, a study in 5 clinically predefined types of patients. Path Res Pract 1990;186,154-158.

Baumgartner I, von Hochstetter A, Baumert B, Lütolf U, Follath F. Langerhans'-Cell Histiocytosis in Adults. Medical and Pediatric Oncology 1997;28:9-14.

Alheit H, Saran FH, Warrington AP, Rosenberg I, Perks J, Jalali R, Shepherd S, Beardmore C, Baumert B, Brada M. Stereotactically guided conformal radiotherapy for meningiomas. Radiother Oncol 1999;50(2):145-50.

Brada M, Baumert B. Focal fractionated conformal stereotactic boost following radiotherapy of high-grade gliomas: A randomized phase III study. A joint study of the EOR TC (22972) and the MRC (BR 10). In: Wiegel, Hinkelbein W, Brock M, Hoell T (eds), Controversies in Neuro-Oncology. Front Radiat Ther Oncol. Basel, Karger, 1999; vol. 33, pp. 241-243.

Huguenin PU, Taussky D, Moe K, Meister A, Baumert B, Lutolf UM, Glanzmann C: Quality of life in patients cured from a carcinoma of the head and neck by radiotherapy; the importance of the target volume. Int J Radiat Oncol Biol Phys 1999;45(1):47-52.

Huguenin P, Baumert B, Lutolf UM, Wight E, Glanzmann C. Curative radiotherapy in elderly patients with endometrial cancer. Patterns of relapse, toxicity and quality of life. Strahlenther Onkol $1999 ; 175(7): 309-14$

Baumert BG, Lomax AJ, Miltchev V, Davis JB. A comparison of dose distributions of proton and photon beams in stereotactic conformal radiotherapy of brain lesions. Int J Radiat Oncol Biol Phys 2001;49(5):1439-1449.

Jalali R, Loughrey C, Baumert B, Perks J, Warrington AP, Traish D, Ashley S and Brada M. High precision focussed irradiation in the form of fractionated stereotactic conformal radiotherapy (SCRT) for benign meningiomas predominantly in the skull base location. Clin Oncol (R Coll Radiol). 2002;14(2):103-9.

Baumert B, Steinauer K, Lutolf M. Therapie von ZNS-Metastasen. Ther Umsch 1999; $56(6): 338-41$.

Saran FH, Baumert BG, Khoo VS, Adams EJ, Garré ML, Warrington AP and Brada M. Stereotactically Guided Conformal Radiotherapy for Progressive Low Grade Gliomas of Childhood. Int J Radiat Oncol Biol Phys 2002 53(1):43-51.

Ciernik IF, Baumert BG, Egli P, and Luitolf UM. On-Line correction of beam portals in the treatment of prostate cancer using an endorectal balloon device. Radiother Oncol 2002; 65(1):39-45.

Baumert BG, Zagralioglu O, Davis JB, Reiner B, Lütolf UM and Ciernik IF. The Use of a Leg Holder Immobilization in 3D-Conformal Radiation Therapy of Prostate Cancer. Radiother Oncol 2002;65(1):47-52. 
Gruber G, Laedrach K, Baumert B, Caversaccio M, Raveh J, Greiner R. Esthesioneuroblastoma: irradiation alone and surgery alone are not enough. Int $\mathrm{J}$ Radiat Oncol Biol Phys 2002;54(2):486-491.

Dizendorf E, Baumert B, von Schulthess GK, Lïtolf UM, Steinert HC. Impact of ${ }^{18}$ F-Fluorodeoxyglucose Positron Emission Tomography ${ }^{18}$ F-FDG PET on Staging and Managing Patients for Radiation Therapy. Journal Nuclear Medicine 2003 44(1):24-29. Commentary by Hicks RJ and McManus MP:30-2.

Baumert BG, Lutterbach J, Bernays R, Davis JB, Heppner FL. Fractionated Stereotactic Radiotherapy Boost after Post-operative Radiotherapy in high grade Gliomas. Radiother Oncol 2003;67(2): 183-190.

Baumann F, Bjeljac M, Kollias S, Baumert BG, Brandner S, Rousson V, Yonekawa Y, Bernays R. Combined Thalidomide and Temozolomide Treatment in Patients with Glioblastoma Multiforme. Journal of Neuro-Oncology, 2004; 67: 191-200.

Ciernik IF, Dizendorf E, Baumert BG, Reiner B, Burger C, Davis JB, Lütolf UM, Steinert HC, Von Schulthess GK. Radiation Treatment Planning Based On An Integrated Computer-Assisted Positron Emission Tomography (PET/CT): A Feasability Study. Int J Radiat Oncol Biol Phys, 2003; 57(3): 853-8633.

Meier S, Baumert BG, Maier T, Wellis G, Burg G, Seifert B, Dummer R. Survival and prognostic factors in patients with brain metastases from malignant melanoma. Onkologie 2004;27:145-159.

Baumert BG, Norton I and Davis JB. Intensity Modulated Stereotactic Radiotherapy versus Stereotactic Conformal Radiotherapy for the Treatment of Meningioma pre-dominantly located in the skull base. Int J Radiat Oncol Biol Phys 2003; 57(2): 580-592.

Baumert BG, Norton I, Lomax A, Davis JB. Dose conformation of intensity modulated stereotactic photon beams, proton beams and intensity modulated proton beams for intracranial lesions. Int J Radiat Oncol Biol Phys 2004;60(4):1314-1324.

Baumert BG, Villà S, Studer G, Mirimanoff RO, Davis JB, Landau K, Ducrey N, Arruga J, Lambin, P, Pica A. Early improvements in vision after fractionated stereotactic radiotherapy for primary optic nerve sheath meningioma. Radiother Oncol 2004;72:169-174

Stupp R and Baumert BG. Promises and controversies in the management of low-grade glioma. Editorial. Ann Oncol 2003;14: 1695-1696.

Baumert BG, Egli P, Studer S, Davis JB. Repositioning accuracy of fractionated stereotactic irradiation: Assessment of isocentre alignment for different dental fixations by using sequential CT scanning. Radiother Oncol 2004; In press.

Baumert BG, Infanger M, Reiner B, Davis JB. A novel technique using customized templates for the application of fractionated interstitial brachytherapy to the tumour bed in soft tissue sarcomas located in the extremities. Clinical Oncology (R Coll Radiol) 2004;16(7):457-460

Landert M, Baumert BG, Luetolf UM, Boesch MM, Landau K. Outcome of optic nerve sheath meningioma treated by fractionated stereotactic radiotherapy. Submitted for publication Neuroophthalmology 2004. 


\section{ACKNOWLEDGEMENT}

As everything else in life this thesis is not a solitary pursuit, but the result of a team effort and thus I would like to acknowledge those people who have been of constructive help. I remain grateful to those cited below and to all those not mentioned, it was not meant to be forgotten, but as it happens, it is almost impossible to catalogue all those to whom I am indebted to for helpfulness and willingness to listen and think through a problem. In the foreground, there is the research group of colleagues, to whom I would like to offer my sincere gratitude, without whose assistance, editing and commitment the publications would not have been possible in such a relatively short period of time.

In first place I would like to thank my promotor Philippe Lambin, who made this thesis possible with his continuous support scientifically and otherwise, encouragement and stimulating ideas (and impossible deadlines).

I want to give special thanks to Bernard Davis, for support, advice and discussions, for his support in fighting with linguistic edges, for making studies possible and for staying by my side even if at times I was unbearable. He somehow found the time for transforming my vastly detailed avalanche of words into a manageable text despite my protest and survived my thousands of continuous e-mails demanding attention. And last but not least, I also thank him for the remarkable job of reviewing nearly all manuscripts and detecting many details needing changing.

Further, I want to give my thanks to Ian Norton for his insisting argumentations and eager readiness in comparative physics research, Vesselin Miltchev and Peter Egli for generating data, Anthony Lomax for providing us with constructive arguments and criticisms during the development of the study and the manuscript, Klara Landau for multidisciplinary research and ideas, Frank Saran for offering his experience in gathering data at the Royal Marsden Hospital and guidance through the manuscript, Johannes Lutterbach and Salvador Villá for exchange of data and research ideas. Thank you to Cary Dehing for taking the time and her patience in explaining statistics to me that I could understand it.

For René Mirimanoff's valuable advice and general enthusiastic support expressed through his imperturbable and unchangeable belief in my seemingly endless energy, as he used to describe it.

I would like to take this opportunity to thank Michael Brada, as this was not possible until now. He has my esteem as my mentor and as a valuable teacher taking the time for listening and giving advice. My time at the Royal Marsden Hospital in London and Sutton, U.K., I remember as a time full of fruitful discussions often starting with different points of view and resulting in new ideas for future research. 
Thank you to my colleagues of the Dept. of Radiation-Oncology (MAASTRO) in Maastricht for support in solving clinical problems when I was not there, making it possible in giving me time for writing and research, especially Jos Jager for enduring me in our shared office during the last months when this booklet was being produced.

Thank you to my "neuro-"colleagues of the Academic Hospital Maastricht, especially Henk van Santbrink (of the Department of Neurosurgery), Albert Twijnstra (Neurology) and Jan Wilmink (Neuroradiology) for their patience and readiness for co-operation.

A special thanks to the working group of Stereotactic Radiotherapy of the MAASTRO Clinic for their encouraging enthusiasm and the continuous input of ideas and developmental work.

Thank you to my colleagues of the University Hospital Zurich, Switzerland, especially René Bernays from the Department of Neurosurgery and the working group for Stereotactic Radiotherapy of the Radiation - Oncology department (with special thanks to Emily Rabe and Martina Fuksa-Cabiallavetta), without whom the treatment and studies of all those patients would not have been possible; Hans Schuknecht from the Department of Neuroradiology, from whom I learnt most of my neuroradiologic anatomy. Many colleagues contributed, each in their own way, to my training in Radiation Oncology as well as to the development of my research; last not least Urs Martin Lütolf, Chairman of Radiation Oncology, who prepared the way for Stereotactic Radiotherapy in his clinic.

Many others contributed to the development of the research ideas through the sharing of thoughts and knowledge wherever the meeting place was: thanks to Harry Bartelink, Howard Thames, Jim Warrington, Ben Slotman, Eric Bouffet, Roger Stupp, Silvia Hofknecht, André Minken, Vincent Khoo, and many others too numerous to mention.

My parents, my father who I am sure would have supported me with all his means, if he would have known about it, my mother for her patience and for listening to me when I needed it and for turning my attention back to other needs and aspects of life in grounding me with humour.

My friends and colleagues Barbara Zwinggi and Susan Short for buoying me up when necessary. 
FELIX QUI POTUIT RERUM COGNOSCERE CAUSAS (VERGIL) 